\title{
Unfallgefährdung von Motorradfahrern
}

Reinhold Maier

Volker Schindler

Matthias Körner

Thomas Scholz

Martin Unger

Matthias Kühn 


\title{
Unfallgefährdung von Motorradfahrern
}

\author{
Univ.-Prof. Dr.-Ing. Reinhold Maier \\ Prof. Dr. rer. nat. Volker Schindler \\ Dipl.-Ing. Matthias Körner \\ Dipl.-Ing. Thomas Scholz \\ Dipl.-Ing. Martin Unger \\ Dr.-Ing. Matthias Kühn
}


Die Unfallforschung der Versicherer veröffent-

licht ihre Forschungsergebnisse in den Reihen:

FS - Fahrzeugsicherheit

VI - Verkehrsinfrastruktur

VV - Verkehrsverhalten / Verkehrspädagogik

Impressum:

Gesamtverband der Deutschen Versicherungswirtschaft e. V.

Unfallforschung der Versicherer

Wilhelmstraße 43 / 43G, 10117 Berlin

Postfach 0802 64, 10002 Berlin

E-Mail: unfallforschung@gdv.de

Internet: www.unfallforschung-der-versicherer.de

ISBN-Nr.: 978-3-939163-22-0

Redaktion: Dr.-Ing. Matthias Kühn

Gestaltung: Michaela Gaebel

Druckerei: GDV e. V.

Berlin, Januar 2009 


\section{Unfallgefährdung von Motorradfahrern}

bearbeitet durch

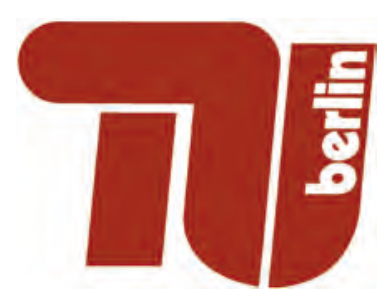

Technische Universität Berlin

Institut für Land- und Seeverkehr

Fachgebiet Kraftfahrzeuge

Prof. Dr. rer nat. Volker Schindler

Dipl.-Ing. Martin Unger

und

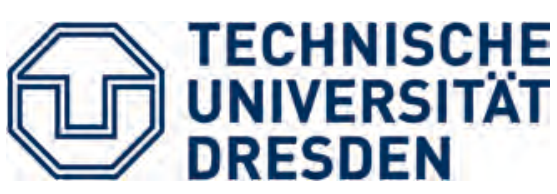

Technische Universität Dresden Fakultät Verkehrswissenschaften „Friedrich List“

Professur für Straßenverkehrstechnik mit Fachbereich Theorie der Verkehrsplanung

Univ.-Prof. Dr.-Ing. Reinhold Maier

Dipl.-Ing. Matthias Körner

Dipl.-Ing. Thomas Scholz

Bei der UDV betreut durch:

Dr.-Ing. Matthias Kühn 


\section{Inhalt}

Abkürzungsverzeichnis

$1 \quad$ Einleitung

1.1 Klassifizierung der motorisierten Zweiräder (PTW) nach

Zulassungsvorschriften

1.2 Klassifizierung der motorisierten Zweiräder (PTW) nach Art des Motorradsegments

1.3 Festlegung von Zielgruppen

$2 \quad$ Projektgliederung

2.1 Beschreibung des Projektablaufes

2.2 Verknüpfung der unterschiedlichen Datenbasen

$3 \quad$ Stand der Erkenntnisse - Literaturauswertung

3.1 Vorhandene Erkenntnisse aus dem Unfallgeschehen

3.1.1 Untersuchung des Unfallgeschehens

3.1.2 Untersuchung des Unfallgeschehens mit Einbezug von Straßendaten

3.2 Literaturstudie - Gefahren für den Motorradfahrer aus Sicht der Fahrzeugtechnik

$4 \quad$ Entwicklung des Unfallgeschehens

4.1 Generelle Entwicklungen

4.1.1 Fahrzeugbestand

4.1.2 Fahrleistungen von Kraftfahrzeugen 34

4.1.3 Anzahl und Schwere der Unfälle 36

4.1.4 Raten - Maß für die relative Unfallgefahr 38

4.2 Unfallgeschehen der Zielgruppen 40

$5 \quad$ Unfallgeschehen und Straßeneigenschaften 44

5.1 Methodik 44

5.1.1 Untersuchungsgebiet 44

5.1.2 Auswahl und Auswertung der Streckenabschnitte in Sachsen 46

5.1.3 Streckenbesichtigungen 48

5.1.3.1 Auswahl der Streckenabschnitte 48

5.1.3.2 Aufgenommene Daten 49

5.1.4 Datenauswertung der besichtigten Streckenabschnitte 49

5.2 Ergebnisse 51

5.2.1 Unfallgeschehen in Sachsen 51

5.2.2 Unfallgeschehen auf den ausgewählten Streckenabschnitten 53

5.2.3 Unfallgeschehen und Straßeneigenschaften auf den besichtigten Streckenabschnitten 
5.2.3.1 Außerorts 59

5.2.3.2 Innerorts 63

5.2.4 Detailanalyse typischer Unfallkonstellationen 65

$6 \quad$ Unfallgeschehen und Fahrzeugtechnik 69

6.1 Auswertung der Daten des Statistischen Bundesamtes 71

6.2 Auswertung der Daten des GDV 73

6.2.1 Verletzungsschwereverteilung $\quad 73$

6.2.2 Hauptanstoß $\quad 74$

6.2.3 Alkohol und überhöhte Geschwindigkeit 76

6.2.4 Motorradtyp 76

6.2.5 Leistungsgewicht 76

6.2.6 Unfallbetrachtung nach Wochentagen 82

6.2.7 Verkehrsdichte $\quad 82$

6.2.8 Reaktion vor einem Unfallereignis 84

6.2.9 Unfallablauf und Hauptverletzungsregion 85

6.2.10 Überflug im Fall einer Kollision 86

6.2.11 Anteil der Unfallverursacher 86

6.2.12 Zusammenfassung 89

6.3 Auswertung der Daten der Motorradumfrage 90

6.3.1 Altersverteilung 91

6.3.2 Verteilung der Motorradtypen in der Umfrage 92

6.3.3 Motivation zum Motorradfahren 92

6.3.4 Nutzungsverhalten 92

6.3.5 Selbstgeschätztes Fahrkönnen und Fahrstil 96

6.3.6 Straßennutzungsverhalten 98

6.3.7 Verkehrsverstöße 99

6.3.8 Tragen von Sicherheitskleidung 101

6.3.9 Unfallbetrachtung 103

$\begin{array}{ll}6.3 .10 & \text { Zusammenfassung } \\ & 105\end{array}$

$7 \quad$ Zusammenführung der Ergebnisse $\quad 107$

$\begin{array}{ll}7.1 & \text { Unfallkonstellation (1) }\end{array}$

7.2 Unfallkonstellation (2) 109

7.3 Unfallkonstellation (3) 111

7.4 Unfallkonstellation (4) 112

$\begin{array}{lll}7.5 & \text { Unfallkonstellation (5) } & 115\end{array}$

$\begin{array}{lll}7.6 & \text { Unfallkonstellation (6) } & 116\end{array}$

$\begin{array}{lll}7.7 & \text { Unfallkonstellation (7) } & 118\end{array}$

$\begin{array}{lll}7.8 & \text { Unfallkonstellation (8) } & 119\end{array}$

$\begin{array}{ll}7.9 & \text { Unfallkonstellation (9) } \\ 7.101\end{array}$

$\begin{array}{ll}7.10 & \text { Unfallkonstellation (10) }\end{array}$ 
8 Ableitung von Maßnahmen

123

8.1 Maßnahmen zur Unfallkonstellation

123

8.2 Technische Maßnahmen

8.2.1 Rückhaltesysteme

123

8.2.2 Fahrzeugstruktur

123

8.2.3 Begrenzung des Beschleunigungsvermögens 125

8.3 Verkehrserziehungsmaßnahmen $\quad 125$

8.3.1 Extrinsische Maßnahmen 125

8.3.2 Intrinsische Maßnahmen 126

8.4 Maßnahmen beim Unfallgegner 126

$\begin{array}{lll}9 & \text { Fazit und Ausblick } & 127\end{array}$

$\begin{array}{ll}10 & \text { Abbildungsverzeichnis }\end{array}$

11 Tabellenverzeichnis 133

12 Literaturverzeichnis 134

13 Anhang 136 


\section{Abkürzungsverzeichnis}

\begin{tabular}{|l|l|}
\hline AB-Unfall & Abbiegeunfall (Unfalltyp 2 nach [FGSV 2003]) \\
\hline ago & außerhalb geschlossener Ortschaften \\
\hline AIS & Abbreviated Injury Scale \\
\hline BASt & Bundesanstalt für Straßenwesen \\
\hline DIW & Deutsches Institut für Wirtschaftsforschung \\
\hline EK-Unfall & Einbiegen-/Kreuzen-Unfall (Unfalltyp 3 nach [FGSV 2003]) \\
\hline EW & Einwohner \\
\hline HBS & Handbuch für die Bemessung von Straßenverkehrsanlagen \\
\hline igo & innerhalb geschlossener Ortschaften \\
\hline KBA & Kraftfahrzeugbundesamt \\
\hline Krad & Kraftrad \\
\hline LV & Längsverkehr \\
\hline MAIS & Maximaler Wert der Abbriviated Injury Scale \\
\hline PTW & Powered-Two-Wheeler / motorisiertes Zweirad \\
\hline UD & Unfalldichte \\
\hline UH & Unfallhäufung \\
\hline$U(P)$ & Unfall mit Personenschaden \\
\hline$U(S)$ & Unfall mit Sachschaden \\
\hline$U(S S)$ & Unfall mit schwerwiegendem Sachschaden \\
\hline UKR & Unfallkostenrate \\
\hline VUA & Verkehrsunfallanzeige \\
\hline WU & mittlerer Unfallkostensatz \\
\hline Z 108 StVO & Zeichen 108 nach Straßenverkehrsordnung \\
\hline & \\
\hline &
\end{tabular}




\section{$1 \quad$ Einleitung}

Im Straßenverkehr sinkt die Zahl der tödlich verunglückten Unfallopfer seit Jahren stetig und hat im Jahr 2006 ein Minimum von 5.091 Verkehrstoten erreicht. Die Zahl der im Straßenverkehr getöteten Motorradfahrer ist jedoch nahezu konstant und schwankt ohne große Sprünge um 900. Im Jahr 2006 waren es 793 [Destatis 2007]. Damit stellen die Motorradfahrer ca. $16 \%$ der im Straßenverkehr getöteten Personen, machen bestandsbezogen aber nur einen Anteil von rund $8 \%$ [BMVBS 2006] aus und sind somit in der Unfallstatistik überrepräsentiert. Das Todesrisiko liegt dabei für den Aufsassen eines Motorrades 3 mal höher als für einen Pkw-Insassen [Koch 2006]. Betrachtet man das Risiko bei einem Motorradunfall getötet zu werden fahrleistungsabhängig, so liegt es sogar 12 mal höher als bei einem Pkw [Koch 2006].

Der positive Trend der Zahl der getöteten Pkw Insassen wird häufig mit den zahlreichen aktiven und passiven Sicherheitseinrichtungen am Fahrzeug in Verbindung gebracht. Moderne Pkws sind selbst in der kleinen Fahrzeugklasse schon mit $A B S$ ausgerüstet und verfügen wenigstens über zwei Airbags serienmäßig. Dagegen sind in den letzten Jahren die aktiven Sicherheits-einrichtungen an Motorrädern zwar auch durch immer häufiger verfügbares ABS verbessert worden, doch fehlt hier eine vorangeschrittene Marktdurchdringung und Fahrerakzeptanz. Im Bereich der passiven Sicherheit kann als ernst zunehmender Ansatz nur der BMW C1 betrachtet werden, der jedoch im Jahr 2003 nach 30.000 Einheiten eingestellt wurde.

Im Forschungsprojekt „Unfallgefährdung von Motorradfahrern" untersucht das Fachgebiet Kraftfahrzeuge der TU Berlin mit der Professur Straßenverkehrstechnik der TU Dresden interdisziplinär diejenigen Einflussfaktoren, die eine hohe Korrelation zu Verletzungsmustern der verunglückten Motorradfahrer aufweisen.

Aus diesen Einflussfaktoren werden im Anschluss sowohl fahrzeug- als auch verkehrstechnische Maßnahmen aufgestellt, die es ermöglichen, die Motorradsicherheit besser an die positive Entwicklung der übrigen Verkehrs- teilnehmer anzukoppeln. Dabei sollen vorrangig gerade die Eigenschaften ermittelt werden, welche durch eine Wechselwirkung von Verkehrstechnik und Fahrzeugtechnik zum Unfall und dementsprechend zur Verletzung oder zum Tode führen.

\subsection{Klassifizierung der motorisierten Zweiräder (PTW) nach Zulassungs vorschriften}

Bei der Einteilung von motorisierten Zweiräder (PTW) wird hinsichtlich der Vorschriften über das Zulassungsverfahren, ihrer Bauart sowie bestimmter Leistungskennwerte ihres Motors unterschieden.

Kraftfahrzeuge, die im Straßenverkehr geführt werden, benötigen entsprechend $\S 18$ StVZO eine Erteilung einer Betriebserlaubnis oder eine EG-Typgenehmigung sowie eine Zuteilung eines amtlichen Kennzeichens für Kraftfahrzeuge von der Verwaltungsbehörde (Zulassungsbehörde). Ausgenommen von der Vorschrift zum Zulassungsverfahren sind nach $\S 18$ Abs. 2 StVZO u.a. zweirädrige Kleinkrafträder, Fahrräder mit Hilfsmotoren und Leichtkrafträder. Ein amtliches Kennzeichen müssen Leichtkrafträder nach $\S 18$ Abs. 4StVZO, wenn sie beim Verkehr auf öffentlichen Straßen betrieben werden sollen, dennoch führen (Versicherungskennzeichen). Weitere Abgrenzungen werden mit fahrzeugspezifischen Kennwerten vorgenommen. Dafür sind Grenzwerte für Hubraum, Nennleistung und maximale Geschwindigkeit von Bedeutung. Die motorisierten Zweiräder werden in fünf verschiedene Klassen aufgeschlüsselt, die häufig zu zwei Gruppen zusammengefasst werden. Einen Überblick der einzelnen Klassen und der aktuellen Gruppenbildung zeigt Tabelle 1-1.

Bei den Leichtkrafträdern müssen beide Bedingungen aus den Motorangaben erfüllt sein. Motorräder werden dann als Kraftrad oder Kraftroller bezeichnet, wenn mindestens ein Grenzwert (Hubraum oder Nennleistung) überschritten ist. 


\begin{tabular}{|c|c|c|c|c|c|c|}
\hline \multirow{2}{*}{$\begin{array}{l}\text { Schlüssel- } \\
\text { nummer }\end{array}$} & \multirow[t]{2}{*}{ Bezeichnung } & \multicolumn{2}{|c|}{ Motorangaben } & maximale & \multirow{2}{*}{\multicolumn{2}{|c|}{$\begin{array}{c}\text { Bezeichnung } \\
\text { Destatis }\end{array}$}} \\
\hline & & Hubraum & Nennleistung & Geschw. & & \\
\hline 01 & Kleinkraftrad & $\leq 50 \mathrm{~cm}^{3}$ & k. A. & $\leq 50 \mathrm{~km} / \mathrm{h}$ & \multirow{2}{*}{$\begin{array}{c}\text { Mofa/Moped } \\
\text { (mit Versicherungs- } \\
\text { kennzeichen }\end{array}$} & \multirow{5}{*}{ 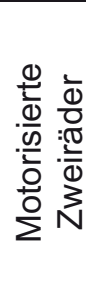 } \\
\hline 02 & Mofa 25 & $\leq 50 \mathrm{~cm}^{3}$ & k. A. & $\leq 25 \mathrm{~km} / \mathrm{h}$ & & \\
\hline 11 & Kraftrad & $>125 \mathrm{~cm}^{3}$ & $>11 \mathrm{~kW}$ & k. A. & \multirow{3}{*}{$\begin{array}{c}\text { Motorrad } \\
\text { (mit amtlichen } \\
\text { Kennzeichen) }\end{array}$} & \\
\hline 12 & Leichtkraftrad & $51-125 \mathrm{~cm}^{3}$ & $\leq 11 \mathrm{~kW}$ & k. A. & & \\
\hline 15 & Kraftroller & $>125 \mathrm{~cm}^{3}$ & $>11 \mathrm{~kW}$ & k. A. & & \\
\hline
\end{tabular}

Tabelle 1-1: Einteilung der motorisierten Zweiräder nach StVZO und Statistischem Bundesamt (Destatis)

Die Krafträder werden hinsichtlich ihrer Bauart noch einmal in Kraftrad und Kraftroller unterschieden. Diese Trennung fehlt weiterführend bei motorisierten Zweirädern mit geringerer Nennleistung und Hubraum. Somit sind in den Klassen Leichtkraftrad und Kleinkraft auch Roller enthalten.

Für die fünf Motorradklassen sind in der amtlichen Statistik Schlüsselnummern vergeben. Diese Codierung ist vom Unfall aufnehmenden Polizisten zu beachten und als Art der Verkehrsbeteiligung in die Unfallanzeige einzutragen. Somit ist eine feingliedrige Auswertung des Unfallgeschehens von motorisierten Zweiradfahrern gegeben.

Orientierend an der EG-Betriebserlaubnisrichtlinie für zwei- oder dreirädrige Kraftfahrzeuge wurden beim Kraftfahrzeugbundesamt (KBA) Flensburg-Dresden motorisierten Zweiräder in sechs Klassen unterschieden. Für die Krafträder und Kraftroller gab es noch das Unterscheidungsmerkmal „Beiwagen“ (mit/ohne). In der Beschreibung der Fahrzeugart (Stand: Juni 2005) wird vermerkt, dass die Ausweisung von Rollern, Krafträder mit Beiwagen sowie Moped aufgegeben worden sind. Weiterhin gelten Kleinkrafträder neueren Rechts nur noch solche mit einer Höchstgeschwindigkeit bis $45 \mathrm{~km} / \mathrm{h}$.

Mit der Umstellung der Verschlüsselung zum 1. Juni 2000 werden alle neu in den Verkehr kommenden motorisierten Zweiräder eine neue Schlüsselnummer erhalten. Somit wird beim KBA lediglich nach den zwei Gruppen Kleinkraftrad/Mofa (Fahrzeugart Nr. 24) und Leichtkraft$\mathrm{rad} /$ Kraftrad (Nr. 25) differenziert.
Für die Unterscheidung der Kraftfahrzeuge werden bei Versicherungen Gruppen hinsichtlich Fahrzeugart und Wagnisstärke (z. B. Hubraum, Motorleistung) gebildet. Jeder Gruppe einer Fahrzeugart wird eine Hauptwagniskennziffer zugeordnet. Die Krafträder werden in 10 verschiedene Versicherungsrisikogruppen unterteilt, wobei in einer Gruppe die drei- und vierrädrigen Trikes und Quads enthalten sind und in einer zweiten Gruppe die maschinell angetriebenen Krankenfahrstühle mit Versicherungskennzeichen, die in der Untersuchung nicht näher betrachtet werden. Von den verbliebenen acht Gruppen sind allein sechs Gruppen für die Leichtkrafträder sowie jeweils eine Gruppe für die Krafträder/Kraft und die Kleinkrafträder/Mofas vorgesehen.

Einen Vergleich der beschriebenen Einteilungen zeigt Tabelle 1-2. Eine Abgrenzung aller Rollerfahrzeuge ist aus den Gruppierungen nur dann möglich, wenn die Fahrzeuge vor dem 1. Juni 2000 eine Typzulassung erhalten haben.

\subsection{Klassifizierung der motorisierten Zweiräder (PTW) nach Art des Motorradsegments}

Neben den zulassungsbezogenen Klassifizierungen können Motorräder gemäß ihrer Marktpositionierung eingeteilt werden. An dieser Stelle wird auf die Kategorien nach [Sporner 2006] zurückgegriffen.

Sportmaschinen (Abbildung 1-1) zeichnen sich durch eine Vollverkleidung und weit heruntergezogene Lenkerstummel aus. Für eine größere Schräglagenfreiheit sind Aus- 


\begin{tabular}{|c|c|c|c|c|c|c|c|}
\hline \multirow[t]{3}{*}{ Bezeichnung } & \multicolumn{2}{|c|}{ Motorangaben } & \multirow{3}{*}{$\begin{array}{l}\text { Mindest- } \\
\text { alter des } \\
\text { Halters }\end{array}$} & \multicolumn{4}{|c|}{ Nummerierung } \\
\hline & \multirow[t]{2}{*}{ Hubraum } & \multirow{2}{*}{$\begin{array}{l}\text { Nenn- } \\
\text { leistung }\end{array}$} & & \multirow[t]{2}{*}{ Destatis } & \multicolumn{2}{|c|}{ KBA } & \multirow[t]{2}{*}{ Versicherung } \\
\hline & & & & & alt & neu & \\
\hline Kleinkraftrad & $\leq 50 \mathrm{~cm}^{3}$ & k. A. & k. A. & \multirow{2}{*}{01} & M2 & \multirow{3}{*}{24} & \multirow{3}{*}{005} \\
\hline Kleinkraftroller & $\leq 50 \mathrm{~cm}^{3}$ & k. A. & k. A. & & M3 & & \\
\hline Mofa 25 & $\leq 50 \mathrm{~cm}^{3}$ & k. A. & k. A. & 02 & M1 & & \\
\hline \multirow[t]{3}{*}{ Leichtkraftrad } & $\leq 80 \mathrm{~cm}^{3}$ & $\leq 11 \mathrm{~kW}$ & 16 Jahre & \multirow{6}{*}{12} & \multirow{6}{*}{ M4/M5 } & \multirow{8}{*}{25} & 022 \\
\hline & $\leq 125 \mathrm{~cm}^{3}$ & $\leq 11 \mathrm{~kW}$ & 16 Jahre & & & & 026 \\
\hline & $\leq 125 \mathrm{~cm}^{3}$ & $\leq 11 \mathrm{~kW}$ & 18 Jahre & & & & 028 \\
\hline \multirow[t]{3}{*}{ Leichtkraftroller } & $\leq 80 \mathrm{~cm}^{3}$ & $\leq 11 \mathrm{~kW}$ & 16 Jahre & & & & 012 \\
\hline & $\leq 125 \mathrm{~cm}^{3}$ & $\leq 11 \mathrm{~kW}$ & 16 Jahre & & & & 016 \\
\hline & $\leq 125 \mathrm{~cm}^{3}$ & $\leq 11 \mathrm{~kW}$ & 18 Jahre & & & & 018 \\
\hline Kraftrad & $>125 \mathrm{~cm}^{3}$ & $>11 \mathrm{~kW}$ & k. A. & 11 & $09 / 19$ & & \multirow{2}{*}{003} \\
\hline Kraftroller & $>125 \mathrm{~cm}^{3}$ & $>11 \mathrm{~kW}$ & k. A. & 15 & $39 / 49$ & & \\
\hline
\end{tabular}

Tabelle 1-2: Vergleich der Einteilungen von motorisierten Zweirädern

puff und Fußrasten hoch platziert. Der Fahrer sitzt mit einem engen Kniewinkel tief nach unten gebeugt und eng am Tank anliegend um bei hohen Geschwindigkeiten dem Fahrtwind einen geringen Widerstand zu bieten. Die Tankform ist in Richtung Becken des Fahrers sehr weit hochgezogen.

Bei Naked Bikes (Abbildung 1-2) wird zumeist ein durchgehender Lenker verwendet, der durch eine relativ aufrechte Sitzposition ein entspanntes Fahren ermöglicht. Auch der Kniewinkel ist größer als bei den Sportmaschinen. Durch die fehlende Verkleidung gibt es keinen Windschutz für den Fahrer. Den Stylingtrends entsprechend sind Naked Bikes oft Sportmaschinen, bei denen nur die Verkleidung durch den Hersteller oder Kunden entfernt wurde.

Die Bezeichnung Chopper (Abbildung 1-3) stammt aus der Zeit, als zum Tuning der Motorräder alles „unnötige“ Gewicht entfernt wurde (engl. „to chop“ - abhacken, abschneiden). Zu den heutigen Motorrädern würde der Name Cruiser besser passen, da Chopper im allgemeinen schwerer als Sportmaschinen oder Naked Bikes sind. Auch weisen Chopper oft, gegen die ursprüngliche Bezeichnung, verspielte Verzierungen auf. Chopper zeich- nen sich durch einen sehr niedrigen Schwerpunkt aus, der aus einer niedrigen Sitzposition und einer geringen Bodenfreiheit resultiert. Der aktuelle Trend führt zu immer größeren Gesamt- und Einzelhubräumen und erreicht bei der Yamaha XV 1900 Midnight Star mit 1.854 $\mathrm{cm}^{3}$ für einen Zweizylinder ein momentanes Maximum.

Oldtimer (Abbildung 1-4) sind klassische Motorräder älter als 30 Jahre. In der vorliegenden Untersuchung spielen sie praktisch keine Rolle.

Enduros (Abbildung 1-5) sind speziell für den Offroadeinsatz entwickelte Zweiräder. Sie haben Reifen mit ausgeprägten Stollen und lange Federwege. Die Sitzposition ist aufrecht und nah am Lenker. Der Übergang von Sitzbank zum Tank ist fließend. Um das Gewicht niedrig zu halten sind meist Einzylindermotoren verbaut.

Roller (Abbildung 1-6) bieten eine komfortablere Sitzposition, da sie kein Oberrohr besitzen. Der Motor sitzt direkt an der Schwinge und zumindest die Beine sind vor Witterungseinflüssen geschützt. Primär für den urbanen Gebrauch eingesetzt haben sie in der Regel eine geringe Leistung und kleine Raddurchmesser. 
Tourer (Abbildung 1-7) sind schwere, vollverkleidete und komfortable Motorräder, die speziell für lange Einzelstrecken, alleine oder zu zweit, ausgerüstet sind. Für Gepäck sind die Koffer zumeist in die Verkleidung integriert. Ausstattungsmerkmale können Sitzheizung, Rückwärts-

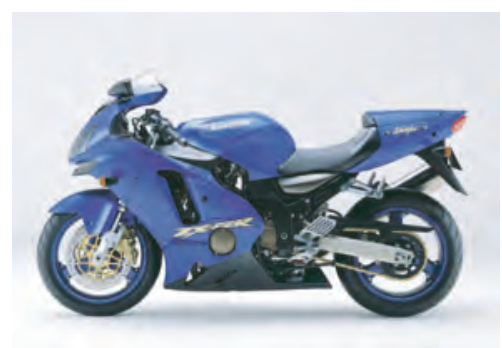

Abbildung 1-1: Sportmaschine

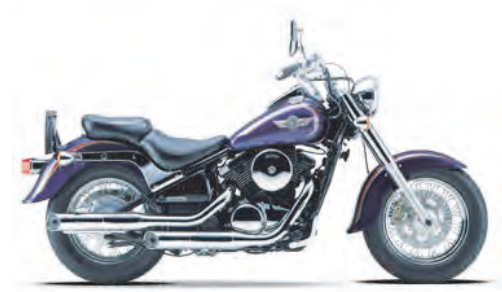

Abbildung 1-3: Chopper

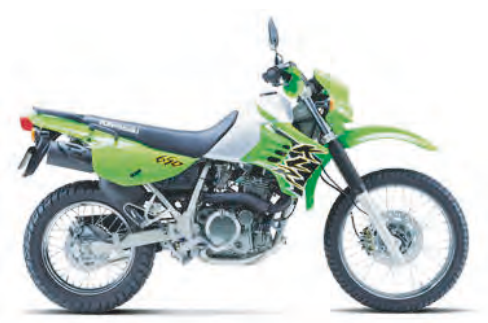

Abbildung 1-5: Enduro

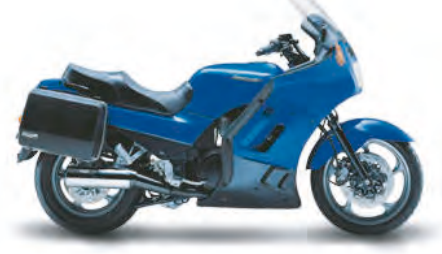

Abbildung 1-7: Tourer gang, CD-Wechsler, elektrisches Windschild und Gegensprechanlage sein, um nur einige zu nennen.

Mofas (Abbildung 1-8) erinnern an motorisierte Fahrräder und spielen in dieser Untersuchung keine Rolle.

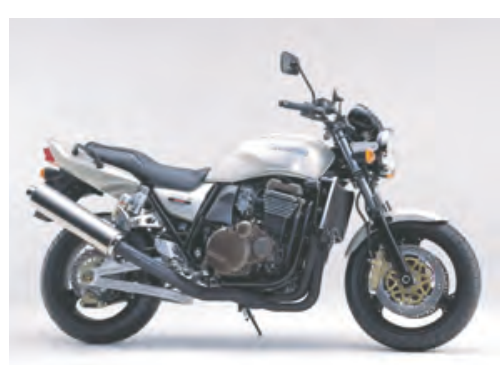

Abbildung 1-2: Naked Bike

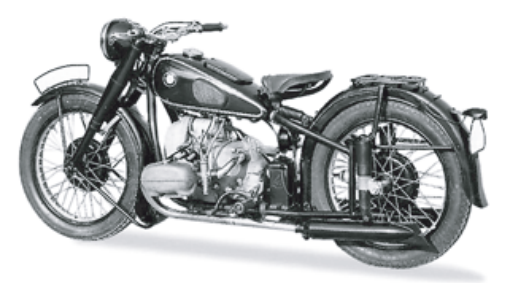

Abbildung 1-4: Oldtimer

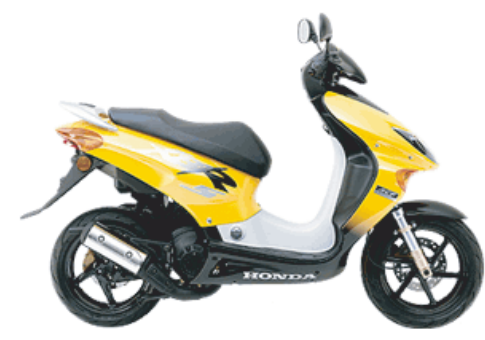

Abbildung 1-6: Roller

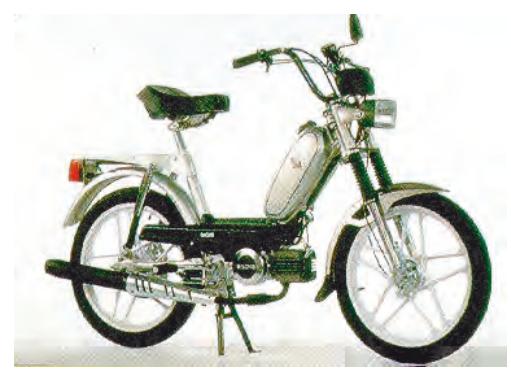

Abbildung 1-8: Mofa 


\subsection{Festlegung von Zielgruppen}

Ausgangspunkt für die Bildung von Zielgruppen war die Überlegung, dass sich bei Motorradfahrern die Motivation und daraus folgende Verhaltensweisen, angefangen von der Auswahl des Fahrzeugs bis zu den häufigen Fahrtzeiten unmittelbar auf die Unfallgefahr auswirken können. Sie müssten daher gezielt beeinflusst werden, wenn sich diese Unterschiede nachweisen lassen. Beispielsweise können Wiedereinsteiger, die zwar einen Motorradführerschein besitzen, seit längerer Zeit diesen aber nicht mehr benutzt haben, eine besondere Problematik darstellen. Im Hinblick auf die Kommunikation mit der Fachöffentlichkeit und der allgemeinen interessierten Öffentlichkeit sollte eine Sicherheitsuntersuchung von motorisierten Zweiradfahrern sowohl für die Verkehrsinfrastruktur als auch für die Fahrzeugausstattung auf solche Zielgruppen fokussiert werden. Dafür werden folgende sieben Zielgruppen vorgeschlagen, wobei die Bezeichnungen lediglich eine generelle Adressierung und nicht eine umfassende Charakterisierung darstellen:

- Jugendliche: Dies sind Verkehrsteilnehmer unter 18 Jahre, die mit Fahrzeugen von maximal $125 \mathrm{~cm}^{3} \mathrm{Hub}$ raum, i. d. R. kleine Rollerfahrzeuge, unterwegs sind. Die Unfälle geschehen hauptsächlich innerorts, aber ansonsten bei allen Witterungsbedingungen und zu allen Zeiten (ggf. können bestimmte Zeiten, z. B. wegen Ferien, ausgeschlossen werden).

- Fahranfänger: Dies betrifft zum einen diejenigen, die mit 18 Jahren einen Motorradführerschein erwerben und dann ein Motorrad über $125 \mathrm{~cm}^{3}$ fahren, und zum anderen die Gruppe, welche ab 21 Jahren die nächstgrößere Klasse von Motorrädern erwerben und fahren. Das Alter der Fahrerlaubnis soll in beiden Fällen maximal 24 Monate betragen.

- Schlechtwetterunfälle: In diesem Fall handelt es sich um eine spezielle Unfallgruppe. Es werden die Unfälle mit motorisierten Zweirädern betrachtet, die sich in den Wintermonaten November bis März ereignet haben oder bei denen in der Unfallanzeige als Fahrbahnzustand Winterglätte oder Nässe ausgewiesen ist.
- Frauen: Der Gruppe werden alle weiblichen Fahrzeugführer der motorisierten Zweiräder als Unfallbeteiligte zugeordnet. In dieser Gruppe sind weibliche Motorradfahrer ab 21 Jahren, die Fahrzeuge mit mehr als $125 \mathrm{~cm}^{3}$ bewegen und auf allen Straßenarten (außer Autobahn), meist zur Tageszeit unterwegs sind.

- Rollerfahrer (Pragmatiker): Dies sind Verkehrsteilnehmer mit einem Kraftroller oder Roller von einem Hubraum größer als $125 \mathrm{~cm}^{3}$ bzw. bis $125 \mathrm{~cm}^{3}$.

- Wiedereinsteiger: Auswertungen haben gezeigt, dass sich diese Gruppe nicht ausreichend genau aus den Angaben der Unfallaufzeichnungen abgrenzen lässt bzw. Kriterien zu deren Abgrenzung fehlen. An dieser Stelle erfolgt dennoch eine eingrenzende Beschreibung der Zielgruppe, da die Gruppe als nicht unbedeutend für das Unfallgeschehen angesehen wird. Ist - zumindest annähernd - eine Eingrenzung bei den späteren Auswertungen möglich, sollte davon Gebrauch gemacht werden. Die Altersgruppe liegt vermutlich oberhalb der 35 Jahre. Sie ist männlich und bewegt schwere und starke Fahrzeuge $\left(a b 250 \mathrm{~cm}^{3}\right)$. Die Zeiten liegen im Sommer und die Witterung ist eher trocken. Zu prüfen wäre, inwieweit Nässe oder Nacht besondere Gefahren für diese Gruppen darstellen, und ob sich die Fahrbewegungen hauptsächlich am Wochenende konzentrieren.

- Fahrer ab 55: Es handelt sich um motorisierte Zweiradfahrer mit einem Lebensalter von 55 Jahren und mehr. Diese Fahrer bewegen alle motorisierten Zweiradfahrzeuge, auf allen Straßen (außer BAB) und zu allen Zeiten (meist bei Helligkeit).

In den EUSka-Daten, welche den Auswertungen in Sachsen zugrunde liegen, können die beschriebenen Gruppen mit Ausnahme der Wiedereinsteiger durch die Kriterien gemäß Tabelle 1-3 bestimmt werden:

Alle übrigen Abgrenzungskriterien wie Unfallzeit oder Unfallort - wenn zum Teil auch für die nähere Beschreibung der Gruppe gebraucht - werden nicht verwendet, sondern dienen - falls sinnvoll - der möglichen Unter- 
teilung der Zielgruppe. Die Zielgruppen sind nicht trennscharf, $d$. h., bestimmte Beteiligte können in mehreren Gruppen vorkommen. Sie ergänzen sich auch nicht zu $100 \%$ des Unfallgeschehens und dienen ausschließlich dazu, in Ergänzung der generellen Aussagen mögliche Auffälligkeiten bei solchen Zielgruppen darzustellen.

Diese Gruppen wurden für die weitere Untersuchung in folgenden Überlegungen berücksichtigt:

Bei der statistischen Auswertung wird geprüft, welchen Beitrag die Gruppen in Sachsen stellen. Zur Ergänzung dieser Information werden Abfragen (Sonderauswertungen) beim Statistischen Bundesamt durchgeführt, um für das gesamte Bundesgebiet Aussagen zumindest für die wichtigen Zielgruppen treffen zu können. Durch solche Auswertungen können bspw. Erwartungswerte für die Verteilung auf die Ortslage, die Lichtverhältnisse und den Straßenzustand bestimmt werden. Ähnliches

\begin{tabular}{|l|l|}
\hline Zielgruppe & Kriterium zur Abgrenzung \\
\hline Jugendliche & Fahreralter unter 18 Jahre \\
\hline Fahranfänger & $\begin{array}{l}\text { Führerscheinalter maximal } \\
\text { 2 Jahre }\end{array}$ \\
\hline Schlechtwetterunfälle & $\begin{array}{l}\text { Unfälle bei nasser oder } \\
\text { winterglatter Fahrbahn } \\
\text { oder in den Wintermona- } \\
\text { ten November bis März }\end{array}$ \\
\hline Frauen & Fahrergeschlecht weiblich \\
\hline Rollerfahrer & $\begin{array}{l}\text { Fahrzeugbeteiligung Schlüs- } \\
\text { selnummerr 15 (Kraftroller)* }\end{array}$ \\
\hline Wiedereinsteiger & $\begin{array}{l}\text { nicht definiert (Eingrenzung } \\
\text { über Fahreralter möglich) }\end{array}$ \\
\hline Fahrer ab 55 & $\begin{array}{l}\text { Fahreralter mindestens } \\
\text { 55 Jahre }\end{array}$ \\
\hline
\end{tabular}

Tabelle 1-3: Festlegung der Kriterien für die Bestimmung der Zielgruppen in EUSka gilt für Abfragen bzw. Auswertungen bei den Schäden der Versicherer.

Für die näher untersuchten Regionen in Sachsen sollen die Charakteristiken (z. B. Unfalltyp) dieser Gruppen herausgearbeitet werden, um dadurch mögliche Auffälligkeiten zu erkennen. Außerdem wird geprüft, ob sich von der Fahrzeugseite für diese Gruppen eine spezifische Beschreibung findet.

Weiterhin werden bestimmte Unfallkonstellationen, die an eine spezielle Charakteristik der Örtlichkeit gebunden sind (z. B. Unfälle in Kurven), daraufhin ausgewertet, welche Gruppen sich in bestimmten Konstellationen wiederfinden. Dies wird verglichen mit dem allgemeinen Auftreten dieser Gruppen. Je nach den Ergebnissen wurden die definierten Gruppen noch einmal angepasst, z. B. aufgrund zu geringer Besetzung der Gruppen (z. B. Fahrer ab 55, Frauen). 


\section{Projektgliederung}

\subsection{Beschreibung des Projektablaufes}

In den vergangenen Jahren hat sich gezeigt, dass die kontinuierlich positive Entwicklung der Verkehrssicherheit im Straßenverkehr nicht bei den Motorradfahrern widerspiegelt. In einem ersten Schritt sollen aus der Literatur die bereits vorliegenden Erkenntnisse zu den Gefahren für die Motorradfahrer aus der Sicht der Straße und der Fahrzeugtechnik herausgearbeitet werden. Diese sind durch Aussagen des aktuellen Unfallgeschehens zu festigen oder zu erweitern. Dabei soll die Sicherheitslage auf Basis der amtlichen Statistik neben den Unfällen mit Beteiligung motorisierter Zweiräder vergleichend auch für alle Unfälle umfassend beschrieben werden. Ergänzend zur Statistik sind Auswertungen mit der Unfalldatenbank UDB-VTIV vorzunehmen. In dieser Datenbank sind zusätzlich medizinische, fahrzeugtechnische und fahrerbezogene Aussagen zum speziellen Unfall enthalten, die wichtige Zusammenhänge aus der Entstehung des
Unfalls und seinen Folgen ableiten lassen, z. B. typische Verletzungsmuster und Fahrzeugbeschädigungen.

Einen weiteren Arbeitsschritt stellen Messungen und Befragungen zum Fahrer- und Fahrverhalten dar. Aufgrund der Ergebnisse können Auffälligkeiten bei bestimmten Personengruppen, Fahrzeugkategorien oder Fahrzeugeigenschaften festgestellt werden.

Auf Seiten der Verkehrstechnik erfolgt eine ortsbezogene Unfalldatenauswertung. Dafür werden im Untersuchungsgebiet Sachsen Bereiche mit hoher Unfalldichte in Bezug auf Unfälle mit motorisierten Zweirädern bestimmt. Für diese sind Streckenbesichtigungen mit Aufnahme der Straßendaten (Trassierung, Zustand, Ausstattung, etc.) vorgesehen, um anschließend Zusammenhänge zwischen Straßeneigenschaften und Unfallgeschehen zu untersuchen. Zu Vergleichszwecken werden Streckenabschnitte mit geringer Unfalldichte festgelegt

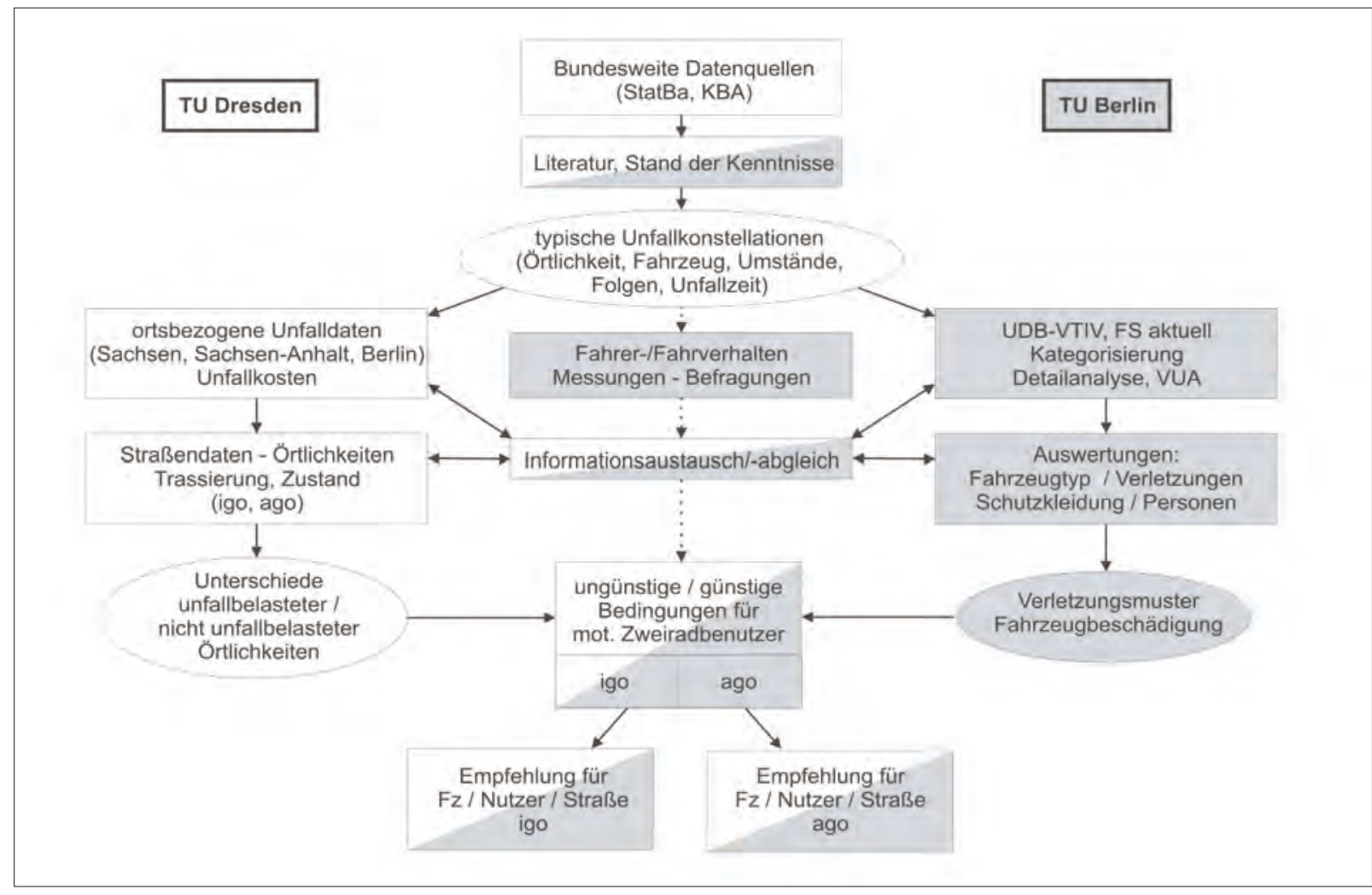

Abbildung 2-1: Struktur des Projektes 
und auch deren Straßeneigenschaften erfasst. Dies ermöglicht einen paarweisen Vergleich von unfallauffälligen und unfallfreien bzw. weniger unfallauffälligen Strecken, aus welchem straßenseitige, unfallbegünstigend wirkende Einflussfaktoren ermittelt werden können.

Die Ergebnisse der verschiedenen Auswertungen werden schließlich über die Charakteristik der Örtlichkeit sowie weiterer, in beiden Datenbasen vorkommender Merkmale (z. B. Unfalltyp) zusammengeführt, um daraus Erkenntnisse für die Günstigkeit/Ungünstigkeit von Bedingungen für motorisierte Zweiradbenutzer abzuleiten. Das Resultat sind nach Straßenlage (igo/ago) differenzierte Maßnahmenempfehlungen für Fahrzeug, Nutzer und Straße.

Abbildung 2-1 fasst den Projektablauf sowie die Aufteilung der Bearbeitungsschritte zwischen den beiden Projektbearbeitern TU Berlin und TU Dresden zusammen.

Im Detail sind folgende Arbeitsschritte vorgesehen:

\section{- Meilenstein 1:}

Nationale/internationale Literaturanalyse zu den Gefahren für den Motorradfahrer aus Sicht der Straße (TU Dresden) und der Fahrzeugtechnik (TU Berlin)

\section{- Meilenstein 2:}

umfassende Beschreibung der Sicherheitslage, vorrangig für die Unfälle mit Beteiligung motorisierter Zweiräder, vergleichend aber auch für alle Unfälle, auf Basis der amtlichen Statistik des Unfallgeschehens

\section{- Meilenstein 3:}

- ortsbezogene Detailanalyse von Motorradunfällen in Sachsen aus 3 Jahren, besondere Beachtung von Ortslage (innerorts/außerorts), Eigenschaften der Örtlichkeiten (z. B. Knotenpunktform, Längsneigung, etc.), Unfallablauf, Unfallbeteiligte, Lichtverhältnisse, Straßenzustand, Angabe zu den Personen (Alter, Fahrerfahrung)

- Abgleich mit den Erkenntnissen aus der Unfalldatenbank (UBD-VTIV) und FS aktuell. Charakterisierung des Unfalls: Anzahl der Beteiligten und Art der Verkehrsteilnahme, Fremd-/Eigenverschulden

- Ermittlung der Eigenschaften der am Unfall beteiligten Motorräder, die für Entstehen oder Verlauf relevant sein könnten wie Art des Motorrades (Lei- stung, Radführung, Bremse (ABS ja/nein, ABS-Typ)), Radgröße, Sitzposition (Haltung, Art des Lenkers etc.), Schutzausrüstung bei Fahrer und Mitfahrer (Schutzkleidung)

\section{- Meilenstein 4:}

Dokumentation und Untersuchung der Eigenschaften an Stellen, die in der örtlichen Unfalluntersuchung als besonders unfallträchtig bzw. unfallfrei erkannt wurden (Oberfläche, Trassierung, Seitenräume bei unterschiedlichen Witterungsbedingungen) Es soll ein paarweiser Vergleich der Charakteristik bei unfallbelasteten und bei geringen unfallbelasteten Örtlichkeiten durchgeführt werden. Bestimmung der Faktoren, die den Unfall begünstigt haben oder dessen Vermeidung verhinderten, sowie Bestimmung der Korrelation zwischen einzelner Fahrzeugeigenschaften und Verletzungsmuster der Motorradfahrer. Befragung zum Fahrer-/Fahrverhalten.

\section{- Meilenstein 5:}

Abgleich des Unfallgeschehens motorisierter Zweiradfahrer mit den übrigen Verkehrsteilnehmern (Unfallhäufung, Ähnlichkeit), Bewertung von Maßnahmen für den Kfz-Verkehr bezogen auf die Gefährdung von Motorradfahrern und besondere Maßnahmen für Motorradfahrer mit Rückwirkung auf den übrigen Kfz-Verkehr. Aufstellen eines Kataloges möglicher technischer Maßnahmen am Fahrzeug zur Reduzierung der unfalleinleitenden und verletzungsbestimmenden Fahrzeugeigenschaften.

\section{- Meilenstein 6:}

Zusammenführung der Ergebnisse mit den Erkenntnissen aus der Fahrzeug- und Straßenverkehrstechnik. Mögliche Rolle neuartiger Informationssysteme zwischen den Fahrzeugen sowie zwischen Motorrad und Straße. Mögliche Maßnahmen zur Verbesserung der Führerscheinausbildung. Technische Möglichkeiten zur Unterstützung des Lernprozesses beim „Wiedereinstieg" nach längerer Unterbrechung der Fahrpraxis oder nach Ende der Winterunterbrechung.

Die einzelnen Meilensteine sind zeitlich nicht streng voneinander getrennt, sondern greifen ineinander. So wurde z. B. die Motorradfahrerbefragung aus Meilenstein 4 vorgezogen und aufgrund des großen Umfangs bereits am Anfang des Projektes durchgeführt. 


\subsection{Verknüpfung der unterschiedlichen Datenbasen}

Gemäß dem Projektverlauf erarbeiten die beiden Projektpartner ihre Arbeitspakete losgelöst voneinander. Um interdisziplinär einen gemeinsamen Lösungsansatz zum Senken der Verunglücktenzahlen der Motorradfahrer aufzustellen, ist es jedoch notwendig, dass die einzeln herausgearbeiteten Ergebnisse trotz unterschiedlicher Datenbasen verknüpfbar gemacht werden.

Abbildung 2-2 zeigt die Systematik, auf der die Verbindung der Unfalldatengrundlagen von TU Dresden und TU Berlin aufbauen.
Die TU Dresden betrachtet die Unfälle von der Seite des Straßenraums mit dem Wissen der Ortslage und der Charakteristik der Unfallstelle. Die TU Berlin betrachtet die technischen Eigenschaften des Motorrades und die personenbezogenen Daten des Aufsassen, um dann über die Ortslage und Charakteristik der Unfallstelle eine Aussage über die Verletzungsmuster der Personen in Abhängigkeit der technischen Eigenschaften des Motorrades und des Verkehrsraumes treffen zu können. Weitere Unterteilungen, z. B. nach Unfalltyp und Unfallart, sind denkbar und inhaltlich sinnvoll. Zu berücksichtigen ist hierbei, dass die begrenzte Zahl an Einzelfällen die Summenhäufigkeit pro Einteilungseinheit für eine statistische Aussagefähigkeit gering werden lässt.
Art des Unfalls in Abhängigkeit von

- Anzahl und Schwere der Verunglückten

- Straßenzustand

- Lichtverhältnisse

- Lichtsignalanlage

- Aufprall auf Hindernis neben der Fahrbahn

- Hauptverursacher (Zweirad/Gegner)

- Art der Beteiligung des Unfallgegners

- Sicherheitsrelevante Zielgruppen

- Unfallzeitpunkt (Uhrzeit, Wochentag, Monat, Temperatur)

- Unfalltyp (einstellig)

- Unfallart
Ausmaß der Verletzung in Abhängigkeit von

- Art des Motorrades

- Hubraums

- Leistung

- Masse der Fahrzeuge

- Alter des Fahrzeuges

- Alter des Fahrers

- Geschlecht des Fahrers

- Alter des Führerscheins

- Unfalltyp

- Unfallart

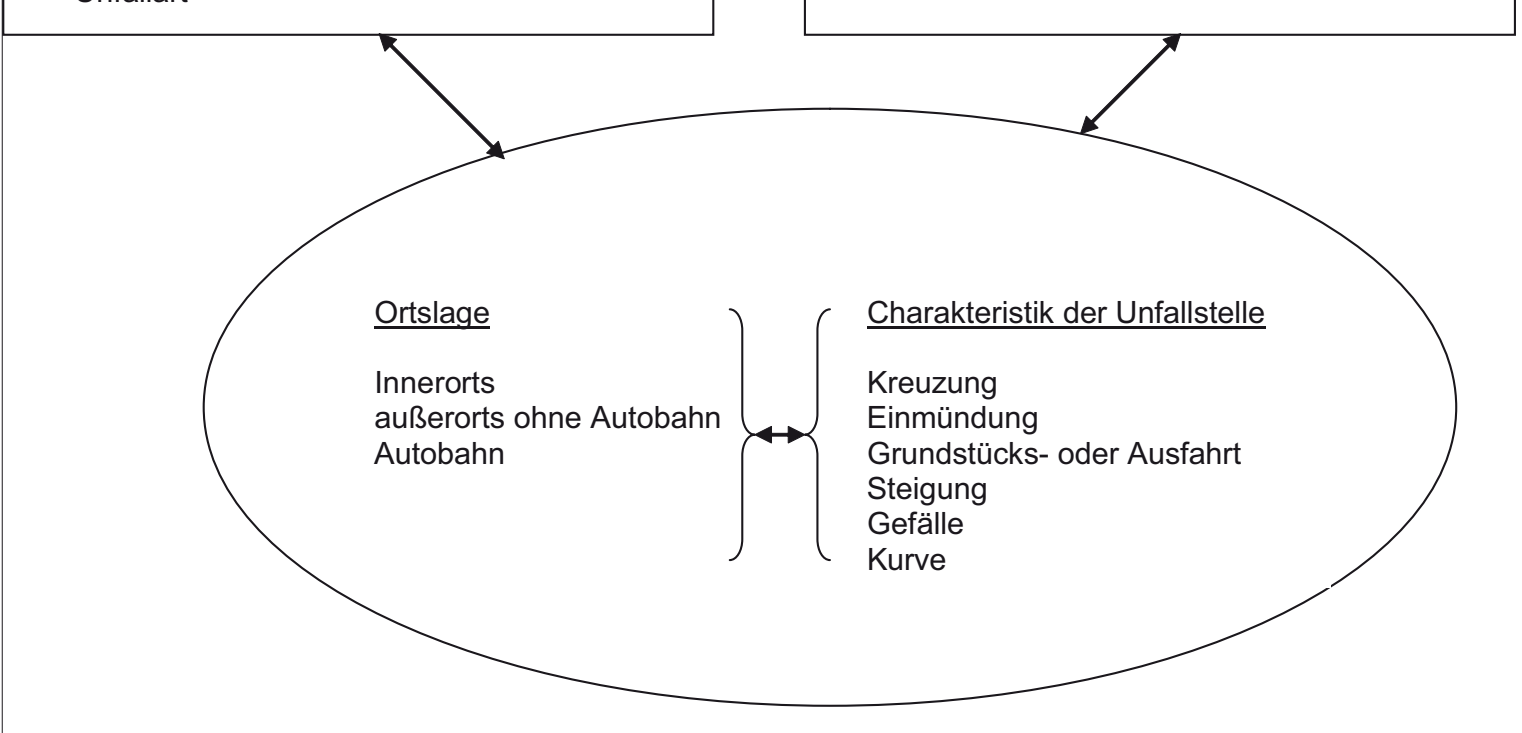

Abbildung 2-2: Verknüpfungsparameter zwischen Datenbasen von der TU-Dresden und TU-Berlin 


\section{Stand der Erkenntnisse - Literaturauswertung}

\subsection{Vorhandene Erkenntnisse aus dem Unfallgeschehen}

\subsubsection{Untersuchung des Unfallgeschehens}

Das Statistische Bundesamt in Wiesbaden erhält von jedem Statistischen Landesamt eines Bundeslandes monatlich die aufgenommenen Angaben zu allen polizeilich erfassten Straßenverkehrsunfällen, ausgenommen die personenbezogenen Daten. Die Landesämter füllen ihre Unfallstatistik mit Fahrzeugdaten des Kraftfahrzeugbundesamtes Flensburg-Dresden auf. Die gesammelten und aufbereiteten Daten werden $\mathrm{u}$. a. in einem statistischen Jahrbuch mit Grafiken und Tabellen veröffentlicht. Das digitale Exemplar steht im Internet kostenfrei zur Verfügung. Alle theoretisch möglichen Auswertungen sind in den Unterlagen nicht enthalten. Die ausgegebenen Tabellen und Grafiken sind von den dargestellten Größen jährlich gleich. Sind für eine Sonderuntersuchung bestimmte Daten erforderlich, kann eine Auswertung beantragt werden, die dann kostenpflichtig erwerbbar ist. Die statistischen Jahrbücher ermöglichen einen ersten Eindruck der Unfallsituation für bestimmte Verkehrsteilnehmer, Charakteristik und Besonderheiten der Unfallstelle, Ortslagen, Zeitpunkt oder Bedingungen zum Zeitpunkt des Unfalls.

In den letzten Jahren haben sich einige Untersuchungen verstärkt mit dem Unfallgeschehen von Motorradfahrern befasst. Der Einstieg in die Problematik erfolgte über die amtliche Unfallstatistik. In Tabelle 3-1 sind die drei umfangreichsten Untersuchungen benannt. Weiterhin sind sieben Bezugsgrößen angeben, die zeigen sollen, welche Auswertungen in den Untersuchungen vorgenommen wurden.
Meewes und Maier [Maier 2000] haben bei ihren Untersuchungen festgestellt, dass Motorradbenutzer ein siebenfach höheres Unfallrisiko als die übrigen Verkehrsteilnehmer besitzen. Für das Jahr 1997 berechnete sich für Motorradfahrer eine Getötetenrate von 92 GT je 1 Mrd. Motorradkilometer. Alle übrigen Verkehrs-teilnehmer fuhren mit einem Risiko von 13 GT je 1 Mrd. Kraftfahrzeugkilometer. Das Risiko für Mofa/Moped-Benutzer getötet zu werden, ist nur halb so groß wie bei Motorradfahrern. Bei einer Recherche stießen die Verfasser auf Untersuchungen aus den USA und Israel, die zu ähnlichen Ergebnissen kamen. Das Insurance Institute for Highway Safety ermittelte für das Jahr 1987 eine fünffach höhere Getötetenrate für Motorradfahrer im Vergleich zu den Autofahrern. In Israel ergab sich für das Jahr 1983 ein sechsfach höheres Risiko für Motorradfahrer.

Bei zwei Drittel der getöteten Motorradbenutzer im Jahr 1997 lag im Innerortsbereich (124 von 203 GT) sowie auf Landstraßen (470 von 723 GT) ein Selbstverschulden vor, auf Autobahnen sogar bei $86 \%$ der Getöteten ( 41 von 48 GT). Ein Selbstverschulden liegt dann vor, wenn der Motorradfahrer von der Polizei als Beteiligter 01 bestimmt wurde oder es zu einem Alleinunfall kam.

[Assing 2002] hat in seiner Untersuchung der amtlichen Unfallstatistik ein sehr breites Spektrum des Unfallgeschehens mit Motorradfahrern (ohne Mofa/Moped) von 1991 bis einschließlich 1999 dargestellt. Die Ergebnisse der Ausarbeitungen lassen sich mit folgenden Punkten zusammenfassen:

- Die Unfall- und Verunglücktenzahlen sind leicht angestiegen, was in erster Linie auf die Zunahme der

\begin{tabular}{|c|c|c|c|c|c|c|c|c|c|}
\hline \multicolumn{2}{|l|}{ Literaturquelle } & \multirow{2}{*}{$\begin{array}{l}\text { Unfälle } \\
\text { Zeitraum }\end{array}$} & \multicolumn{7}{|c|}{ Bezugsgrößen } \\
\hline Verfasser & Jahr & & Ortslage & EW & Bestand & Fahrleistung & |Beteiligte & Alter & Zeiten \\
\hline MEEWES/MAIER & 2000 & $1995-98$ & ja & nein & nein & ja & ja & nein & nein \\
\hline ASSING & 2002 & 1991-99 & ja & $\mathrm{Ja}$ & ja & ja & ja & ja & ja \\
\hline KOCH/KISLER & 2006 & 1991-04 & nein & nein & ja & ja & nein & ja & nein \\
\hline
\end{tabular}

Tabelle 3-1: Übersicht der vorhandenen Unfalluntersuchungen mit Motorradbeteiligungen 
leichten Unfälle zurückzuführen ist. Die Zahlen für Unfälle mit Getöteten und Schwerverletzten sind leicht rückläufig.

- Die mittlere Unfallschwere für Motorradunfälle mit Personenschaden ist um 14 \% von 142 auf 122 TDM zurückgegangen (Preisstand: 1998), liegt aber immer noch über dem Mittelwert aller Unfälle mit Personenschaden im Straßenverkehr (98 TDM, Rückgang von 1991 um $25 \%$ ).

- Die höchste Unfallschwere ergibt sich im Außerortsbereich (auf Autobahnen 164 TDM, auf Landstraßen 211 TDM). [Assing 2002] führt dies auf die höheren gefahrenen Geschwindigkeiten im Vergleich zu den Innerortsunfällen zurück. Gegenüber Autobahnen wird das Gefahrenpotenzial auf Landstraßen durch Kurven und Kreuzungen erhöht.

- Ein Drittel der Außerortsunfälle sind Alleinunfälle. Sie ereignen sich zu fast zwei Drittel in Kurvenbereichen. Als häufigster Unfalltyp zeigt sich der Fahrunfall, der insbesondere auf den Verlust der Kontrolle über das Fahrzeug hinweist. Die Schwere der Unfälle ergibt sich überwiegend aus dem Aufprall auf ein Hindernis neben der Fahrbahn.

- Die Zunahme der Unfallbeteiligung von Fahrern ab 35 Jahren wird auf den Anstieg des sehr starken Zuwachses des Bestandes in dieser Altersgruppe zurückgeführt. Auch die jungen Motorradfahrer (unter 18 Jahren), insbesondere beim Führen von Leichtkrafträdern, weisen ein hohes Bestandsrisiko auf. Die geringere Unfallschwere wird auf den hohen Anteil an Unfällen im Innerortsbereich zurückgeführt.

- War der Motorradfahrer bei Kollisionen Hauptschuldiger (Beteiligter 01) des Unfalls, ergab sich ein großer Anteil bei einem Zusammenstoß mit einem Fahrzeug das entgegenkommt oder vorausfährt (60 \%). Die Unfälle, bei denen andere Verkehrsteilnehmer als Hauptverursacher auftraten, ereigneten sich insbesondere an Knotenpunkten in Form von Abbiegeunfällen oder Einbiegen-/Kreuzen-Unfällen.

- Die vom allgemeinen Trend abweichende Entwicklung des Unfallgeschehens mit Motorrädern kann auf die unterschiedlichen Witterungsbedingungen einzelner Jahre zurückgeführt werden, da der Motorradverkehr insbesondere in den Monaten Mai bis September durch einen hohen Anteil an Freizeitverkehr geprägt ist. Bei zunehmenden Niederschlagsmengen sinkt die Anzahl der Motorradunfälle und Getöteten jedoch bei starker Zunahme der Streuung.

Ein Schwerpunkt der statistischen Unfallauswertung von [Koch 2006] stellte die altersbezogene Entwicklung der Motorradunfälle dar. Für den Untersuchungszeitraum von 1991 bis 2004 konnten folgende Erkenntnisse herausgearbeitet werden:

- Die Zahl der Verunglückten Motorradfahrer bewegte sich im Bereich zwischen 35.000 und 44.000 Verunglückten pro Jahr. Ein stetiger Rückgang ist nicht zu erkennen.

- Die Verunglücktenzahl der Altersgruppen 16-18 war zwischen 1991 und 1993 rückläufig (von 8.200 auf 6.300 V). Zwischen 1993 und 1999 erfolgte ein Anstieg auf fast 9.000 Verunglückte und sank anschließend bis 2004 stetig auf 5.900. Die Anzahl der Verunglückten der Altersgruppen 18-21, 21-25 und 25-30 sind über den gesamten Betrachtungszeitraum stets rückläufig gewesen

- Die Altergruppen 30-35 und 35-40 zeigen deutliche Zunahmen der Verunglücktenzahl von 1991 bis 1999 und anschließende Abnahmen bis 2004. Der Ausgangswert wurde nur bei der Altersgruppe 30-35 im letzten Jahr unterschritten

- In den Altersgruppen über 39 Jahre nahm die Verunglücktenzahl zwischen 1991 und 2003 beständig zu. Mit zunehmendem Alter nimmt der Anstieg jedoch ab. - Die Entwicklung der Bestandszahlen aller Krafträder nach Altersgruppen weist eine sehr große Ähnlichkeit zur Entwicklung der Verunglücktenzahlen auf. Für die Altersklassen ab 21 Jahre ist bei der bestandbezogenen Verunglücktenentwicklung ein Rückgang zu verzeichnen (Abbildung 3-1).

Die rückläufige Tendenz bei den Motorradbenutzern $a b$ 35 Jahren ergibt sich aus der geringeren Zunahme der Verunglücktenzahlen im Vergleich zum stärkeren Anstieg des Motorradbestandes. Bei der Altersgruppe 18 bis 21 Jahre berechnet sich ab dem Jahr 2000 eine Erhöhung der Verunglückten je 100.000 Motorräder, was auf den deutlichen Rückgang des Bestandes zurückzuführen ist. 


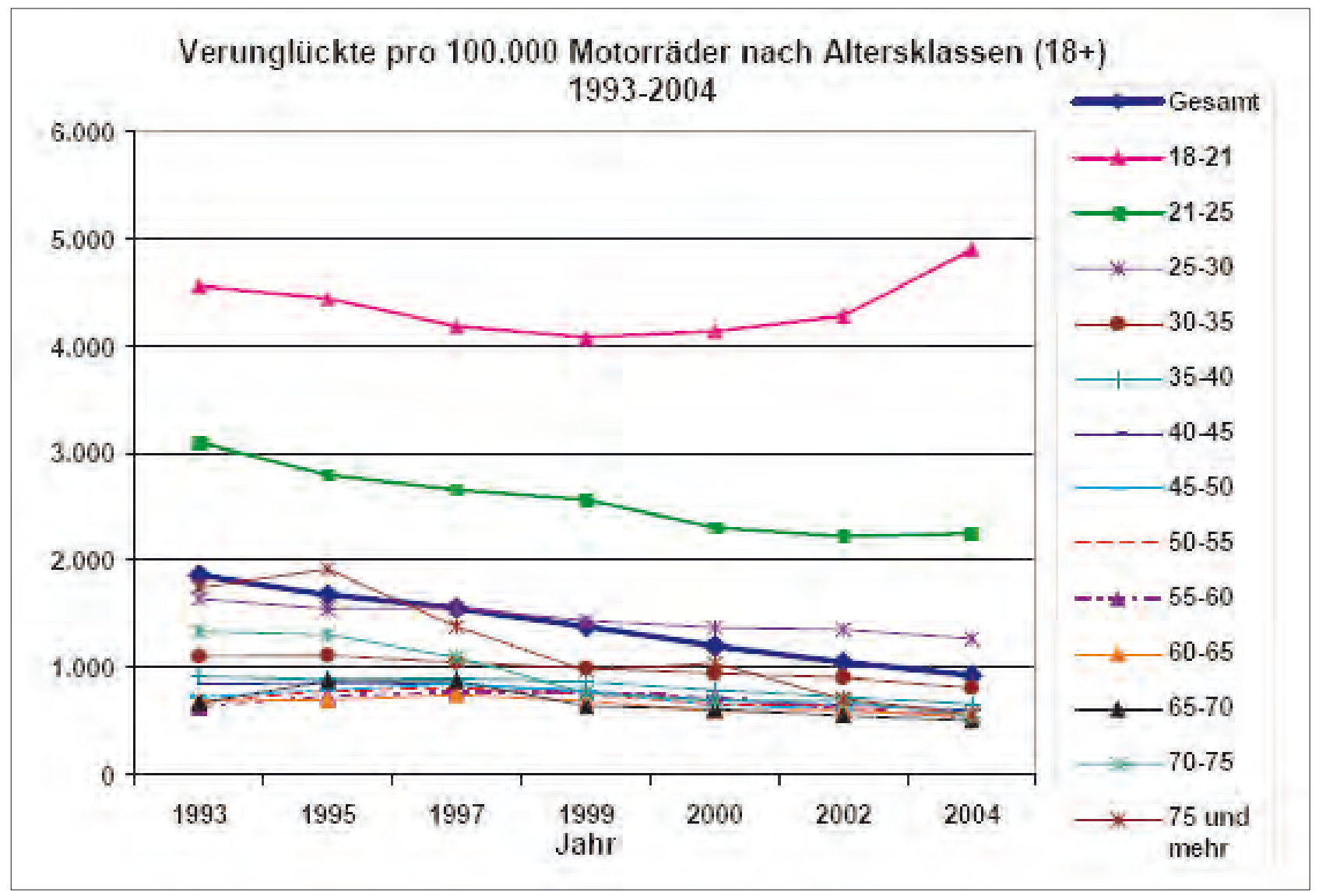

Abbildung 3-1: Bestandsabhängige Verunglücktenentwicklung differenziert nach Altersklassen [Koch 2006]

\subsubsection{Untersuchung des Unfallgeschehens mit Einbezug von Straßendaten}

In [Gerlach 2007] wurde eingehend der Einfluss von Straßeneigenschaften auf das Unfallgeschehen von motorisierten Zweirädern untersucht. Als Untersuchungsgebiet diente das Bundesland Rheinland-Pfalz, für dessen Straßennetz digitale Trassierungsdaten zur Verfügung standen.

In einem ersten Schritt wurden Unfallhäufungsstellen (UHS) und Unfallhäufungslinien (UHL) für Fahrunfälle mit Beteiligung von Motorrädern in Außerortslagen ausgenommen Knotenpunkte nach den Kriterien in [Hessen 2003] festgelegt. Basis ist dort die 5-Jahreskarte der Unfälle mit Personenschaden bzw. schwerem Personenschaden. Durch diese Vorgehensweise konnten 68 UHS und 63 UHL mit insgesamt 595 Unfällen identifiziert werden. In der weiteren Untersuchung wurden UHS und UHL überwiegend zusammen als Unfallhäufungen (UH) ohne Differenzierung betrachtet.

Anschließend erfolgte eine dreiteilige Auswertung in Bezug auf diese $\mathrm{UH}$ :

(1) Untersuchung des polizeilich erfassten Unfallgeschehens: Vergleich der Unfälle an UH mit allen Unfällen mit Motorradbeteiligung auf klassifizierten Straßen in Rheinland-Pfalz 1997-2002, bei denen eine Person verunglückt ist (10.311 Unfälle). (Der angegebene Zeitraum umfasst allerdings ein Jahr mehr als ein 5-Jahreszeitraum.)

(2) Untersuchung der Fahrbahngeometrie (Querneigung, Längsneigung, Kurvigkeit, Stetigkeit des Verlaufes) unmittelbar am Unfallort sowie in dem Bereich davor (punktuelle Betrachtung für die Unfälle an UH) 
(3) Vergleich der Fahrbahngeometrie von Streckenabschnitten, in denen sich die UH befinden, mit Streckenabschnitten ohne Unfallauffälligkeiten (linienhafte Betrachtung).

Die wichtigsten Ergebnisse dieser Auswertungen im Blick auf das vorliegende Projekt sind nachfolgend zusammengefasst:

\section{zu (1):}

- Die Unfallfolgen der Unfälle an UH sind im Durchschnitt deutlich schwerer als bei allen Unfällen. Dies ist bei Unfallhäufungen aller Verkehrsteilnehmer umgekehrt (Anm. der Verf.)

- Knapp 55 \% der Unfälle an UH ereignen sich am Wochenende. Im Vergleich zu allen Unfällen sind dies rund $20 \%$ mehr.

- Der Anstieg der Unfälle zu Saisonbeginn (April, Mai) fälltt bei den Unfällen an UH deutlicher aus als bei allen Unfällen.

- Etwa 60 \% der Unfälle an UH ereignen sich in einer Kurve.

- 2/3 der Unfälle an UH sind Alleinunfälle.

- Ca. 67 \% der Beteiligten der Unfälle an UH gehören zu der Altersgruppe der 20-40jährigen. Bei allen Unfällen stellt diese Gruppe etwa die Hälfte der Beteiligten dar. Aus dem Text wird nicht eindeutig ersichtlich, ob es sich bei den Beteiligten ausschließlich um Fahrer des PTW handelt. Dies ist aber anzunehmen.

- Rund $44 \%$ der Unfälle an UH weisen die Unfallart 8 - das Abkommen von der Fahrbahn nach rechts - auf. Das Abkommen von der Fahrbahn nach links, der Zusammenstoß mit einem entgegenkommenden Fahrzeug sowie der Unfall anderer Art stellen die weiteren wesentlichen Unfallarten für Unfälle an UH dar. Die übrigen Unfallarten spielen keine Rolle.

- Rund 1/4 aller Unfälle an UH verzeichnen einen Aufprall auf ein Hindernis. Der Anprall an eine Schutzplanke ist hierbei am stärksten vertreten, gefolgt von der Gruppe der sonstigen Hindernisse. Bei allen Unfällen liegt der Anteil mit Aufprall auf ein Hindernis unter $10 \%$.

\section{zu (2):}

- Bei 18 \% der Unfälle an UH entspricht die Querneigung nicht den Anforderungen der Fahrdyna. ik (Fahrbahnrand der Kurvenaußenseite nicht höher als Innenseite).
- Das Verhältnis der der Unfälle an UH auf Gefällestrecken zu den Unfällen an UH auf Steigungsstrecken beträgt 60 zu 40. Werden nur Gefälle- und Steigungsabschnitte über $4 \%$ bis $10 \%$ betrachtet, zeigt sich auf den Gefälleabschnitten eine fast doppelt so hohe Anzahl der Unfälle an UH wie bei den Steigungsabschnitten. Die Anzahl der Unfälle auf ebenen Streckenabschnitten beträgt nur $3 \%$. In Abbildung 3-2 sind die Ergebnisse einer kombinierten Betrachtung von Längsneigung, Querneigung und Kurvenausrichtung dargestellt.

- Es ergeben sich zum einen die meisten Unfälle bei Linkskurven im Gefälle (130), gefolgt von Rechtskurven im Gefälle (105), und zum anderen ist der Einfluss von fahrdynamisch ungünstigen Querneigungen vornehmlich bei den Linkskurven (14 plus 28 Unfälle) zu erkennen. Fazit: Gefällestrecken und hier insbesondere die Linkskurven bilden ein besonderes Risikopotential für Motorradfahrer einen Unfall zu erleiden.

- Streckenabschnitte mit einer Kurvigkeit über 200 gon/km besitzen einen Anteil von knapp $60 \%$ an allen Unfallstrecken, beinhalten jedoch $78 \%$ der Unfälle an UH. Bei Betrachtung einzelner Kurvigkeitsklassen (z. B. 200-300 gon/km) zeigt sich eine noch höhere Diskrepanz. Der Einfluss der Kurvigkeit auf das Unfallgeschehen ist ersichtlich.

- Für das Kriterium Stetigkeit im Verlauf wurden verschiedene Intervalle von 0 bis $300 \mathrm{~m}$ vor der Unfallstelle (aufgenommene Unfallposition) auf Veränderungen (Radius, Richtungswechsel) analysiert. 2/3 der Unfallstrecken weisen im Bereich von 200 bis $300 \mathrm{~m}$ mindestens eine Verlaufsänderung auf. Über 50 \% der Strecken verzeichnen bereits im unmittelbaren Vorfeld der Unfallstelle (bis $50 \mathrm{~m}$ ) eine Verlaufsänderung. Im Mittel besitzt eine Strecke für den Entfernungsbereich von $300 \mathrm{~m}$ bereits drei Änderungen im Verlauf. Fazit: In der Regel handelt es sich um unstetige Verläufe.

\section{zu 3:}

- Nachweis eines Zusammenhanges von Unfallgeschehen und Trassierungsparametern für Streckenabschnitte zwischen zwei Netzknoten mit einer Kurvigkeit größer 200 gon/km, mehr als 15 Änderungen des Streckenverlaufes pro km, einem Geradenanteil kleiner $50 \%$ und einer Streckenlänge größer $2 \mathrm{~km}$. 


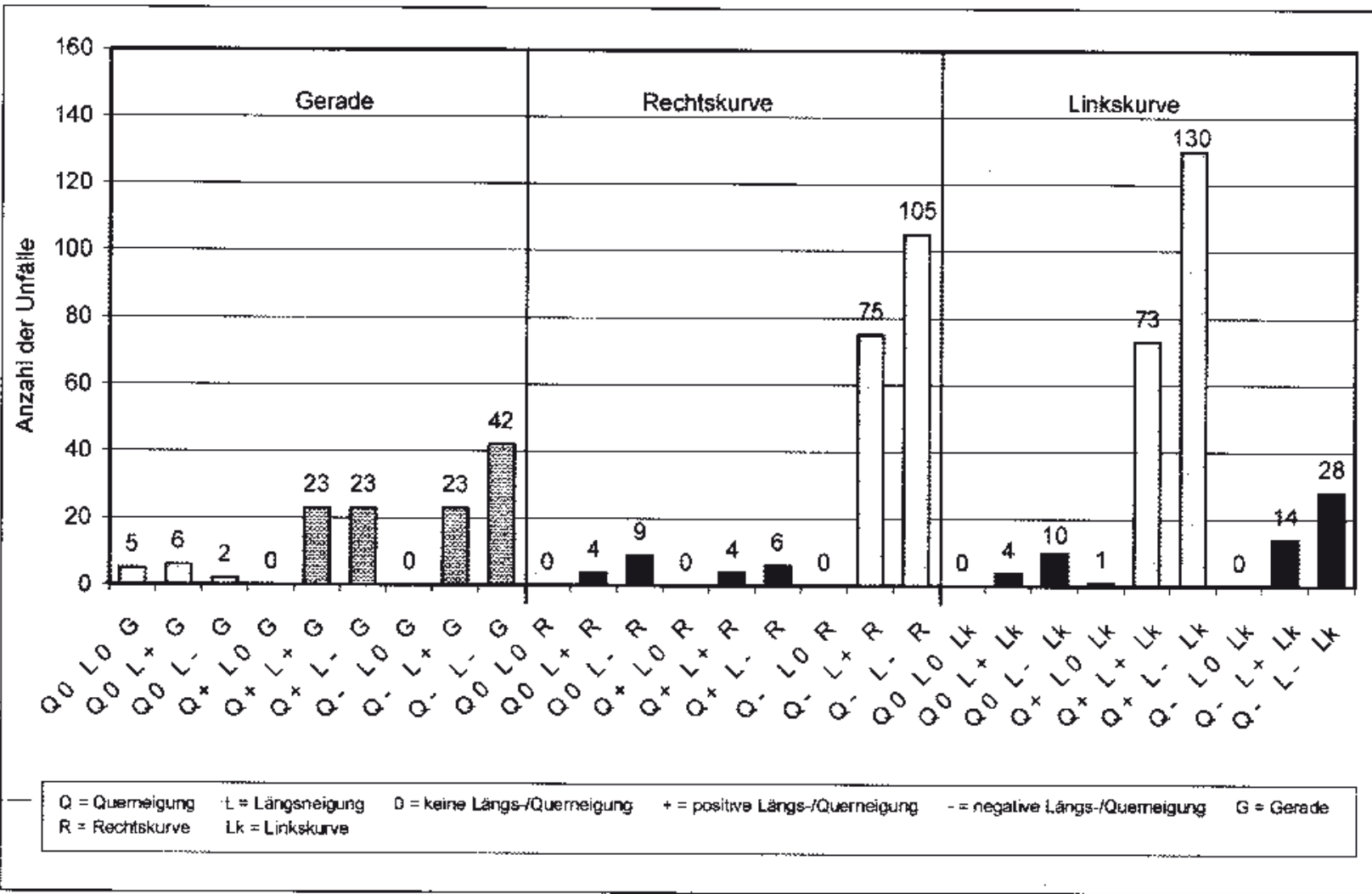

Abbildung 3-2: Kombinierte Betrachtung von Längsneigung, Querneigung und Kurvenausrichtung in [Gerlach 2007]

\subsection{Literaturstudie - Gefahren für den Motorradfahrer aus Sicht der Fahr- zeugtechnik}

Bei der Betrachtung der Gefahren für Motorradfahrer aus fahrzeugtechnischer Sicht sollten zunächst die Besonderheiten des Motorradfahrens berücksichtigt werden [Stoffregen 1995]. Aus fahrphysikalischer Sicht handelt es sich beim Motorrad oder generell beim Zweirad um ein Einspurfahrzeug, welches sich immer in einem labilen Gleichgewichtszustand befindet. Im Stand kippt es ohne Stützwirkung um, während der Fahrt wird es rein dynamisch stabilisiert; dabei spielen die Kreiselkräfte der sich drehenden Räder eine Rolle. Damit hängt auch die Eigenart des Lenkens zusammen. Motorräder werden nicht durch den Lenkeinschlag an sich gelenkt, sondern der Lenkeinschlag dient nur zum Einleiten der für die Kurvenfahrt notwendigen Schräglage. Durch die Schräglage halten sich die in der Kurve auftretende Fliehkraft und die Schwerkraft im Gleichgewicht [Stoffregen 1995]. Aufgrund der Besonderheiten des Einspurfahrzeugs als dynamisches System aus Fahrer und Fahrzeug muss der Fahrer in die fahrdynamische Betrachtung mit einbezogen werden. Anders als etwa beim Pkw trägt der Fahrer allein schon mit über 20 \% zum Gesamtgewicht bei. Fahrer und Beifahrer beeinflussen darüber hinaus durch Gewicht, Sitzposition, Bewegungen und Feder-Dämpfer-Eigenschaften des menschlichen Körpers aktiv das Fahrverhalten.

Wegen des labilen Zustandes kann die fahrdynamische Stabilität des Systems Einspurfahrzeug schon durch geringe Störgrößen wie Seitenwind oder unbewusste und hastige Bewegungen des Fahrers beeinträchtigt werden, was zu gefährlichen Situationen führen kann. Diese Einflüsse müssen vom Fahrer permanent ausgeglichen werden; das geschieht hoch trainiert, meist unbewusst. 
Auch bei der Kurvenfahrt in Schräglage befindet sich das System des Einspurfahrzeugs in einem instabilen Gleichgewichtszustand, welcher vom Fahrer fein ausbalanciert werden muss. Das Gesamtsystem ist hier äußerst empfindlich, Störgrößen wie abrupte Geschwindigkeitsänderung, schnelle Änderung des Kurvenradius, plötzliche Änderungen des Fahrbahnreibwertes oder auftretender Rollsplitt führen sehr leicht zum Sturz.

Anders als etwa beim Pkw sind auch die dynamischen Radlastveränderungen beim Motorrad aufgrund des ungünstigeren Verhältnisses von Schwerpunkthöhe und Radstand viel stärker ausgeprägt. Der Schwerpunkt liegt wegen der hohen Sitzposition der Aufsassen in der Regel höher als beim Pkw, während der Radstand beim Motorrad deutlich kleiner ist.

Die stärker ausgeprägten dynamischen Radlaständerungen sind ein Grund dafür, weshalb das Bremsen eines der schwierigsten Manöver beim Motorradfahren ist. Leider herrscht heute standardmäßig immer noch die getrennt zu betätigende Vorder- und Hinterradbremse vor. Dabei müssen zwei voneinander unabhängige Bremskreise - für die Vorderrad- und die Hinterradbremse - bedient werden. Die Schwierigkeit für den Motorradfahrer besteht darin, dass er wegen der dynamischen Radlastverlagerung nach vorne aufgrund der Fahrzeugverzögerung die Bremskraftverteilung zwischen Vorder- und Hinterrad bei zunehmender Verzögerung stetig neu einstellen muss. Dies stellt schon bei einfacher Geradeausfahrt eine sehr schwierige Regelaufgabe für den Fahrer dar, um den kürzestmöglichen Bremsweg zu erreichen [BASt 1996]. Die Bremskraft muss zunehmend nach vorn verlagert werden. Dabei besteht die größte Gefahr für Motorradfahrer in der Vorderradblockade: Die zur Stabilisierung des Kraftrades wichtigen Kreiselkräfte des Vorderrades entfallen, die Seitenkräfte gehen gegen Null und der Motorradfahrer stürzt nach kurzer Zeit. Die Blockade des Hinterrades ist gegenüber der des Vorderrades weniger kritisch, stellt aber auch ein Gefahrenpotenzial dar. Abhilfe schaffen hier Kombibremssysteme, bei denen bei Betätigung des Handbremshebels automatisch auch das Hinterrad mit abgebremst wird. Einige dieser Systeme verfügen auch über eine automatische Bremskraftverteilung zwischen Vorder- und Hinterrad (z. B. Dual-CBS von Honda).

Beim Bremsen in der Kurve kommen noch weitere Probleme hinzu. Eine Störung der Seitenführungskraft führt zur Störung des Kräftegleichgewichts zwischen Fliehkraft und Gewichtskraft. Bei Kurvenfahrt ergibt sich außerdem eine Verschiebung der Reifenaufstandspunkte aus der Mittelebene heraus. Der so entstehende Lenkrollradius führt zusammen mit den Bremskräften zu so genannten Bremslenkmomenten, deren Kompensation bis heute dem Fahrer überlassen ist. Am Hinterrad stellt diese Aufgabe aufgrund des großen Nachlaufs kein Problem dar. Das Auftreten am Vorderrad ist dagegen nur bei geringen Umfangskräften, -gradienten und -schwankungen beherrschbar. In Panik- oder Schrecksituationen und bei ABS-Regelung ist der Fahrer mit dem Aufbringen des Gegenmomentes am Lenker zur Kurshaltung überfordert. Die Folgen sind das Einlenken in die Kurve, die daraus resultierende Aufstellneigung und das anschließende mehr oder weniger tangentiale Verlassen des Kurvenradiusses (ohne ABS) und massive Lenkunruhen (mit ABS-Regelung).

Der Gewinn an objektiver, aktiver Sicherheit durch ABS beim Motorrad ist deutlich höher einzuschätzen als bei Mehrspurfahrzeugen. Für einen Pkw-Fahrer etwa sind blockierende Räder nicht unmittelbar Unfall verursachend, da das Fahrzeug kippstabil bleibt. Bei Mehrspurfahrzeugen wird das ABS eingesetzt, um die Lenkbarkeit des Fahrzeuges auch bei hohen Verzögerungen sicherzustellen. Für den Motorradfahrer bedeutet dagegen ein blockiertes Vorderrad, wie bereits erwähnt, den unmittelbar drohenden Sturz. Das Motorrad-ABS hat daher die wesentliche Aufgabe, Stürze durch Radblockaden zu vermeiden.

In dem BASt-Bericht F18 (1996) wurde das Bremsverhalten von Fahrern von Motorrädern mit und ohne ABS untersucht. Dazu wurden Fahrversuche mit erfahrenen und wenig erfahrenen Motorradfahrern durchgeführt. Übergeordnetes Thema dieser Untersuchung war die Auswirkung der Unterstützung der Bremsung 
mit aktuell verfügbaren ABS-Anlagen auf die Sicherheit von Motorradfahrern. Dazu wurden mehrere Teilziele definiert:

- Darstellung des technischen Leistungspotenzials von ABS - ABS-Anlagen werden stetig weiterentwickelt. Wirkungsvolle, schnelle Verbesserungen sind schon durch Implementierung anderer Steuer- und Regelalgorithmen in elektronischen Steuereinheiten bereits existierender Systeme möglich. Betrachtet wird hier der zum Zeitpunkt des Projektstarts neueste Stand von ABS-Systemen. Besondere Beachtung gilt der Kurvenbremsung.

- Bremsleistungspotenzial erfahrener Fahrer

- Vergleich des technischen Leistungspotenzials des ABS mit der Bremsleistungsfähigkeit mehrerer erfahrener Motorradfahrer.

- Untersuchung des realen Fahrerverhaltens

- Möglichkeit zur Entdeckung besonderer Fahrsituationen und zur Untersuchung des Fahrerverhaltens in „abnormalen“ (evtl. kritischen) Situationen. Nutzung der Erkenntnisse zur Ableitung von Aussagen über die vorliegende Übung und die Fähigkeiten von Fahrern.

- Fahrerverhalten mit und ohne ABS

- Vergleich der Fahrerleistungen mit und ohne ABS anhand messbarer Größen mit dem Ziel, Hinweise auf Veränderungen des Fahrerverhaltens durch ABS zu erhalten.

Das Fahrerverhalten wurde wirklichkeitsnah untersucht. Dazu wurden Fahrversuche mit den Testpersonen überwiegend im öffentlichen Straßenverkehr durchgeführt. Es zeigte sich eine starke Änderung des Bremsverhaltens bei wechselndem Nässezustand der Fahrbahn. Der Fahrbahnnässezustand hat den größten Einfluss auf die für globale Betrachtungen des Bremsverhaltens charakteristischen Verzögerungswerte.

Unerfahrene Versuchspersonen bremsten im öffentlichen Verkehr seltener als die erfahrenen.

Eine nennenswerte Anzahl von Bremsungen fand bei Kurvenfahrt statt, womit die Kurvenbremsung schon für das normale Bremsverhalten relevant ist.
Selbst normalerweise beide Bremsen betätigende Versuchspersonen betätigten bei Kurvenfahrt nur einen Bremskreis, vorwiegend in wenig belastenden Situationen. Dies birgt ein erhöhtes Risiko bei plötzlichen Ereignissen, dann verbunden mit der Gefahr der Überbremsung des schon betätigten Bremskreises durch dessen voreilende Betätigung. Fehlender Blockierschutz und die Aufgabe der Bremskraftverteilung überfordern somit den Fahrer spätestens in kritischen Situationen, in denen meist noch andere Verkehrsteilnehmer zugegen sind, deren Reaktionen und Handlungen zusätzliche Aufmerksamkeit vom Motorradfahrer erfordern.

Die ständig gegenwärtige Angst der Versuchspersonen vor Stürzen insbesondere durch Vorderradblockade wirkte sich stark nachteilig auf das Bremsverhalten aus. Sie verhinderte das Erreichen der maximal möglichen Verzögerung, die vor allem von den Vorderradbremskräften abhängt. Auch werden bei Hinterradblockade gleichzeitig beide Bremsen gelöst, obwohl am Vorderrad noch eine Erhöhung der Bremskraft möglich wäre. Motorradfahrer sollten somit auf das Vorhandensein von ABS bewusst reagieren, um höhere Verzögerungen in kritischen Situationen zu erreichen und bei Hinterradblockade nicht beide Bremsen zu lösen.

Schon eine mit geringem Aufwand realisierbare Kombibremse mit konstruktiv vorgegebener Bremskraftverteilung und voreilend überbremsten Hinterrad kann viele der beobachteten Nachteile beseitigen.

Bei bestimmten Versuchspersonen häuften sich Hinweise auf Veränderungen des Bremsverhaltens durch ABS. Die höheren erreichten charakteristischen Verzögerungswerte deuten auf eine geringere Angst vor einer Vorderradblockade hin. Zwar erreichten erfahrene Fahrer ohne ABS bei idealen Bedingungen (geringe Geschwindigkeit, Vorhersehbarkeit, Geradeausfahrt, trockene Fahrbahn, Eingewöhnung) etwas höhere Verzögerungen als mit $A B S$, trifft jedoch schon eine dieser Bedingungen nicht $\mathrm{zu}$, wie im realen Verkehrsgeschehen anzunehmen, sind die Verzögerungen ohne ABS niedriger als diejenigen mit ABS. 
ABS bietet vor allem für wenig erfahrene Motorradfahrer die Voraussetzung, in kritischen Situationen fahrstabil hohe Verzögerungen zu erreichen.

Mit dem ABS des Versuchsmotorrades (BMW R 1100 RS) konnten Vollbremsungen bei Kurvenfahrt ohne nennenswerte Kursabweichung und ohne Sturz absolviert werden. Je nach Betätigung der Bremskreise kommt es aber bei ABS-Regelung zu unterschiedlich starken Lenkmoment- und Lenkwinkelschwankungen. Das verwendete ABS ist somit zumindest eingeschränkt kurventauglich. Die Weiterentwicklung zu einem voll kurventauglichen ABS würde also einen weiteren deutlichen Sicherheitsgewinn darstellen.

In einer weiteren Studie führte die Bundesanstalt für Straßenwesen eine Untersuchung der Bremsung mit verschiedenen Kraftradbremssystemen durch [BASt 2004]. Dabei erhielten männliche Testpersonen die Aufgabe, Bremsungen mit 5 verschiedenen Bremssystemen durchzuführen (Standard- und Kombibremse jeweils mit und ohne ABS sowie blockiergeschützte Kombibremse mit Einhebel-Bedienung). Während der Tests wurden die damit erzielten Bremswege sowie die Belastungs- und Beanspruchungsgrößen der Fahrer aufgezeichnet (Herzschlagfrequenz, Muskelanspannung). Für größtmögliche Vergleichbarkeit wurden alle Bremssysteme an einem Motorrad realisiert. Die Versuche wurden auf einem Testgelände mit nassem Asphalt durchgeführt, mit Geradeausbremsungen aus 60 und $90 \mathrm{~km} / \mathrm{h}$ und Kurvenbremsungen aus $50 \mathrm{~km} / \mathrm{h}$.

Die erzielten Bremswege mit ABS waren nachweislich kürzer als ohne. Dies ist vor allem auf Unterschiede in der Anfangsphase einer Bremsung zurückzuführen: die während der Anfangsphase verstreichende Zeit ohne Bremsverzögerung steigt bei Bremsungen ohne ABS überproportional mit der Ausgangsgeschwindigkeit, dies gilt vor allem bei Kurvenbremsungen. Ein signifikanter Unterschied zwischen Standard- und Kombibremse konnte nicht festgestellt werden. Darüber hinaus hat die Bedienung einer blockiergeschützten Kombibremse mit nur einem Bremshebel keinerlei Nachteil gegenüber einer konventionellen Zweihebel-Bedienung. Bei Bremsungen ohne ABS wurde ein deutlich stärkerer Anstieg der Herzschlagfrequenz ermittelt als mit ABS, gleiches gilt für die Muskelanspannung, welche als Belastungsgröße herangezogen wurde. Zu Stürzen während des Bremsvorgangs kam es nur bei Bremsungen ohne ABS. Auch hier zeigt sich also wieder der deutliche Sicherheitsgewinn durch ABS.

Wissenschaftler des österreichischen Kuratoriums für Verkehrssicherheit in Wien fanden darüber hinaus einen psychologischen Effekt des ABS heraus [Winkelbauer und Vavryn 2002]. Demnach genügt bereits das Wissen des Motorradfahrers, durch das ABS vor einem Sturz wegen blockierender Räder geschützt zu sein, damit er stärker verzögert als ohne ABS. Dadurch verkürzen sich die Bremswege bereits bei Bremsungen, bei denen das ABS noch gar nicht eingegriffen hätte.

Hierzu wurde eine Felduntersuchung mit Motorradfahrern durchgeführt, die nacheinander Bremsversuche mit dem eigenen Motorrad und mit einem bereitgestellten Motorrad mit ABS fuhren. Zusammengefasst ergeben sich aus dieser Untersuchung folgende Schlussfolgerungen:

- Motorradfahrer bremsen auf einem Motorrad mit ABS um durchschnittlich $1,24 \mathrm{~m} / \mathrm{s}^{2}$ besser als mit dem eigenen Motorrad ohne ABS. Dabei verfügten die Versuchspersonen nur über wenige Minuten Fahrerfahrung auf dem bereitgestellten ABS-Motorrad.

- Damit Motorradfahrer mit ABS effizient bremsen können, bedarf es einer entsprechenden Erklärung der Funktion und richtigen Bedienung. Ist diese erfolgt, ist es möglich, mit relativ wenig Übung unter fachlicher Anleitung befriedigende Bremsungen zuwege zu bringen.

- Da mit einer - wenn auch langsamen - Verbreitung von ABS bei Motorrädern zu rechnen ist, sollte die richtige Bedienung von ABS in Theorie und Praxis in den Lehrplan für die Fahrschulausbildung aufgenommen werden. Aus den Erfahrungen mit den Rückmeldungen der Versuchspersonen zeigt sich, dass in vielen Fällen damit auch zum Abbau von Vorurteilen gegenüber ABS beigetragen und somit ein wesentlicher Beitrag für eine weitere Verbreitung geleistet werden könnte. 
Die Wirksamkeit des ABS bei Motorrädern wird auch in [Gwehenberger 2006] verdeutlicht. Die Autoren stellen eine Untersuchung von 200 Schwerstunfällen mit Motorradbeteiligung der Allianz Versicherung vor. Auch hier werden die Vorteile des ABS hervorgehoben: Es stabilisiert die Bremsung, verkürzt den Bremsweg, verhindert das Überbremsen des Vorderrades und somit den gefährlichen Sturz beim Bremsen. Darüber hinaus sorgt es für eine geringere Stressbelastung des Motorradfahrers beim Bremsen, insbesondere in Grenz- und Notsituationen. Nach der Analyse der 200 Unfälle hätte ABS in 8 bis $17 \%$ der Fälle den Unfall vermeiden oder zumindest die Unfallfolgen vermindern können. Hochgerechnet auf Deutschland könnten bei flächendeckender ABS-Ausrüstung etwa 100 getötete und über 1.000 schwer verletzte Motorradfahrer pro Jahr vermieden werden. Ebenso wäre ein Rückgang um mehr als 1.000 verunglückte Motorradfahrer mit leichten Verletzungen zu erwarten. Die Allianz Versicherungs-AG gewährt daraufhin seit dem 1. April 2005 reduzierte Beiträge für die Kfz-Haftpflichtversicherung für Motorräder mit ABS. Gleichzeitig forderte sie die Motorradhersteller auf, ABS künftig serienmäßig einzubauen [Allianz 2006]. Diese Initiative scheint nun erste Früchte zu tragen. Alle großen Zweiradhersteller bieten inzwischen ABS serienmäßig oder optional an, und etwa jedes fünfte neu zugelassene Motorrad ist heute mit $A B S$ ausgestattet.

Nach Kramlich und Sporner [Kramlich 2000] treten die häufigsten Verletzungen bei Motorradfahrern an Oberund Unterschenkeln sowie am Kniegelenk auf. Ursache dafür sind hinderliche Motorradteile beim Lösen des Fahrers vom Motorrad bei einer Kollision und der Zusammenprall mit dem Unfallgegner. Diese Verletzungen sind mitunter kompliziert und langwierig im Heilungsverlauf, stellen aber in der Regel kein tödliches Risiko dar. Tödliche Gefahren für den Motorradfahrer bestehen dagegen besonders häufig beim Kopfaufprall, zum Beispiel beim Anprall an die steife Pkw-Dachkante bei Seitenkollisionen. Auch bei Alleinunfällen besteht eine erhebliche Gefährdung des Kopfes durch schwere Anstöße zum Beispiel an Schutzplanken oder Begrenzungspfosten. Kramlich und Sporner stellen für die Entwicklung der passiven Sicherheit von Motorrädern folgende Punkte heraus:
- Kontinuierliche Weiterentwicklung der Helmtechnik, besonders seit Einführung der ECE-Norm auch bei Helmen der unteren Preisklasse hervorragendes Sicherheitsniveau

- Wandel bei Motorradfahrerkleidung: schwarze Lederjacken und -hosen mit nur angedeuteten Prallpolstern sind nur noch bei Puristen zu finden. Das Straßenbild wird bestimmt durch weitgehend wasserdichte Textiljacken in bunten, gut sichtbaren Farben, aus abriebfestem High-Tech-Gewebe mit Kevlarverstärkung. - Für CE-Protektoren bestehen seit 1999 verbindliche Vorschriften über deren Leistungsfähigkeit

- Erste Ansätze bei der Optimierung von Motorradkomponenten wie Verkleidung, Tank, Fußrasten, Lenker, um bei einer Kollision möglichst kontrolliertes, komplikationsfreies Lösen des Fahrers vom Fahrzeug zu gewährleisten.

- Studien zeigen ein beachtliches Wirkpotenzial bei Airbags für Motorräder, hinsichtlich Bewegungskinematik des Motorradfahrers bei einer Kollision sowie als Prallpolster. Dies ist bisher nur bei Honda als Option für eines der Fahrzeuge im Angebot.

Kramlich und Sporner weisen darauf hin, dass Verbesserungen am Motorrad selbst nur Wirkung zeigen können, wenn der Fahrer mit dem Motorrad in einigermaßen aufrechter Sitzhaltung gegen den Pkw prallt. In 20 \% aller Motorrad/Pkw-Kollisionen aber stürzt der Motorradfahrer weit vor dem Zusammenprall. Ein optimales Schutzpotenzial für Motorradfahrer kann daher nur im Zusammenspiel mit aktiven Sicherheitselementen geschaffen werden. Im Bereich der aktiven Sicherheit werden dabei zwei Hauptgebiete der Motorradsicherheit unterschieden:

- Ausbildung, Fortbildung, Aufklärung des Motorradfahrers und der anderen Verkehrsteilnehmer

- Fahrzeugkomponenten, die auch in Krisensituationen möglichst stabile Fahrweise ermöglichen.

Für den Fortschritt in der aktiven Sicherheit werden folgende Punkte herausgehoben:

- Einführung des Stufenführerscheins zur Verhinderung, dass junge Fahranfänger mit leistungsstarken, oft schwer 
beherrschbaren Hochgeschwindigkeitsmotorrädern ihr Leben und das anderer Verkehrsteilnehmer gefährden.

- Fahrwerke moderner Motorradgenerationen bieten hervorragende Fahreigenschaften. Deutliche Fortschritte wurden hinsichtlich Kurvenstabilität und Stabilität bei hohen Geschwindigkeiten erreicht. Die gewonnene Stabilität gaukelt dem Fahrer aber „gefährliche Scheinsicherheit "vor. Bei schlechter Fahrbahn gibt es wenig Rückmeldung, so dass jegliches Gefühl für Grenzbereiche und reduzierte Bodenhaftung praktisch verloren geht.

- Motorradreifen neuester Generation mit sehr guter Bodenhaftung auch bei widrigen Wetterbedingungen.

- Einführung moderner, immer wirkungsvollerer Bremsen (Kombibremse). Aber: Enorme Bremsleistungen heutiger moderner Bremsanlagen sind in Schrecksituationen kaum beherrschbar, als Folge treten Sturz oder verschenken von wertvollem Bremsweg auf.

Als Abhilfe hinsichtlich des letzten Punktes wird das ABS genannt, welches das sturzverursachende Blockieren des Vorderrades verhindert. Durch einen Sturz vor der Kollision kommt es bei Motorrad/Pkw-Kollisionen zu besonders schweren Verletzungsfolgen des Motorradfahrers. Solche Unfälle wären in den meisten Fällen durch ABS vermeidbar gewesen bzw. die Unfallfolgen wären mit ABS deutlich geringer. Das Wirkpotenzial von ABS ist aber nicht nur auf Unfälle mit Sturz beschränkt: aus Angst vor drohender Vorderradblockade schöpft der Fahrer das mögliche Bremspotenzial nicht zu 100 \% aus oder er löst die Bremse für einen Sekundenbruchteil und verschenkt somit wertvollen Bremsweg. Die Möglichkeit zur Ausnutzung des kompletten Bremspotenzials hätte eine deutlich geringere Kollisionsgeschwindigkeit und somit erheblich geringere Verletzungen zur Folge. Der Zusammenstoß von Motorrad und Fahrer in aufrechter Position mit dem Pkw durch ABS ist zudem ideale Voraussetzung für den optimalen Einsatz von passiven Schutzmaßnahmen wie zum Beispiel den Airbag. Kramlich und Sporner kommen daher zu dem Schluss, dass die Voraussetzung für eine spürbare Reduzierung der Zahl der getöteten oder schwer verletzten Motorradfahrer eine ausgewogene, optimale Kombination von aktiven und passiven Sicherheitselementen ist. Eines allein kann nur sehr wenig oder gar nichts bewirken.
Zahlreiche Untersuchungen und Veröffentlichungen gibt es auch zum Motorradairbag. Rücker und Berg [Rücker 2005] berichten über die Erkenntnisse von Crashtests der DEKRA mit einem selbst entwickelten Motorradairbag. Demnach sind Kollisionen mit einem Anprall des Motorradfahrer-Kopfes an die seitliche Dachkante eines Pkw eine häufig tödliche Unfallkonstellation. 2002 wurden erste Full-Scale-Versuche der DEKRA-Unfallforschung mit einem selbst entwickelten Airbag-Prototyp für ein mittelgroßes Tourenmotorrad durchgeführt. Dabei prallte das Motorrad mit 48 km/h rechtwinklig gegen die Seite eines stehenden $\mathrm{Pkw}$, in Anlehnung an ISO 13232, Konfiguration 413. Der verwendete Dummy war ein Hybrid III 50 \% male. Ein direkter Anprall des Motorradfahrer-Kopfes an die PkwDachkante wurde dabei durch den Airbag vermieden, im weiteren Bewegungsablauf gelangte der gesamte Oberkörper über die Dachkante aus dem gegebenen Gefahrenbereich heraus. Durch den Airbag wurden alle Belastungen (Kopfverzögerung, Halskräfte und -momente) reduziert. Anfang 2004 wurden weitere Tests durchgeführt, mit der gleichen Konfiguration, diesmal aber mit einem mit $24 \mathrm{~km} / \mathrm{h}$ fahrenden Pkw. Der verwendete Dummy war diesmal der spezielle Motorradfahrerdummy MATD. Wieder wurde durch den Airbag der Kopfanprall vermieden, wegen der Querbewegung des Pkw kam es aber nicht zum Aufgleiten auf das Dach. Auch hier wurden die gemessenen Dummybelastungen durch den Airbag verringert, allerdings gab es hier ein höheres Halsbiegemoment und eine höhere Halsdruckkraft mit dem Airbag. In beiden Versuchsreihen lagen aber die Belastungswerte mit Airbag deutlich unterhalb der Grenzwerte. In der Gesamtbetrachtung wurde daher ein erhebliches Nutzenpotenzial für den eingesetzten Airbagprototyp festgestellt.

Honda führte 2006 als erster Hersteller den Motorradairbag optional bei der Luxustourenmaschine Gold Wing ein [Kuroe 2005]. In diesem Airbag steckt jahrelange Forschungs- und Entwicklungsarbeit. Eine Übernahme der Pkw-Technologie war nicht möglich, da der Fahrer anders als im Auto je nach Motorradtyp eine andere Haltung einnimmt. Für die Einführung des Airbags bei der Gold Wing sprechen folgende Punkte: 
- aufrechte Fahrerposition, dadurch Platz vor dem Fahrer zum Auslösen des Airbags

- niedriger Schwerpunkt und hohe Masse, dadurch geringeres Abheben und Gieren während des Aufpralls.

Zur Auslösung des Airbags dienen vier Sensoren in der Gabel. Anhand der Signale erkennt das Steuergerät, ob der Airbag ausgelöst werden muss oder ob es sich beispielsweise nur um eine Bordsteinüberfahrt handelt. Ein erhöhtes Risiko durch technische Mängel besteht bei Motorrädern gegenüber Pkws offensichtlich nicht. Demnach achten Motorradfahrer stärker auf die Sicherheit ihres Fahrzeugs als Autofahrer. Das ergab die Auswertung der Hauptuntersuchungen im Jahr 2005 des TÜV Nord [TÜV 2006]. Danach wurden 74,6 \% der Motorräder ohne Mängel beim TÜV vorgeführt, nur 7,9\% der Maschinen hatten so gravierende Mängel, die Plakette nicht erteilt wurde. Bei den Pkws waren dagegen nur 45,7 \% ohne Mängel, 18,4 \% hatten erhebliche Mängel.

Die häufigsten Mängel sind an der Beleuchtung zu finden (13,1 \%), an Achsen, Rädern und Reifen (7,6 \%) und am Fahrgestell (5,9\%). Dahinter verbergen sich in vielen Fällen defekte Glühlampen, nicht zugelassene Leuchten, falsche oder abgefahrene Reifen und ausgeschlagene Lager.

Als Grund für das offenbar stärkere Bemühen der Motorradfahrer um ihr Fahrzeug gibt der TüV das hohe Sicherheitsbewusstsein der Motorradfahrer an. Motorradfahrer seien keine Draufgänger, so Roger Eggers vom TÜV Nord, wer gerne das Gas aufdrehe, wolle sicher sein, dass seine Maschine in Ordnung ist und er kein Risiko eingeht. Ein weiterer Grund liege wohl darin, dass viele ihr Motorrad und das Motorradfahren als ein Hobby ansehen und die Besitzer ihrem teuren Freizeitgerät dementsprechend wesentlich mehr Aufmerksamkeit für Wartung und Pflege schenken.

Im Auftrag des Bundesministeriums für Verkehr, Bau- und Wohnungswesen hat die Bundesanstalt für Straßenwesen eine Studie zur Abschätzung möglicher Auswirkungen von Fahren mit Licht am Tag erarbeitet [Schönebeck 2005]. Dabei geht es nicht um die Lichteinschaltpflicht für Motorräder, sondern um ein allgemeines Tagfahrlicht für alle Kraftfahrzeuge. Nach den Ergebnissen dieser Untersuchung wird aus verschiedenen Gründen, unter Berücksichtigung unterschiedlicher technischer Lösungen und des Kosten-Nutzen-Verhältnisses, das Fahren mit Licht am Tag allgemein befürwortet. Allerdings stellt sich dabei dann die Frage nach der Sichtbarkeit von motorisierten Zweirädern. Für diese gilt seit 1988 in Deutschland die Pflicht, auch am Tag mit Abblendlicht zu fahren, da die schmale Silhouette des Einspurfahrzeugs zu Problemen bei der Erkennung und Einschätzung durch andere Verkehrsteilnehmer führt. Eingeschaltetes Abblendlicht verbessert die Erkennbarkeit motorisierter Zweiräder. Dem Problem der Sichtbarkeit von motorisierten Zweirädern begegnet die Bast in der vorliegenden Studie zunächst mit der Empfehlung, zur Unterscheidbarkeit der Motorradfahrer von mehrspurigen Kraftfahrzeugen bei der Einführung einer Pflicht für das Fahren mit Licht am Tag für motorisierte Zweiräder eine andere als die bisher vorgeschriebene Signalisierungsart (Abblendlicht) vorzusehen. Die BASt kündigt zugleich an, hierzu in naher Zukunft ein Projekt zu initiieren, dessen Ziel ein eindeutiger Vorschlag für die Signalisierung motorisierter Zweiräder am Tag sein soll, unter Berücksichtigung von Aspekten wie eindeutige Zuordnung des Signalbildes, technische Machbarkeit, Nachrüstung des Bestandes und Energieverbrauch.

[Unger 2004] konnte nachweisen, dass durch eine Anhebung des Kontrastes zwischen Motorradfahrer und Umgebung mit heller, tageslichtreflektierender Bekleidung und/oder lichttechnische Einrichtungen am Fahrzeug der Motorradfahrer in seiner Geschwindigkeit und Entfernung besser eingeschätzt werden kann. Aufgrund von Wahrnehmungsschwierigkeiten bei der Betrachtung der schmalen Silhouette des Motorradfahrers beim Herannahen, an z. B. eine Kreuzung, kommt es zu Schätzfehlern. Dabei wird die Geschwindigkeit mit steigendem Wert zunehmend unterschätzt und die Entfernung dementsprechend überschätzt (Abbildung 3-3 (nach [Grimm 1988] und [Leutzbach 1988])).

An die Problematik des Sehens und Gesehenwerdens von Motorradfahrern anknüpfend präsentierte die Firma Philips kürzlich rechtzeitig zum Beginn der Motorradsaison 2007 eine neuartige Motorrad-Scheinwerferlampe na- 
mens MotoVision (n-tv, Sendung vom 14.04.2007, (www. burning-out.de, 21.04.2007). Diese verbessert durch eine neue Technologie gleichzeitig die Sicht für den Motorradfahrer und die Sichtbarkeit durch andere Verkehrsteilnehmer. Durch die orangefarbene Reflektion von MotoVision heben sich Motorräder deutlicher von übrigen Verkehrsteilnehmern ab und werden besser wahrgenommen, insbesondere da ein generelles Fahren mit Licht am Tag unter anderem von der BASt, wie oben bereits dargestellt, empfohlen wird und immer mehr Pkw-Fahrer bereits freiwillig auch am Tag mit eingeschaltetem Licht fahren. Die Sicht für den Motorradfahrer selbst, vor allem nachts, soll dadurch verbessert werden, dass die neue Lampe laut Philips $40 \%$ mehr weißes Licht erzeugt und somit einen 10 bis $20 \mathrm{~m}$ längeren Lichtkegel aufweist.

Zum besseren Sehen gehören aber nicht nur gute Lichtverhältnisse, sondern auch eine ausreichende Erfassung der Umgebung durch den Fahrer. Dazu präsentierte die Bast im Jahre 2001 eine Studie über Anforderungen an Rückspiegel von Krafträdern [van de Sand 2001]. Demnach sind die Fahrerplätze von Krafträdern meist nur unzureichend ergonomisch gestaltet. Beispielsweise gibt es bei den meisten Motorrädern keine Möglichkeit zur Anpassung des Sitzes an unterschiedliche Körpermaße der Fahrer. Auch Spiegelanordnung und rückwärtiges Sichtfeld wurden bisher kaum oder nur unzureichend analysiert. Durch die Studie sollte daher die Lücke geschlossen werden, welche bisher in einer fehlenden Datenbasis für Krafträder zur geplanten Überarbeitung einer Richtlinie zur Angleichung der Rechtsvorschriften der EU-Mitgliedsstaaten über die indirekte Sicht aus Kraftfahrzeugen bestand. In dieser Untersuchung wurde zunächst eine Literaturrecherche zu aktuellen nationalen und europäischen Gesetzgebungen sowie zum Stand der Technik bei Spiegelanordnungen durchgeführt.

In einem weiteren Arbeitsschritt wurden die seitlichen und rückwärtigen Sichtfelder von jeweils zwei Tourenkrafträdern, Sport- und Rennsportmaschinen, Geländekrafträdern/Enduros, Rollern und Choppern untersucht. Zur Abschätzung der Abhängigkeit des rückwärtigen Sichtfeldes von der Fahrerstatur wurden die Untersuchungen nacheinander mit zwei verschieden großen Fahrern durchgeführt, und zwar einer $5 \%^{1}$ Frau und einem 95 \% Mann. Zur besseren Vergleichbarkeit erhielten die

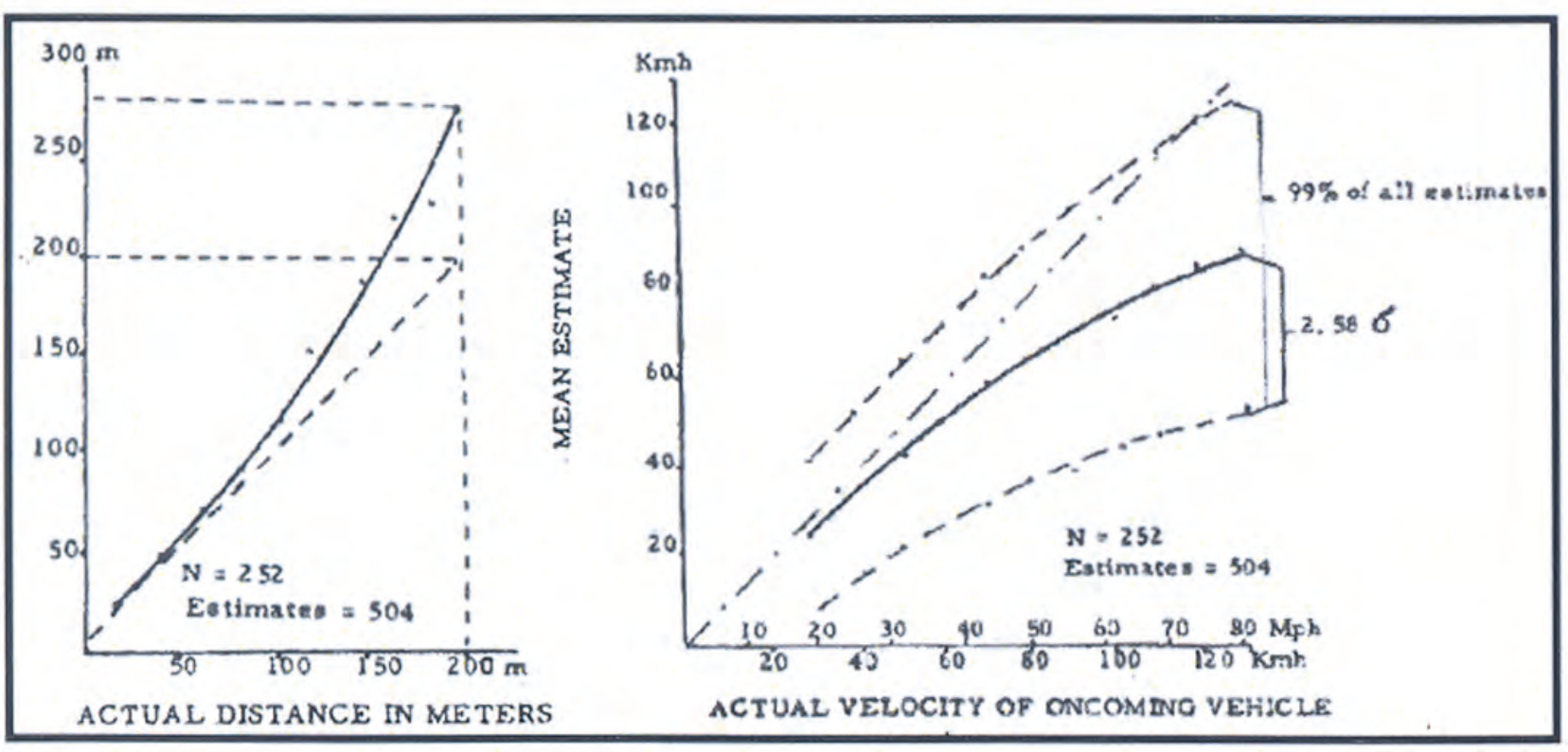

Abbildung 3-3: Schätzfehler bei der Wahrnehmung, Quelle: [Grimm 1988]

1 Als 5\% Frau wird diejenige Frau bezeichnet, deren Größe und Gewicht von nur 5\% Frauenanteil der Bevölkerung unterschritten wird. Äquivalent verhält es sich mit dem 95\% Mann. Nur 5\% der Männer sind schwerer und größer als diese Person. 
Versuchspersonen den gleichen Helmtyp und die typische Motorrad-Schutzkleidung. Die ermittelten äußeren Sichtwinkel lagen nahezu alle bei rund $20^{\circ}$. Dieser Bereich ist für eine umfassende Beobachtung des rückwärtigen Verkehrs allein über Spiegel nicht ausreichend, ein Schulterblick ist daher unverzichtbar. Eine solche Abwendung des Kopfes vom vorderen Bereich zur Seite erweist sich aber gerade bei hohen Geschwindigkeiten aufgrund der Beeinträchtigung der Fahrstabilität und der Fahrzeugbeherrschung als hohes Gefahrenpotenzial.

In einem weiteren Schritt wurde eine Lasermesspuppe entwickelt, mit der es möglich war, die rückwärtigen Sichtverhältnisse anhand einer eigens entwickelten, reproduzierbaren Prüfmethode zu untersuchen. An einem variablen Versuchsträger wurden dann verschiedene Spiegelpositionen und -arten untersucht, und zwar zum einen mit der Lasermesspuppe, zum anderen mit den Fahrern für eine objektive Vergleichsmöglichkeit. Aufgrund dieser Ergebnisse wurden Verbesserungsvorschläge zur Spiegelanordnung und Mindestanforderungen an zukünftige Spiegelauslegungen formuliert. Aus den Ergebnissen der subjektiven und objektiven Bewertung ergeben sich folgende Erkenntnisse:

- Vergrößerung des Sichtfeldes, etwa durch asphärische Spiegel, jedoch müssen gesetzliche Werte bereits durch plane Teil erfüllt werden

- Vermeidung des Einflusses von Vibrationen, dazu Befestigung der Spiegel an der Verkleidung empfehlenswert

- sinnvolle Spiegelanordnung unterhalb der Lenker verhindert Sichtverdeckungen durch Fahrerextremitäten, wobei jedoch keine Einschränkungen durch den Beifahrer auftreten dürfen

- Einsatz rechteckiger Spiegel mit ausgewogenem Höhen-Breiten-Verhältnis. Vermeidung runder Spiegel bzw. Forderung nach einer einschreibbaren Mindestfläche

- ausreichend große Spiegelfläche, in jedem Fall größer $100 \mathrm{~cm}^{2}$

- gute Abbildungsqualität auch im Spiegelrandbereich

- zur genauen Positionierung feinfühlige Einstellbarkeit erforderlich, die sich im Fahrbetrieb nicht ändern darf.
Eine Schwäche der aktuellen EG-Richtlinie ist, dass das vorgeschriebene rückwärtige Sichtfeld durch eine beliebige Spiegelposition erreicht werden kann, die nicht unbedingt einer sinnvollen Einstellung entsprechen muss. Bei dem Lasermessverfahren müssen die Spiegel im Gegensatz zur Richtlinie vorher auf eine Grundposition justiert werden. Dieses Verfahren stellt somit weitaus höhere Anforderungen an die rückwärtigen Sichtverhältnisse.

Mit der Lasermesspuppe wurden auch asphärische Spiegel untersucht. Dabei zeigte sich, dass sich mit asphärischen Spiegeln Verbesserungen des äußeren Sichtwinkels bis über $25^{\circ}$ erreichen lassen. Subjektive Untersuchungen ergaben jedoch ein Eingewöhnungsbedürfnis. Zudem ist die Abbildungsqualität durch Verzerrungen teilweise beeinträchtigt. Im Stadtverkehr wurde subjektiv keine Verbesserung durch asphärische Spiegel festgestellt. Daher erscheint eine Zweiteilung des Sichtfeldes in einen Mindestbereich bei Verwendung sphärischer Spiegel und einen deutlich größeren Bereich bei Verwendung asphärischer Spiegel nicht sinnvoll. Für das Einsatzpotenzial asphärischer Spiegel ergab sich weiterer Forschungsbedarf.

Aus den Ergebnissen der subjektiven und objektiven Untersuchungen und dem hierfür entwickelten Prüfverfahren mittels Lasermesspuppe wurden Vorschläge zur Überarbeitung der aktuellen Gesetzeslage abgeleitet. 


\section{$4 \quad$ Entwicklung des Unfallgeschehens}

Für aktuelle Aussagen zum Unfallgeschehen von motorisierten Zweiradbenutzern sind aus der Statistik Zahlen für den 15 Jahre - Zeitraum 1991-2005 herausgesucht und ausgewertet worden. Vergleiche der einzelnen Bundesländer mit der Bundesstatistik können größtenteils mangels Datenverfügbarkeit nicht für dieses Zeitintervall durchgeführt werden. In diesen Fällen wird die Betrachtung auf den 10 Jahre - Zeitraum von 1996 bis 2005 konzentriert. Die Zeitreihen zu der Getöteten- und Verunglücktenentwicklung im Bundesgebiet wurde für das Jahr 2006 aktualisiert. Fahrleistungs- und Bestandsdaten mit aktuellen Zahlen für 2006 erscheinen Anfang 2008 (Verkehr in Zahlen 2007/2008) und konnten für den Schlussbericht nicht mehr berücksichtigt werden.

Die Auswertungen bauen auf den vorhandenen Untersuchungen von [Maier 2000], [Assing 2002] und [Koch 2006] auf. Die vorliegende Untersuchung betrachtet hautsächlich das Unfallgeschehen in den drei Jahren von 2004 bis 2006. In den o. g. drei Literaturangaben ist dieser Zeitbereich nicht vollständig ausgewertet worden. Die nachfolgenden Statistiken sind als Aktualisierung des Unfallgeschehens mit Motorradfahrern zu werten.

\subsection{Generelle Entwicklungen}

\subsubsection{Fahrzeugbestand}

Für die Jahre 1991 bis 1993 sind in den Statistiken zum Kraftfahrzeugbestand nur bedingt Zahlen zu finden. Verkehr in Zahlen 2006/2007 [BMVBS 2006] stützt sich dabei auf das Deutsche Institut für Wirtschaftsforschung (DIW), die für die neuen Bundesländer Bestandsberechnungen durchgeführt haben. Die Bestandentwicklung bei Motorrädern, Mofa/Mopeds und Personenkraftwagen ist in Abbildung 4-1 dargestellt.

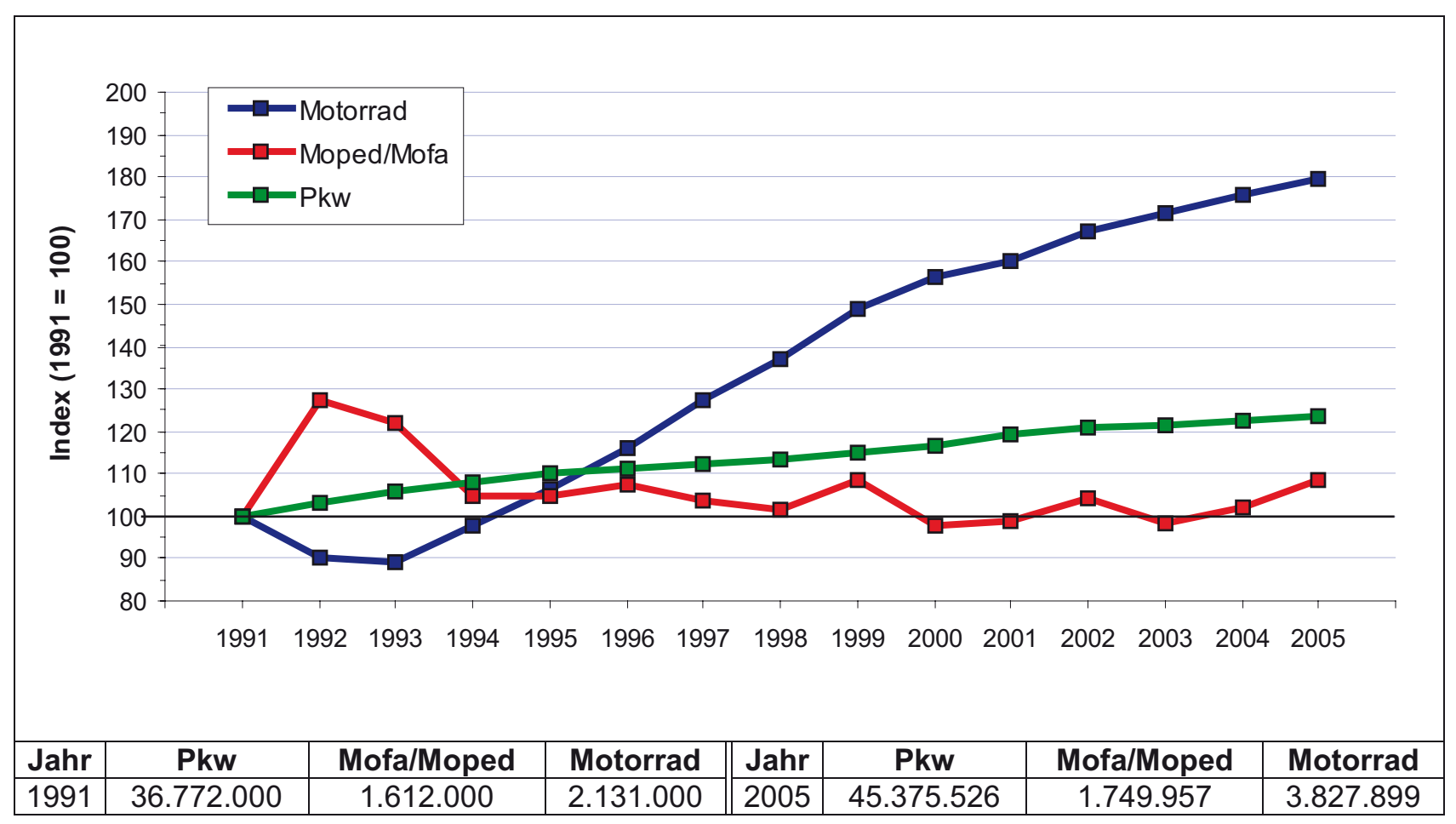

Abbildung 4-1: Entwicklung des Fahrzeugbestandes von motorisierten Fahrzeugen im Vergleich zu den Personenkraftwagen nach [BMVBS 2006] 
Für das gesamte Bundesgebiet gibt die Statistik für das Jahr 1991 ein Bestand an 44,9 Mio. Kraftfahrzeugen an. Darin enthalten sind rund 1,6 Mio. Mofa/Mopeds (4\%) und 1,5 Mio. Motorräder (3\%). Der Anteil an Personenkraftwagen lag bei $82 \%$ (36,8 Mio.). Der Pkw-Bestand nahm bis zum Jahr 2005 um 8,6 Mio. Fahrzeuge (+ $23 \%)$ zu. Eine eindeutige Zu- oder Abnahme ist bei der Anzahl der Mofa/Mopeds nicht gegeben. Die Bestandszahlen bewegten sich in den letzten 15 Jahren zwischen dem Ausgangswert von 1991 und einem um 10 \% höheren Wert. Bei den Motorrädern ist ein deutlicher Anstieg seit 1993 zu verzeichnen. Der Bestand an Motorrädern hat sich fast verdoppelt und liegt jetzt bei 3,8 Mio. Fahrzeugen (+ $80 \%$ ). Bezogen auf den Gesamtbestand von 53,8 Mio. Fahrzeugen erhöhte sich der Anteil der Motorräder auf $7 \%$. Die Zunahme im Bestand könnte damit begründet werden, dass die Motorräder als Zweitfahrzeuge für die Freizeit einen hohen Zuspruch gefunden hat. Diese Annahme wird durch die Bestandsentwicklung nach Altersgruppen von [Assing 2002] unterstützt, der zeigte, dass der Anstieg ausschließlich auf die Altersgruppe „35 und mehr“ zurückzuführen ist. Die Entwicklung in dieser Altergruppe zeigte einen Anstieg um 170 \% für den Zeitraum von 1994 bis 2000.

\subsubsection{Fahrleistungen von Kraftfahrzeugen}

In der amtlichen Statistik des Bundes ist keine fahrzeugartenbezogene Fahrleistung aufgeführt. Lediglich für den Gesamtverkehr sind Fahrleistungen für alle Straßen und separat nur für Autobahnen aufgeführt. In [BMVBS 2006] sind jährliche Fahrleistungen differenziert nach sieben

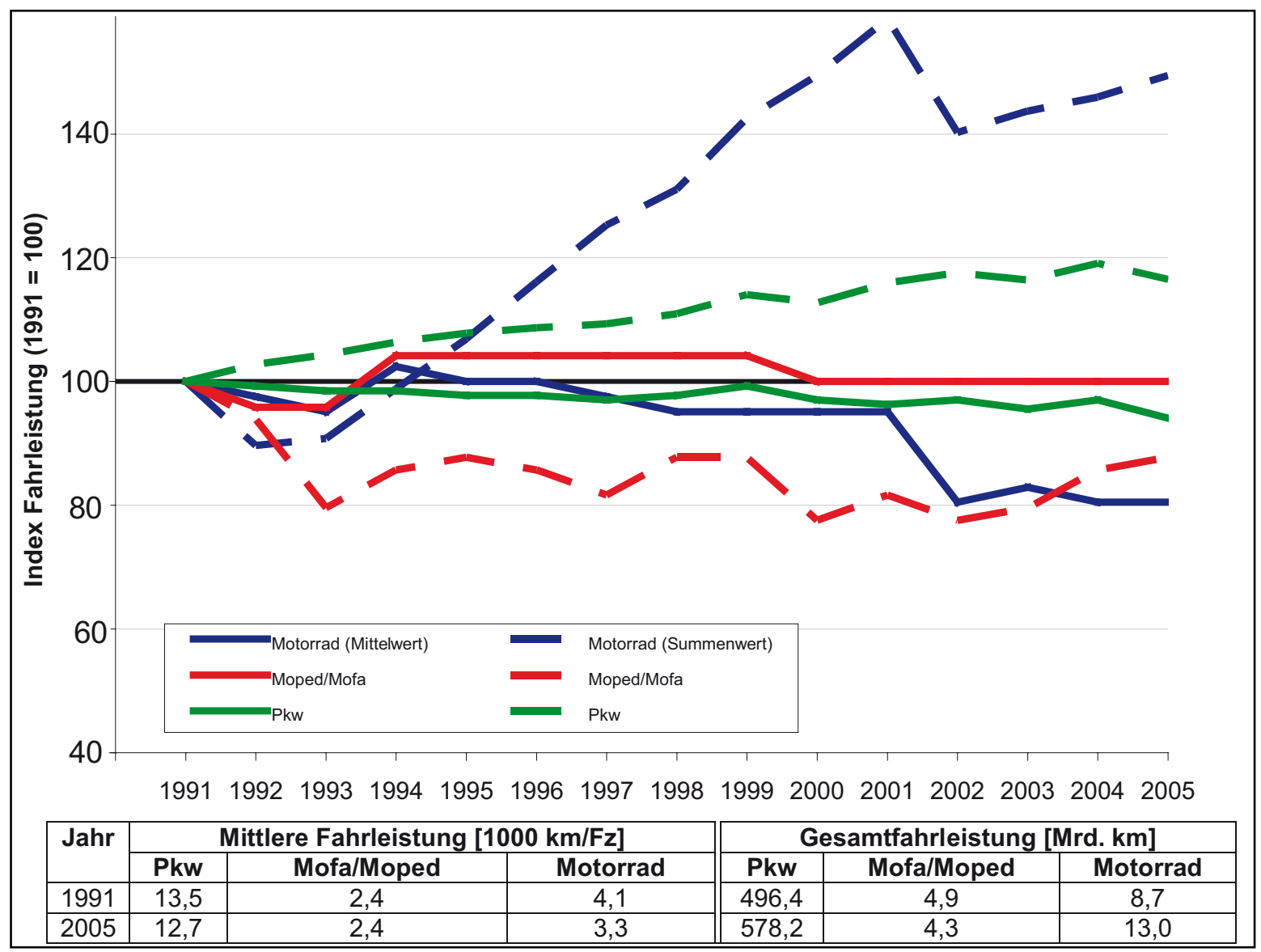

Abbildung 4-2: Entwicklung der Fahrleistungen von motorisierten Fahrzeugen im Vergleich zu den Personenkraftwagen nach [BMVBS 2006] 
Fahrzeugarten angegeben. Diese Werte sind auf Basis von Modellrechnungen vom DIW Berlin aus den Kennwerten Fahrzeugbestand, durchschnittlicher Verbrauch und durchschnittliche Fahrleistung ermittelt worden. Die Entwicklung der mittleren Fahrleistung eines Fahrzeuges und die Gesamtfahrleistung der Verkehrsarten zeigt Abbildung 4-2.

Die Gesamtfahrleistung der Motorräder und Pkw nahmen von 1991 an kontinuierlich zu. Dieser Anstieg wird mit der Erhöhung der Bestandszahlen begründet, da die mittlere Fahrleistung eines einzelnen Fahrzeuges über die 15 Jahre nahezu konstant blieb (bei den Motorrädern bis 2001). Dieser Zusammenhang zeigt sich auch bei den Mopeds/Mofas. Der Rückgang der mittleren Fahrleistung zwischen dem Ausgangsjahr 1991 und 1993 kann auf die Eingliederung der Fahrzeuge, die in diesem Zeitbereich in den neuen Bundesländern zugelassen waren, zurückgeführt werden. Zwischen den beiden deutschen Gebieten gab es unterschiedliche Klassengrenzen. In den Statistiken werden diese drei Jahre häufig nicht mit Werten ausgefüllt. Die in den Abbildungen Abbildung 4-1 und Abbildung 4-2 angegebenen Zahlen sind vom DIW berechnet worden.

Der Anstieg der Gesamtfahrleistung der Motorräder wird zwischen 2001 und 2002 unterbrochen. Dieser kurzzeitige Rückgang wird von [Koch 2006] damit begründet, dass die Fahrleistungen auf genaueren Erhebungen der BASt von 1993 basieren und jeweils mit dem aktuellen Bestand der folgenden Jahre multipliziert worden. Neue

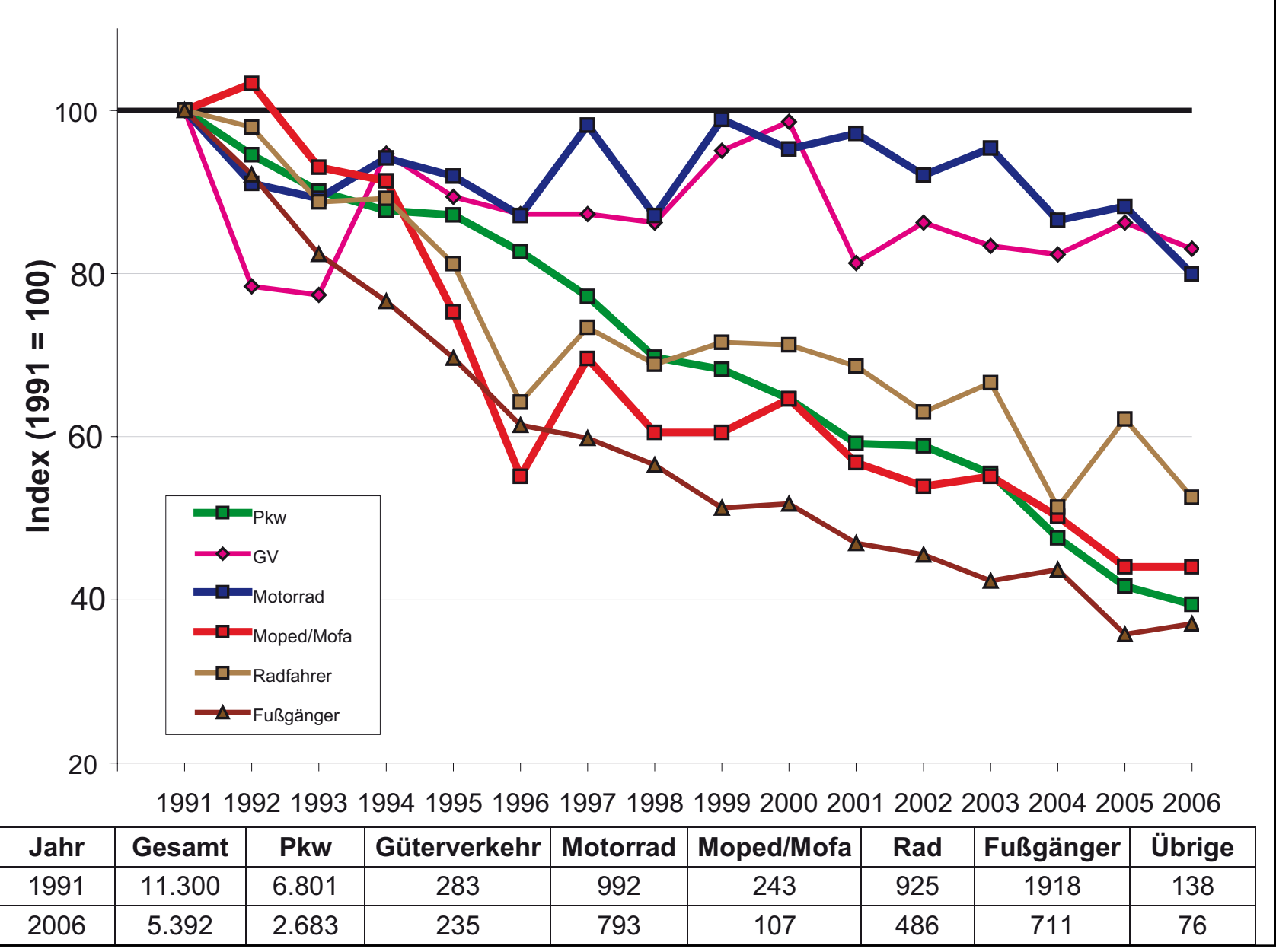

Abbildung 4-3: Getötetenentwicklung in Deutschland getrennt nach der Art der Beteiligung für den Zeitraum von 1991 bis 2006 nach [Destatis 2007] 
Fahrleistungserhebungen im Jahr 2002 zeigten, dass diese bestandsabhängige Entwicklung in dieser Größenordnung nicht eingetreten ist, sondern im Laufe der Zeit kontinuierlich gesunken ist. Daher sind für das Jahr 2002 Korrekturen vorgenommen worden. Der Anstieg bis 2005 fällt somit geringer aus als noch zwischen 1993 und 2001 angenommen worden ist.

\subsubsection{Anzahl und Schwere der Unfälle}

Im Bundesgebiet sank die Zahl der getöteten Verkehrsteilnehmer von $11.300 \mathrm{im}$ Jahr 1991 auf $5.091 \mathrm{im} \mathrm{Jahr}$ 2006, was einem Rückgang von 55 \% entspricht. Dieser Trend wird ausschließlich durch die Verringerung der Getötetenzahlen bei Pkw-, Moped-/Mofabenutzern, Radfahrern und Fußgängern bestimmt (Abbildung 4-3).
Dagegen sank die Zahl der getöteten Motorradbenutzer nur zwischen 1991 und 1993 um rund 10 \%. In den Folgejahren bewegte sich der Anteil zwischen 90 und $100 \%$. Ein leichter rückläufiger Trend scheint sich seit 2000 eingestellt zu haben. Lag der Anteil zum Ausgangjahr 1991 im Jahr 1999 noch bei 99 \%, ergab sich 2006 nur noch ein Anteil von $80 \%$. Vergleichend dazu sank die Zahl der getöteten Moped/Mofabenutzer zwischen 1991 und 2006 von 243 auf 107 um $44 \%$.

Im Vergleich zu der Getötetenentwicklung weist die Entwicklung der Verunglückten teilweise andere Tendenzen auf (Abbildung 4-4).

Bezogen auf die Anzahl aller Verunglückten im Straßenverkehr ergab sich ein Rückgang um 17 \%. Der Rückgang

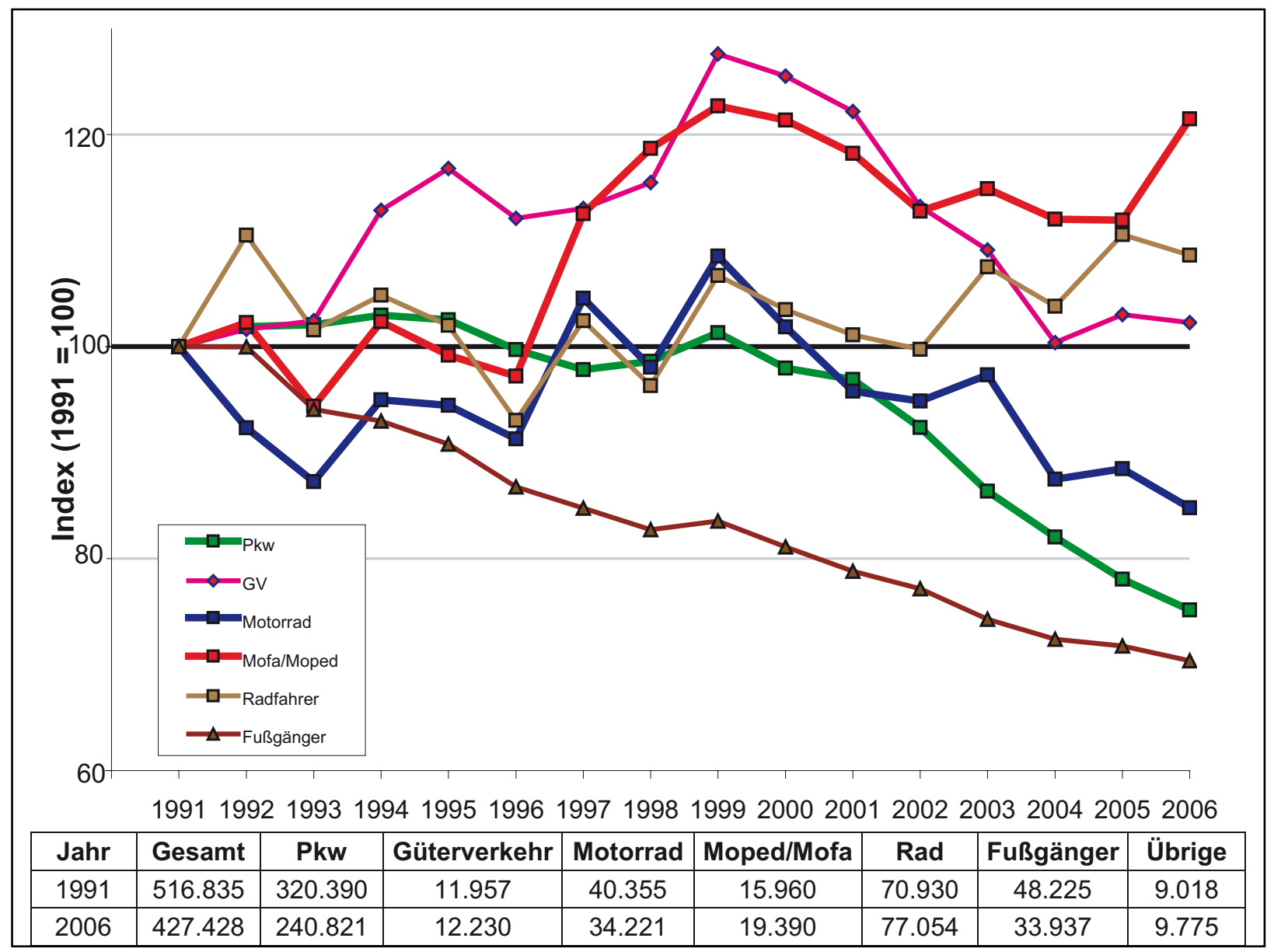

Abbildung 4-4: Verunglücktenentwicklung in Deutschland getrennt nach der Art der Beteiligung für den Zeitraum von 1991 bis 2006 nach [Destatis 2007] 
wird wieder durch die Abnahme der Verunglücktenzahlen der Pkw-Benutzer und der Fußgänger beschrieben. Bei den Motorradbenutzern zeigt sich ein ähnlicher Trend wie bei der Entwicklung der Getötetenzahlen. Nach einem Rückgang zwischen 1991 und 1993 nahm die Anzahl der Verunglückten bis 1999 stetig zu. Seit 2000 ist wieder ein Rückgang zu verzeichnen. Die Entwicklung der verunglückten Moped/Mofabenutzer dagegen ergab für die ersten Jahre keine Änderung. Von 1997 stieg die Zahl der Verunglückten auf 123 \% (1999). In den Folgejahren ist bis 2005 wieder ein rückläufiger Trend zu verzeichnen. Dieser wurde aber im Jahr 2006 mit dem stärksten Anstieg seit 1996 unterbrochen. Mit Ausnahme der Moped/ Mofas und Radfahrer zeigt sich generell für alle Verkehrsteilnehmergruppen eine Abnahme der Verunglücktenzahlen seit dem Jahr 2000.

Da sich Unfälle unterschiedlicher Schwere nicht gleichartig entwickeln, bietet sich eine Gesamtbetrachtung von Unfallzahl und Unfallschwere durch die Verwendung volkswirtschaftlicher Verluste durch Straßenverkehrsunfälle an, abgekürzt als Unfallkosten bezeichnet. Dazu sind aus der Anzahl der Unfälle mit Personenschaden U(P) und der Verunglücktenzahlen sind für die motorisierten Zweiradfahrer und die übrigen Verkehrsteilnehmer direkt angepasste Unfallkostensätze berechnet worden. Die Entwicklung von 1996 bis 2005 zeigt Abbildung 4-5.

Waren die Unfälle im Jahr 1996 mit rund 70.000 Euro pro $U(P)$ nahezu gleich schwer gewesen, ergeben sich 2005 deutliche Unterschiede. Zwar ist für beide Kollektive eine Verringerung der Unfallschwere festzustellen, die Reduzierung fällt jedoch bei den übrigen Verkehrsteilnehmern mit einem Rückgang auf ca. 52.000 Euro pro $U(P)$ deutlich aus, während der Wert bei den motorisierten Zweiradfahrern nur leicht auf 65.000 Euro je $U$ $(P)$ zurückgeht. Damit weist ein $U(P)$, bei welchem ein motorisierter Zweiradfahrer beteiligt ist, im Mittel etwa 25 \% höhere Unfallkosten auf als ein U (P) ohne Beteiligung von motorisierten Zweirädern. Werden die Entwicklungen getrennt nach Ortslage untersucht, ist zu erkennen, dass die entstandene Diskrepanz vor allem aus den Entwicklungen im Außerortsbereich resultiert. Im Fall der motorisierten Zweiradfahrer ist auf Autobahnen eine Zunahme der mittleren Unfallschwere von 110.000 auf 126.000 Euro pro $U(P)$ festzustellen, während der Wert innerorts und auf Landstraßen stagniert bzw. leicht zurückgegangen ist. Bei den übrigen Verkehrsteilnehmern ist vor allem im Bereich der Landstraßen sowie auf Autobahnen ein deutlicher Rückgang zu verzeichnen.

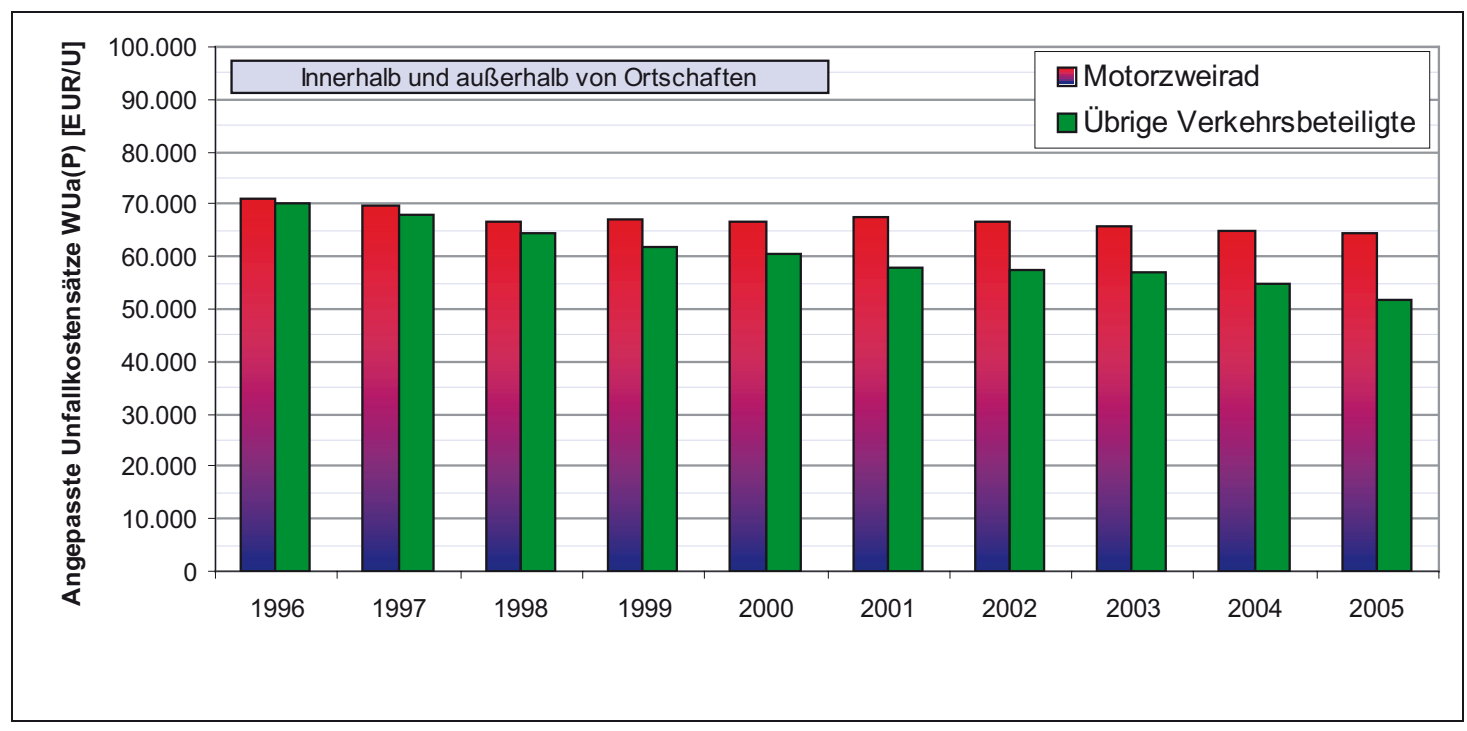

Abbildung 4-5: Entwicklung der direkt angepassten Unfallschwere für Unfälle mit Personenschaden in Deutschland für motorisierte Zweiradbenutzer und alle übrigen Verkehrsteilnehmergruppen für den Zeitraum von 1996 bis 2005 
Die Entwicklung der Struktur nach Ortslage der bei einem Unfall getöteten Motorradfahrer ergibt von 1991 bis 2005 das in Abbildung 4-6 dargestellte Bild.

Für das Jahr 2005 kann entnommen werden, dass etwa 2/3 aller getöteten Motorradfahrer auf Landstraßen verunglücken. Auf innerörtliche Straßen fallen rund $25 \%$ der Getöteten, auf Autobahnen weniger als $10 \%$. Die Entwicklung seit 1991 zeigt gegenüber dieser Verteilung keine deutlichen Abweichungen.

Die zusätzliche Analyse der Verunglücktenstruktur nach Ortslage (Abbildung 4-7) zeigt, dass nahezu 2/3 aller Motorradfahrer innerorts verunfallen. Auf Autobahnen entfällt ein kleiner Teil von weniger als $5 \%$, die übrigen Motorradfahrer verunglücken auf Landstraßen. Auch hier sind aus der Entwicklung seit 1991 keine wesentlichen Veränderungen zu erkennen.

Die vorangegangenen Betrachtungen verdeutlichen, dass vor allem innerorts sowie im Landstraßenbereich Handlungsbedarf besteht, die Verkehrssicherheit für motorisierte Zweiradfahrer zu verbessern und an die positive Entwicklung der übrigen Verkehrsteilnehmer anzupassen.

\subsubsection{Raten - Maß für die relative Unfallgefahr}

Die fahrleistungsbezogene Wahrscheinlichkeit, in einen Unfall verwickelt zu werden, beschreibt die Unfallrate (auch als Unfallgefahr bezeichnet). Sie entspricht der durchschnittlichen Anzahl der Unfälle, die auf eine bestimmte Fahrleistung z. B. $1 \mathrm{Mrd}$. $\mathrm{Kfz}^{*} \mathrm{~km}$ entfallen. Die Entwicklung der Getötetenrate ist in Abbildung 4-8 dargestellt.

Im Jahr 1991 starben in Deutschland auf 1 Mrd. Kfz* $\mathrm{km}$ 114 Motorradbenutzer, im Jahre 2005 waren es noch 67. Die Gefahr, bei einem Unfall getötet zu werden, ist seit 1991 bei den Motorradfahrern um ca. 40 \% und bei den Moped-/Mofafahrern um rund 50 \% zurückgegangen, jedoch bei weitem nicht so deutlich wie die Rate für alle übrigen Kfz. Vergleichsweise liegt die absolute Größe dieser Unfallgefahr bei den motorisierten Zweirädern insbesondere für die Motorradbenutzer sehr hoch. Das Verhältnis der Getötetenraten - dargestellt in Abbildung 4-9 - verdeutlicht die Unterschiede zwischen Motorradfahrern bzw. Moped-/Mofafahrern und den übrigen KfzNutzern bezüglich der Gefahr durch einen Verkehrsunfall tödlich zu verunglücken.

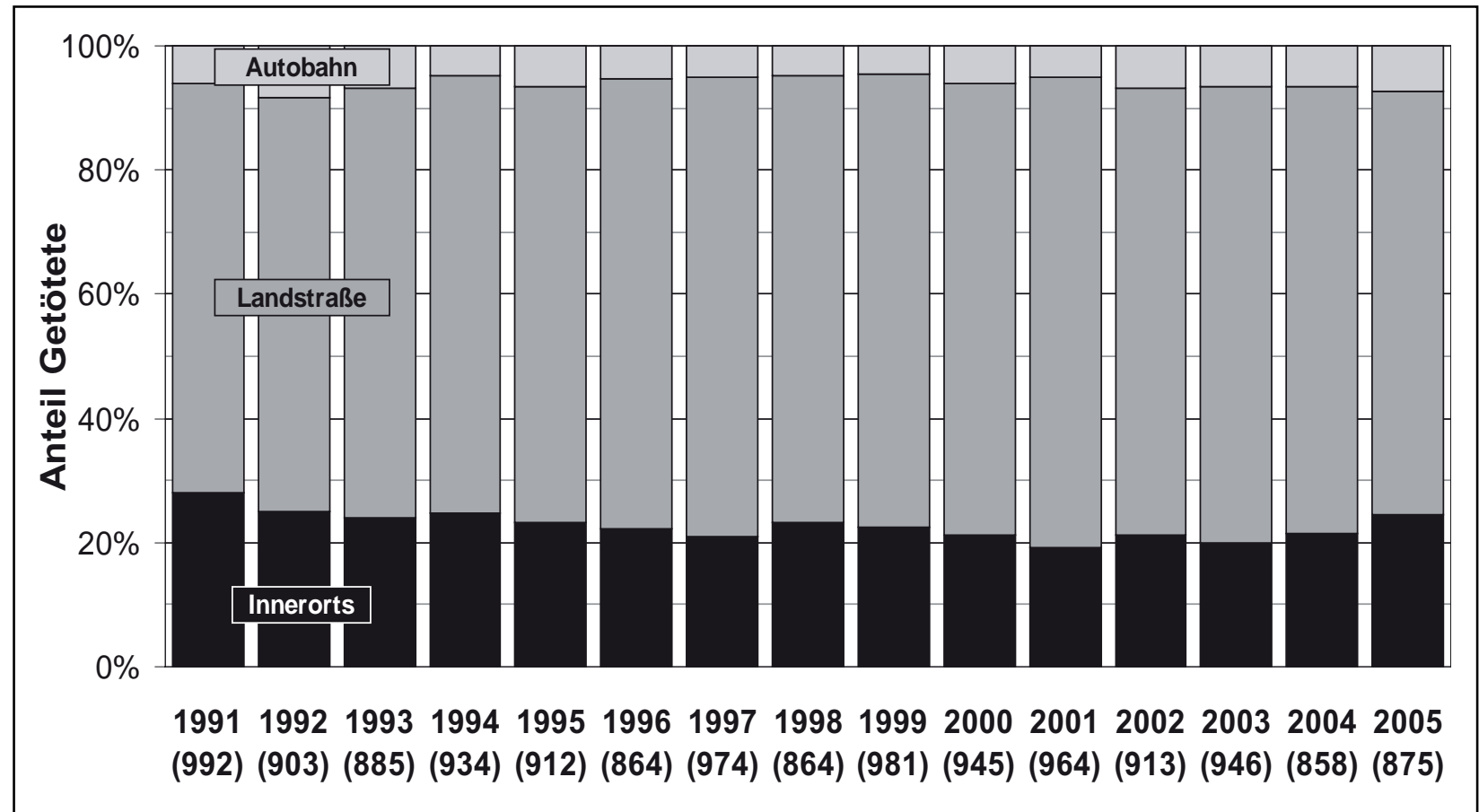

Abbildung 4-6: Entwicklung der Getötetenenstruktur nach Ortslage für getötete Motorradfahrer in Deutschland $1991-2005$ 


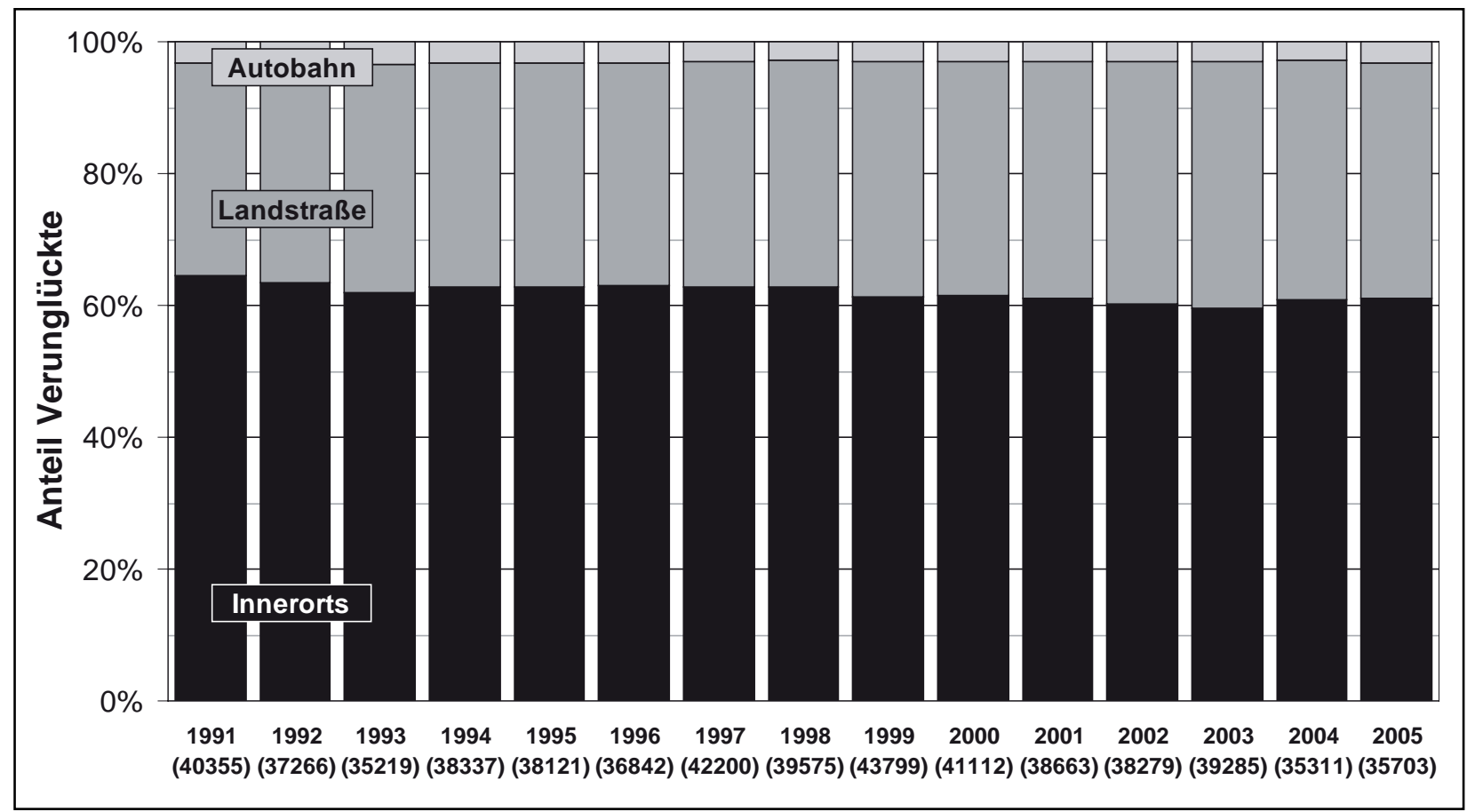

Abbildung 4-7: Entwicklung der Verunglücktenstruktur nach Ortslage für verunglückte Motorradfahrer in Deutschland 1991-2005

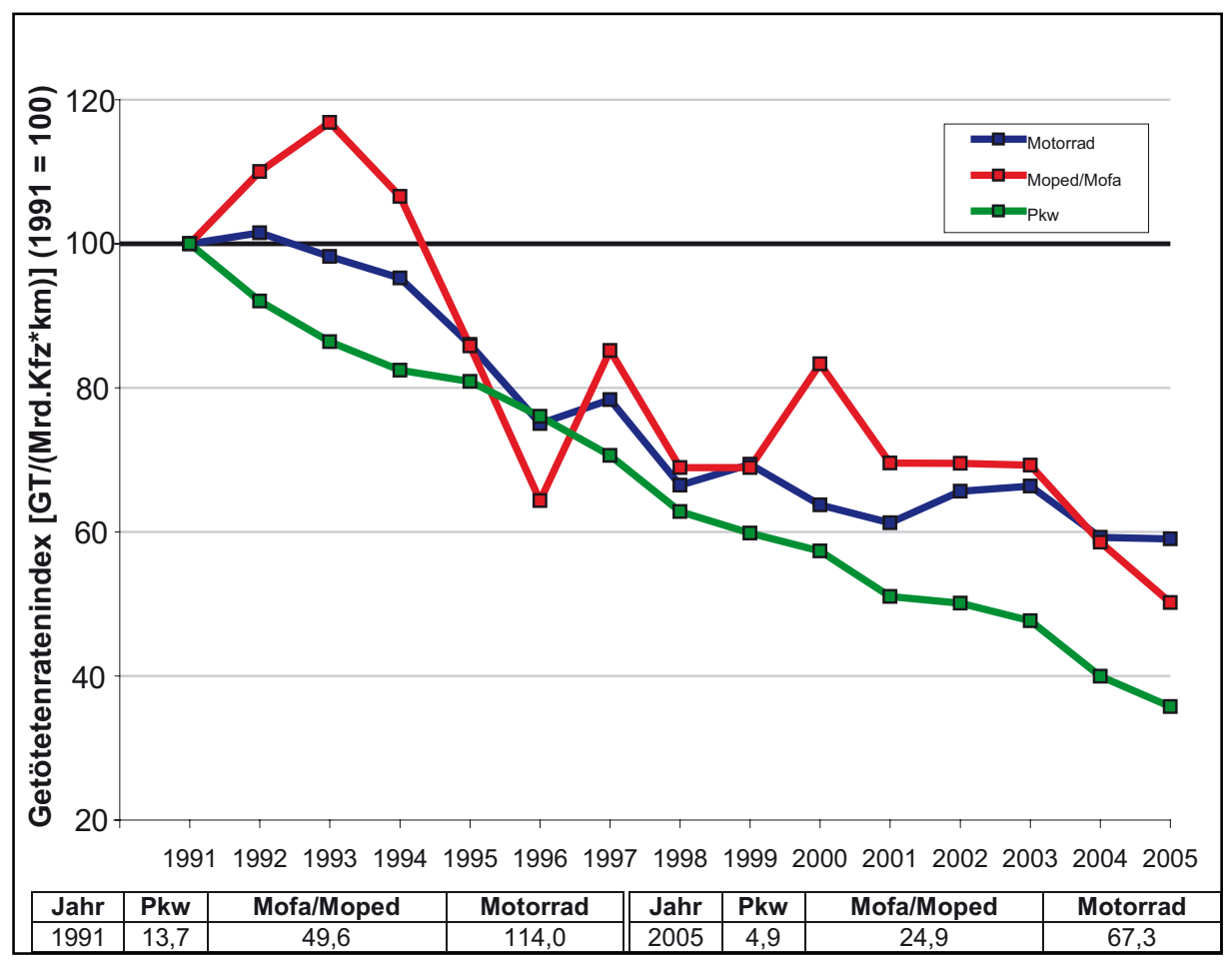

Abbildung 4-8: Entwicklung der Getötetenrate der Nutzer von Motorrädern und Moped/Mofas im Vergleich zu den Nutzern der übrigen Kraftfahrzeuge nach [Destatis 2007] und [BMVBS 2006] 
Lag für einen Motorradfahrer die Gefahr bei einem Unfall getötet zu werden bereits 1991 neunfach höher als für die übrigen Kfz-Nutzer, so ist dieses Verhältnis bis 2005 auf ein 14 fach höheres Risiko angestiegen. Bei der Betrachtung der Moped/Mofas ist der Unterschied nicht so deutlich ausgeprägt, aber mit einem über fünffach so hohen Wert im Jahr 2005 immer noch enorm. Die Entwicklung zeigt hier seit 1991 ein drei- bis sechsfaches Risiko im Vergleich zu den übrigen Kfz-Benutzern.

Diese Betrachtung zeigt deutlich die bereits erwähnte Notwendigkeit, die Entwicklung der motorisierten Zweiradfahrer hinsichtlich der Verkehrssicherheit an diejenige der übrigen Verkehrsteilnehmer anzugleichen.

\subsection{Unfallgeschehen der Zielgruppen}

Für bestimmte Gruppen motorisierter Zweiradfahrer, die möglicherweise eine Zielgruppe für gezielte Maßnahmenansätze darstellen können, wurde über eine Sonderabfrage beim Statistischen Bundesamt die Unfallzahlen der Jahre 2004 und 2005 differenziert nach den Merkmalen Ortslage, Straßenzustand und Lichtverhältnisse erworben und ausgewertet. Dabei wurden ausschließlich die Unfälle mit Personenschaden berücksichtigt.

Zum Zeitpunkt der Abfrage stand die in Abschnitt 1.3 erläuterte Definition der Zielgruppen noch nicht fest, so dass die abgefragten Gruppen nicht exakt mit den spä-

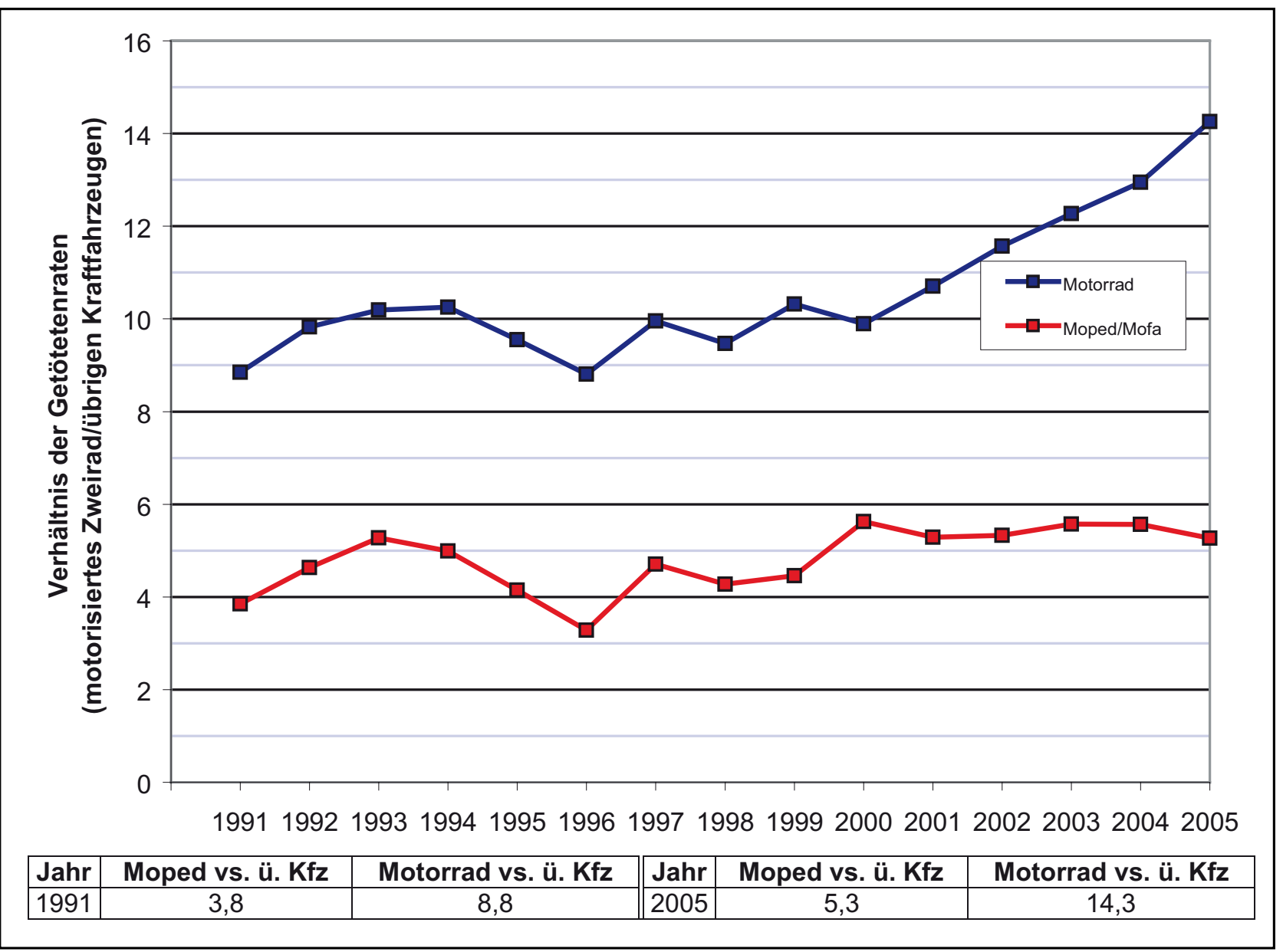

Abbildung 4-9: Entwicklung des Verhältnisses der Getötetenrate von Motorrädern bzw. Moped/Mofas zu den übrigen Kraftfahrzeugen von 1991 bis 2005 nach [Destatis 2007] und [BMVBS 2006] 
ter definierten Zielgruppen übereinstimmen. Die Zahlen ermöglichen dennoch auf bundesweiter Datenbasis die Überprüfung spezieller Gruppen auf Auffälligkeiten im Unfallgeschehen, insbesondere in Bezug auf die genannten Unfallmerkmale. Eine Entwicklung kann dabei für die zwei zur Verfügung stehenden Jahre analysiert werden.

Die Kriterien für die Festlegung der abgefragten Zielgruppen sind in Tabelle 4-1 dargestellt.

Die im Vergleich zur Zielgruppendefinition in Abschnitt 1.3 weitere Abgrenzung der Zielgruppen z. B. anhand der Unfallzeit (werktags vs. Wochenende, bestimmte Tagesstunden) kann schärfere Ergebnisse für die Zielgruppen ermöglichen und führt aufgrund der bundesweiten Datengrundlage nicht zu sehr kleinen Fallzahlen. Generell werden für alle Zielgruppen nur die Unfälle der Monate April bis Oktober verwendet, um die Besonderheiten des Winters auszuschließen. Dies erscheint gerechtfertigt, da lediglich Unfälle mit Mofafahrern und Schülern nennenswert auch im Winter auftreten.

Abbildung 4-10 zeigt für die Jahre 2004 und 2005 zusammengefasst die Aufteilung der Unfälle jeder Zielgruppe auf die Ortslagen innerorts, außerorts (ohne BAB) und Autobahnen. Als Vergleichswert dient die blaue Säule mit allen $U(P)$ mit Beteiligung motorisierter Zweiräder in den beiden Jahren. Insgesamt zeigt sich, dass mit den so definierten Zielgruppen in etwa die Hälfte dieser Unfälle abgedeckt ist.
Innerorts fallen neben den Fahranfängern vor allem die Jugendlichen mit einem überdurchschnittlichen Unfallanteil auf. Die Berufsfahrer und insbesondere die Frauen liegen unter dem mittleren Anteil. Bei den Berufsfahrern ist allerdings zu berücksichtigen, dass vor allem diese Gruppe innerorts auch in den Wintermonaten auf ihr motorisiertes Zweirad zurückgreifen wird, diese Unfälle aufgrund des generellen Ausschlusses der Wintermonate November bis März aber nicht der Zielgruppe zugeordnet sind.

Auf den Landstraßen zeigt sich ein erhöhter Anteil an Unfällen für die Gruppe der Frauen ab 21. Auf Autobahnen liegen sehr geringe Absolutzahlen zugrunde, so dass diese Betrachtung nicht interpretiert werden kann. Die Zahlen verdeutlichen jedoch, dass nur ein sehr geringer

Anteil aller Unfälle (2\%) auf Autobahnen passiert und der vordringliche Handlungsbedarf innerorts und auf Landstraßen zu sehen ist.

Weiterhin wurden die relativen Veränderungen der Unfälle einer Zielgruppe in der Entwicklung von 2004 zu 2005 differenziert nach Ortslage, Straßenzustand und Lichtverhältnissen untersucht. Der Trend für alle $U(P)$ mit motorisierter Zweiradbeteiligung ist zum Vergleich als blaue Säule abgebildet (Abbildung 4-11).

Bis auf die Gruppe der Jugendlichen ist für alle Zielgruppen ein Anstieg der Unfallzahlen zwischen $4 \%$ und $9 \%$

\begin{tabular}{|l|c|c|c|c|c|}
\hline & $\begin{array}{c}\text { Gruppe 1 } \\
\text { Beruftskraftfahrer }\end{array}$ & $\begin{array}{c}\text { Gruppe 2 } \\
\text { Fahranfänger }\end{array}$ & $\begin{array}{c}\text { Gruppe 3 } \\
\text { Jugendliche } \\
\text { (Moped/Mofa) }\end{array}$ & $\begin{array}{c}\text { Gruppe 4 } \\
\text { Frauen ab 21 }\end{array}$ & $\begin{array}{c}\text { Gruppe 5 } \\
\text { Alle }\end{array}$ \\
\hline Altersgruppen & $19-60$ & alle & $15-20$ & 21 und älter & alle \\
\hline Zeitintervall (Uhrzeit) & $6-8,15-19$ & $6-8,12-21$ & $6-8,10-20$ & $8-21$ & alle \\
\hline Wochentage & Mo - Fr & Mo - Fr & Mo - Fr & Mo - So & alle \\
\hline Monate & April - Oktober & April - Oktober & April - Oktober & April - Oktober & alle \\
\hline Verkehrsbeteiligungsart & $11,12,15$ & $1,2,11,12,15$ & 1,2 & 11,15 & $1,2,11,12,15$ \\
\hline Dauer der Fahrerlaubnis & alle & bis 24 Monate & alle & alle & alle \\
\hline Geschlecht & alle & alle & alle & weiblich & alle \\
\hline
\end{tabular}

Tabelle 4-1: Kriterien der Sonderabfrage beim Statistischen Bundesamt für spezielle Zielgruppen 
ersichtlich. Bei diesen drei Gruppen ist innerorts wie außerorts (ohne BAB) prozentual ein ähnlich hoher Anstieg zu verzeichnen. Die Unfälle bei den Jugendlichen sind leicht um 2 \% zurückgegangen. Auch hier ist der Rückgang innerorts wie auf Landstraßen festzustellen. Bei allen $U(P)$ mit motorisierter Zweiradbeteiligung gab es von 2004 zu 2005 einen Anstieg von 1 \%. Dieser resultiert vor allem aus dem Anstieg im Innerortsbereich. Die prozentual hohen Veränderungen auf Autobahnen sind in den geringen Fallzahlen begründet.

Die Veränderungen in der Entwicklung von 2004 zu 2005 in Bezug auf die Unfälle bei schlechten Lichtverhältnissen (Dämmerung/Dunkelheit) sowie ungünstigem Straßenzustand (nass, winterglatt) können den Diagrammen in Anlage 01 entnommen werden. Die wichtigsten Punkte lassen sich folgendermaßen zusammenfassen:
- Rückgang der Unfälle bei ungünstigem Straßenzustand in allen Zielgruppen sowie für alle $U(P)$ mit motorisierter Zweiradbeteiligung (5\%). Dabei fällt der Rückgang in allen Zielgruppen deutlicher als der Gesamttrend aus (Jugendliche $15 \%$, Fahranfänger $12 \%$, Frauen $9 \%$, Berufsfahrer $8 \%$ ). Ein Grund dafür ist, dass nur die Unfälle April bis Oktober in den Zielgruppen erfasst werden und somit die Schlechtwetterunfälle im Winter darin nicht enthalten sind.

- Der relative Rückgang bezogen auf alle U (P) mit motorisierter Zweiradbeteiligung fällt auf Landstraßen doppelt so hoch aus wie innerorts.

- Die Unfälle bei schlechten Lichtverhältnissen bleiben insgesamt nahezu unverändert (Anstieg um 1 \%). Über alle Ortslagen ergibt sich für die Berufsfahrer und Fahr-

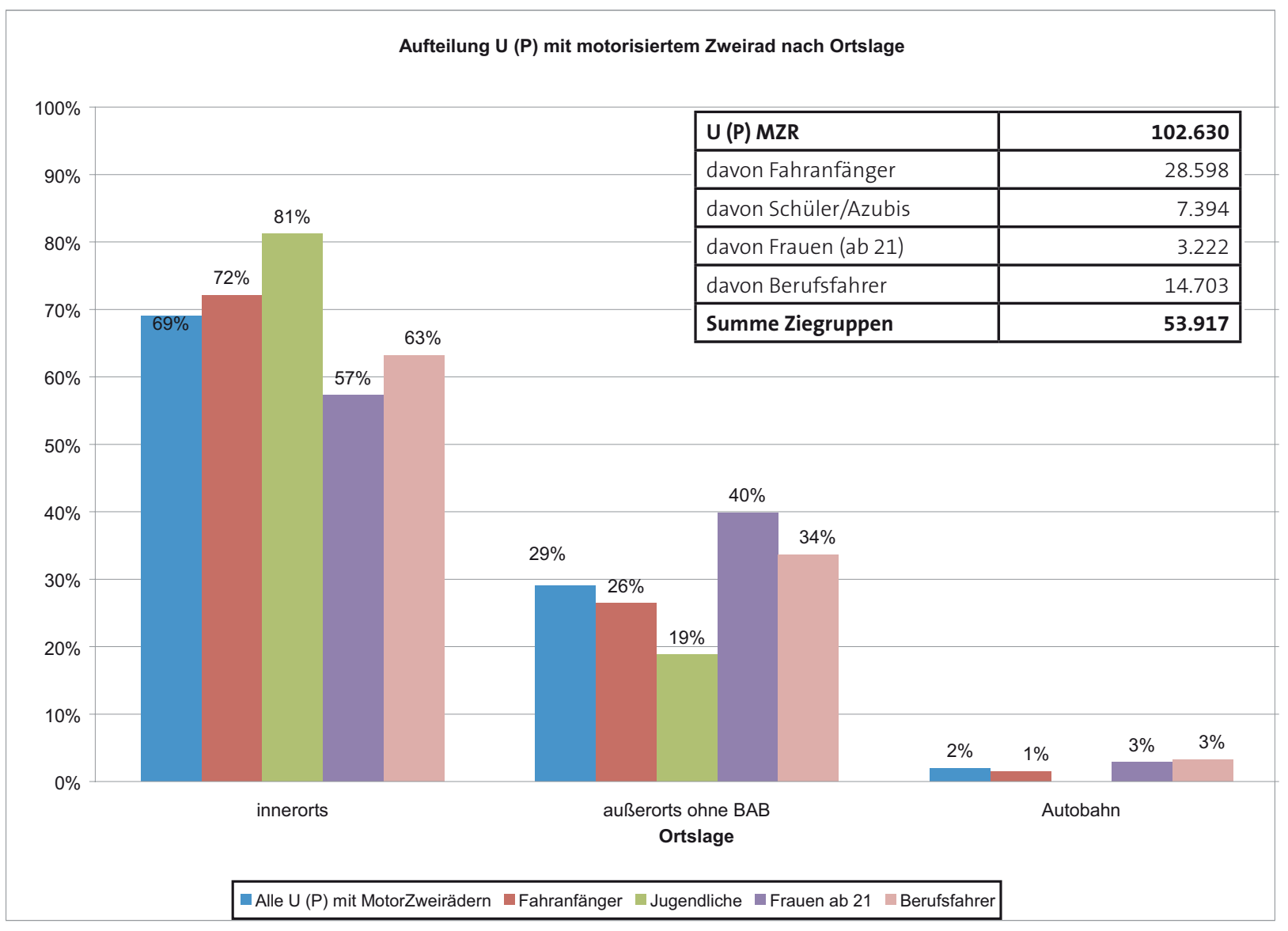

Abbildung 4-10: Aufteilung der U (P) der Zielgruppen nach Ortslage - Bundesstatistik 2004 und 2005 
anfänger ein Anstieg um 23 \% bzw. 10 \%, während die Unfallzahlen für die Jugendlichen und Frauen zurückgehen (um $3 \%$ bzw. $14 \%$ ).

- Die relativen Veränderungen sind bei den Zielgruppen auf Landstraßen deutlich größer, die Fallzahlen sind dort aber gering. Ausnahme bilden die Berufsfahrer, bei denen sowohl innerorts als auch außerorts (ohne BAB) ein Anstieg über $20 \%$ zu verzeichnen ist.

Relative Veränderung der Unfallhäufigkeit von 2004 zu 2005 U (P) mit motorisiertem Zweirad nach Ortslage

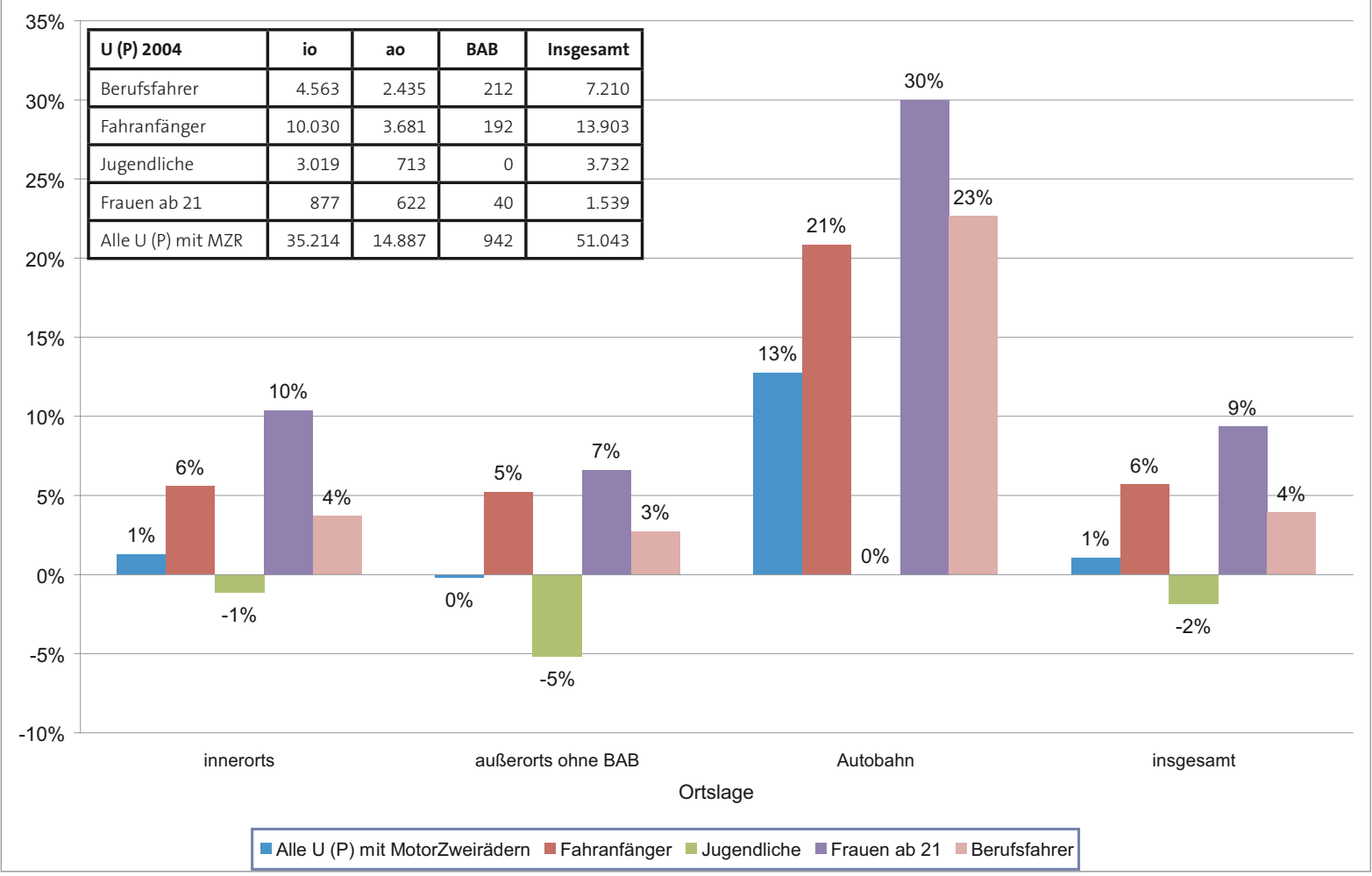

Abbildung 4-11: Relative Veränderung der Unfallhäufigkeit von 2004 zu 2005 für die Zielgruppen differenziert nach Ortslage 


\section{Unfallgeschehen und Straßeneigenschaften}

\subsection{Methodik}

\subsubsection{Untersuchungsgebiet}

Für die Untersuchung des Einflusses von Straßeneigenschaften auf das Unfallgeschehen von motorisierten Zweirädern wurde das Bundesland Sachsen ausgewählt. Hierfür sind Unfalldaten einschließlich der Beschreibung des Unfallherganges nach Verkehrsunfallanzeige (VUA) für die Jahre 2004-2006 verfügbar. Für Unfalldaten der Jahre 2003 und 2004 wurde anhand der einwohnerbezogenen Kenngrößen Getötetenbelastung, Verunglücktenbelastung, und Fahrzeugbestandsbelastung überprüft, ob Unfallgeschehen und Fahrzeugbestand von motorisierten Zweirädern in Sachsen im Vergleich zum Bundesdurchschnitt deutliche Abweichungen aufweisen. Dabei wurden nur Unfälle mit Beteiligung von Motorrädern betrachtet.
Aus der Abbildung ist erkennbar, dass Sachsen bei der Getötetenbelastung das Bundesland ist, welches in beiden Jahren dem Bundesdurchschnitt am nächsten kommt. Bei Betrachtung der Verunglücktenbelastung (Abbildung 5-2) sind die Abweichungen etwas größer, Sachsen stellt jedoch keinen Ausreißer dar.

Der Blick auf den Fahrzeugbestand in Abbildung 5-3 zeigt eine sehr deutliche Abweichung vom Bundesdurchschnitt.

Es sind allerdings zwei deutlich unterschiedliche Gruppen von jeweils acht Bundesländern zu erkennen, die in sich weitgehend vergleichbare Bestandszahlen aufweisen. Sachsen ist repräsentativ für die Gruppe der neuen Bundesländer und Stadtstaaten.

Zusätzlich wurde die Verteilung der Unfallschwere aller Unfälle mit PTW-Beteiligung der Unfallkategorien 1 bis 4

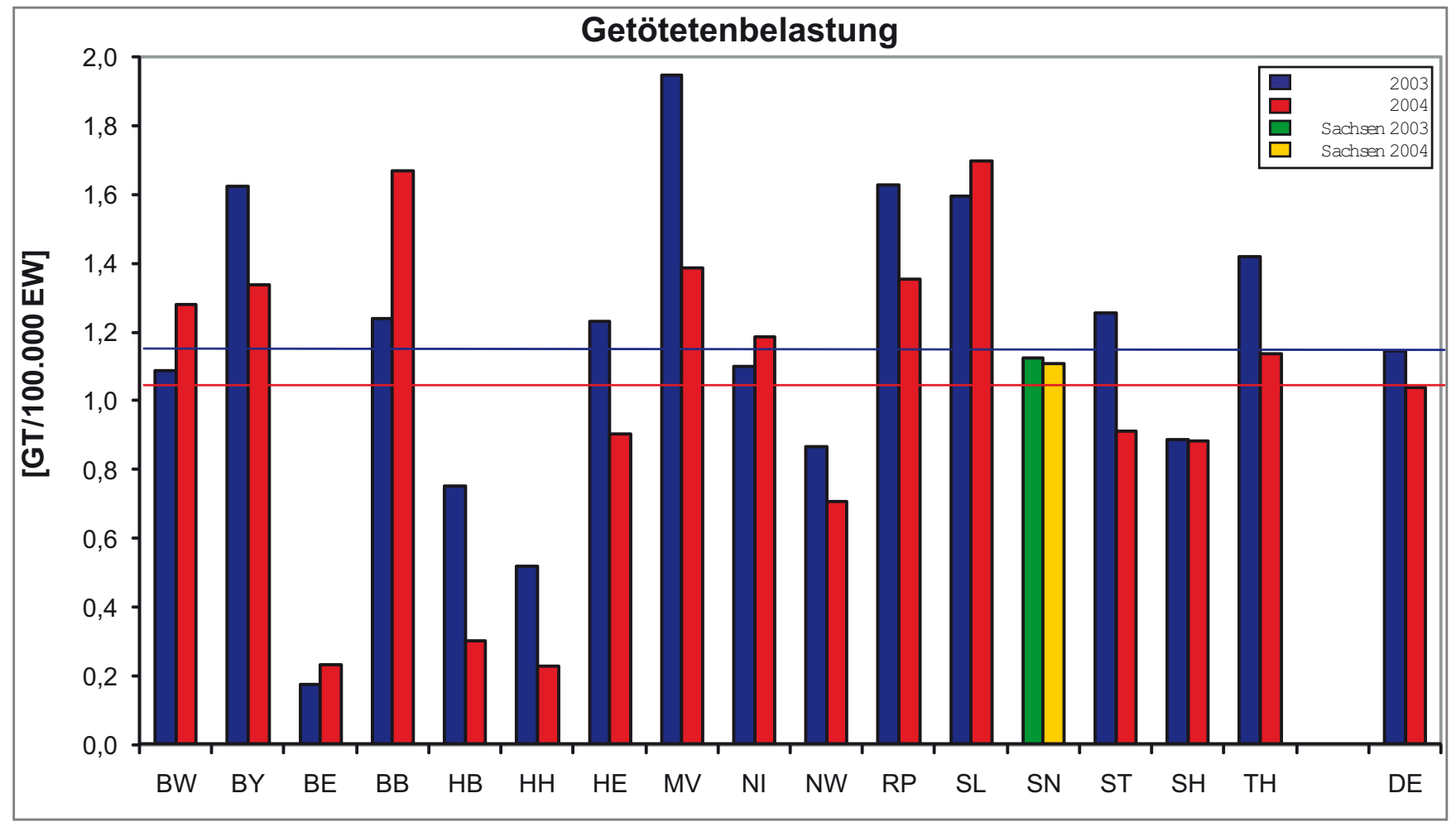

Abbildung 5-1: Getötetenbelastung nach Bundesländern für die Unfälle mit Motorradbeteiligung 2003 und 2004 


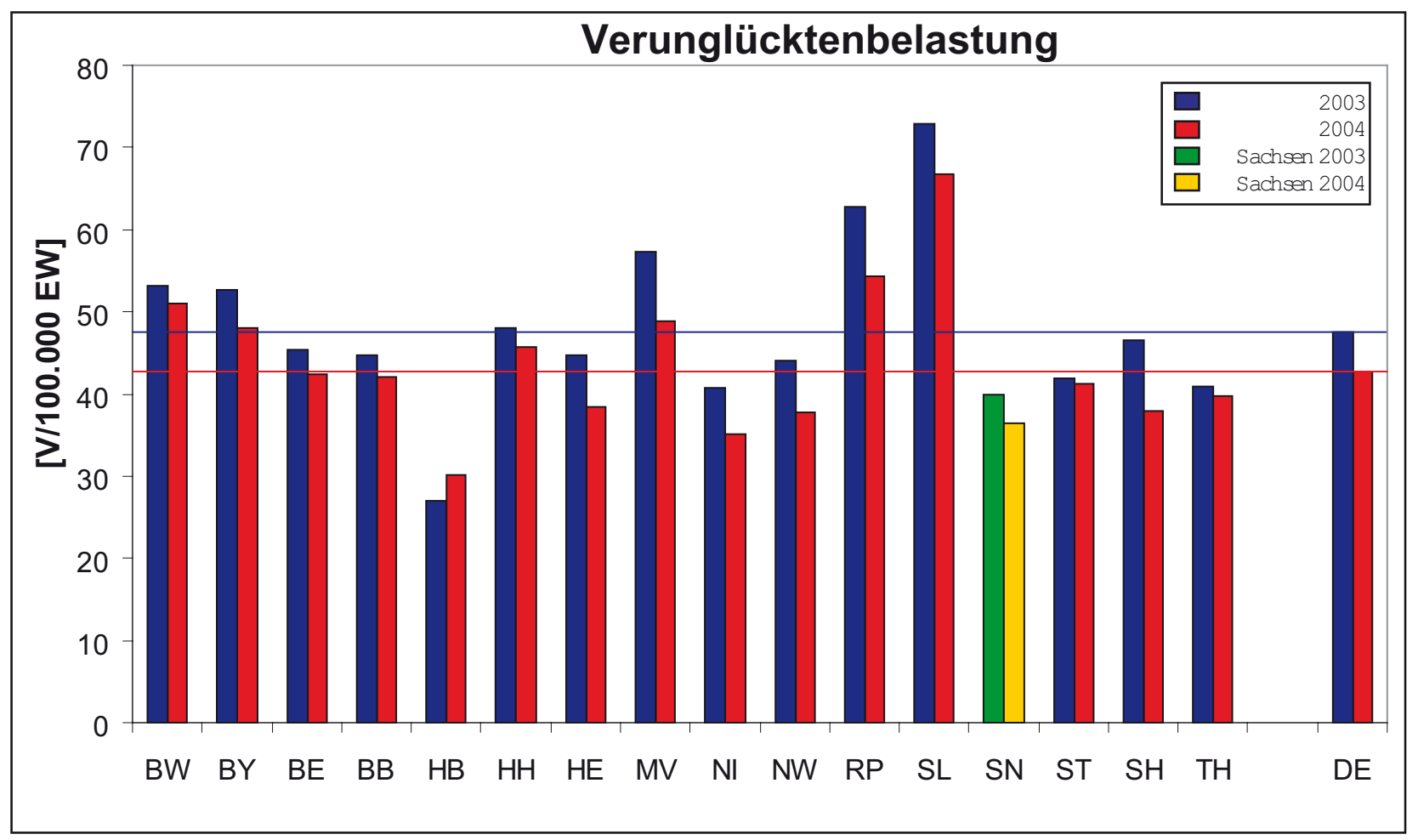

Abbildung 5-2: Verunglücktenbelastung nach Bundesländern für die Unfälle mit Motorradbeteiligung 2003 und 2004

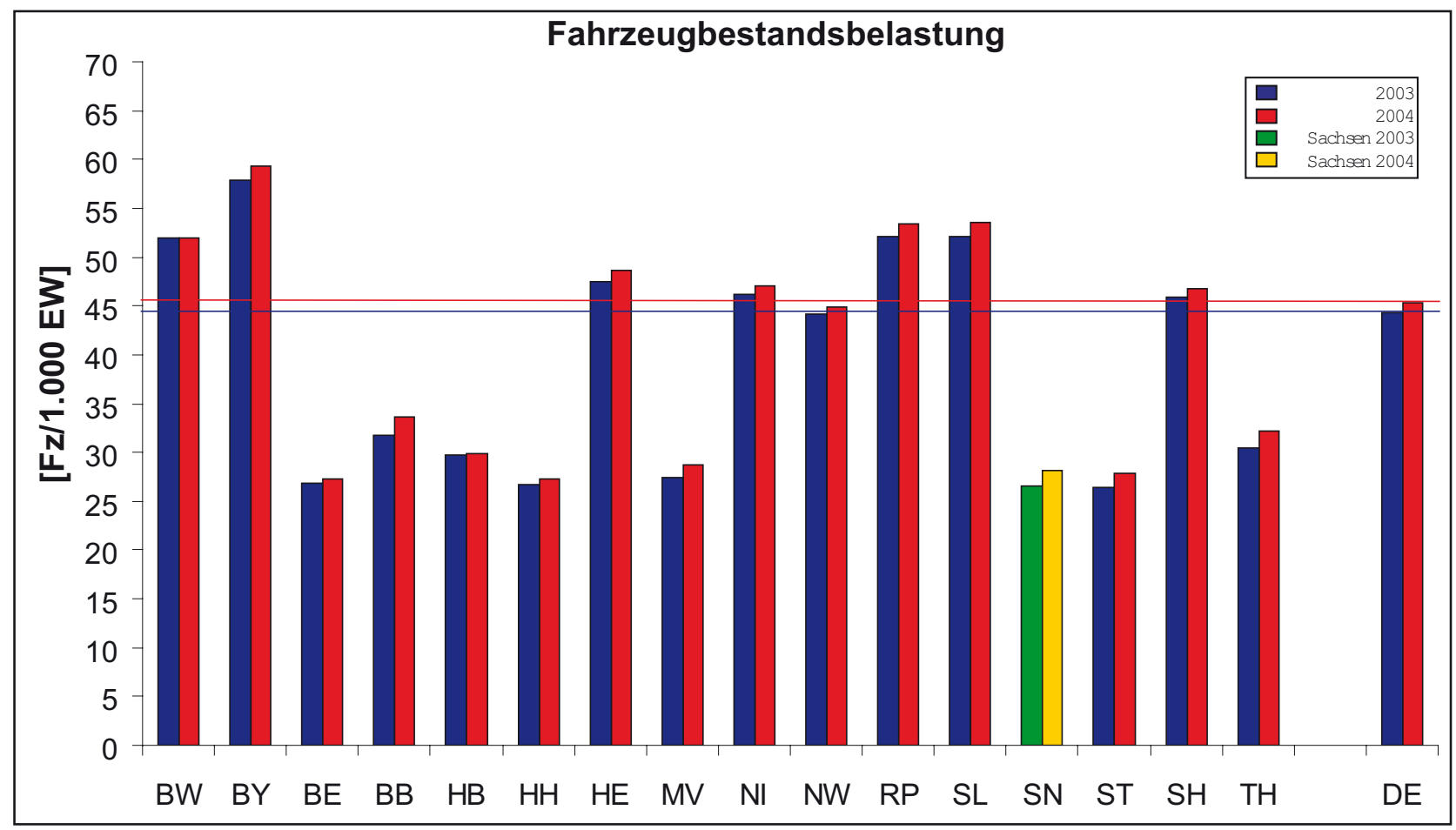

Abbildung 5-3: Fahrzeugbestandsbelastung nach Bundesländern für Motorräder 2003 und 2004 
von Sachsen und dem gesamten Bundesgebiet für das Jahr 2005 differenziert nach Ortslage verglichen (Abbildung 5-4).

Der Anteil der Unfälle mit schwerwiegendem Sachschaden im engeren Sinne befindet sich für Sachsen und Deutschland in einer ähnlichen Größenordnung. Dies gilt für alle Ortslagen. Innerorts fallen in Sachsen die Unfallfolgen schwerer als im Bundesdurchschnitt aus, der Anteil der Unfälle mit schwerem Personenschaden ist um ca. fünf Prozentpunkte größer. Auf Landstraßen ergibt sich ein ähnliches Bild. Die deutlichen Unterschiede in der Unfallschwere auf Autobahnen sind wegen der für diese Ortslage geringen absoluten Unfallanzahl in Sachsen nicht bedeutsam.

Die Betrachtungen zeigen insgesamt, dass Sachsen keine auffällig hohen Abweichungen vom Bundesdurchschnitt verzeichnet und als repräsentatives Untersuchungsgebiet geeignet ist. Somit ist prinzipiell die Übertragbarkeit der in Sachsen erzielten Ergebnisse auf andere Gebiete in Deutschland zulässig. Regionale Besonderheiten sind dabei aber immer zu berücksichtigen. Eine ergänzende Untersuchung könnte also sinnvoll sein, um die Unterschiede in der Fahrzeugbestandsbelastung zu anderen Bundesländern abzuklären.
Da das Unfallkollektiv für die geplanten Untersuchungen schrittweise weiter unterteilt und somit verkleinert wird, sind die maßgebliche Kennwerte der Teilkollektive wie Unfallschwere, Aufteilung auf Unfalltypen und Charakteristik der Unfallstelle mit den Werten der übergeordneten Kollektive vor einer Übertragung der Resultate zu vergleichen.

\subsubsection{Auswahl und Auswertung der Strecken- abschnitte in Sachsen}

Ziel bei der Auswahl von Streckenabschnitten für eine ortsbezogene Detailanalyse von Unfällen mit Beteiligung von PTW ist es, sowohl Abschnitte mit einer geringen Unfalldichte als auch Strecken mit einer hohen Unfalldichte zu erhalten, um festzustellen, in welchen Straßeneigenschaften sich diese Abschnitte unterscheiden. Grundlage bilden das Bundes- und Staatsstraßennetz im Freistaat Sachsen sowie die dazugehörigen polizeilich erfassten Unfälle mit PTW-Beteiligung im Dreijahreszeitraum 2004 bis 2006 wie sie in EUSka lokalisiert sind. Die Auswahl der Abschnitte erfolgte anhand der Kriterien „Ortslage“ und „optische Unfalldichte“. Ist auf der Steckkarte eine Motorradstrecke mit einer Häufung von Unfällen mit Beteiligung von PTW ersichtlich, wird ein Abschnitt definiert. Das Ende eines Abschnitts ergibt sich bei einem Wechsel
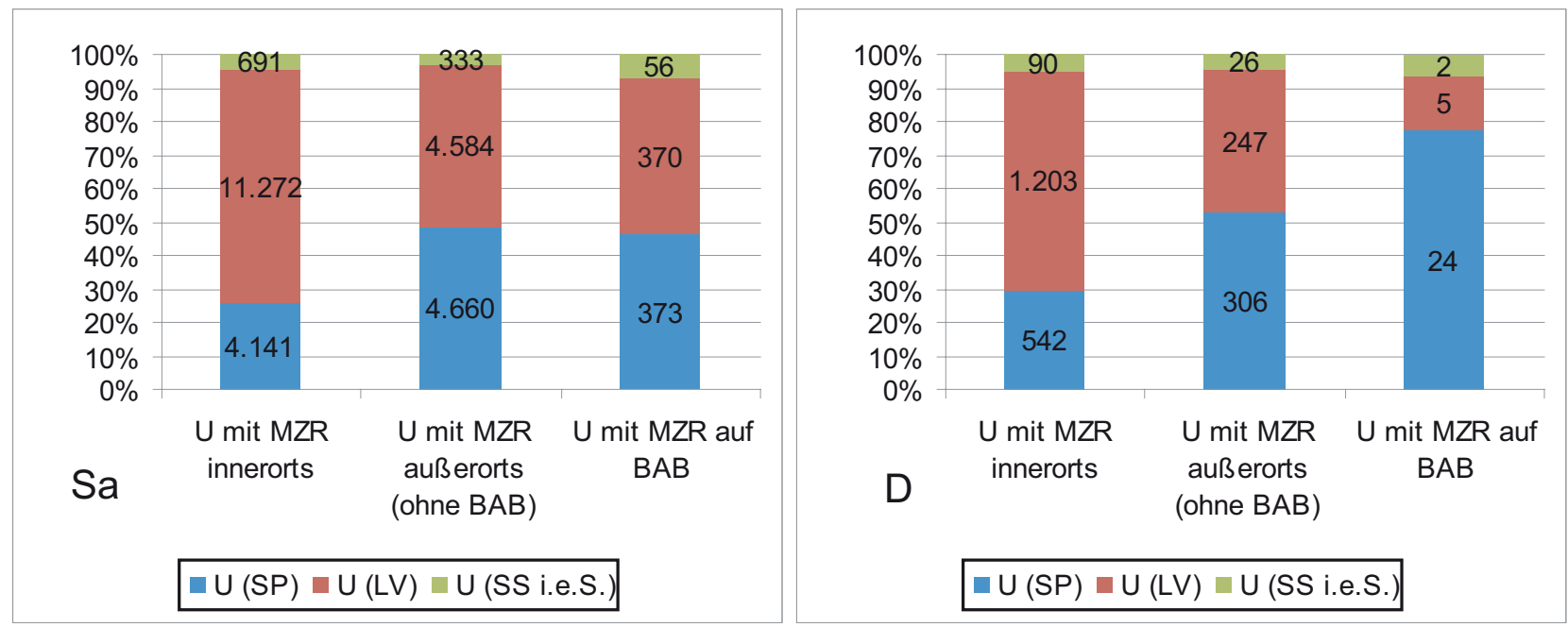

Abbildung 5-4: Vergleich der Unfallschwereverteilung in Sachsen und Deutschland für das Jahr 2005 - U (P+SS i.e.S.) mit Beteiligung motorisierter Zweiräder 
der Ortslage oder bei einer Änderung der optischen Unfalldichte. Zum Vergleich wurden angrenzende Abschnitte mit geringer Unfalldichte entsprechend festgelegt.

Das Resultat sind 219 Abschnitte (Lage in Sachsen siehe Anlage 02), für die das Unfallgeschehen differenziert nach Ortslage und Unfalldichte der Strecken ausgewertet wurde (Tabelle 5-1).

Maßgebend für die Zuordnung zu Strecken mit hoher bzw. geringer Unfalldichte ist die Unter- oder Überschreitung der mittleren Unfalldichte der ausgewählten Innerorts- bzw. Außerortsabschnitte: UD = 1,4 (io) und 0,5 (ao) in Unfällen pro Kilometer und Jahr. Daraus ergeben sich folgende Teilkollektive, bei denen die Strecken mit hoher Unfalldichte generell mehr als doppelt so viele Unfälle je Kilometer aufweisen als Strecken mit geringer Unfalldichte (Tabelle 5-2).

Für diese Streckenabschnitte und ihre Unfallkollektive wurden bestimmte Merkmale untersucht:

- Anteile der Unfälle bestimmter Unfallkategorien:

$$
\cup(G T), \cup(S P), U(P)
$$

- Anteile der Unfälle bestimmter Unfalltypen: Fahrunfall (F), Abbiegeunfall (AB), Einbiegen/Kreuzen-Unfall (EK), Unfall im Längsverkehr (LV)

- Anteile der Unfälle bei Schlechtwetterbedingungen (Straßenzustand nass/feucht oder winterglatt bzw. Unfälle in den Monaten November bis März)

- Anteil der Unfälle bei schlechten Lichtverhältnissen (Dämmerung oder Dunkelheit)

- Anteile der Unfälle am Wochenende (Wochentage Samstag und Sonntag)

- Anteile der Unfälle an charakteristischen Örtlichkeiten: Knoten (Kreuzung + Einmündung), Kurve, Steigung, Gefälle

- Anteil der Alleinunfälle und Anteil der Unfälle mit Fahrer des PTW als Hauptverursacher nach VUA (Beteiligter 01)

- Anteile der definierten Zielgruppen (vgl. Abschnitt 1.3) an den am Unfall beteiligten Fahrer des PTW.

Für jedes Auswertungskriterium wie z. B. der Anteil der Unfälle in Kurven (nach Charakteristik der Unfallstelle in der VUA) wurde eine Vierfeldertabelle folgender Art erstellt (Tab. 5-3).

\begin{tabular}{|c|c|c|c|}
\hline \multicolumn{4}{|c|}{ Anzahl Abschnitte } \\
\hline & geringe UD & hohe UD & Summe \\
\hline Innerorts & 70 & 46 & 116 \\
\hline Außerorts & 52 & 51 & 103 \\
\hline Summe & 122 & 97 & 219 \\
\hline \multicolumn{4}{|c|}{ mittlere Abschnittslänge in [km] } \\
\hline & geringe UD & hohe UD & Summe \\
\hline Innerorts & 2,6 & 2,2 & 2,4 \\
\hline Außerorts & 3,5 & 2,2 & 2,9 \\
\hline alle & 3,0 & 2,2 & 2,6 \\
\hline
\end{tabular}

Tabelle 5-1: Abschnitte in Sachsen differenziert nach Ortslage und Unfalldichte

\begin{tabular}{|c|c|c|c|}
\hline \multicolumn{4}{|c|}{ Unfallanzahl 2004 - 2006 } \\
\hline & geringe UD & hohe UD & Summe \\
\hline Innerorts & 473 & 726 & 1.199 \\
\hline Außerorts & 151 & 272 & 423 \\
\hline Summe & 624 & 998 & 1.622 \\
\hline \multicolumn{4}{|c|}{ Unfalldichte in [U/(km x a)] } \\
\hline & geringe UD & hohe UD & Summe \\
\hline Innerorts & 2,6 & 2,2 & 2,4 \\
\hline Außerorts & 3,5 & 2,2 & 2,9 \\
\hline alle & 3,0 & 2,2 & 2,6 \\
\hline
\end{tabular}

Tabelle 5-2: Unfallanzahl und Unfalldichte der festgelegten Abschnitte

\begin{tabular}{|c|c|c|c|}
\hline \multicolumn{4}{|c|}{ Anteil Fahrunfall } \\
\hline & geringe UD & hohe UD & Summe \\
\hline Innerorts & $19 \%$ & $14 \%$ & $16 \%$ \\
\hline Außerorts & $48 \%$ & $47 \%$ & $47 \%$ \\
\hline alle & $28 \%$ & $25 \%$ & $26 \%$ \\
\hline
\end{tabular}

Tabelle 5-3: Beispiel einer Vierfeldertabelle 
Die Darstellungsform ermöglicht den gleichzeitigen Vergleich der jeweiligen Anteile der Streckenabschnitte differenziert nach Ortslage und Unfalldichte. Das Beispiel zeigt einen erheblich höheren Anteil der Fahrunfälle auf Landstraßen als auf Stadtstraßen, aber unabhängig von der Höhe der Unfalldichte in beiden Ortslagen.

Es erfolgte eine Auswertung der Merkmale sowohl für alle Unfälle als auch ausschließlich für die Unfälle mit Personenschaden mit PTW-Beteiligung in der 3-Jahreskarte 2004-2006. Um Unterschiede in Bezug auf das gesamte Unfallgeschehen im Straßenverkehr zu ermitteln, wurden zusätzlich die Unfälle mit Personenschaden aller Verkehrsteilnehmer auf den untersuchten Streckenabschnitten nach den oben aufgeführten Merkmalen ausgewertet.

\subsubsection{Streckenbesichtigungen}

\subsubsection{Auswahl der Streckenabschnitte}

Aus den 219 Abschnitten wurden 126 Streckenabschnitte zur Ortsbesichtigung sowie zur Aufnahme von Straßeneigenschaften ausgewählt, die eine besonders hohe bzw. geringe Unfalldichte aufweisen, damit später die Unterschiede im Unfallgeschehen möglichst klar hervortreten. Maßgebliches Kriterium war hierbei die mittlere Unfalldichte der 219 Abschnitte aus der 2-Jahreskarte der Unfälle mit Personenschaden 2004 bis 2005, da für 2006 zum damaligen Zeitpunkt noch nicht die Unfalldaten ausgewertet waren. Für die Außerortsstrecken beträgt der Wert 0,4 Unfälle pro Kilometer und Jahr, für die Innerortsstrecken 0,9 Unfälle pro Kilometer und Jahr. Nach dieser Auswahl wurde eine effiziente Route zur Besichtigung festgelegt.

Besichtigte Streckenabschnitte wurden in feinere Bereiche untergliedert, wenn bezüglich einer wesentlichen Straßeneigenschaft wie z. B. Fahrbahnbreite oder Querschnittsaufteilung eine erhebliche Änderung festgestellt wurde. Dies führte zu kürzeren Längen als bei allen Abschnitten in Tabelle 5 1. Zudem erfolgte - wenn nötig eine Korrektur der Zuordnung nach Ortslage anhand der Position der Ortstafeln. Anschließend wurden für die aus der Besichtigung resultierenden Innerorts- und AuBerortsabschnitte eine mittlere Unfalldichte auf Basis der Unfälle mit Personenschaden und unter Einbezug der Unfälle aus 2006 berechnet und anhand dieser eine neue Klassierung vorgenommen. Die Abschnitts- und Unfallzahlen, die der Untersuchung des Einflusses von Straßeneigenschaften auf das Unfallgeschehen zugrunde liegen, ergeben sich dann wie folgt:

\begin{tabular}{|c|c|c|c|}
\hline \multicolumn{4}{|c|}{ Anzahl Abschnitte } \\
\hline & geringe UD & hohe UD & Summe \\
\hline Innerorts & 46 & 40 & 86 \\
\hline Außerorts & 20 & 20 & 40 \\
\hline Summe & 66 & 60 & 126 \\
\hline \multicolumn{4}{|c|}{ mittlere Abschnittslänge in [km] } \\
\hline & geringe UD & hohe UD & Summe \\
\hline Innerorts & 0,8 & 1,5 & 1,1 \\
\hline Außerorts & 2,0 & 1,9 & 1,9 \\
\hline alle & 1,2 & 1,6 & 1,4 \\
\hline \multicolumn{4}{|c|}{ Unfallanzahl 2004 - 2006} \\
\hline & geringe UD & hohe UD & Summe \\
\hline Innerorts & 140 & 281 & 421 \\
\hline Außerorts & 24 & 85 & 109 \\
\hline Summe & 164 & 366 & 530 \\
\hline \multicolumn{4}{|c|}{ Unfälle mit Personenschaden 2004 - 2006} \\
\hline & geringe UD & hohe UD & Summe \\
\hline Innerorts & 74 & 181 & 255 \\
\hline Außerorts & 16 & 73 & 89 \\
\hline Summe & 90 & 254 & 344 \\
\hline \multicolumn{4}{|c|}{ Unfalldichte in [U (P)] / [km x a] } \\
\hline & geringe UD & hohe UD & Summe \\
\hline Innerorts & 0,7 & 1,0 & 0,9 \\
\hline Außerorts & 0,1 & 0,7 & 0,4 \\
\hline alle & 0,4 & 0,9 & 0,7 \\
\hline
\end{tabular}

Tabelle 5-4: Kenndaten der für die Ortsbesichtigung ausgewählten Streckenabschnitte 


\subsubsection{Aufgenommene Daten}

Die Streckenbesichtigungen fanden im Zeitraum von Juni bis August 2007 statt. Aufgenommen wurden folgende Daten:

- Knotenpunkte

- Knotenlage, Grundform, Betriebsform

- Querschnitt

- Fahrbahnbreite, Mittelstreifen, Nebenflächen

(z. B. Radfahrstreifen, Parkbuchten, Fußwege etc.),

Gleisanlagen, angrenzende Bebauung)

- sonstige bauliche Daten

- Hochpunkte, kreuzende Gleisanlagen

- Straßenzustand

- Fahrbahnmaterial, Auftreten sowie Ausprägung von Spurrinnen, Netzrissen, Ausbrüchen, Flickstellen, sonstigen Oberflächenschäden, Anzeichen für häufige Verschmutzungen (z. B. durch Ausfahrten von Feldern, Dauerbaustellen etc.)

- Straßenausstattung

- relevante Beschilderung, Fahrbahnmarkierung, senkrechte Leiteinrichtungen

- sonstige Merkmale (nur außerorts)

- Hindernisse im Seitenraum, Sichteinschränkungen

Ein Beispiel für ein Aufnahmeprotokoll einschließlich Erläuterungen zu den einzelnen Merkmalsausprägungen ist in Anlage 02 zu finden. Ergänzt wurden die aufgenommen Daten außerorts durch Angaben zu den Kurvenradien aus der Straßendatenbank von Sachsen. Damit konnte die Kurvigkeit der Abschnitte berechnet werden. Für 32 der 40 besichtigten Außerortsabschnitte standen diese Daten zur Verfügung.

\subsubsection{Datenauswertung der besichtigten Strecken- abschnitte}

Bei der Untersuchung der besichtigten Streckenabschnitte ist generell zu berücksichtigen, dass der Zeitpunkt der Aufnahmen (2007) zu dem untersuchten Unfallzeitraum zeitlich versetzt ist (2004-2006), so dass bauliche oder betriebliche Veränderungen in der Zwischenzeit nicht vollständig auszuschließen sind.
Grundsätzlich sind zwei Möglichkeiten denkbar, um anhand des zur Verfügung stehenden Datenmateriales einen Zusammenhang zwischen Unfallgeschehen und Straßeneigenschaften zu ermitteln:

- Klassifizierung der Abschnitte nach ähnlichen Straßeneigenschaften mit anschließendem Vergleich des zugehörigen Unfallgeschehens

- Klassifizierung der Abschnitte nach dem Unfallgeschehen mit anschließendem Vergleich der zugehörigen Straßeneigenschaften.

Die erste Variante beinhaltet das Problem, dass die Klassenbildung erfolgen kann entweder

- nach einer Straßeneigenschaft allein (z. B. der Querschnittsbreite) und somit bei dem folgenden Vergleich des Unfallgeschehens der Einfluss der restlichen Eigenschaften nicht berücksichtigt ist,

- oder aber nach Kombinationen wichtiger Eigenschaften (z. B. Querschnittsbreite, Kurvigkeit, Mängel im Straßenzustand), was aber die Anzahl der auszuwertenden Abschnitte pro Klasse sehr stark reduziert.

Deshalb wird die zweite Variante gewählt. Hierbei kann nach einer einfachen Klassenbildung nach dem Unfallgeschehen - in Abschnitte mit hoher und geringer Unfalldichte - anschließend für jede der zwei Klassen die Charakteristik der Straßeneigenschaften herausgearbeitet werden. Anhand der Resultate lassen sich mögliche Einflüsse bestimmter Straßeneigenschaften auf das Unfallgeschehen ableiten. Diese Erkenntnisse bilden die Basis, um für typische Unfallkonstellationen anhand von Detailanalysen der darin enthaltenen Unfälle die Einflüsse auf ihre zu Stichhaltigkeit und Ausprägung zu überprüfen.

Die Bildung der für PTW-Unfälle typischen Unfallkonstellationen erfolgt in einem ersten Schritt über die Unterscheidungsmerkmale Ortslage, Örtlichkeit (Charakteristik der Unfallstelle) und Unfalltyp. Mit der Verwendung dieser Merkmale ist auch eine spätere Zusammenführung der Ergebnisse von Seiten der Verkehrstechnik und von Seiten der Fahrzeugtechnik gewährleistet. Die so erhaltenen Unfallkollektive werden nach einer detaillierten 
Auswertung der Unfallmerkmale und der Unfalltexte (Beschreibung des Unfallhergangs in der VUA) anhand zusätzlicher Merkmale, insbesondere der Unfallart, weiter eingegrenzt. Die entstandenen Kollektive stellen die feinste Grundlage für die vorliegende Untersuchung dar und werden im Blick auf das Unfallgeschehen, die Straßeneigenschaften und die definierten Zielgruppen im Detail analysiert. Kenngrößen wie

- Anteil der Unfälle mit schwerem Personenschaden

- Anteil der Unfälle mit Personenschaden
- Anteil der Unfälle am Wochenende

- Anteil der Unfälle an LSA in Betrieb sowie

- Anteil der Unfälle mit einem PTW als Hauptverursacher

sind mitentscheidend, ob anschließend einzelne Konstellationen sinnvoll zusammengefasst werden können, um die Unfallanzahl pro Kollektiv und somit die Aussagefähigkeit zu erhöhen.

Abbildung 5-5 fasst die Vorgehensweise zusammen:

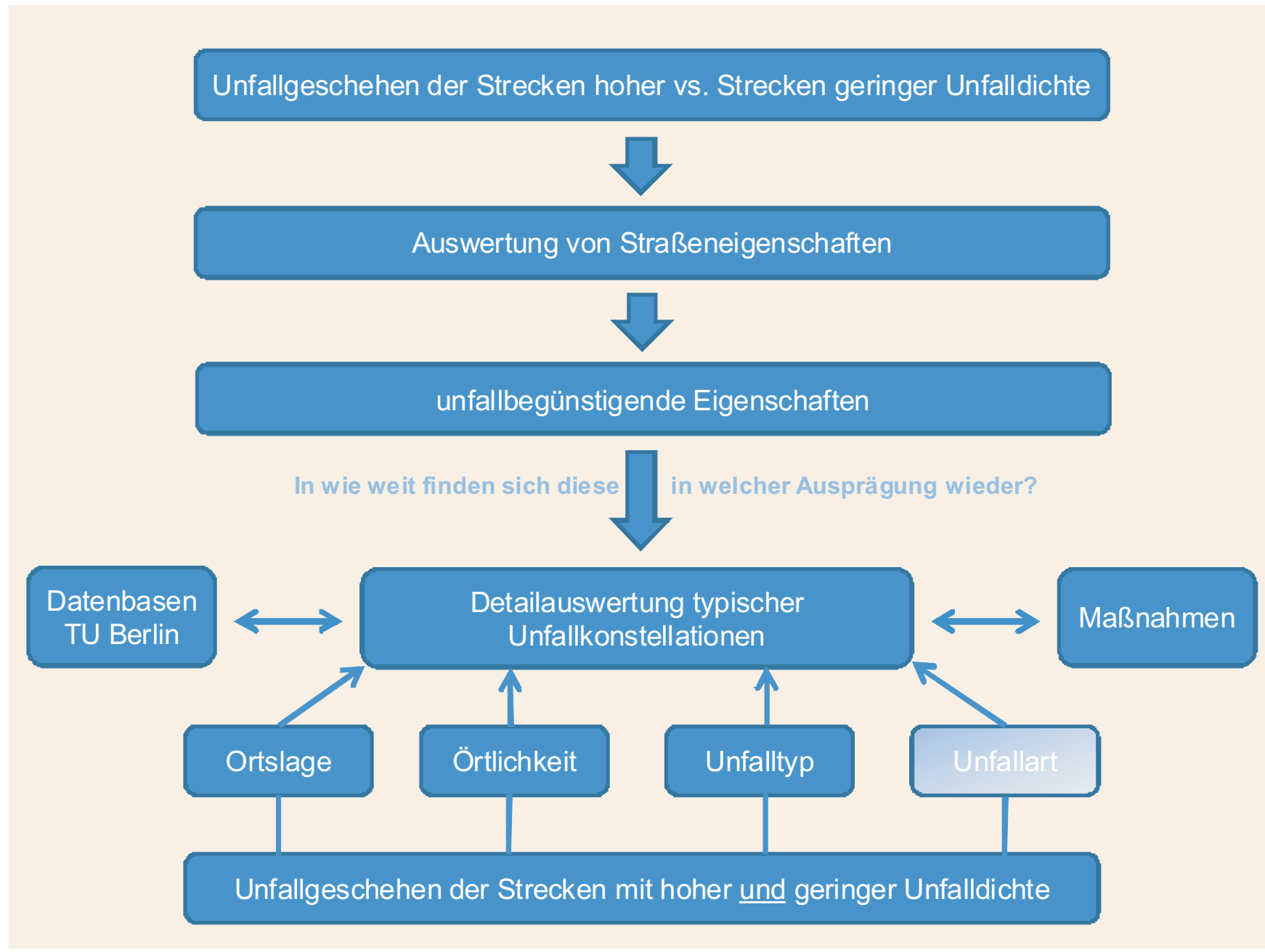

Abbildung 5-5: Vorgehen bei der Auswertung von Daten der besichtigten Motorradstrecken 


\subsection{Ergebnisse}

\subsubsection{Unfallgeschehen in Sachsen}

Das Unfallgeschehen von motorisierten Zweirädern in dem für die Untersuchung relevanten Zeitraum 2004-2006 stellt sich insgesamt mit 11.991 Unfällen, davon 57 \% Unfälle mit Personenschaden (6861), dar. Die Unfälle stellen die Basis für die Festlegung von Streckenabschnitten für eine Detailanalyse von Unfällen mit PTW-Beteiligung dar.

Für das Jahr 2005 wurde für Motorradunfälle in Sachsen die angepasste Unfallschwere in Form von WUa (P) für verschiedene zeitliche und örtliche Charakteristiken verglichen. WUa (P) ist der mittlere Unfallkostensatz für einen Motorradunfall mit Personenschaden, direkt angepasst an die Verunglücktenstruktur 2005 in Sachsen. Das folgende Diagramm zeigt die Kenngröße in Bezug auf die Unfälle mit den Merkmalen nasser oder trockener Straßenzustand, Knotenpunkt (Kreuzung oder Einmündung in der VUA), LSA und Kurve differenziert nach Ortslage (Abbildung 5-6):
Unfälle mit Personenschaden in Kurven sind im Mittel schwerer als alle Motorradunfälle mit verunglückten Personen. Innerorts wie außerorts verzeichnen Unfälle mit Personenschaden bei Nässe im Vergleich zu Unfällen auf trockener Fahrbahn im Mittel eine geringere Schwere. Im Fall einer regennassen Fahrbahn wird möglicherweise bereits vorsichtiger bzw. langsamer gefahren als bei trockenem Straßenzustand, was geringere Kollisionsgeschwindigkeiten zur Folge hat.

Bei allen betrachteten Motorradunfällen mit Personenschaden resultiert im Mittel außerorts eine zweieinhalbfach höhere Unfallschwere als innerorts. Für die Merkmale Knoten und LSA sind die Unterschiede am deutlichsten ausgeprägt. Am geringsten fällt die Differenz für das Merkmal Kurve aus, hier liegt der Innerortswert auch deutlich über dem Mittel aller $U$ (P). Ein Grund wird vor allem in der vergleichsweise zu Knotenüberfahrten im Mittel höheren Geschwindigkeit vermutet, mit welcher der Motorradfahrer die Kurve passiert. Dies wirkt sich bei einem Sturz in der Kurve bzw. einem Abkommen von der Fahrbahn auf die Schwere der Unfallfolgen aus.

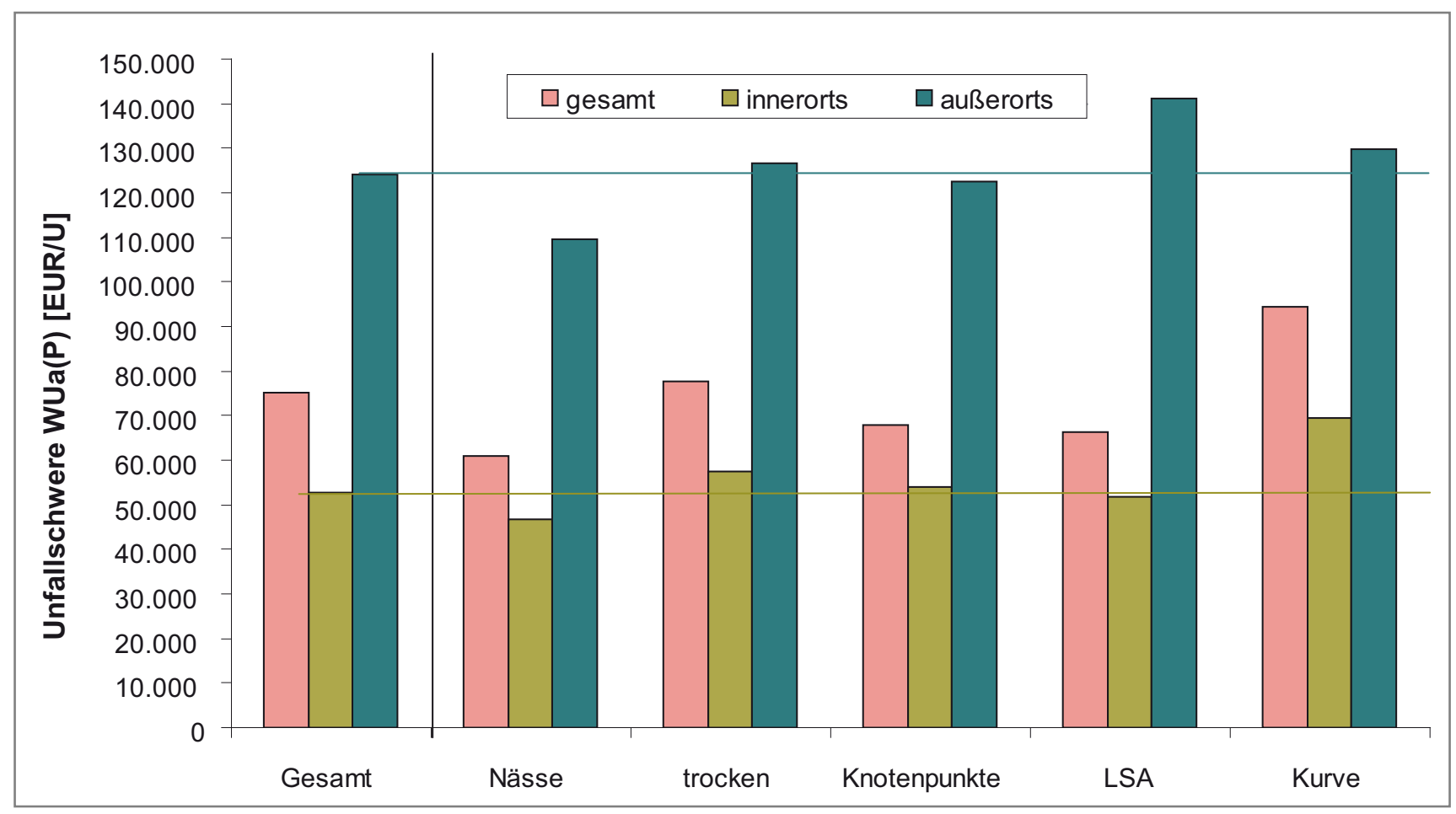

Abbildung 5-6: Unfallschwere der Motorradunfälle nach Straßenzustand, an Knotenpunkten ohne und mit Lichtsignalanlagen sowie in Kurven 
In Abbildung 5-7 werden die Kriterien Tageslicht, Dämmerung, Dunkelheit und Längsneigung untersucht. Im Fall der Unfälle mit Personenschaden an Steigungs- oder Gefällestrecken ist innerorts eine höhere Unfallschwere als im Mittel festzustellen. Außerorts liegt die Unfallschwere der Unfälle bei Dunkelheit und Dämmerung deutlich unterhalb der Unfallschwere außerorts. Auch hier wird vermutet, dass durch die ungünstigen Lichtverhältnisse und somit Sichtbedingungen insgesamt vorsichtiger gefahren wird und die Kollisionsgeschwindigkeiten daher geringer ausfallen. Innerorts dagegen liegen allein die Unfälle bei Dämmerung leicht unter der Unfallschwere innerorts. Die Geschwindigkeitsunterschiede bei unterschiedlichen Lichtverhältnissen sind hier wohl geringfügig und beeinflussen nicht die Unfallschwere.

Die Betrachtung der Unfallschwere bei den Unfalltypen 1-7 zeigt folgendes Bild (Abbildung 5-8):

Der Fahrunfall (Verlust der Fahrzeugkontrolle ohne Einwirkung durch andere Verkehrsteilnehmer) weist im Mittel sowohl außerorts als auch innerorts eine höhere Unfallschwere als alle Unfälle mit Personenschaden auf. Es kann davon ausgegangen werden, dass bei diesem Unfalltyp auch im Mittel die höchsten Geschwindigkeiten vor Eintritt des Unfalles zu beobachten sind. Zusammen mit der Tatsache, dass sich viele Fahrunfälle in der Kurve ereignen und mit einem Aufprall auf ein Hindernis neben der Fahrbahn verbunden sind (vgl. Abschnitt 5.2.4), kann die höhere Unfallschwere erklärt werden. Die Unfälle der anderen Unfalltypen liegen außerorts unter der mittleren Unfallschwere, innerorts weicht der Abbiegeunfall (oft in Verbindung mit Kollision mit dem Gegenverkehr) zusätzlich von der mittleren Unfallschwere aller Unfälle nach oben ab.

Zusammenfassend sind demnach bei Motorradunfällen mit Personenschaden in Kurven, beim Unfalltyp Fahrunfall, an Strecken mit einer Längsneigung und an LSA außerorts besonders schwere Unfallfolgen zu verzeichnen. Inwieweit diese Merkmale kombiniert oder für bestimmte Zielgruppen auftreten können, wird zu einem späteren Zeitpunkt anhand weiter differenzierter Unfallkollektive untersucht.

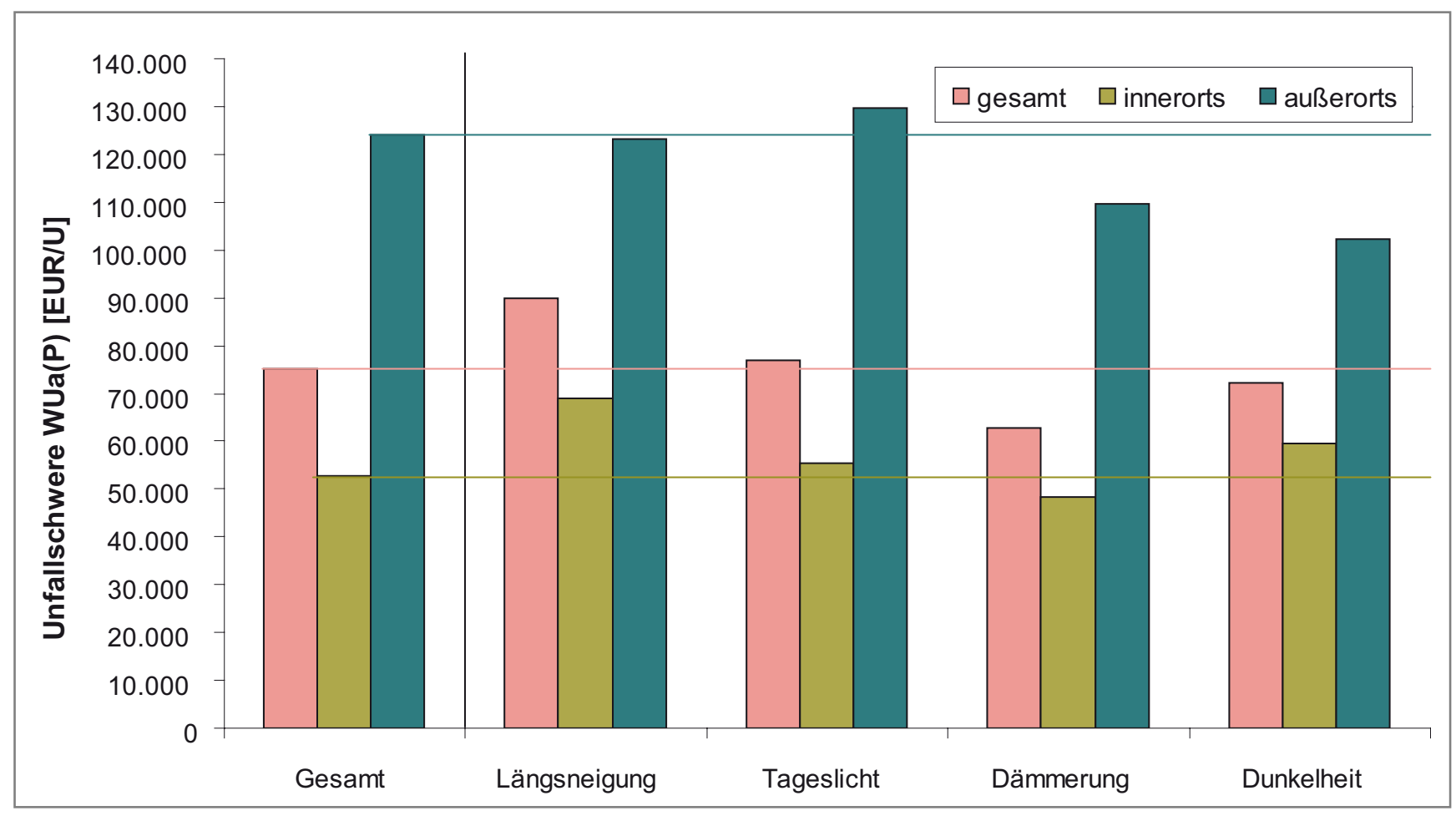

Abbildung 5-7: Unfallschwere der Motorradunfälle auf Straßen mit Längsneigung und nach Lichtverhältnissen 


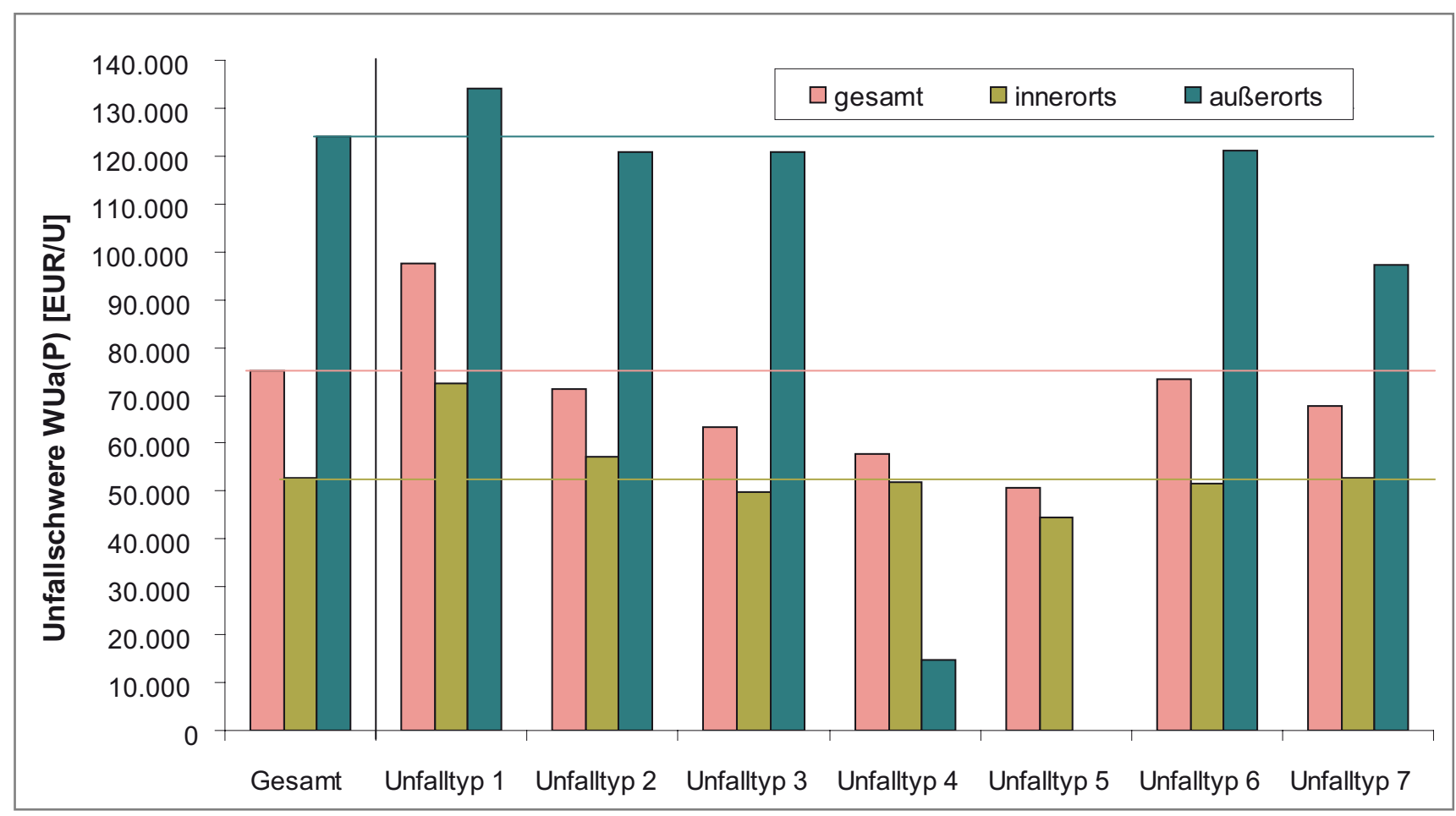

Abbildung 5-8: Unfallschwere der Motorradunfälle nach Unfalltyp

\subsubsection{Unfallgeschehen auf den ausgewählten Streckenabschnitten}

In der 3-JK aller Unfälle mit PTW-Beteiligung 2004-2006 wurden 219 Streckenabschnitte definiert. Deren mittlere Länge, Unfallanzahl und Unfalldichte der Abschnitte wurden in Abschnitt 5.1.2 dargestellt. Die Unfallauswertungen für die Abschnitte differenziert nach Ortslage und Unfalldichte - abgebildet in Vierfeldertabellen (vgl. Abschnitt 5.1.2) - lassen vier verschiedene Vergleichsmöglichkeiten zu, die auch kombiniert werden können:

(1) Unfallgeschehen innerorts vs. Unfallgeschehen außerorts

(2) Unfallgeschehen auf Strecken mit hoher Unfalldichte vs. Unfallgeschehen auf Strecken mit geringer Unfalldichte

(3) Unfallgeschehen aller Unfälle mit PTW vs. Unfallgeschehen der Unfälle mit Personenschaden mit PTW

(4) Unfallgeschehen der U (P) mit PTW vs. Unfallgeschehen $U(P)$ aller Verkehrsteilnehmer.
Im Folgenden werden die wichtigsten Erkenntnisse zusammengefasst dargestellt, die einzelnen Vierfeldertabellen sind in Anlage 04 zusammengestellt.

\section{(1) Unfallgeschehen innerorts vs. Unfallgeschehen außerorts}

Basis: alle Unfälle mit PTW-Beteiligung

- Die Unfalldichte ist innerorts etwa dreifach so hoch wie außerorts.

- Der Anteil der Unfälle mit Getöteten beträgt außerorts das fünfache wie innerorts, der Anteil der Unfälle mit schwerem Personenschaden das zweieinhalbfache und der Anteil der Unfälle mit Personenschaden liegt mit 78 \% um 20 Prozentpunkte höher.

- Der Unfalltyp Fahrunfall ist außerorts mit 42 \% der bestimmende Unfalltyp und weist dort einen dreieinhalbfach höheren Anteil als innerorts auf. Innerorts hingegen sind der Längsverkehrsunfall (LV) mit 35 \% sowie die knotenpunkt- 
stypischen Unfalltypen Abbiegeunfall (AB) und Einbiegen/ Kreuzen-Unfall (EK-Unfall) mit $19 \%$ und $26 \%$ bestimmend. Der EK-Unfall verzeichnet dabei einen fast doppelt so hohen Anteil wie außerorts. Die Unterschiede bei den Typen AB und LV fallen mit außerorts um 4 bzw. 14 Prozentpunkte kleineren Anteilen nicht ganz so deutlich aus.

- Der vor allem am Wochenende und vornehmlich auf Landstraßen stattfindende Ausflugs- und Freizeitverkehr spiegelt sich auch in den Unfallzahlen wieder. Außerorts passieren knapp doppelt so viele Unfälle am Wochenende wie innerorts, insgesamt über $1 / 3$ aller Außerortsunfälle.

- Der Anteil der Unfälle bei Schlechtwetterbedingungen (nasser oder winterglatter Fahrbahnzustand) sowie in den Wintermonaten November bis März ist außerorts mit $19 \%$ bzw. $11 \%$ gegenüber $31 \%$ und $25 \%$ innerorts deutlich geringer und zeigt vor allem, dass bei schlechtem Wetter Ausflugs- und Freizeitverkehr deutlich eingeschränkt stattfindet, während die Gruppe der Berufsfahrer, die vor allem innerorts unterwegs ist, dennoch das PTW als Verkehrsmittel benutzt.

- Die Anteile der Unfälle bei schlechten Lichtverhältnissen unterscheiden sich für die beiden Ortslagen mit 5 Prozentpunkten nicht erheblich.

- Wird der Anteil der Unfälle an charakteristischen Örtlichkeiten verglichen, ist zu beobachten, dass innerorts insbesondere Knotenpunkte mit 52 \% aller Unfälle den Schwerpunkt ausmachen, während außerorts vor allem Kurven mit fast der Hälfte aller Unfälle die Örtlichkeiten darstellen, an denen der Großteil aller Außerortsunfälle passieren. Knotenpunkte und Steigungs-/ Gefällestrecken folgen mit jeweils einem knappen Drittel aller Außerortsunfälle. Mögliche Überschneidungen wurden hierbei nicht berücksichtigt.

- Der Anteil der Unfälle mit einem PTW-Fahrer als Hauptverursacher ist außerorts mit 69 \% 19 Prozentpunkte höher als innerorts. Dies hängt vor allem mit dem höheren Anteil von Alleinunfällen außerorts (etwa vierfach so hoher Anteil wie innerorts) zusammen.
- Bei Betrachtung der Zielgruppen ist folgendes zu erkennen:

- Bei den Jugendlichen ist innerorts ein fast doppelt so großer Anteil Fahrer von PTW an den Unfällen beteiligt wie auf Landstraßen. Die Zielgruppe stellt $1 / 4$ aller beteiligten Fahrer des PTW.

- Der Anteil der anhand der Unfall-Daten eindeutig als Fahranfänger bestimmbaren beteiligten Fahrer von PTW ist mit $20 \%$ zu $14 \%$ ebenso wie der Anteil der Frauen mit $10 \%$ zu $6 \%$ innerorts höher.

- Ähnlich verhält es sich auch bei den Rollerfahrern (3 \% zu 1\%). Dabei sind die Anteile insgesamt aber sehr gering. In der Zielgruppe können aus datentechnischen Gründen nicht alle Rollerfahrer erfasst werden, da nur für Kraftrollerfahrer eine eigene Schlüsselnummer in der Statistik vorgesehen und somit in EUSka auswertbar ist.

- Der Anteil der Zielgruppe ab einem Alter von 55 Jahren ist außerorts mit $8 \%$ geringfügig höher als innerorts mit $6 \%$.

\section{(2) Unfallgeschehen auf Strecken mit hoher Unfall- dichte vs. Unfallgeschehen auf Strecken mit geringer Unfalldichte}

Basis: alle Unfälle mit PTW-Beteiligung innerorts

Ein erkennbarer Unterschied der Anteile hinsichtlich eines höheren Wertes bei den Abschnitten mit hoher Unfalldichte konnte bei den Schlechtwetterunfällen (sowohl Unfälle bei ungünstigem Straßenzustand als auch Unfälle während der Wintermonate), den Unfällen an Knotenpunkten, und den beteiligten Kraftrollerfahrern festgestellt werden.

Basis: alle Unfälle mit PTW-Beteiligung außerorts

Außerorts wurde ein wesentlicher Unterschied der Anteile hinsichtlich eines höheren Wertes bei den Abschnitten mit hoher Unfalldichte bei den Schlechtwetterunfällen (sowohl Unfälle bei ungünstigem Straßenzustand als auch Unfälle während der Wintermonate), den Unfällen an Knotenpunkten und den Unfällen an Strecken mit Längsneigung beobachtet. 
(3) Unfallgeschehen aller Unfälle mit PTW vs. Unfallgeschehen der Unfälle mit Personenschaden mit PTW

Basis Unfälle innerorts:

- Werden nur die Unfälle mit Personenschäden betrachtet, nehmen der Anteil der Fahrunfälle um 4, der Anteil der EK-Unfälle um 6 sowie der Anteil der Abbiegeun-

fälle um 3 Prozentpunkte zu. Der Anteil der Unfälle im Längsverkehr sinkt um $15 \%$.

- Der Anteil der Alleinunfälle steigt um 4 Prozentpunkte.

- Der Anteil der Unfälle am Wochenende bleibt in etwa gleich.

- Erheblich reduziert wird der Anteil der Schlechtwetterunfälle. Der Anteil der Unfälle bei nasser oder winterglatter Fahrbahn verringert sich ebenso wie der Anteil der Unfälle in den Wintermonaten um 12 Prozentpunkte. Dies lässt auf ein vorsichtigeres Verhalten - insbesondere auf geringere Geschwindigkeiten - der Fahrer des PTW bei ungünstigen Witterungsbedingungen schließen, die im Fall eines Unfalles zu weniger schweren Unfallfolgen führen.

- Der Anteil der Unfälle mit einem Fahrer des PTW als Hauptverursacher nach VUA sinkt um 9 Prozentpunkte, d.h. innerhalb von Ortschaften führen die Unfälle, bei denen der Fahrer des PTW Hauptverursacher ist, im Mittel häufiger zu Sachschäden als die Unfälle, bei denen ein anderer Verkehrsteilnehmer Hauptverursacher ist.

- Bei der Analyse konkreter Örtlichkeiten sind für den Anteil der Unfälle (alle vs. U (P)) an Knotenpunkten und an Strecken mit Längsneigung etwa gleichbleibende Anteile zu beobachten. Der Anteil der Unfälle in Kurven steigt um 4 Prozentpunkte. Unfälle in einer Kurve führen demnach öfter zu einem Personenschaden als die Unfälle an anderen Stellen.

- Bei Betrachtung der beteiligten Fahrer des PTW nach Zielgruppen sind keine erheblichen Unterschiede in den Anteilen festzustellen.

Basis Unfälle außerorts:

- Werden die Unfälle mit Personenschäden untersucht, zeigen sich ein zunehmender Anteil von Fahrunfällen
(5 Prozentpunkte), ein gleichbleibender Anteil von EKUnfällen sowie abnehmende Anteile der Abbiegunfälle (2 Prozentpunkte) sowie der Unfälle im Längsverkehr (4 Prozentpunkte).

- Der Anteil der Alleinunfälle steigt um 5 Prozentpunkte.

- Der Anteil der Unfälle am Wochenende bleibt in etwa gleich.

- Wie innerorts verringert sich der Anteil der Schlechtwetterunfälle. Der Anteil der Unfälle bei nasser oder winterglatter Fahrbahn nimmt um 6 und der Anteil der Unfälle in den Wintermonaten um 5 Prozentpunkte $a b$. Daraus kann wie im Innerorts-Fall ein vorsichtigeres Fahrverhalten (geringere Geschwindigkeiten) bei schlechten Witterungsbedingungen vermutet werden. - Der Anteil der Unfälle mit einem Fahrer des PTW als Hauptverursacher nach VUA bleibt gleich.

- Bei der Betrachtung von Örtlichkeiten sind für Unfälle an Knotenpunkten und an Strecken mit Längsneigung in etwa gleichbleibende Anteile festzustellen. Der Anteil der Unfälle in Kurven steigt wie innerorts um 4 Prozentpunkte.

- Der Blick auf die Zielgruppen zeigt keine wesentlichen Abweichungen in den Anteilen.

\section{(4) Unfallgeschehen der U (P) mit PTW vs. Unfallgesche- hen $U(P)$ aller Verkehrsteilnehmer}

Basis Unfälle innerorts:

- Die Unfalldichte der Unfälle mit allen Verkehrsteilnehmern beträgt über das fünffache gegenüber der Dichte der Unfälle mit motorisierten Zweirädern, d.h. das Unfallgeschehen von PTW macht $18 \%$ des gesamten Unfallgeschehens der ausgewählten Innerortsabschnitte aus.

- Wird das Verhältnis der Unfalldichten der Abschnitte mit hoher zu den Abschnitten mit niedriger Unfalldichte untersucht, zeigt sich mit 2,9 für die Unfälle aller Verkehrsteilnehmer gegenüber 2,6 für die Unfälle mit PTW-Beteiligung ein leicht höherer Wert. Die Unfallauffälligkeit bzw. Nichtauffälligkeit der festgelegten Abschnitte ist im gesamten Unfallgeschehen wiederzufinden, sogar noch ein wenig deut- 
licher. Es scheinen demnach unfallbegünstigende Faktoren - möglicherweise bestimmte Straßeneigenschaften - zu existieren, die nicht nur speziell für die Fahrer motorisierter Zweiräder ein erhöhtes Unfallrisiko bergen.

- Der Anteil der Unfälle mit Getöteten bleibt gleich, der Anteil der Unfälle mit schwerem Personenschaden nimmt bei den Unfällen mit PTW-Beteiligung um 8 Prozentpunkte zu.

- Die Unfalltypen Fahrunfall, EK-Unfall und Abbiegeunfall treten bei den Unfällen mit PTW-Beteiligung häufiger auf (Zunahme der Anteile um 5, 4 und 5 Prozentpunkte), der Typ Unfall im Längsverkehr seltener (Abnahme um 8 Prozentpunkte).

- Der Anteil der Unfälle am Wochenende befindet sich in derselben Größenordnung.

- Der Anteil der Alleinunfälle nimmt für die Unfälle mit PTW-Beteiligung um 4 Prozentpunkte zu.

- Der Anteil der Schlechtwetterunfälle nimmt bei den Unfällen mit PTW-Beteiligung deutlich ab (Unfälle bei nasser oder winterglatter Fahrbahn um 10 Prozentpunkte, Unfälle in den Monaten November bis März um 19 Prozentpunkte). Als Grund ist hier vor allem zu sehen, dass die Fahrer des PTW ihr Fahrzeug bei Schlechtwetterverhältnissen nicht benutzen.

- Der Anteil der Unfälle mit PTW-Beteiligung bei ungünstigen Lichtverhältnissen verzeichnet eine leichte Abnahme um 3 Prozentpunkte.

- Der Anteil der Unfälle an Knotenpunkten und an Strecken mit Längsneigung bewegt sich in derselben Größenordnung. Der Anteil der Unfälle in Kurven steigt für die Unfälle mit PTW-Beteiligung um 5 Prozentpunkte. Kurven stellen demnach auch innerorts im Vergleich zu allen Verkehrsteilnehmern ein erhöhtes Unfallpotential für Fahrer des PTW dar.

- Die Betrachtung der Zielgruppen zeigt ein deutliches Bild: Der Anteil der Jugendlichen und Fahranfänger an den beteiligten Fahrern von PTW ist im Vergleich zum gesamten Unfallgeschehen deutlich überrepräsentiert, der Anteil von Frauen und Fahrern ab 55 Jahren deutlich unterrepräsentiert:

\begin{tabular}{|l|l|l|}
\hline Zielgruppe & $\begin{array}{l}\text { Anteil Ziel- } \\
\text { gruppe an } \\
\text { Fahrern von } \\
\text { PTW (nur U (P) } \\
\text { mit PTW-Be- } \\
\text { teiligung) }\end{array}$ & $\begin{array}{l}\text { Anteil Zielgruppe } \\
\text { (Kriterium losgelöst von } \\
\text { der Bedingung ,Fahrer } \\
\text { eines PTW“) an allen } \\
\text { Beteiligten (U (P) aller } \\
\text { Verkehrsteilnehmer) }\end{array}$ \\
\hline Jugendliche & $24 \%$ & $6 \%$ \\
\hline Fahranfänger & $22 \%$ & $9 \%$ \\
\hline Frauen & $12 \%$ & $33 \%$ \\
\hline $\begin{array}{l}\text { Fahrer ab } \\
55 \text { Jahre }\end{array}$ & $7 \%$ & $17 \%$ \\
\hline
\end{tabular}

Tabelle 5-5: Vergleich der Anteile der Zielgruppen - U (P) mit PTW-Beteiligung vs. U (P) aller Verkehrsteilnehmer (innerorts)

Basis Unfälle außerorts:

- Die Unfalldichte bei den Unfällen mit allen Verkehrsteilnehmern beträgt in etwa das dreifache gegenüber der Dichte der Unfälle motorisierter Zweiräder, d. h., das Unfallgeschehen mit PTW macht $28 \%$ des gesamten Unfallgeschehens der ausgewählten Außerortsabschnitte aus.

- Bei Betrachtung des Verhältnisses der Unfalldichten der Abschnitte mit hoher zu den Abschnitten mit geringer Unfalldichte ist der Wert 3 für die Unfälle mit PTW-Beteiligung gegenüber dem Faktor 1,9 für die Unfälle aller Verkehrsteilnehmer festzustellen. Die Unfallauffälligkeit bzw. Nichtauffälligkeit der festgelegten Abschnitte ist folglich auch außerorts im gesamten Unfallgeschehen wiederzufinden, jedoch deutlich abgeschwächt. Es scheinen demnach unfallbegünstigende Faktoren zu existieren, die speziell für die Fahrer motorisierter Zweiräder ein erhöhtes Unfallrisiko bergen.

- Der Anteil der Unfälle mit Getöteten erhöht sich für die Unfälle mit PTW-Beteiligung um 3 Prozentpunkte, der Anteil der Unfälle mit schwerem Personenschaden nimmt um 18 Prozentpunkte zu. Es zeigt sich damit außerorts eine deutliche Zunahme der Schwere der Unfälle mit PTW verglichen mit dem gesamten Unfallgeschehen.

- Der Unfalltyp Fahrunfall tritt bei den Unfällen mit PTW-Beteiligung häufiger auf (Zunahme des Anteils um 6 Prozentpunkte), die Anteile der knotenpunkt- 
spezifischen Unfalltypen EK-Unfall und Abbiegeunfall bleiben in etwa gleich, der Anteil der Unfälle im Längsverkehr nimmt wie innerorts um 8 Prozentpunkte ab.

- Der Anteil der Unfälle am Wochenende erhöht sich auBerorts um 7 Prozentpunkte für die Unfälle mit PTWBeteiligung. Das spiegelt den erhöhten Ausflugs- und Freizeitverkehr von Fahrern der PTW am Wochenende wider, der sich im Unfallgeschehen insbesondere in einer erhöhten Anzahl von Fahrunfällen - meist Alleinunfälle - ausdrückt. Die Zunahme des Anteiles der Alleinunfälle um 8 Prozentpunkte ist daher vor allem so zu erklären.

- Der Anteil der Schlechtwetterunfälle nimmt außerorts in Bezug auf die Unfälle mit PTW-Beteiligung noch stärker ab als innerorts (Unfälle bei nasser oder winterglatter Fahrbahn ebenso wie Unfälle in den Monaten November bis März um 25 Prozentpunkte). Dies zeigt deutlich, dass das motorisierte Zweirad bei Schlechtwetterverhältnissen im Freizeitverkehr kaum benutzt wird, während es innerorts eher bestimmte Nutzergruppen wie die Berufsfahrer gibt, die aufgrund fehlender geeigneter Alternativen auf eine Benutzung des PTW bei schlechtem Wetter nicht verzichten können.

- Der Anteil der Unfälle mit PTW-Beteiligung bei ungünstigen Lichtverhältnissen verzeichnet eine außerorts eine deutliche Abnahme um 12 Prozentpunkte. Die Abweichungen ergeben sich insbesondere daher, dass der Ausflugs- und Freizeitverkehr hauptsächlich zu hellen Tageszeiten bzw. zu den Jahreszeiten, während denen es tagsüber länger hell ist, verkehrt.

- Der Anteil der Unfälle an Knotenpunkten und an Strecken mit Längsneigung befindet sich in einer ähnlichen Größenordnung. Der Anteil der Unfälle in Kurven steigt für die Unfälle mit PTW-Beteiligung um 10 Prozentpunkte. Außerorts beinhalten bekanntermaßen vor allem Kurven ein erhöhtes Unfallpotential für Fahrer von motorisierten Zweirädern.

- Die Betrachtung der Zielgruppen zeigt bezüglich der Relationen ein ähnliches Bild wie innerorts, einzig das Verhältnis bei den Fahranfängern ist weniger deutlich ausgeprägt:

\begin{tabular}{|l|c|l|}
\hline Zielgruppe & $\begin{array}{l}\text { Anteil Zielgruppe } \\
\text { an Fahrer von } \\
\text { PTW (nur U (P) } \\
\text { mit PTW-Be- } \\
\text { teiligung) }\end{array}$ & $\begin{array}{l}\text { Anteil Zielgruppe } \\
\text { (Kriterium losgelöst von } \\
\text { der Bedingung „Fahrer } \\
\text { eines PTW“) an allen } \\
\text { Beteiligten (U (P) aller } \\
\text { Verkehrsteilnehmer) }\end{array}$ \\
\hline Jugendliche & $13 \%$ & $3 \%$ \\
\hline Fahranfänger & $15 \%$ & $12 \%$ \\
\hline Frauen & $7 \%$ & $24 \%$ \\
\hline $\begin{array}{l}\text { Fahrer ab } \\
55 \text { Jahre }\end{array}$ & $10 \%$ & $16 \%$ \\
\hline
\end{tabular}

Tabelle 5-6: Vergleich der Anteile der Zielgruppen - U (P) mit PTW-Beteiligung vs. U (P) aller Verkehrsteilnehmer (außerorts)

In einem weiteren Auswertungsschritt wurde für die festgelegten Zielgruppen die Unfalltypenverteilung bestimmt und mit der Verteilung für alle beteiligte motorisierte Zweiradfahrer innerorts bzw. außerorts verglichen. Grundgesamtheit für die Berechnung der Anteile der Unfalltypen bilden alle unfallbeteiligten motorisierten Zweiradfahrer, die der entsprechenden Zielgruppe zugeordnet werden können. Um zumindest eine wenn auch eingeschränkte Aussage über die Wiedereinsteiger zu erhalten, wurde die Verteilung für alle Fahrer mit einem Alter von 35 bis 54 Jahren (jeweils einschließlich) eines motorisierten Zweirades ermittelt. Die Resultate für die Unfälle mit Innerorts- bzw. Außerortslage sind in Anlage 05 zu sehen.

Innerorts ergeben sich im Vergleich zu allen motorisierten Zweiradfahrern folgende Auffälligkeiten:

- Jugendliche sind anteilsmäßig häufiger in Längsverkehrsunfälle verwickelt (4 Prozentpunkte).

- Fahranfänger sind anteilsmäßig häufiger an Einbiegen/ Kreuzen-Unfällen beteiligt (6 Prozentpunkte).

- Schlechtwetterunfälle weisen einen größeren Anteil an Fahrunfällen auf (5 Prozentpunkte).

- Frauen sind anteilsmäßig häufiger bei Fahrunfällen beteiligt (5 Prozentpunkte).

- Kraftrollerfahrer haben einen deutlich überhöhten Anteil von Unfällen im Längsverkehr (16 Prozentpunkte). In der Zielgruppe ist die Grundgesamtheit mit 35 Fahrern allerdings sehr gering. 
- Die Fahrer der Altersgruppe, in welcher sich die Gruppe der Wiedereinsteiger befindet, weist einen erhöhten Anteil an Fahrunfällen auf (4 Prozentpunkte). Wird die Verteilung für die Unfälle betrachtet, die sowohl das Kriterium Schlechtwetterunfälle als auch das Alterskriterium für die Wiedereinsteiger erfüllen, zeigt sich im Vergleich zu den Schlechtwetterunfällen allein ein nochmals höherer Anteil an Fahrunfällen (8 Prozentpunkte). D. h., ein witterungsbedingt schlechter Fahrbahnzustand führt anteilsmäßig häufiger zu Fahrunfällen. Bei solchen Bedingungen ereignen sich für die Altersgruppe der Wiedereinsteiger anteilsmäßig öfter Unfälle als bei anderen Gruppen.

Außerorts sind ebenfalls Auffälligkeiten anzutreffen. Die Unfallhäufigkeit fällt deutlich geringer als innerorts, weshalb die Grundgesamtheit für die Anteilsberechnung bei den Zielgruppen eher niedrig ist.

- Jugendliche sind anteilsmäßig deutlich häufiger in Einbiegen/Kreuzen-Unfällen verwickelt (12 Prozentpunkte).

- Fahranfänger sind anteilsmäßig häufiger an Einbiegen/ Kreuzen-Unfällen bzw. Abbiegeunfällen beteiligt (4 bzw. 2 Prozentpunkte).

- Schlechtwetterunfälle weisen einen größeren Anteil an Fahrunfällen auf (9 Prozentpunkte).

- Frauen sind anteilsmäßig häufiger bei Einbiegen/ Kreuzen-Unfällen bzw. Abbiegeunfällen beteiligt (4 bzw. 3 Prozentpunkte).

- Kraftrollerfahrer sind insgesamt nur an fünf Unfällen beteiligt gewesen. In vier Fällen handelte es sich um Fahrunfälle.

- Die Altersgruppe der Wiedereinsteiger weist einen leicht erhöhten Anteil an Fahrunfällen und Unfällen im Längsverkehr auf (je 2 Prozentpunkte). Wird die Verteilung für die Unfälle betrachtet, die sowohl das Kriterium Schlechtwetterunfälle als auch das Alterskriterium für die Wiedereinsteiger erfüllen, zeigt sich im Vergleich zu den Schlechtwetterunfällen allein ein nochmals höherer Anteil an Fahrunfällen (6 Prozentpunkte).

Zusammenfassend wird für die Unfallauswertung der festgelegten Streckenabschnitte in Sachsen in Bezug auf Zahl und
Schwere der Unfälle von motorisierten Zweirädern folgende Punkte von hervorgehobener Bedeutung festgehalten:

\section{Innerorts:}

- dreifach so hohe Unfalldichte wie außerorts, hingegen deutlich geringere Unfallschwere (1 \% der Unfälle sind Unfälle mit Getöteten, 19 \% sind Unfälle mit schwerem Personenschaden, 58 \% sind Unfälle mit Personenschaden); die Unfälle fallen jedoch schwerer aus als die Innerortsunfälle aller Verkehrsteilnehmer

- Schwerpunkt bilden die Knotenpunkte (52 \% aller Unfälle) - durch paarweisen Vergleich der Strecken hoher vs. Strecken geringer Unfalldichte auffällig geworden: Unfälle an Knotenpunkten, Schlechtwetterunfälle, Unfälle mit Kraftrollerfahrern

- Unfallschwere: hoch bei EK-Unfällen sowie (Fahr-) Unfällen in Kurven

- Zielgruppe der Jugendlichen von besonderer Bedeutung (stellt $1 / 4$ aller unfallbeteiligten Fahrer von PTW, anteilsmäßig fast doppelt so häufig in Unfall verwickelt wie außerorts)

- ebenso sind Fahranfänger, Frauen und Kraftrollerfahrer innerorts anteilsmäßig häufiger an einem Unfall beteiligt als außerorts.

\section{Außerorts:}

- hohe Unfallschwere (5 \% der Unfälle sind Unfälle mit Getöteten, 48 \% sind Unfälle mit schwerem Personenschaden, $78 \%$ sind Unfälle mit Personenschaden) - Handlungsbedarf besteht vor allem für den Ausflugsund Freizeitverkehr: $38 \%$ der Unfälle passieren am Wochenende (ohne Freitag), 38 \% der Unfälle sind Alleinunfälle, 42 \% der Unfälle sind Fahrunfälle - Schwerpunkt bilden Unfälle in Kurven mit knapp der Hälfte aller Unfälle sowie Unfälle an Knotenpunkten und auf Strecken mit Längsneigung mit jeweils ungefähr 1/3 der Unfälle (Mehrfachauftreten möglich) - durch paarweisen Vergleich der Strecken hoher vs. Strecken geringer Unfalldichte auffällig geworden: Unfälle an Knotenpunkten, Schlechtwetterunfälle, Unfälle auf Strecken mit Längsneigung

- Unfallschwere: hoch bei (Fahr-)Unfällen in Kurven. 


\subsubsection{Unfallgeschehen und Straßeneigenschaften auf den besichtigten Streckenabschnitten}

Bei der Ortsbegehung ausgewählter Strecken waren bestimmte Straßeneigenschaften auf allen bzw. fast allen Abschnitten gleich ausgeprägt (z. B. Fahrbahnbelag Asphalt, Leitpfosten, keine Schutzplanken mit speziellem Motorradschutz wie bspw. Pfostenummantelung). Für diese Eigenschaften fand keine Gegenüberstellung der Abschnitte mit hoher und geringer Unfalldichte statt. Für die anderen aufgenommenen Straßeneigenschaften wurde für ein festgelegtes Ausprägungskriterium der Anteil der Abschnitte mit hoher dem Anteil der Abschnitte mit geringer Unfalldichte gegenübergestellt, um so mögliche Einflussgrößen der Straße auf das Unfallgeschehen von motorisierten Zweirädern zu ermitteln bzw. potentielle Einflussgrößen auszuschließen. Um den Einfluss von unterschiedlichen Abschnittslängen zu berücksichtigen, wurden die Anteile der Abschnittslängen bestimmt. Bei der Interpretation der Ergebnisse ist zu beachten, dass ein Anwachsen der Differenz zwischen den beiden berechneten Anteilen bei Eigenschaften mit linienhaftem Auftreten (z. B. Fahrbahnbreite) den möglichen Ein- fluss der Straßeneigenschaft stärker fundiert, während dies bei Eigenschaften mit punktuellem Auftreten (z. B. vorhandene Sichteinschränkung) eher gegen den angenommenen Einfluss spricht, da die Auftretenswahrscheinlichkeit einer punktuellen Eigenschaft mit der Abschnittslänge zunimmt.

\subsubsection{Außerorts}

Außerorts führte die Einteilung der besichtigen $A b$ schnitte in zwei Klassen anhand der Unfalldichte (vgl. Abschnitt 5.1.4) und die anschließende Auswertung der aufgenommenen Straßeneigenschaften zu folgenden Ergebnissen:

Demnach deutet sich auf Landstraßen für folgende Straßenmerkmale ein Zusammenhang mit dem Unfallgeschehen motorisierter Zweiräder an:

- Knotenpunktdichte

- Anzahl der Hochpunkte (Kuppen) auf einem Abschnitt, insbesondere vor oder in Kurven bzw. vor oder an Knoten (die prozentuale Differenz im Diagramm würde

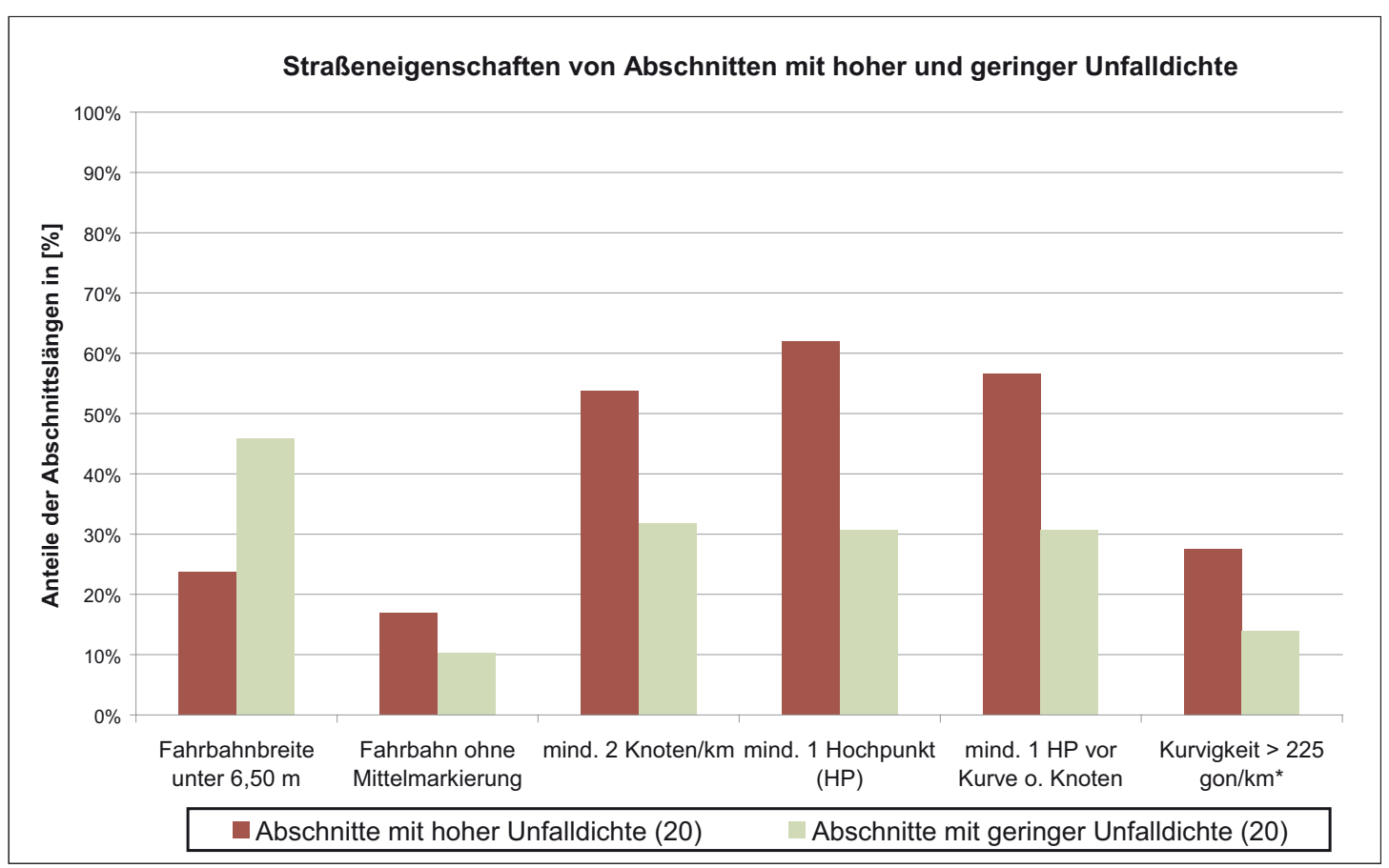

Abbildung 5-9: Paarweiser Vergleich der Anteile der Abschnittslängen mit bestimmten Straßeneigenschaften außerorts (1) 
noch erheblich stärker ausfallen, wenn die Anzahl der Kuppen pro Abschnitt betrachtet wird)

- bestehende Sichteinschränkungen (z. B. Brückenwiderlager, Dammlage, Bäume, Häuser, Kuppen)

- Gefälle-/Steigungsstrecken

- hohe Kurvigkeit.

Der aus dem Diagramm abzuleitende Einfluss für die Merkmale „zulässige Höchstgeschwindigkeit von mehr als 70 km/h“ sowie „Steigung/Gefälle nach Z 108/110 StVO“ ist eher gering. Im Gegensatz dazu fällt der Einfluss bei den Merkmalen Knoten, Hochpunkte sowie Kurvigkeit sehr deutlich aus. Es wurden nur die Strecken mit Längsneigung aufgenommen, die mit entsprechenden Verkehrszeichen beschildert sind, da digitale Streckendaten in der Straßendatenbank wie im Fall der Kurvigkeit nicht vorlagen. Als Grenzwert für Abschnitte mit hoher Kurvigkeit wurden 225 gon/km gewählt. Der Wert entspricht dem Grenzwert nach HBS [FGSV 2005] für den
Bereich mit der höchsten Kurvigkeit, der beim Verfahren zur Bestimmung der Qualität des Verkehrsablaufes auf zweistreifigen Landstraßen verwendet wird.

Keine Auffälligkeiten wurden für folgende Straßeneigenschaften festgestellt, die ebenfalls auf einen Zusammenhang untersucht wurden:

- geringe Fahrbahnbreite (kleiner 6,50 m)

- Fahrbahnen ohne Mittelmarkierung

- Mängel im Straßenzustand (Spurrinnen, Ausbrüche, Flickstellen etc.)

- Wildwechsel/Tiere nach Z 140 und Z 142 StVO

- per Z 276 StVO angeordnetes Überholverbot

- Hindernisse im Seitenraum.

Bei den Straßeneigenschaften, die anhand der aufgestellten Verkehrszeichen erfasst wurden, sind Ungenauigkeiten zu berücksichtigen. Diese entstehen durch eine fehlende Aufnahme von Verkehrsschildern, die vor

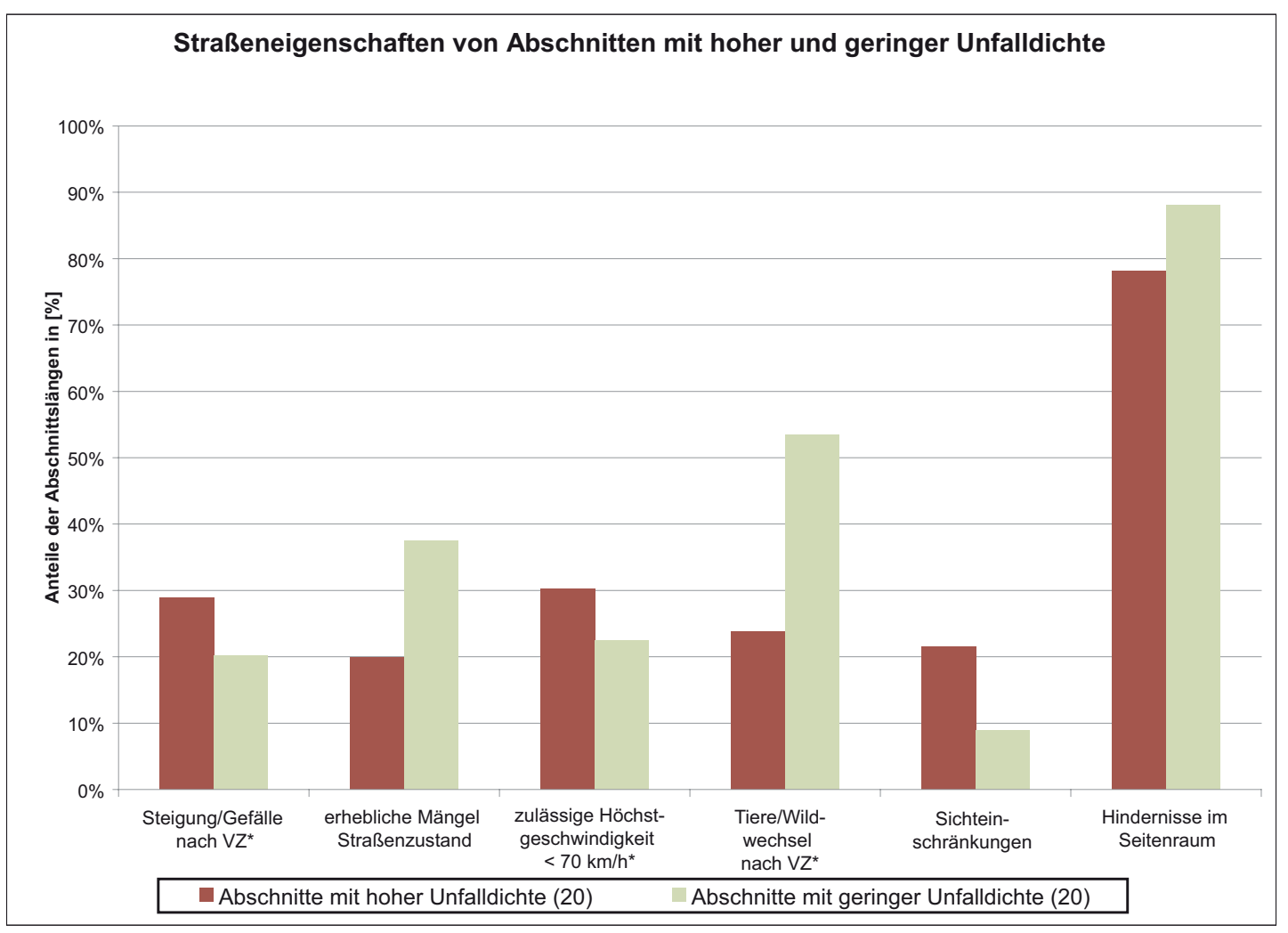

Abbildung 5-10: Paarweiser Vergleich der Anteile der Abschnittslängen mit bestimmten Straßeneigenschaften außerorts (2) 
Beginn des Abschnittes aufgestellt sind, aber auch für diesen gelten, sowie dadurch, dass die Verkehrszeichen oft nicht für den gesamten Abschnitt, sondern nur einen Teilbereich gelten.

Das Merkmal „Hindernisse im Seitenraum“ ist weniger für die Unfallauffälligkeit entscheidend, sondern eher für die Untersuchung einer Auffälligkeit besonders schwerer Folgen bei bestimmten Ausprägungsarten. Auch aus der zusätzlichen Untergliederung des Merkmales in:

(1) kein Hindernis

(2) vereinzelte Hindernisse

(3) mindestens $25 \%$ und weniger als $50 \%$ der Ab-schnittslänge führen durch Waldgebiet oder es befinden sich Einzelhindernisse in regelmäßigem Abstand im Seitenraum (z. B. Bäume)

(4) mindestens $50 \%$ der Abschnittslänge führen durch Waldgebiet resultierte keine Auffälligkeit. Abschnitte mit hoher Unfalldichte weisen nicht häufiger das Kriterium (3) oder (4) auf als die Abschnitte mit geringer Unfalldichte.

Der Einfluss von Knotenpunkten und Strecken mit (erhöhter) Längsneigung wird durch die Unfallauswertung der besichtigten Strecken belegt. Dort kann ein deutlich erhöhter Anteil von Unfällen mit der Charakteristik Knotenpunkt sowie Steigungs- oder Gefällestrecke auf den Abschnitten mit hoher Unfalldichte gefunden werden (Abbildung 5-11):

Dass der Anteil der Unfälle in Kurven auf unfallauffälligen Strecken nicht deutlich größer als bei den nichtauffälligen Abschnitten ist, lässt sich dadurch erklären, dass nicht die Kurve selbst, sondern die Kurvigkeit als Summe der Richtungsänderungen pro Kilometer den Einflussfaktor darstellt. Um die Übertragbarkeit der Aussagen zu prüfen, wurden in den nachfolgenden beiden Tabellen die Anteile

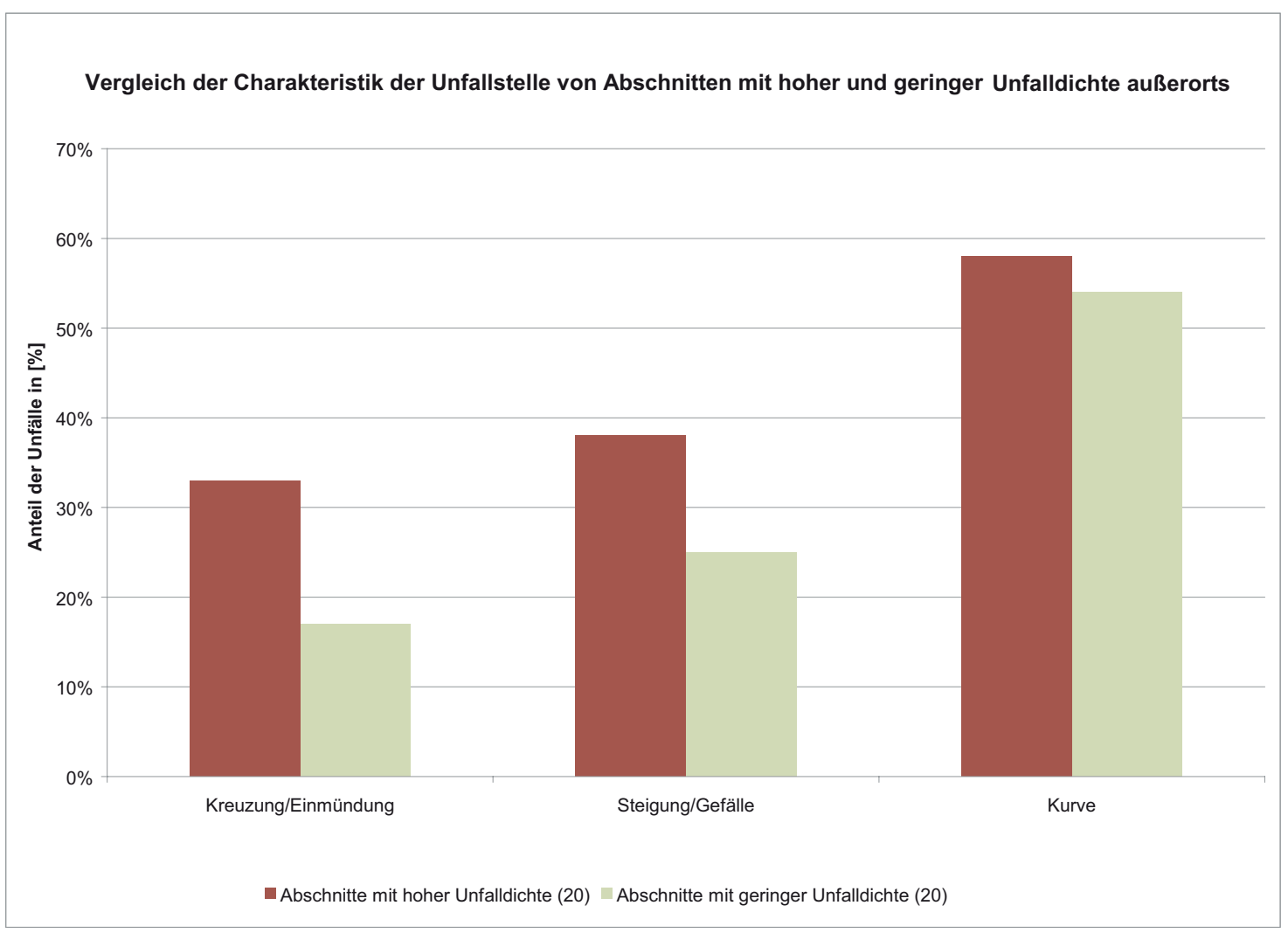

Abbildung 5-11: Anteile der Unfälle an bestimmten Örtlichkeiten für die besichtigen Streckenabschnitte 
der Unfälle an bestimmten Örtlichkeiten von den besichtigten Strecken und die jeweiligen Anteile von allen definierten Streckenabschnitten in Sachsen gegenübergestellt:

\begin{tabular}{|l|c|c|}
\hline & $\begin{array}{l}\text { Abschnitte mit } \\
\text { geringer UD }\end{array}$ & $\begin{array}{l}\text { Abschnitte mit } \\
\text { hoher UD }\end{array}$ \\
\hline $\begin{array}{l}\text { besichtigte } A b- \\
\text { schnitte alle U }\end{array}$ & $25 \%$ & $38 \%$ \\
\hline $\begin{array}{l}\text { besichtigte Ab- } \\
\text { schnitte U (P) }\end{array}$ & $13 \%$ & $37 \%$ \\
\hline 219 Abschnitte alle U & $26 \%$ & $35 \%$ \\
\hline 219 Abschnitte U (P) & $24 \%$ & $35 \%$ \\
\hline
\end{tabular}

Tabelle 5-7: Vergleich der Unfallanteile außerorts mit „Steigung“ oder "Gefälle“ als Charakteristik der Unfallstelle

\begin{tabular}{|l|c|c|}
\hline & $\begin{array}{l}\text { Abschnitte mit } \\
\text { geringer UD }\end{array}$ & $\begin{array}{l}\text { Abschnitte mit } \\
\text { hoher UD }\end{array}$ \\
\hline $\begin{array}{l}\text { besichtigte Ab- } \\
\text { schnitte alle U }\end{array}$ & $54 \%$ & $58 \%$ \\
\hline $\begin{array}{l}\text { besichtigte } A b- \\
\text { schnitte U (P) }\end{array}$ & $56 \%$ & $60 \%$ \\
\hline 219 Abschnitte alle U & $48 \%$ & $49 \%$ \\
\hline 219 Abschnitte U (P) & $48 \%$ & $54 \%$ \\
\hline
\end{tabular}

Tabelle 5-8: Vergleich der Unfallanteile außerorts mit „Kurve“ als Charakteristik der Unfallstelle
Es zeigen sich insgesamt gute Übereinstimmungen. Die Abweichung des Anteiles der Unfälle mit Charakteristik „Steigung/Gefälle“ bei den besichtigten Abschnitten mit geringer Unfalldichte (13\%) ist mit der geringen absoluten Unfallanzahl erklärbar (24). Der grundsätzlich höhere Anteil der Unfälle in Kurven für die besichtigten Streckenabschnitte lässt auf eine vermehrte Auswahl von kurvenreichen Strecken mit häufigen Radien- und Richtungswechseln schließen.

Abschließend wird der Einfluss der Knotenpunkte auf das Unfallgeschehen analysiert. Hierfür wurde eine lineare Regressionsanalyse durchgeführt, in welcher der Zusammenhang von Unfalldichte und Knotenpunktdichte für 34 der 40 besichtigen Außerortsabschnitte untersucht wurde. Sechs Abschnitte, auf denen zwar Unfälle aber keine Knotenpunkte vorhanden sind, wurden nicht betrachtet, da in diesem Fall der Einfluss von Knoten auf das Unfallgeschehen vollständig ausgeschlossen werden kann. Dies hat als weitere Annahme für die Regressionsanalyse zur Folge, dass der Verlauf der Regressionsgerade durch den Nullpunkt festgelegt wird.

Das Ergebnis zeigt mit einem Korrelationskoeffizienten $r$ $=0,83$, dass ein Zusammenhang gegeben ist. Dieser wird

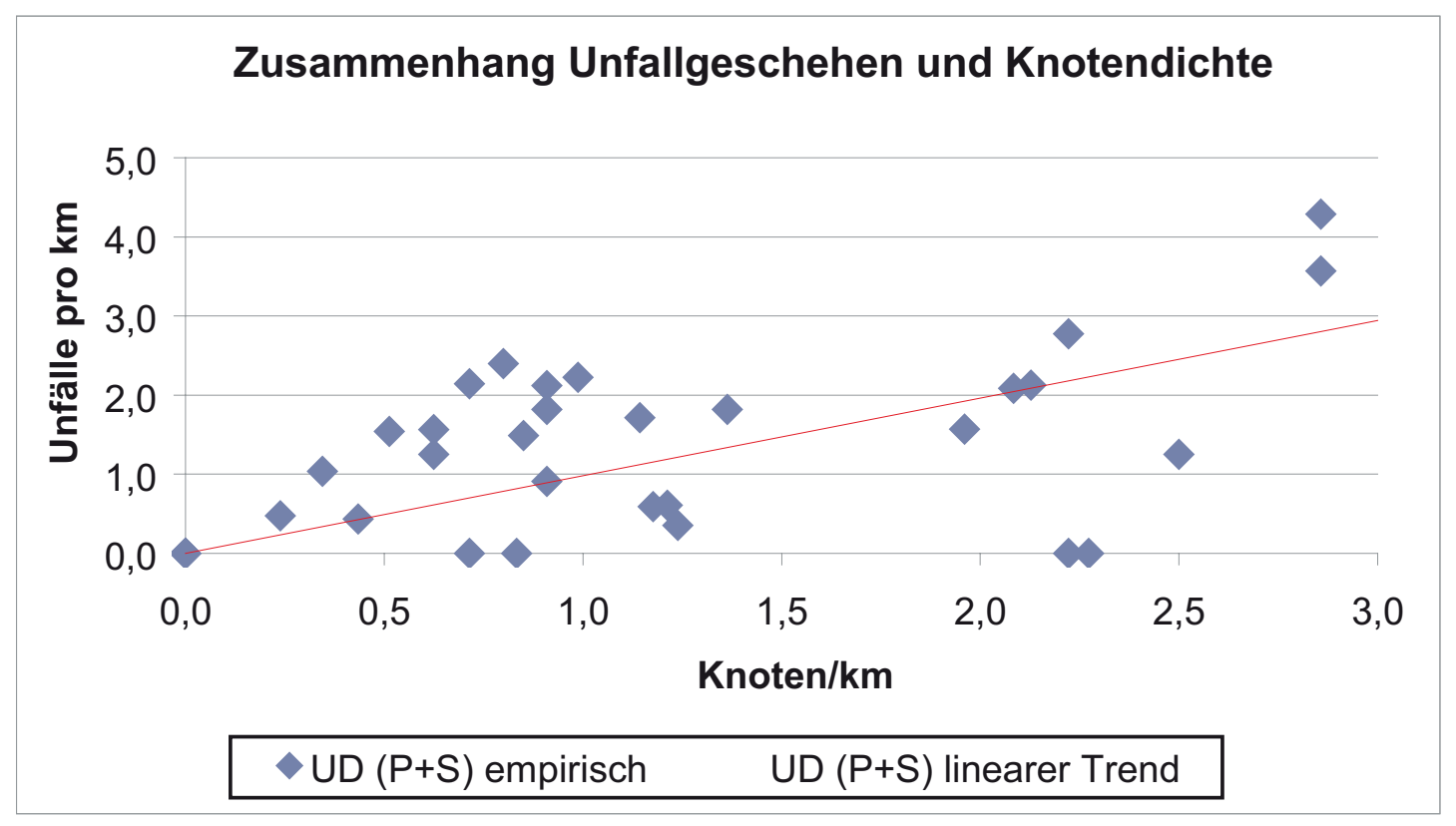

Abbildung 5-12: Zusammenhang Unfalldichte und Knotendichte auf den besichtigen Strecken außerorts 
in der Realität weder linear sein, noch ist die Unfalldichte allein von der Knotendichte abhängig (was auch die Herausnahme der sechs Abschnitte ohne Knoten zeigt), die Häufigkeit von Knotenpunkten hat aber einen wesentlichen Einfluss auf die Unfalldichte.

\subsubsection{Innerorts}

Bei den Streckenabschnitten innerorts wurden aufgrund der unterschiedlichen Charakteristik andere Kriterien als außerorts für die Auswertung des Einflusses von Straßeneigenschaften herangezogen. Die Ergebnisse werden in der Abbildung 5-13 deutlich.

Daraus lässt sich ein deutlicher Einfluss von Mängeln im Straßenzustand sowie Straßenbahnverkehr mit straßenbündiger Führung ableiten. Das Ankündigen von Straßenschäden per Verkehrszeichen ist vor allem auf den Abschnitten mit den erfassten Straßenmängeln zu finden. Insofern stärkt das Resultat in dem Merkmal das Ergebnis in Bezug auf die Defizite im Straßenzustand. Einen weniger ausgeprägten Einfluss weisen den Straßenverlauf kreuzende Gleisanlagen sowie Straßen mit zweistreifigen Richtungsfahrbahnen oder baulicher Mitteltrennung auf. Letztgenanntes Merkmal tritt in Kombination mit zweistreifigen Richtungsfahrbahnen auf.

Der hohe Anteil von Abschnitten mit kreuzenden Gleisanlagen bzw. mit Straßenbahnverkehr auf der Fahrbahn ergibt sich aus der Tatsache, dass sich ein erheblicher Teil der besichtigten Innerortsabschnitte im Großraum Dresden befindet. Dort ist auf den Hauptverkehrsstraßen entsprechend häufig Straßenbahnverkehr anzutreffen. Ob das Straßenmerkmal an sich unfallbegünstigend wirkt, oder nur in Zusammenhang mit anderen Eigenschaften steht, die sich auf das Unfallgeschehen auswirken, muss im folgenden Abschnitt bei der Detailanalyse der einzelnen Unfallkonstellationen überprüft werden. Eine unfallbegünstigende Wirkung kann sich z. B. in der Form darstellen, dass Gleisanlagen an Knoten die Komplexität von Kreuzungs- und Abbiegevorgängen verstärken sowie die Übersichtlichkeit einschränken und auf der freien Strecke wie an Knoten zu einer Veränderung der Griffigkeit - besonders in Kombination mit Nässe - für das passierende motorisierte Zweirad führen.

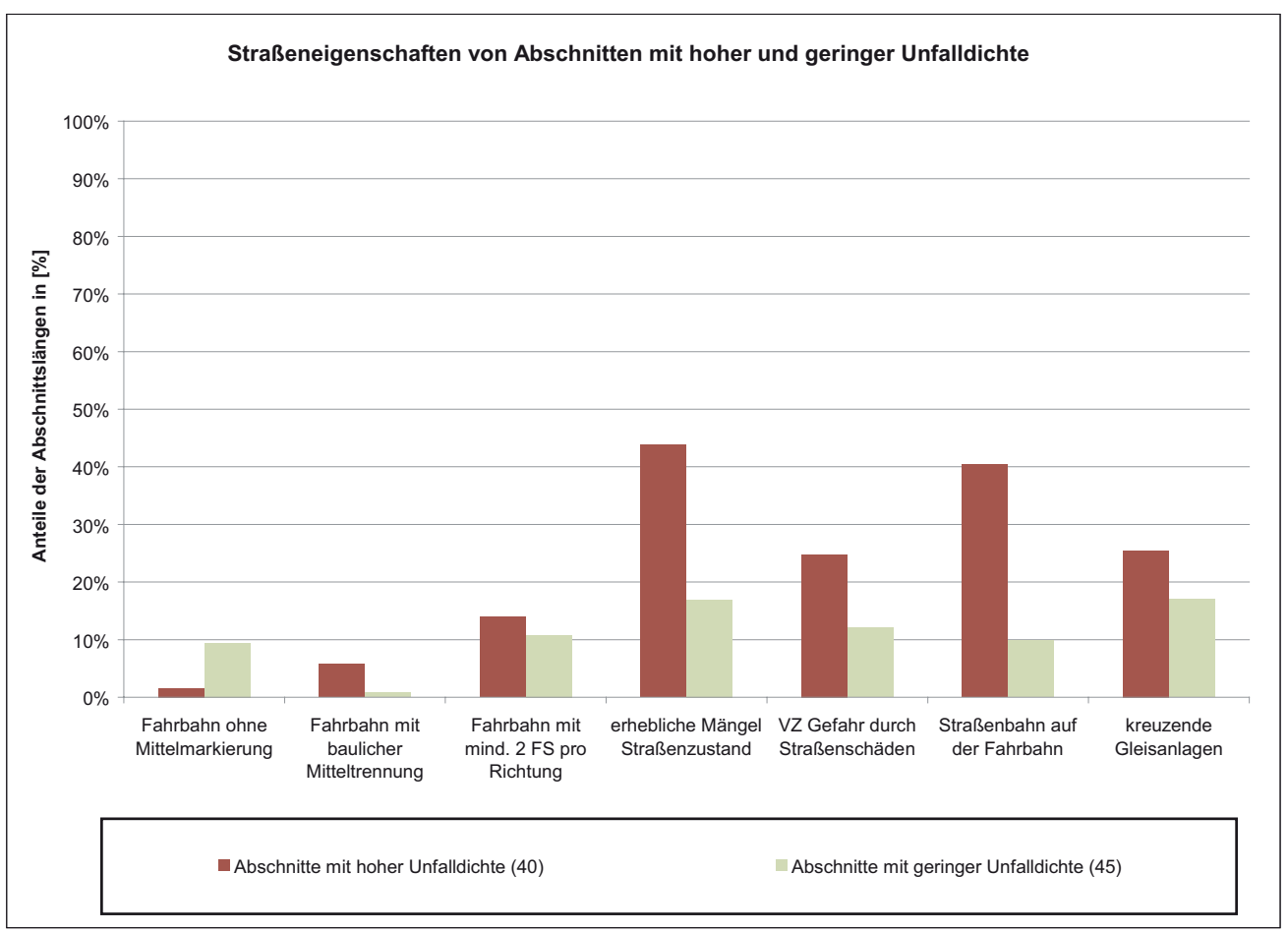

Abbildung 5-13: Paarweiser Vergleich der Anteile der Abschnittslängen mit bestimmten Straßeneigenschaften innerorts (1) 
Die Ergebnisse für weitere Merkmale, welche für die Innerortsabschnitte untersucht wurden, sind in Abbildung 5-14 ersichtlich.

Der als Bordstein ausgeprägte Fahrbahnrand scheint auch einen Einfluss auszuüben, ein kausaler Zusammenhang ist in dem Fall jedoch nicht zu sehen. Vielmehr ist ein indirekter Einfluss in sofern zu vermuten, dass der Bordstein anderer Straßeneigenschaften mit repräsentiert, die wiederum unfallbegünstigend sind.

Weiterhin lässt sich noch von Streckenabschnitten mit zulässiger Höchstgeschwindigkeit über 50 km/h sowie von Abschnitten mit lückenhafter Bebauung ein Einfluss auf das Unfallgeschehen erkennen, welcher jedoch sehr schwach ausgeprägt ist. Dies könnte auf höhere Unfalldichten in Straßen im Randbereich der verdichteten Bebauung, also Ausfallstraßen oder Ortsdurchfahrten-ähnliche Typen hinweisen.
Die Untersuchung des Zusammenhanges von Unfalldichte und Knotenpunktdichte erfolgte analog dem Vorgehen auf Landstraßen und führt zu einem ähnlichen Resultat (Abbildung 5-15).

Es wurden 78 der 85 Innerortsabschnitte ausgewertet, die sieben herausgenommenen Abschnitte verzeichneten Unfälle, ohne Knotenpunkte aufzuweisen. Mit der Annahme, dass die Regressionsgerade durch den Nullpunkt verlaufen soll, ergibt sich ein Korrelationskoeffizient von 0,82. Ein Einfluss der Knotenpunktdichte auf die Höhe der Unfalldichte ist demnach gegeben. Offen bleibt bei dieser Betrachtung die Frage nach dem Grund der Zunahme von Unfällen auf einem Abschnitt bei Zunahme der Knotenzahl, d.h. danach, welche Eigenschaften eines Knotenpunktes sich besonders auf das Unfallgeschehen auswirken.

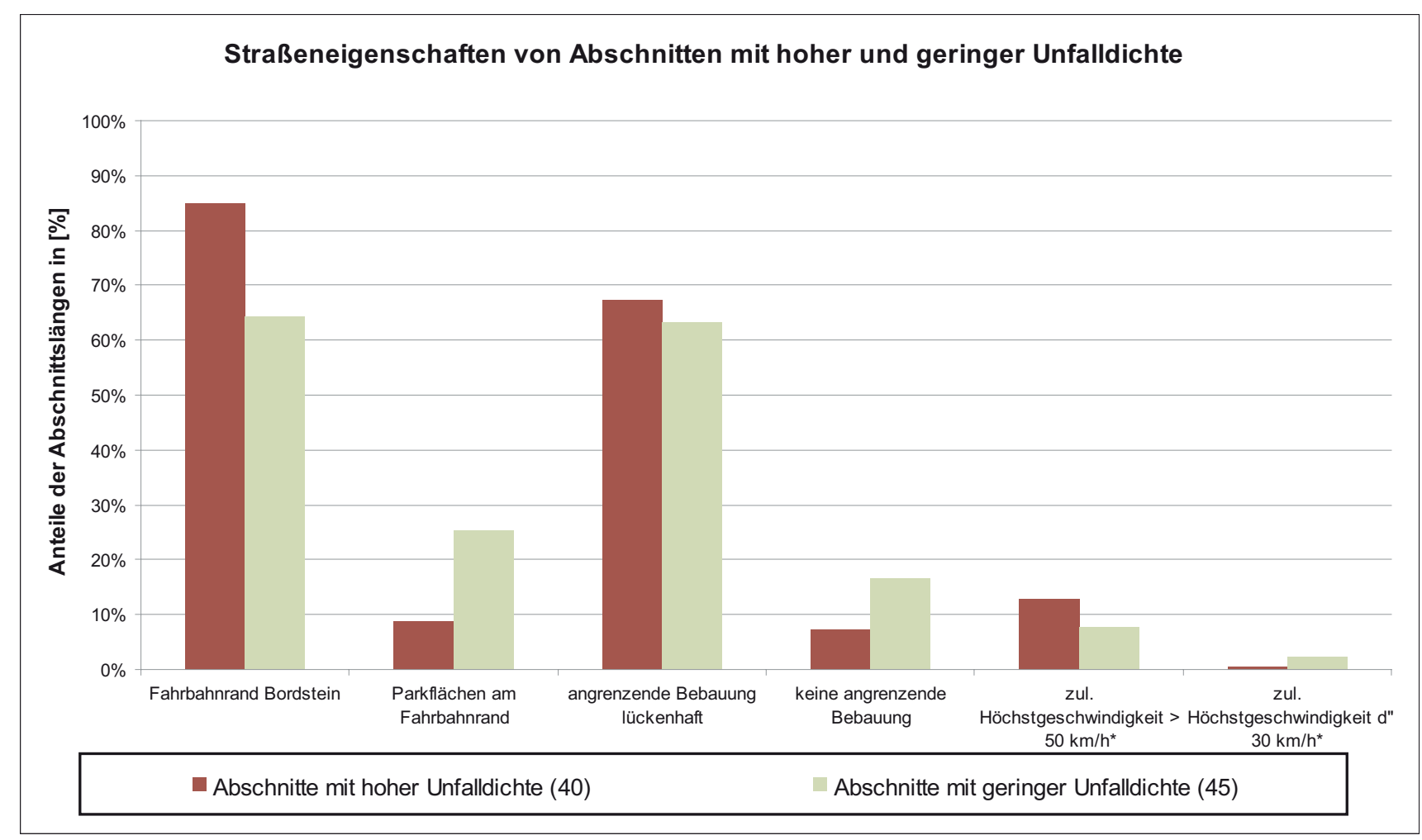

Abbildung 5-14: Paarweiser Vergleich der Anteile der Abschnittslängen mit bestimmten Straßeneigenschaften innerorts (2) 


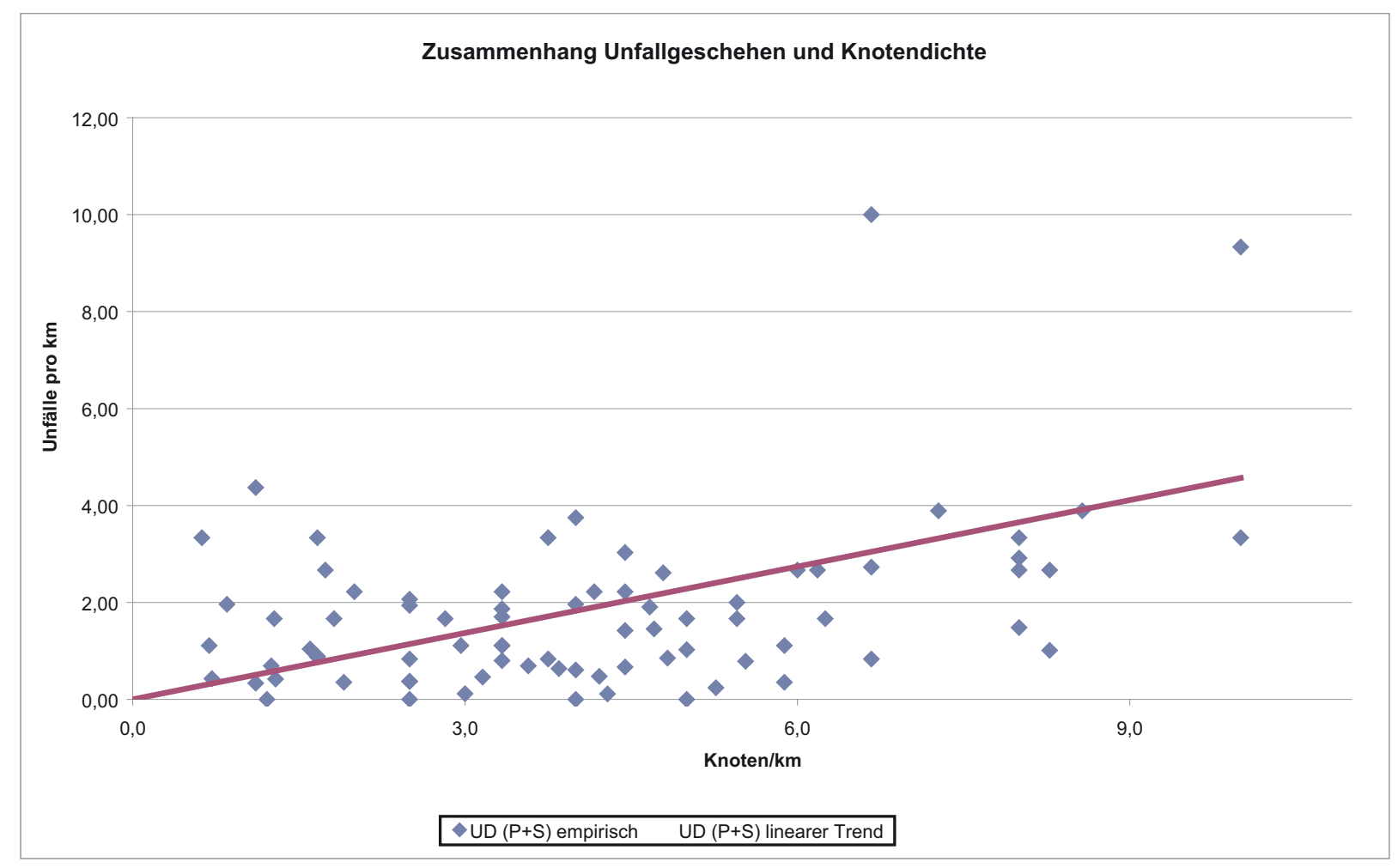

Abbildung 5-15: Zusammenhang zwischen Unfalldichte und Knotendichte auf den besichtigten Abschnitten innerorts

\subsubsection{Detailanalyse typischer Unfallkonstellationen}

Die Betrachtung typischer Unfallkonstellationen für das Unfallgeschehen motorisierter Zweiräder bildet die detaillierteste Stufe der vorliegenden Untersuchung. Ziel ist es, zum einen über diese Konstellationen die Verknüpfung zwischen den beiden Fachbereichen (Straße und Fahrzeug) zu realisieren, und zum anderen für typische, klar abgrenzbare Unfallsituationen Aussagen über charakeristische Ausprägungen der Unfallmerkmale sowie über unfallbegünstigende Straßen- oder Fahrzeugeigenschaften zu treffen. Die Auswertung des Unfallgeschehens und der Straßeneigenschaften erfolgt in diesem Abschnitt für jede einzelne Unfallkonstellation. Bei der Zusammenführung der Daten im folgenden Kapitel können nur fachübergreifende Ergebnisse für die Konstellationen ermittelt werden, bei denen eine ausreichende Schnittmenge von Daten vorgefunden wurde. Für die Konstellationen, bei denen dies nicht der Fall war, wurde über eine Änderung oder Zusammenfassung von Unfallkonstellationen eine ausreichende Schnittmenge angestrebt.
Die Bildung der Konstellationen erfolgt in einem ersten Schritt anhand der Unfallmerkmale Ortslage, Charakteristik der Unfallstelle und Unfalltyp (Abbildung 5-16).

Für die fett markierten 12 Unfallkollektive innerorts wurde eine Detailanalyse hinsichtlich weiterer wichtiger Unfallmerkmale sowie der Ausprägung interessanter Straßeneigenschaften durchgeführt. Außerorts ist die Größe der Kollektive geringer. Es wurden nur Kollektive mit einer Unfallzahl größer 5 analysiert. Aus Abbildung 5-17 resultieren sieben auszuwertende Fälle.

Auf Basis dieser 19 Fälle (vgl. Anlage 06) erfolgt ein weiteres Einschärfen der Kollektive anhand der Unfallart und in Einzelfällen zusätzlich anhand der Unfallschwere und des Kriteriums Alleinunfall. Durch sinnvolles Zusammenfassen resultieren schließlich 10 typische Unfallkonstellationen, für die ebenso wie für die 19 genannten Fälle eine Detailanalyse der Unfallmerkmale sowie die Auswertung 
bestimmter Straßeneigenschaften durchgeführt wurde. $\mathrm{Da}$ außerorts mit diesem Vorgehen nur zwei typische Konstellationen entstanden, wurde zusätzlich sowohl für alle Überholunfälle in Außerortslage als auch alle $\mathrm{Au}$ Berortsunfälle mit der Charakteristik Steigung/Gefälle in der VUA dieselben Auswertungen durchgeführt. Dabei kristallisierte sich jedoch keine weitere typische Unfallkonstellation heraus.

Die Auswertung des Unfallherganges der Einzelunfälle mit Hilfe der polizeilichen Unfalltexte brachte zusätzliche Erkenntnisse, welcher typische Unfallablauf den einzelnen Unfallkonstellationen in der Mehrheit der Fälle zugrunde liegt. Dies führt zu nachfolgendem Schaubild (Abbildung 5-18), in dem für jede der 10 Konstellationen der typische Unfallhergang abgebildet ist:

Für die Konstellation 4 sind zwei verschiedene Schau- bilder dargestellt, da sich für Einmündung und Kreuzung erkennbare Unterschiede im typischen Unfallhergang ergaben. Gemeinsamkeiten im Unfallgeschehen sind dennoch zu einem großen Teil gegeben, weshalb insgesamt nur eine Konstellation gebildet wurde. Die Detailauswertungen erfolgen sowohl für die gesamte Unfallkonstellation als auch für Kreuzung und Einmündung getrennt.

Die Konstellationen mit Unfällen ohne Angabe einer charakteristischen Örtlichkeit in der EUSka-Datenbank enthalten Unfälle, die sich auf der freien Strecke ohne besondere Charakteristik ereignet haben. Es ist jedoch anzunehmen, dass darin auch Unfälle eingeschlossen sind, bei deren Aufnahme eine vorhandene Charakteristik nicht in der VUA festgehalten wurde. Die Unfälle ohne Charakteristik werden bei ausreichend hoher Fallzahl entweder im Fall von wesentlichen Unterschieden zu den anderen

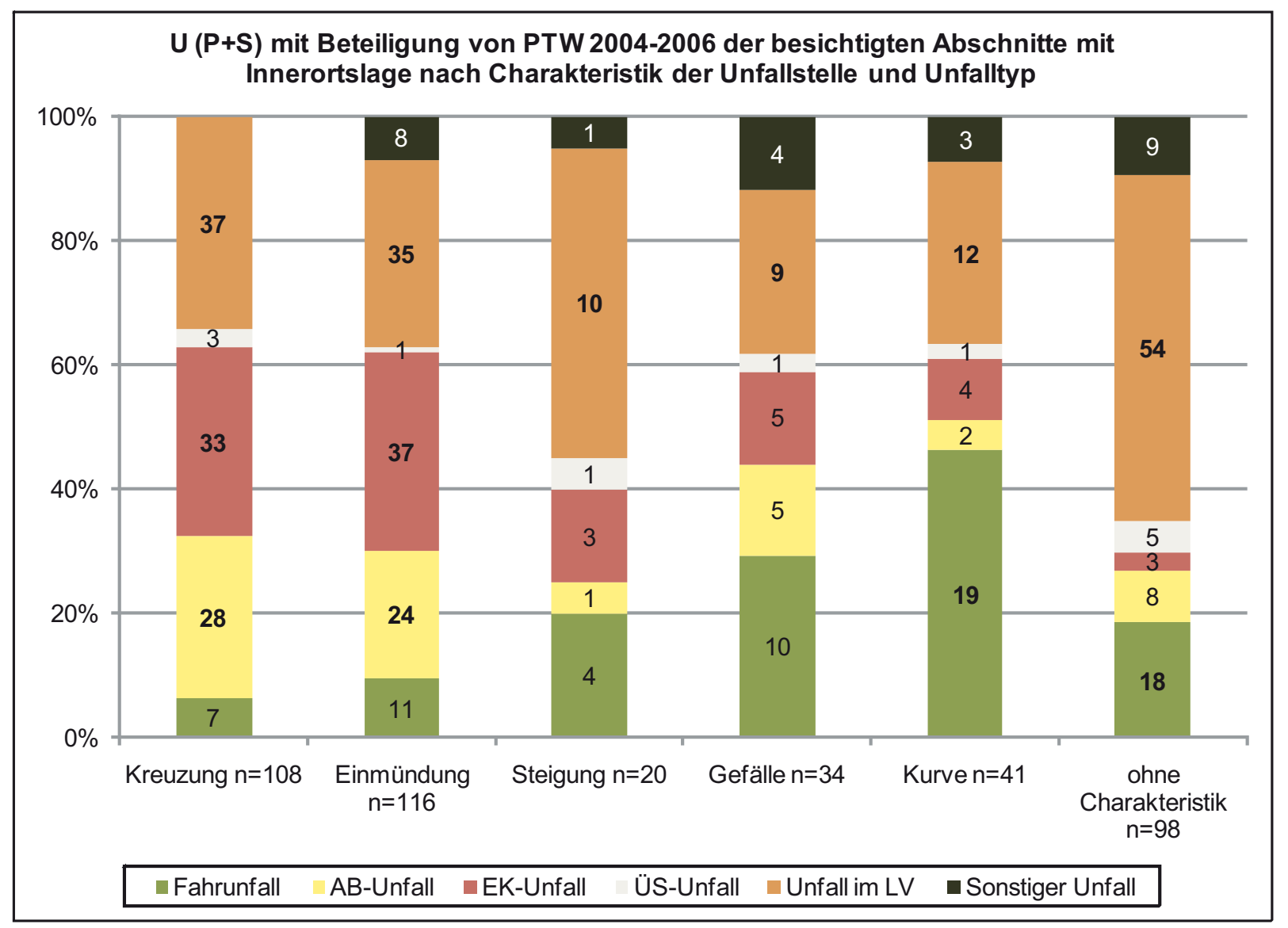

Abbildung 5-16: Verteilung der Unfälle auf den besichtigten Innerortsabschnitten nach Charakteristik der Unfallstelle und Unfalltyp 
Konstellationen in einer eigenen Unfallkonstellation untersucht oder im gegenteiligen Fall mit den Unfällen einer ähnlichen Konstellation zusammengefasst.

In Anlage 07 sind wichtige Kenngrößen (Unfallschwere, Hauptverursacher PTW, Unfallanteil am Wochenende) der Unfallkonstellationen auf Basis der Unfälle auf den besichtigten Streckenabschnitten und der Unfallkonstellationen auf Basis der Unfälle aller festgelegten Streckenabschnitte in Sachsen gegenübergestellt. Es zeigen sich nur geringe Abweichungen, so dass trotz der teilweise geringen Fallzahlen von einer repräsentativen Abbildung des Unfallgeschehens ausgegangen werden kann. Die Ergebnisse zu den zehn Unfallkonstellationen sind im zusammenführenden Kapitel 9 beschrieben. Alle Einzelergebnisse befinden sich für jede Unfallkonstellation detailliert in Anlage 08.

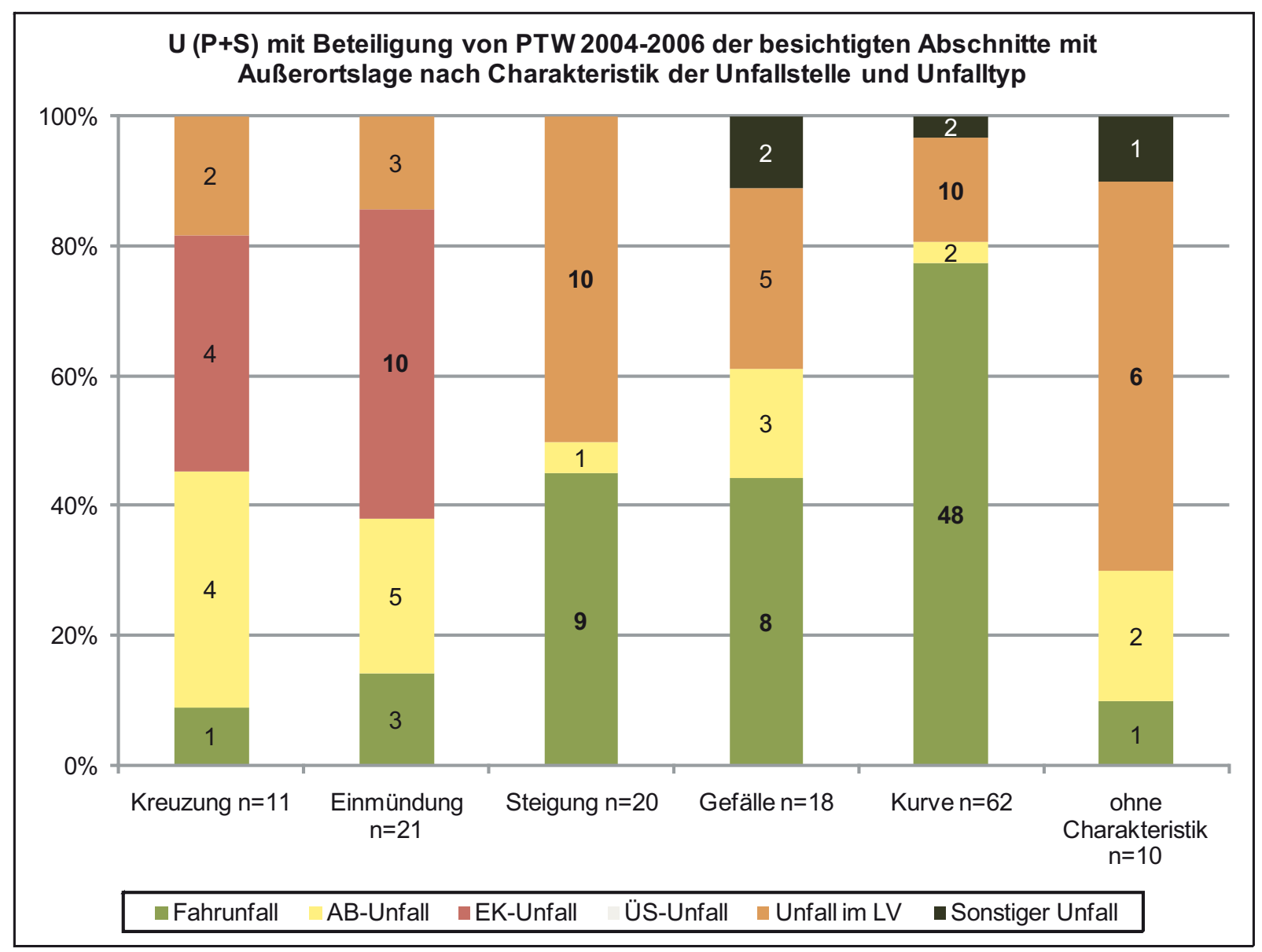

Abbildung 5-17: Verteilung der Unfälle auf den besichtigten Außerortsabschnitten nach Charakteristik der Unfallstelle und Unfalltyp 


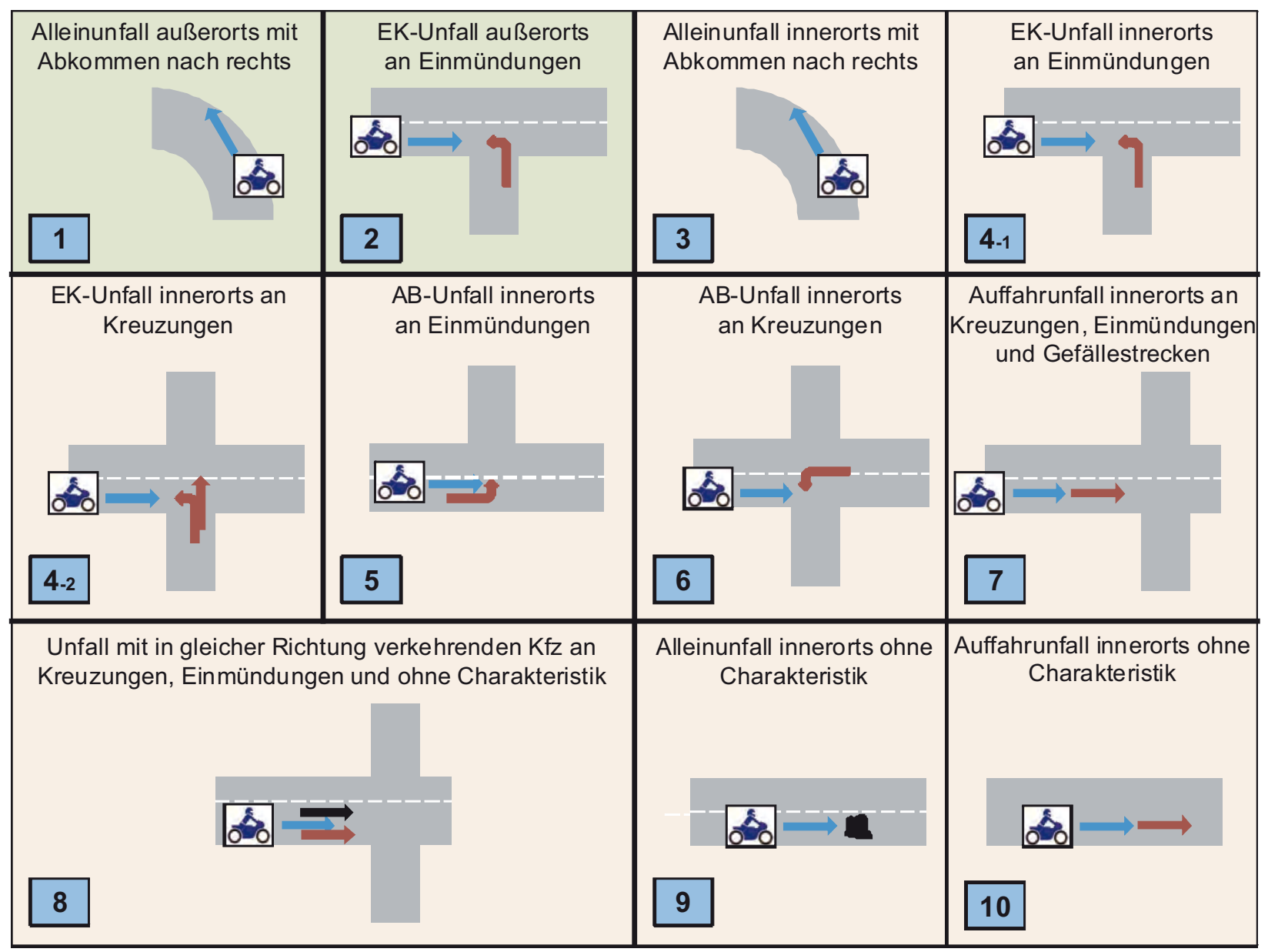

Abbildung 5-18: Typischer Unfallhergang der 10 definierten Unfallkonstellationen 


\section{Unfallgeschehen und Fahrzeugtechnik}

Zur Ermittlung der Faktoren, die aufgrund der Fahrzeugtechnik und/oder des Motorradfahrers selbst zu bestimmten Verletzungen führen oder diese verhindern, wurden drei unabhängige Stichproben erhoben, die jeweils, bedingt durch die grundsätzlich unterschiedliche Art und Weise der Erhebung, verschiedene Populationen abbilden. Die Datenbasen unterscheiden sich wie folgt:

\section{Datenbasis des Statistischen Bundesamtes:}

In der Verkehrsunfallstatistik des Statistischen Bundesamtes werden alle von der Polizei aufgenommenen Unfälle geführt und ermöglichen so durch eine großen Fallzahl statistisch begründete Ergebnisse aufzustellen. Nachteile dieser Datenbasis sind ihre geringe In- formationstiefe pro Einzelfall und eine schwer ermittelbare Dunkelziffer an Unfällen, die von der Polizei nicht erfasst werden. Durch eine Sonderauswertung wird die Berechnung der Korrelation zwischen dem Hubraum, der Fahrzeugleistung oder dem Leistungsgewicht ermöglicht. Detaillierte technische Eigenschaften können damit nicht berücksichtigt werden, da sie in der Datenbank nicht verfügbar sind. Die Verletzungsschwere wird in "unverletzt“, „leichtverletzt“, "schwerverletzt" und "getötet" unterschieden, wobei der Grenzwert zwischen „leichtverletzt“ und „schwerverletzt" durch die Aufenthaltsdauer im Krankenhaus von 24 Stunden unterscheidet. Diese Einteilung lässt keine Rückschlüsse auf den verletzten Körperteil oder eine differenzierte Betrachtung der Schwere der Verletzung zu.

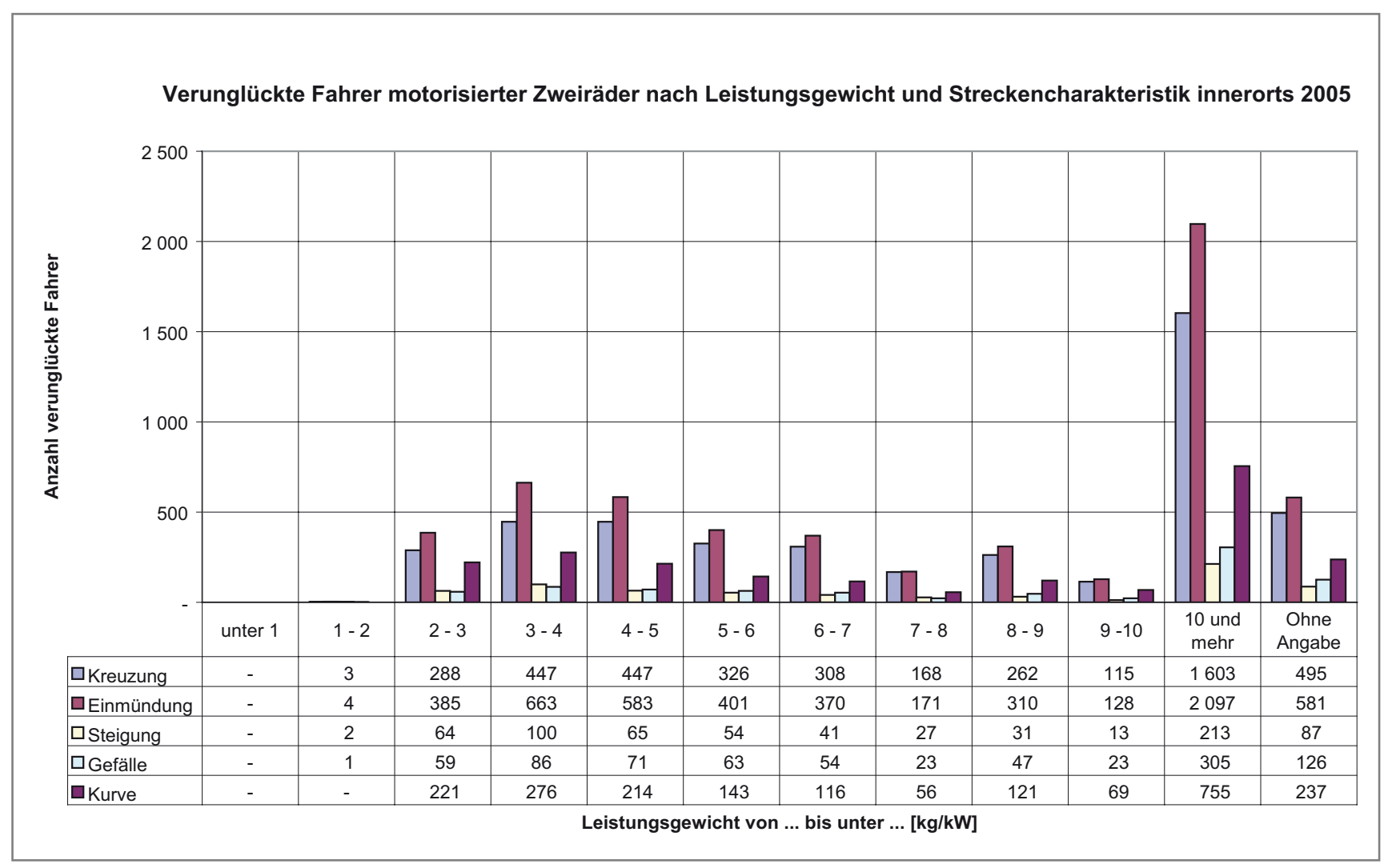

Abbildung 6-1: Zahl der Unfälle igo nach Leistungsgewicht und Charakteristik der Unfallstelle 


\section{Datenbasis des Versicherungswirtschaft (GDV):}

Die Unfallforschung der Versicherungswirtschaft führt eine tief detaillierte Datenbasis, die unter bestimmten Gesichtspunkten aus den einzelnen Schadensakten der versicherten Personen gespeist wird. Mit 1.304 Einzeldatensätzen steht eine umfangreiche Datenbasis zu Verfügung, die differenzierte und statistisch signifikante Auswertungen ermöglicht. Pro Datensatz sind bis zu 141 Merkmale aufgeführt. Die Erhebung der Datenbasis ist jedoch sehr gerichtet. Berücksichtigt wurden ausschließlich Motorradunfälle über einem Gesamtschaden von $15.000 €$ und zudem nur Unfälle, die der Versicherung gemeldet wurden. Somit werden insbesondere Alleinunfälle ohne Haftpflichtschaden bei Motorrädern ohne Vollkaskoversicherung nicht erfasst. Bei allen Aussagen zu Unfallhäufigkeiten sind daher Angaben auf dieser Datenbasis nicht repräsentativ.

\section{Datenbasis der Umfrage der TU-Berlin:}

In der Umfrage vom 19.01.2007 bis zum 18.02.2007 unter den Lesern der Zeitschrift „MOTORRAD“ wurden 6.864 Datensätze mit je 79 Antworten mit Hilfe eines Onlinefragebogens erhoben. Neben der Motorradfahrereinstellung und den Verkehrsverstößen wurden Angaben zu den erlittenen Motorradunfällen gemacht. So kann eine Korrelation zwischen Einstellung zum Motorradfahren im Straßenverkehr, Straßenverkehrsregelmissachtung und den Verletzungen durch einen Unfall erstellt werden.

Nachteil dieser Erhebung ist jedoch, dass nur Leser der Zeitschrift MOTORRAD an der Umfrage teilgenommen haben und ein Großteil davon Sportmaschinenfahrer sind. Auch wurde hauptsächlich über leichte Verletzungen berichtet, es ist kein Fall von getöteten Personen enthalten.

Verunglückte Fahrer motorisierter Zweiräder nach Leistungsgewicht und Streckencharakteristik außerorts ohne BAB 2005

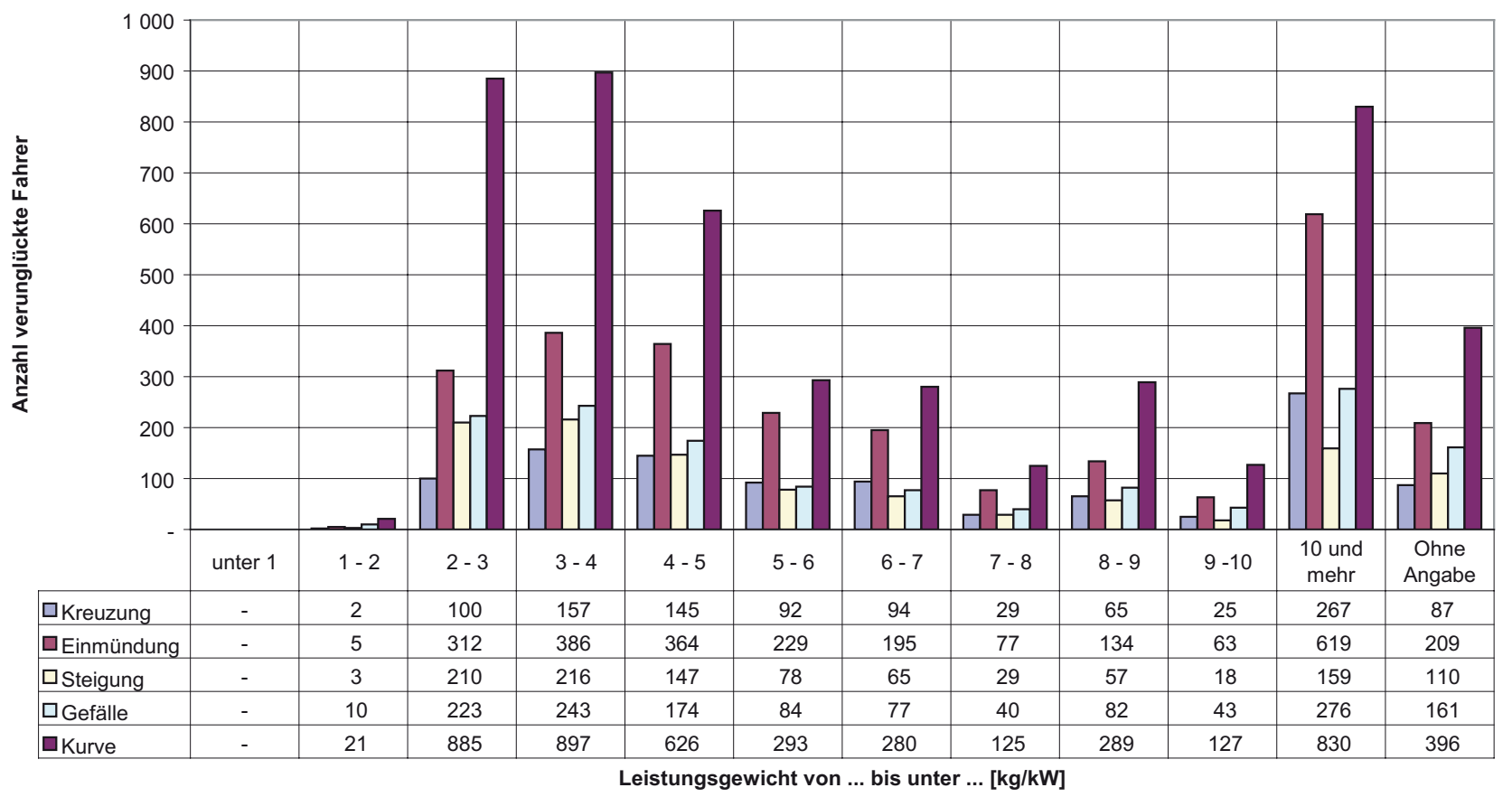

Abbildung 6-2: Zahl der Unfälle ago nach Leistungsgewicht und Charakteristik der Unfallstelle 


\subsection{Auswertung der Daten des Statisti- schen Bundesamtes}

In der Bundesstatistik sind zwar viele, dafür aber nicht sehr ausführlich beschriebene Unfälle geführt. Eine Sonderauswertung bezüglich fahrzeugtechnischer Eigenschaften soll eine Deutung technischer Eigenschaften mit einer großen Fallzahl ermöglichen. Da eine genaue Beschreibung der in den Unfall verwickelten Fahrzeuge nicht erfolgen kann, wird an dieser Stelle nur auf den Einfluss des Leistungsgewichts auf die Unfallhäufigkeit und Verletzungsschwere Bezug genommen.

Betrachtet man die absoluten Zahlen der Unfälle über die Leistungsgewichte (Abbildung 6-1) und Charakteristiken der Unfallstelle, so ist zu erkennen, dass sich besonders außerorts in Kurven eine Vielzahl an Unfällen ereignen (entsprechend Konstellation 1 in Abbildung 5-18). Dabei sind außerorts Motorräder mit einem geringen Leistungsgewicht (Masse des Motor- rads zu maximale Leistung in $\mathrm{kg} / \mathrm{kW}$ ) überrepräsentiert (Abbildung 6-2). Darauf folgen Unfälle an Einmündungen.

Die Anteile der jeweiligen Unfallschweren sind für Innerorts- in Abbildung 6-3 und für Außerortsunfälle in Abbildung 6-4 dargestellt. Innerorts wie auch außerorts steigt der Anteil der Schwerverletzten und Getöteten mit sinkendem Leistungsgewicht in den jeweiligen Leistungsgewichtsklassen. Außerorts liegt der Anteil der Leichtverletzten nur noch bei $40 \%$. Bei Fahrzeugen über $10 \mathrm{~kg} / \mathrm{kW}$ bei ca. $60 \%$.

Eine differenziertere Auswertung der Daten des Statistischen Bundesamtes bezüglich technischer Eigenschaften ist aufgrund der geringen Informationstiefe nicht möglich. Es wird jedoch durch die große Datenmenge gezeigt, dass ein Zusammenhang zwischen der Verletzungsschwere und dem Leistungsgewicht eines Motorrades besteht.

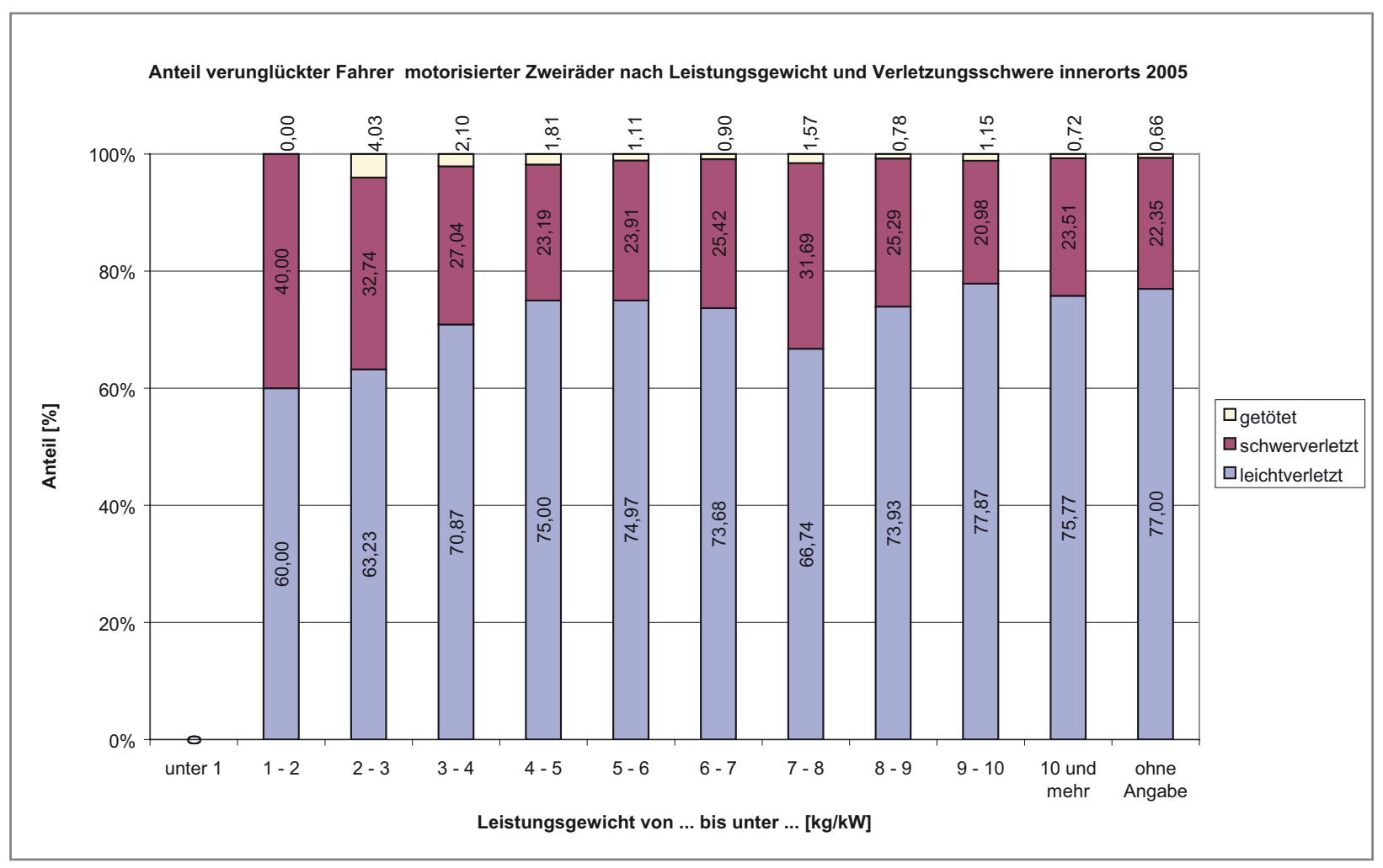

Abbildung 6-3: Anteil der Unfallschwere innerorts nach Leistungsgewicht 


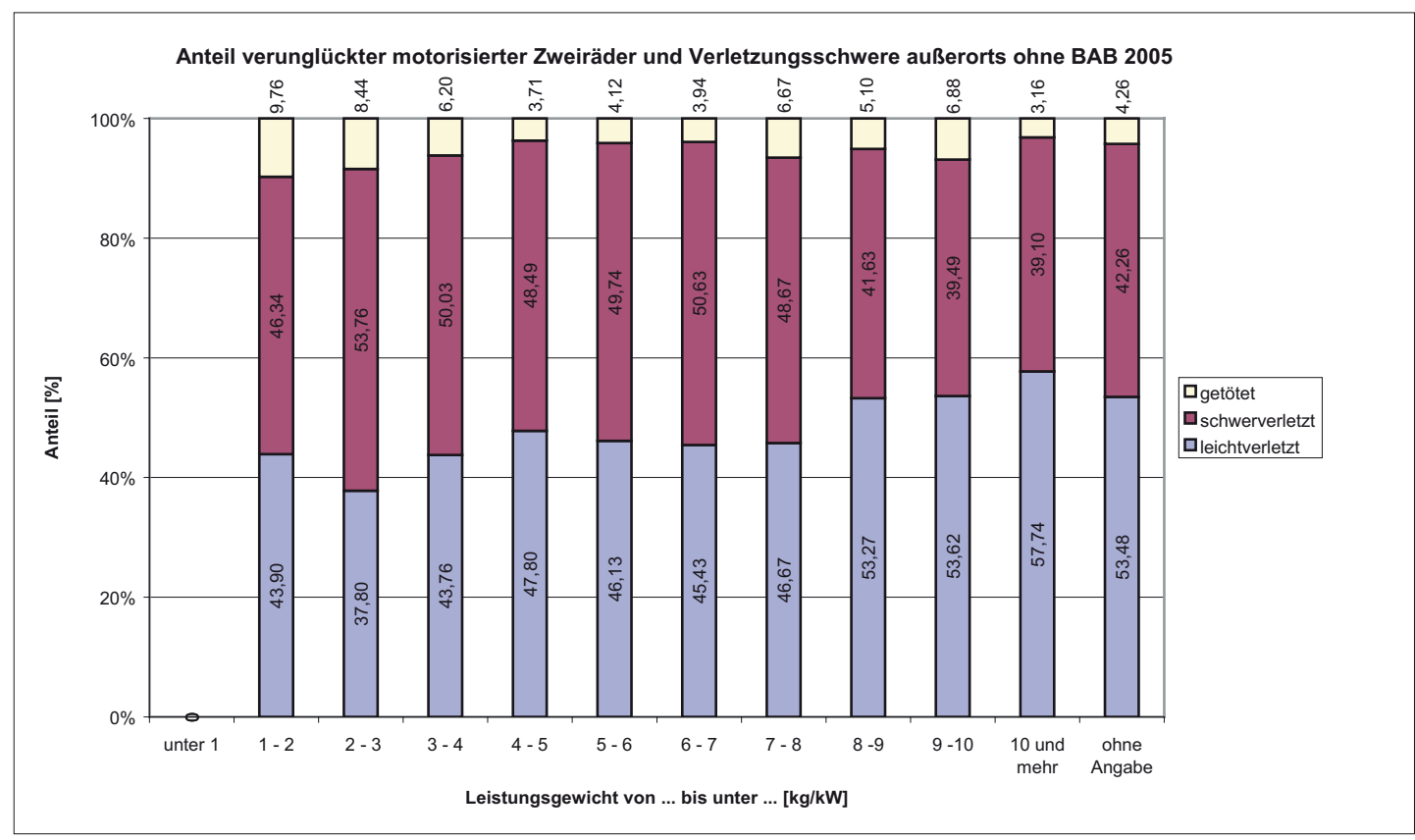

Abbildung 6-4: Anteil der Unfallschwere außerorts nach Leistungsgewicht

\begin{tabular}{|c|c|c|c|c|c|c|c|c|}
\hline Nr. & Bezeichnung & $\begin{array}{l}\text { Unfall- } \\
\text { zahl TU } \\
\text { Dresden }\end{array}$ & $\begin{array}{c}\text { Unfall- } \\
\text { zahl TU } \\
\text { Berlin }\end{array}$ & $\begin{array}{l}\text { Unfall- } \\
\text { art }\end{array}$ & $\begin{array}{c}U \\
(S P) / U \\
(P+S) \\
\text { TUD }\end{array}$ & $\begin{array}{c}\mathrm{U} \\
(\mathrm{SP}) / \mathrm{U} \\
(\mathrm{P}+\mathrm{S}) \\
\text { TUB }\end{array}$ & $\begin{array}{c}\mathrm{U}(\mathrm{P}) / \mathrm{U} \\
(\mathrm{P}+\mathrm{S}) \\
\text { TUD }\end{array}$ & $\begin{array}{c}\mathrm{U}(\mathrm{P}) / \mathrm{U} \\
(\mathrm{P}+\mathrm{S}) \\
\text { TUB }\end{array}$ \\
\hline 1 & $\begin{array}{l}\text { Alleinunfall außerorts mit Abkommen } \\
\text { nach rechts }\end{array}$ & 29 & 21 & 8 & 0,68 & 0,48 & 1,00 & 0,52 \\
\hline 2 & EK-Unfall außerorts an Einmündungen & 9 & 80 & 5 & 0,55 & 0,84 & 0,89 & 0,99 \\
\hline 3 & $\begin{array}{l}\text { Alleinunfall innerorts mit Abkommen } \\
\text { nach rechts }\end{array}$ & 8 & 8 & 8 & 0,63 & 0,63 & 1,00 & 1,00 \\
\hline $4_{-1}$ & EK-Unfall innerorts an Einmündungen & 34 & 75 & 5 & 0,26 & 0,73 & 0,74 & 0,99 \\
\hline $4-2$ & EK-Unfall innerorts an Kreuzungen & 27 & 60 & 5 & 0,22 & 0,78 & 0,70 & 0,95 \\
\hline 5 & $\begin{array}{l}\text { AB-Unfall innerorts an Einmündungen, } \\
\text { Unfallart } 3\end{array}$ & 9 & 57 & 3 & 0,11 & 0,75 & 0,78 & 0,96 \\
\hline 6 & $\begin{array}{l}\text { AB-Unfall innerorts an Kreuzungen, } \\
\text { Unfallart } 4\end{array}$ & 21 & 39 & $4+5$ & 0,24 & 0,88 & 0,76 & 1,00 \\
\hline 7 & $\begin{array}{l}\text { Auffahrunfall innerorts an } \\
\text { Einmündungen/Kreuzungen/Gefälle }\end{array}$ & 36 & 16 & 2 & 0,07 & 0,69 & 0,41 & 0,94 \\
\hline 8 & $\begin{array}{l}\text { Unfall innerorts an Einmündungen/ } \\
\text { Kreuzungen mit Kfz in gleicher Richtung }\end{array}$ & 35 & 11 & 3 & 0,06 & 0,45 & 0,26 & 0,91 \\
\hline 9 & $\begin{array}{l}\text { Fahrunfall innerorts anderer Art } \\
\text { ohne Charakteristik }\end{array}$ & 12 & 0 & 0 & 0,42 & $x$ & 0,92 & $x$ \\
\hline 10 & $\begin{array}{l}\text { Auffahrunfall innerorts ohne Charakte- } \\
\text { ristik }\end{array}$ & 24 & 0 & 2 & 0,04 & $x$ & 0,33 & $x$ \\
\hline
\end{tabular}




\subsection{Auswertung der Daten des GDV}

Die auffälligen Unfallkonstellationen sind in den Daten des GDV mit folgender Häufigkeit vertreten (Tabelle $6-1)$.

Für eine statistisch begründete Auswertung werden nur Konstellationen herangezogen, bei denen mehr als 30 Fälle auftreten. Durch die starke Spezialisierung dieser Konstellationen durch Örtlichkeit, Unfalltyp und Unfallart sind die Häufigkeiten in den Datensätzen deutlich herabgesetzt. Die Einträge in orange geben neben der Anzahl der Datensätze in den GDV- Daten, sowohl den Anteil der Schwerverletzten und Getöteten an allen Unfällen $(U(S P) / U(P+S))$ als auch den Anteil an Personenschäden bei allen Unfällen $(U(P) / U(P+S))$ wieder. Für die detaillierte Auswertung werden die Unfallkonstellationen 2, 4, 5, 6 und 7 näher betrachtet.

\subsubsection{Verletzungsschwereverteilung}

Die Verteilung der MAIS- Werte² (Abbildung 6-5) zeigt, dass es bei Vorfahrtunfällen außerhalb geschlossener Ortschaften an Einmündungen vermehrt zu tödlichen Unfällen und sehr schweren Verletzungen kommt. Der Unfallverursacher dabei ist zu über 90 \% nicht der Motorradfahrer selbst.

Vergleicht man die MAIS Einteilung mit der Verteilung der Verletzungsschwere entsprechend den Kategorien der Amtlichen Statistik, so ist eine weniger genaue Abbildung des körperlichen Schadens zu erkennen (Abbildung 6-6).

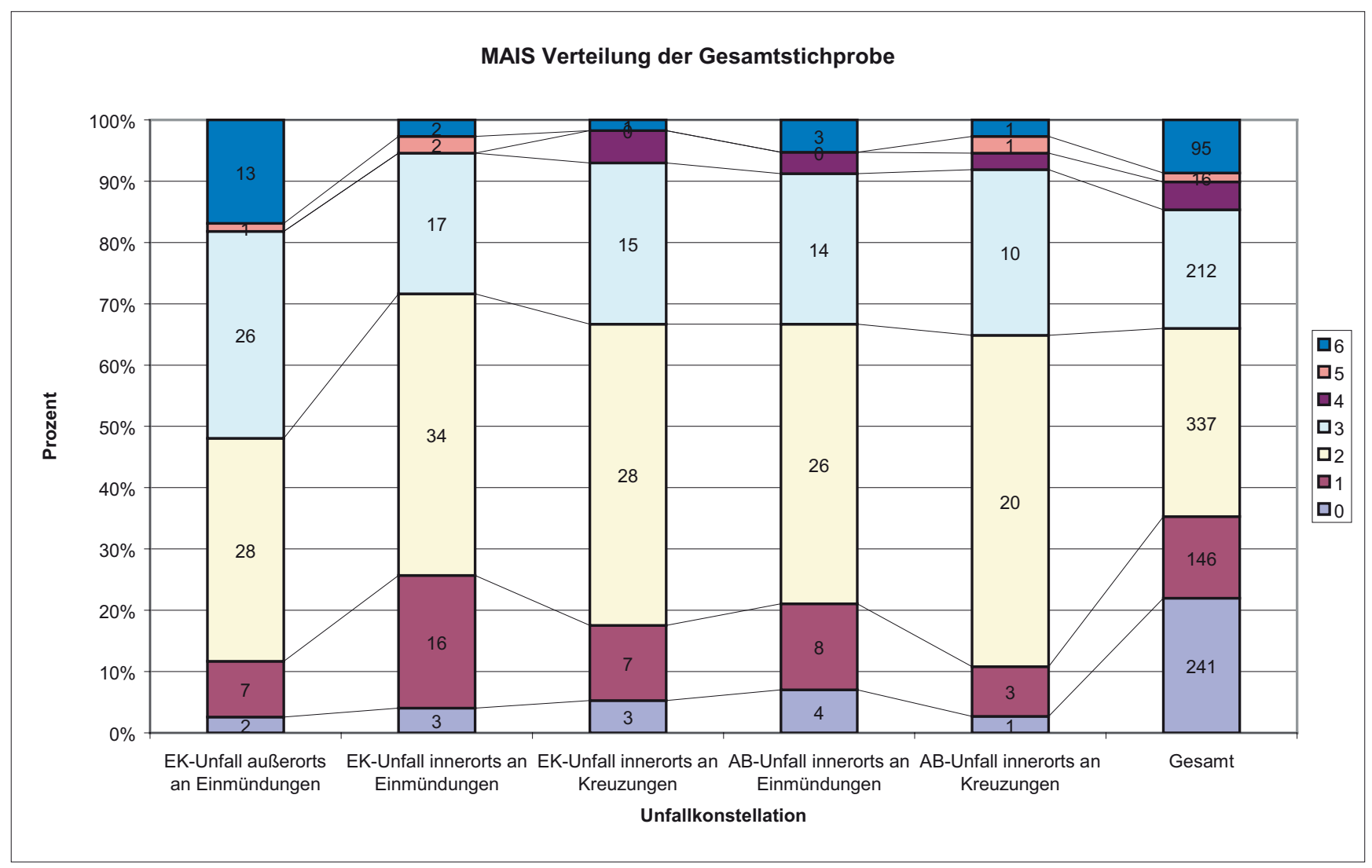

Abbildung 6-5: MAIS Verteilung über die gewählten Unfallkonstellation

2 Maximaler Abbreviated Injury Scale Wert: International anerkannte Ordinalskalierung der Verletzungsschwere für sieben Körperregionen (Kopf, Hals, Thorax, Abdomen, Wirbelsäule, Extremitäten, Körperoberfläche) von 0 bis 6 . Wichtigstes Kriterium ist die von der Verletzungsschwere ausgehende Lebensbedrohung. Der Grad der Lebensbedrohung ist nichtlinearprogressiv und reicht von AIS $0=$ unverletzt (Letalitätsrate $0,00 \%$ ) über AIS $3=$ schwer, nicht lebensgefährlich verletzt (Letalitätsrate $2,91 \%$ ) bis AIS $6=$ maximal verletzt (Letalitätsrate 100\%). (Appel, 2002) 


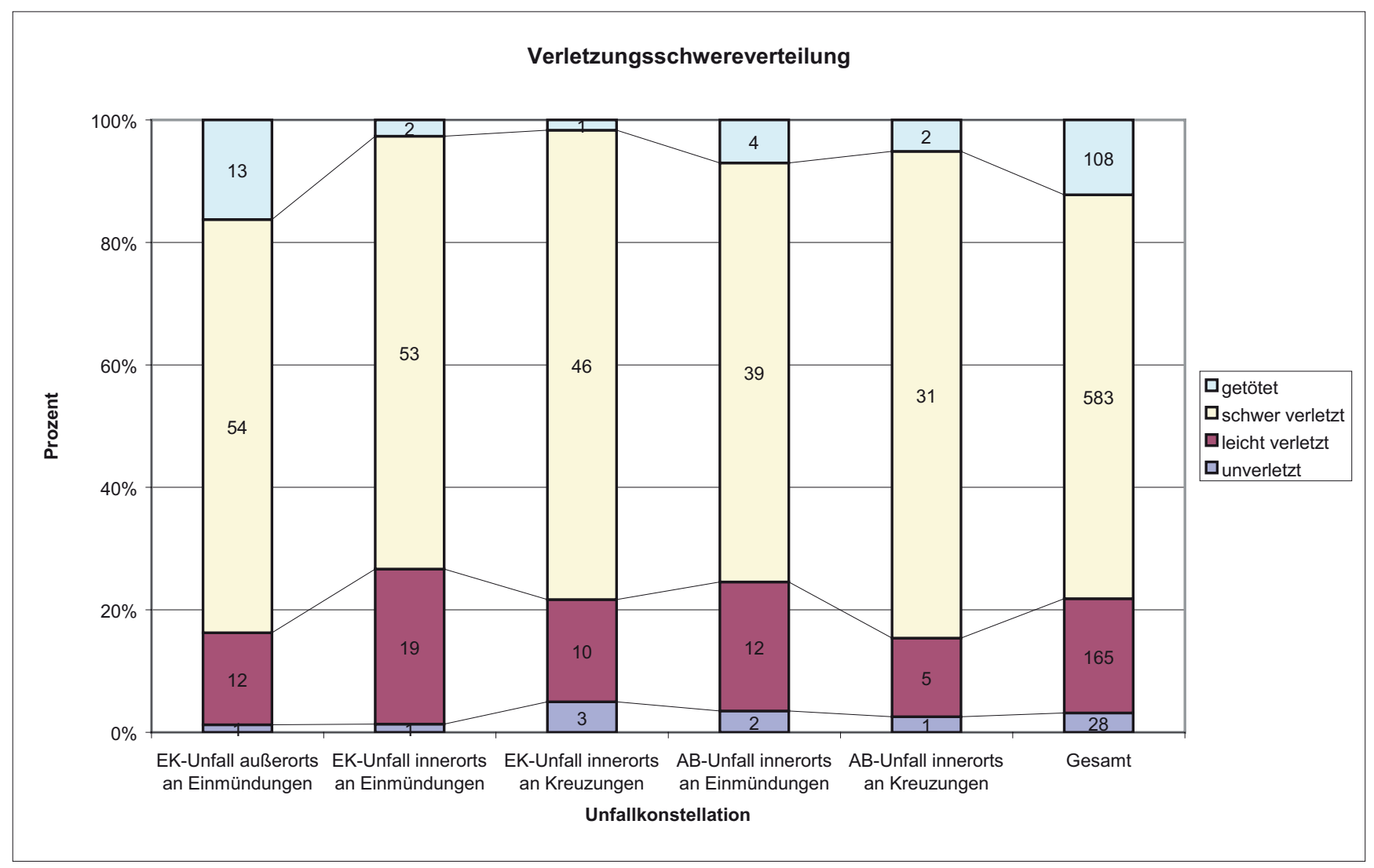

Abbildung 6-6: Verteilung der Verletzungsschwere

\subsubsection{Hauptanstoß}

Abbildung 6-7 zeigt die Verteilung der Hauptanstöße bei unverschuldeten Unfällen des Motorradfahrers. Die selbstverschuldeten Unfälle mit mehr als einem Beteiligten sind nach der vorliegenden Datenauswahl für diese Unfallkonstellationen so gering, dass sie für eine bessere Übersicht nicht dargestellt werden müssen. Zu erkennen ist, dass zu ca. 80 \% der Motorradfahrer an seinem Vorfahrtsrecht gehindert wird, indem der Unfallgegner in seine Bahn fährt und ihn an der rechten Seite trifft oder der Motorradfahrer frontal in dessen linke Seite fährt. Die Verletzungsschwere liegt bei einem frontalen Auftreffen des Motorradfahrers auf den Unfallgegner im Schnitt um einen MAIS- Wert höher, als wenn er selbst an der rechten Seite getroffen wird. Die Verletzungsschweren außerorts liegen wiederum jeweils einen MAIS-Wert über den Werten innerorts. Die gefährlichste Begegnung in diesen Unfallkonstellationen ist somit der Vorfahrtunfall an Einmündungen außerorts, bei der der Motorradfahrer unschuldig in die Seite eines anderen Verkehrsteilnehmers fährt. Dabei sind im Datensatz 12 Todesfälle enthalten, die zusammen $17 \%$ der Frontalzusammenstöße mit Todesfolge ausmachen.

Der Median der MAIS Bewertung einer Verletzung liegt bei Kollision höher als bei Unfällen ohne Kollision (Abbildung 6-8), wenn sich der Unfall außerhalb geschlossener Ortschaften ereignet. So liegt die Verletzungsschwere außerorts mit Kollision um über einen MAIS-Wert höher als bei Unfällen innerorts. 


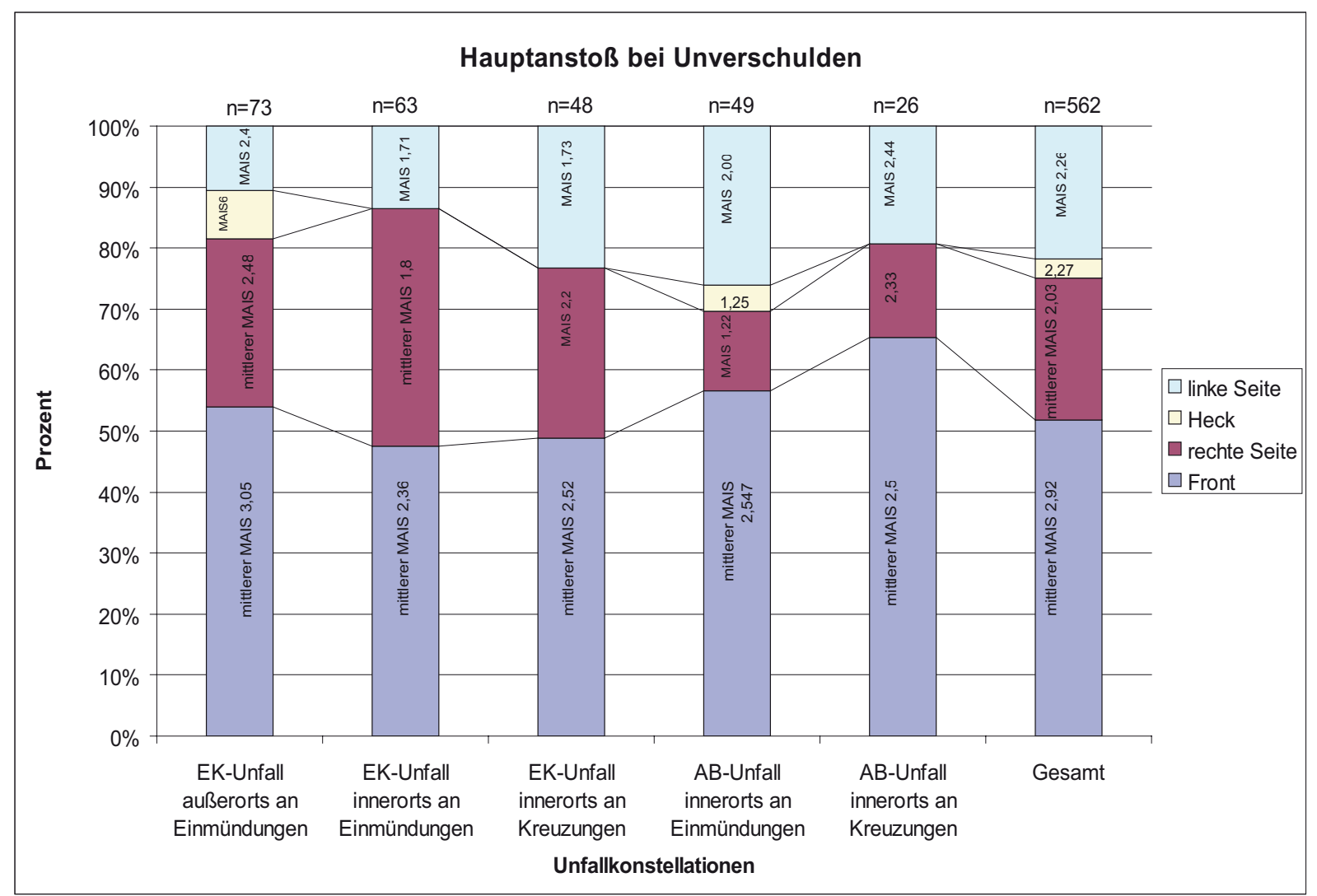

Abbildung 6-7: Hauptanstoß bei Unverschulden

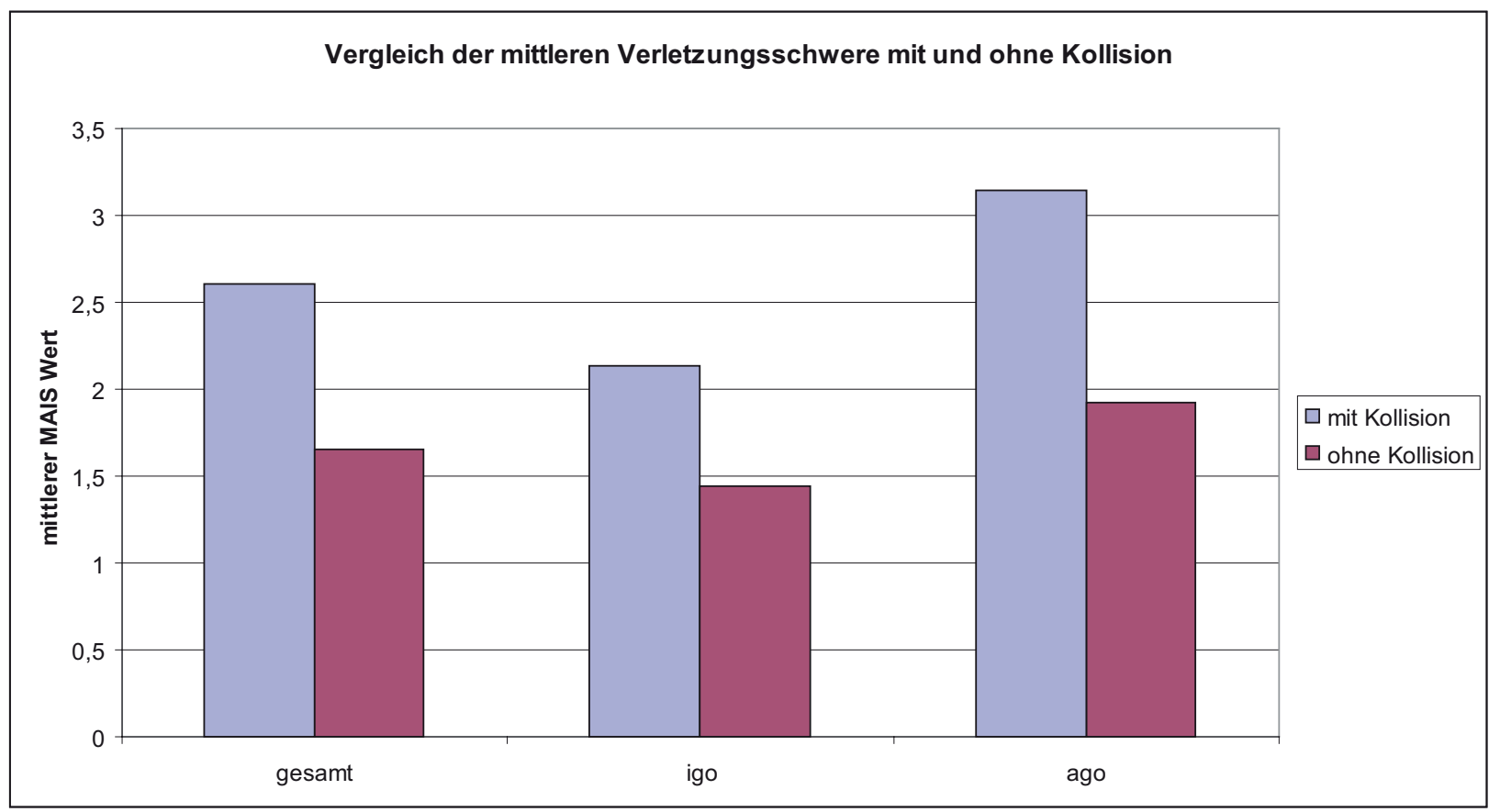

Abbildung 6-8: MAIS Vergleich mit und ohne Kollision 


\subsubsection{Alkohol und überhöhte Geschwindigkeit}

So genannte Diskounfälle sind bei Motorradfahrern selten anzutreffen. Einige Fälle zu Alkohol am Steuer sind jedoch in den Daten zu finden. Insgesamt sind in den gewählten Unfallkonstellationen 9 Unfälle enthalten, die sich unter dem Einfluss von Alkohol ereigneten. Eine statistische Aussagekraft haben sie jedoch nicht und werden daher im weiteren nicht mit einbezogen.

Betrachtet man die Konstellationen innerorts, so gibt es auch eine Erhöhung der Verletzungsschwere, wenn es sich um einen Unfall mit hoher Geschwindigkeit handelt, jedoch in einem geringeren Wert. Auch hier liegt die Hauptverursachung durch den Motorradfahrer bei maximal $30 \%$.

\subsubsection{Motorradtyp}

In der Datenbasis erscheinen die Motorradtypen unterschiedlich auffällig in den Unfallkonstellationen. Abbildung 6-10 zeigt den Anteil der Motorradtypen in den betrachteten Unfallkonstellationen. Zur erkennen ist, das Roller innerorts bei Einbiege- und Kreuzungsunfällen an Kreuzungen überrepräsentiert sind und Enduros an Einmündungen bei Abbiegeunfällen. Naked Bikes sind überdurchschnittlich oft in Einbieg- und Kreuzungsunfälle außerhalb geschlossener Ortschaften involviert.
Tourenmodelle sind im Allgemeinen weniger als andere Motorräder in Unfälle involviert. Betrachtet man jedoch die Unfallschwere, die bei den Unfällen auftreten (Abbildung 6-11), so zeigt sich, dass bei kumulierten MAIS-Werten eine stärkere Belastung von Sportmaschinenfahrern gegeben ist. Ihre Verletzungsschwere liegt im Schnitt etwa 0,5 bis 1,0 MAIS-Werte oberhalb der anderen Motorradtypen.

\subsubsection{Leistungsgewicht}

[Elliot 2003] beschreibt in einer britischen Studie, dass der einflussreichste Faktor auf das Unfallszenario das Leistungsgewicht der betroffenen Motorräder sei. Es vereint mehrere technische Eigenschaften des Motorrades, die das Beschleunigungsvermögen des Fahrzeugs beschreiben. Hubraum und Nenndrehzahl haben direkten Einfluss auf die maximale Leistung eines Verbrennungsmotors [Pucher 2004]. Zudem erlaubt ein leichteres Motorrad bei gleicher Leistung eine höhere Beschleunigung, der Wert des Beschleunigungsvermögens steigt mit sinkender Leistungsgewichtzahl $[\mathrm{kg} / \mathrm{kW}]$. Zur besseren Vergleichbarkeit werden zehn gleichgroße Leistungsgewichtklassen gebildet (Tabelle 6-2). Diese teilen die Datenmenge der ausgewählten Konstellationen in jeweils $10 \%$ große Bereiche.

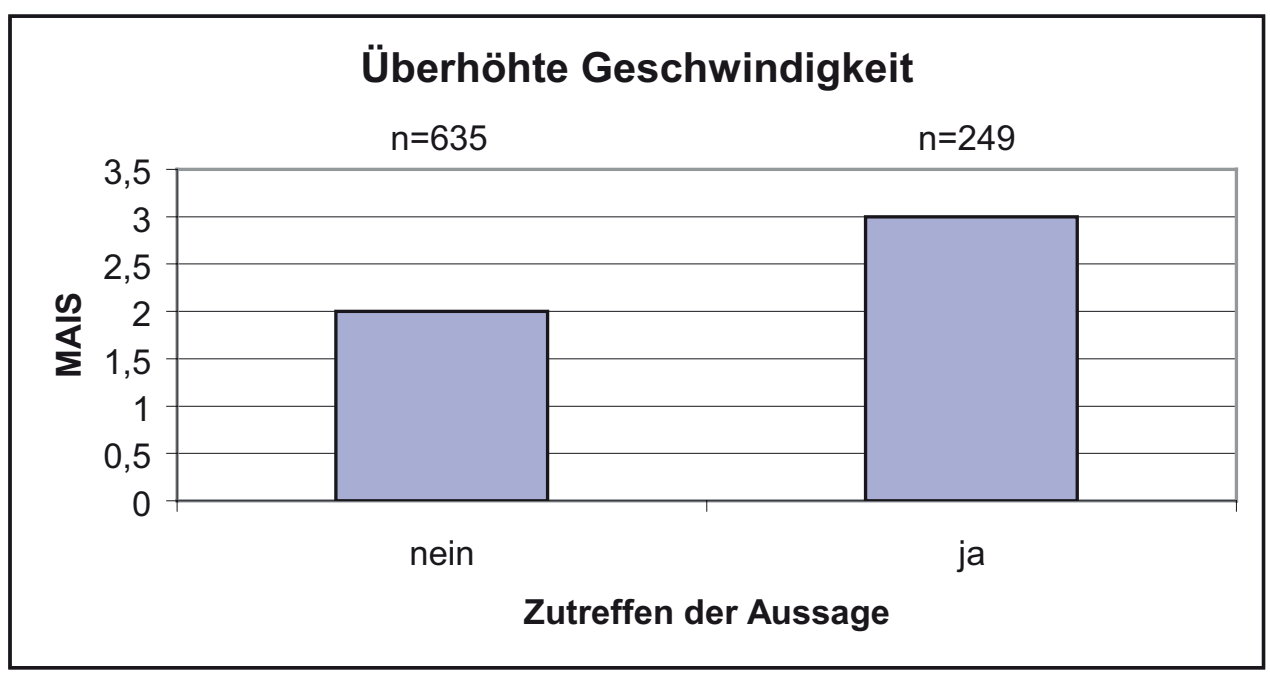

Abbildung 6-9: Alkohol und hohe Geschwindigkeit 
Anteil der Motorräder an Unfällen über die Unfallkonstellation bezogen auf die Häufigkeit der Motorradtypen

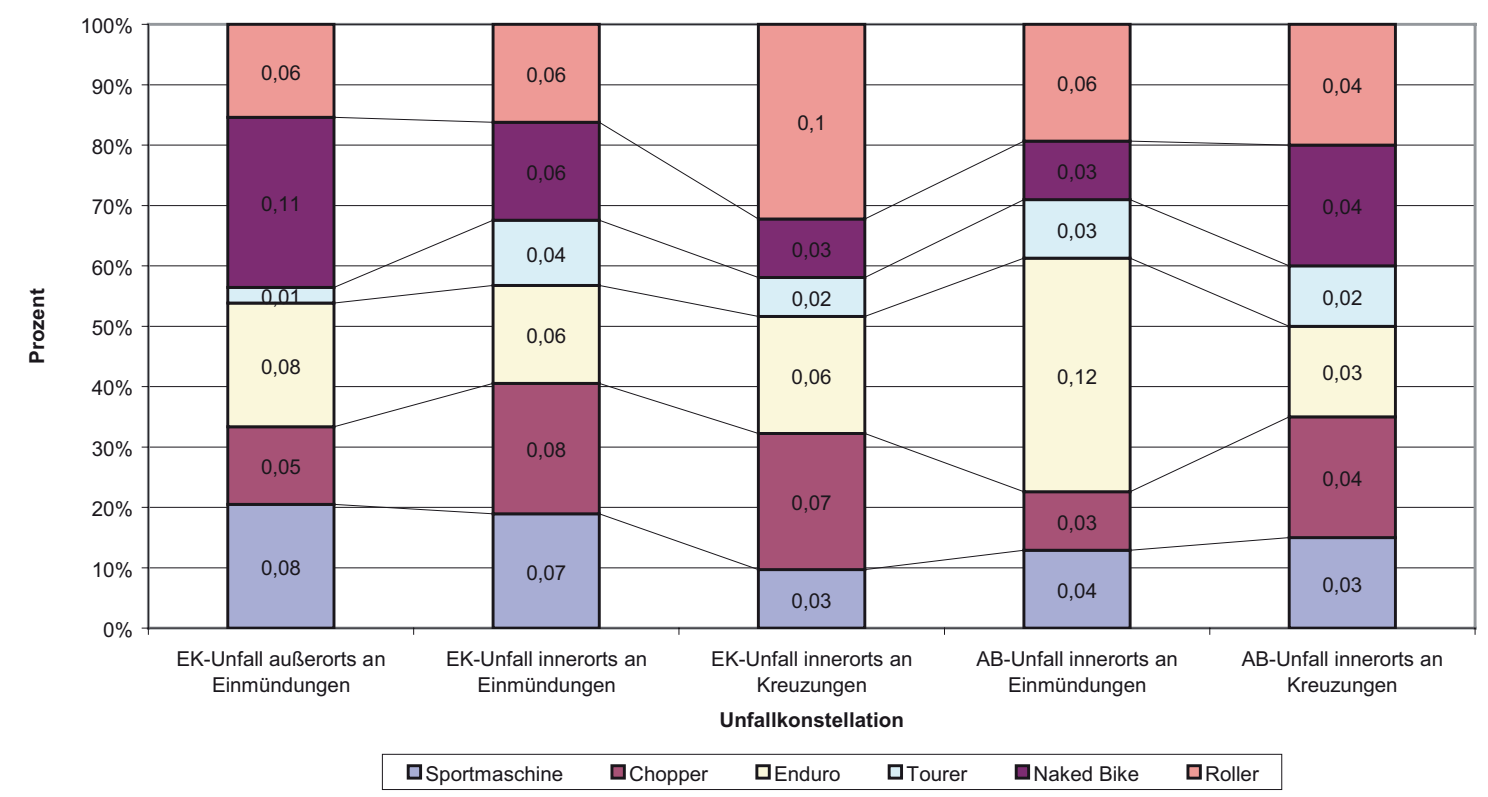

Abbildung 6-10: Auf die Motorradtypenhäufigkeit bezogenes Unfallrisiko

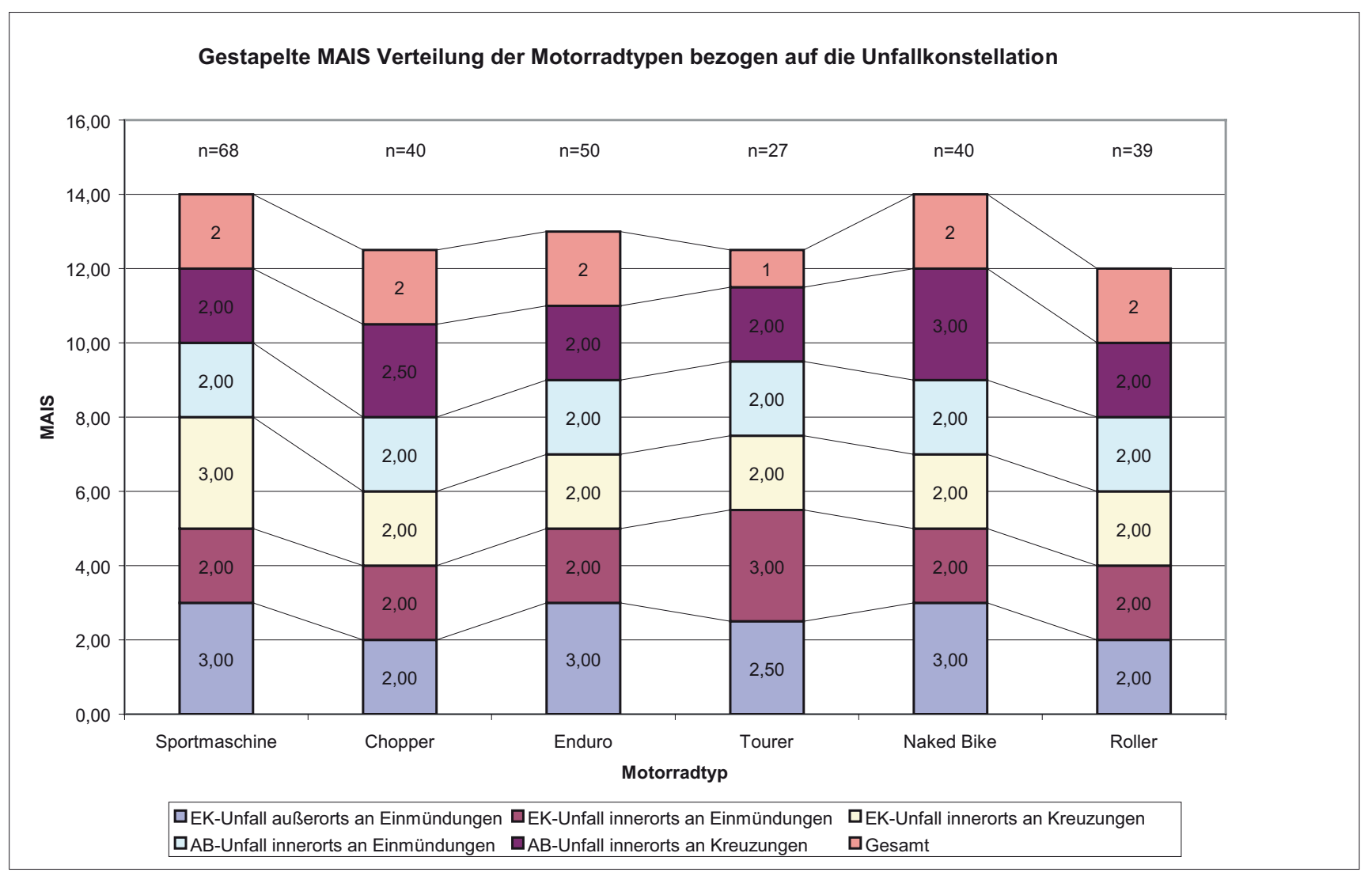

Abbildung 6-11: MAIS der Motorradtypen in den Unfallkonstellationen 


\begin{tabular}{|c|c|}
\hline $\begin{array}{c}\text { Leistungsgewicht- } \\
\text { klasse [1] }\end{array}$ & $\begin{array}{c}\text { Leistungsgewicht } \\
\text { [kg/kW] }\end{array}$ \\
\hline 1 & bis 2,6 \\
\hline 2 & über 2,6 bis 3,1 \\
\hline 3 & über 3,1 bis 3,8 \\
\hline 4 & über 3,8 bis 4,71 \\
\hline 5 & über 4,71 bis 5,9 \\
\hline 6 & über 5,9 bis 7,6 \\
\hline 7 & über 7,6 bis 9,3 \\
\hline 8 & über 9,3 bis 13,4 \\
\hline 9 & über 13,4 bis 15,6 \\
\hline 10 & über 15,6 \\
\hline
\end{tabular}

Tabelle 6-2: Einteilung der Leistungsgewichte in 10 gleich große Klassen

Aufgrund der geringen Fallzahlen ist jedoch in der Verteilung der Leistungsgewichtklassen keine eindeutige Trennschärfe der Verletzungsschwere über den Unfallkonstellationen zu erkennen (Abbildung 6-13). Innerhalb der Klassen gibt es keine Normalverteilung, weswegen über- oder unterrepräsentative Einzelfälle eine starke Verschiebung des Mittelwerts zur Folge haben. Tendenziell lässt die Grafik erkennen, dass geringere Leistungsgewichte ein höheres Verletzungsrisiko bedingen (Abbildung 6-12).

Betrachtet man die gesamte Datenmenge der der Unfallforschung der Versicherungswirtschaft, so ist auch hier kein eindeutiger Zusammenhang zwischen Leistungsgewicht und der Verletzungsschwere des Aufsassen im Fall eines Unfalles zu erkennen (Abbildung 6-14).

Erst die Isolierung derjenigen Fälle im Datensatz, die nach Ortslage und Hauptschuldigkeit des Motorradfahrers unterscheiden, zeigt, dass das Leistungsgewicht Einfluss auf die Verletzungsschwere nimmt (Abbildung 6-15). Fahrer von Motorrädern mit einem hohen Beschleunigungsvermögen haben außerorts bei selbstverschuldeten Unfällen ein erhöhtes Verletzungsrisiko.

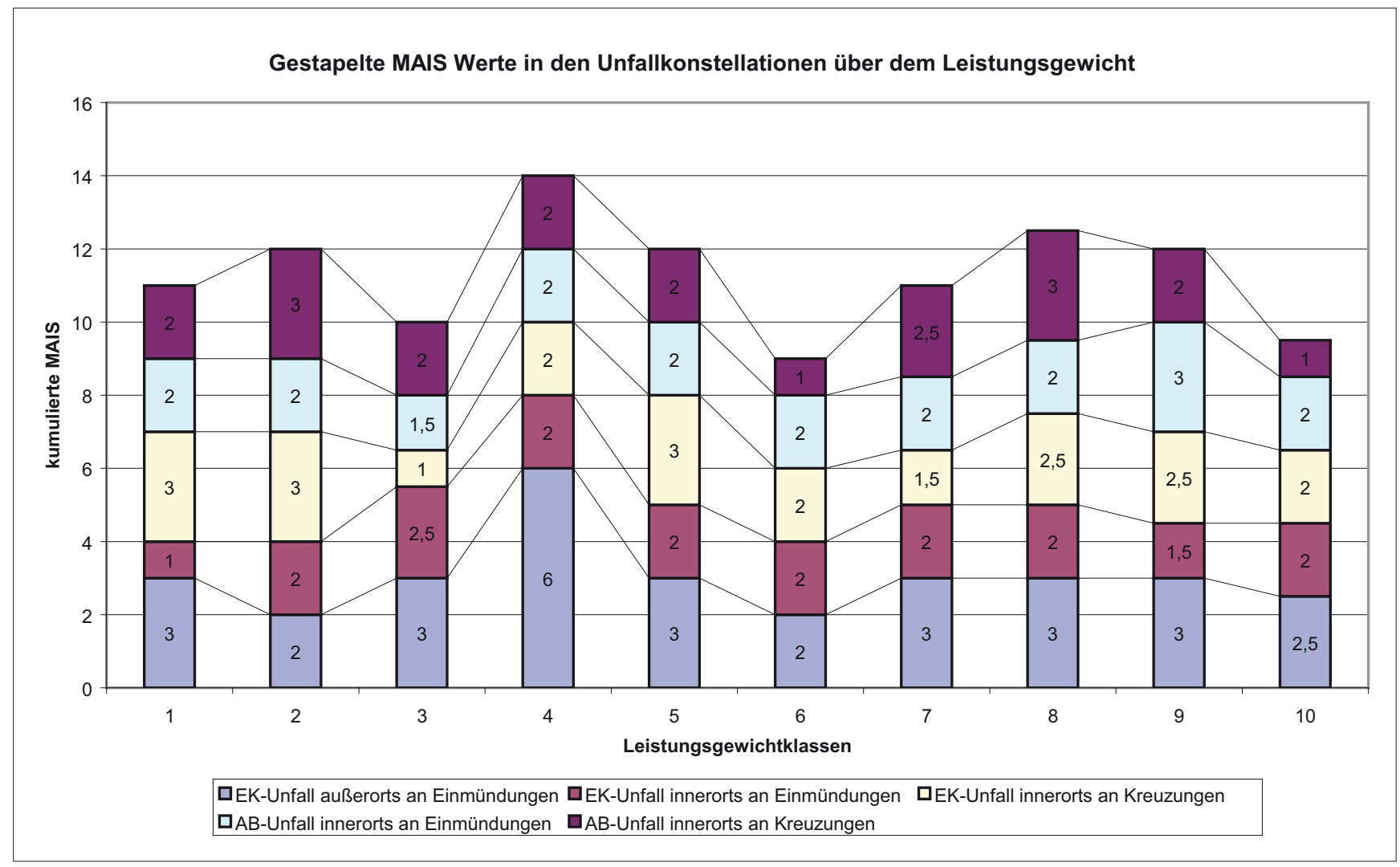

Abbildung 6-12: Mittlere MAIS-Werte der Leistungsgewichtklassen und Unfallkonstellationen 


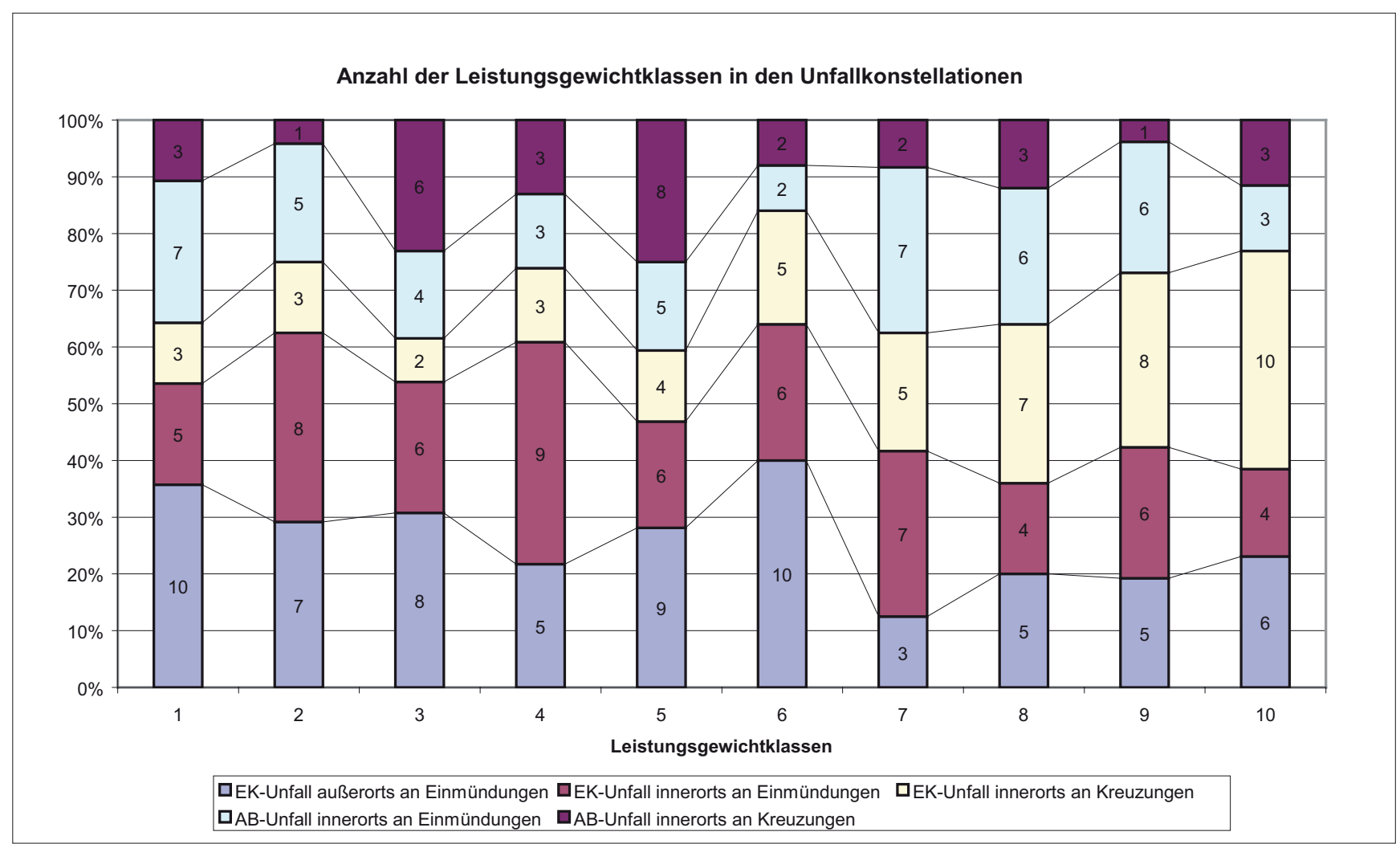

Abbildung 6-13: Anzahl der Leistungsgewichtklassen in den Unfallkonstellationen

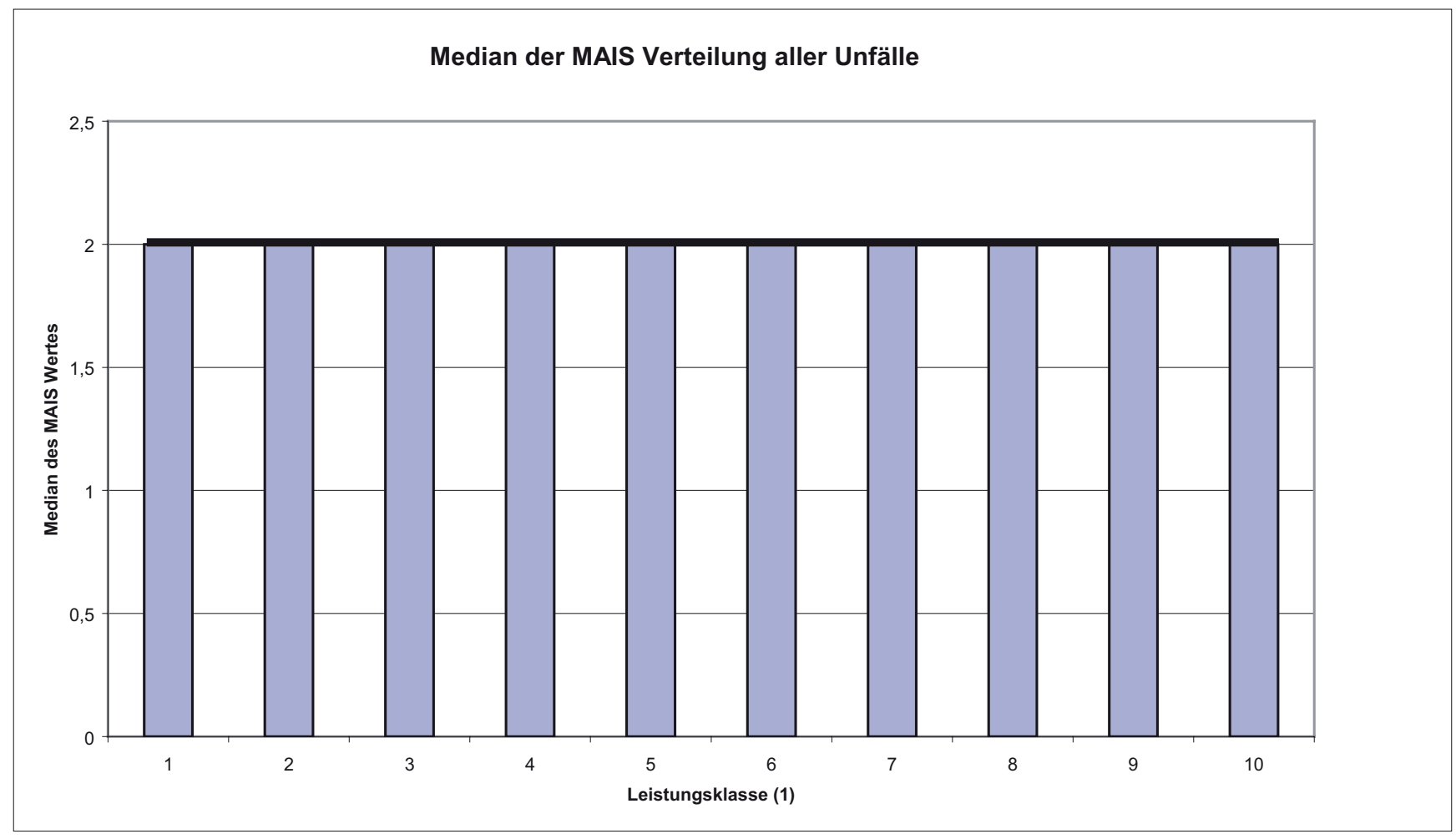

Abbildung 6-14: Mittlere MAIS-Werte der gesamten Leistungsgewichtklassen 


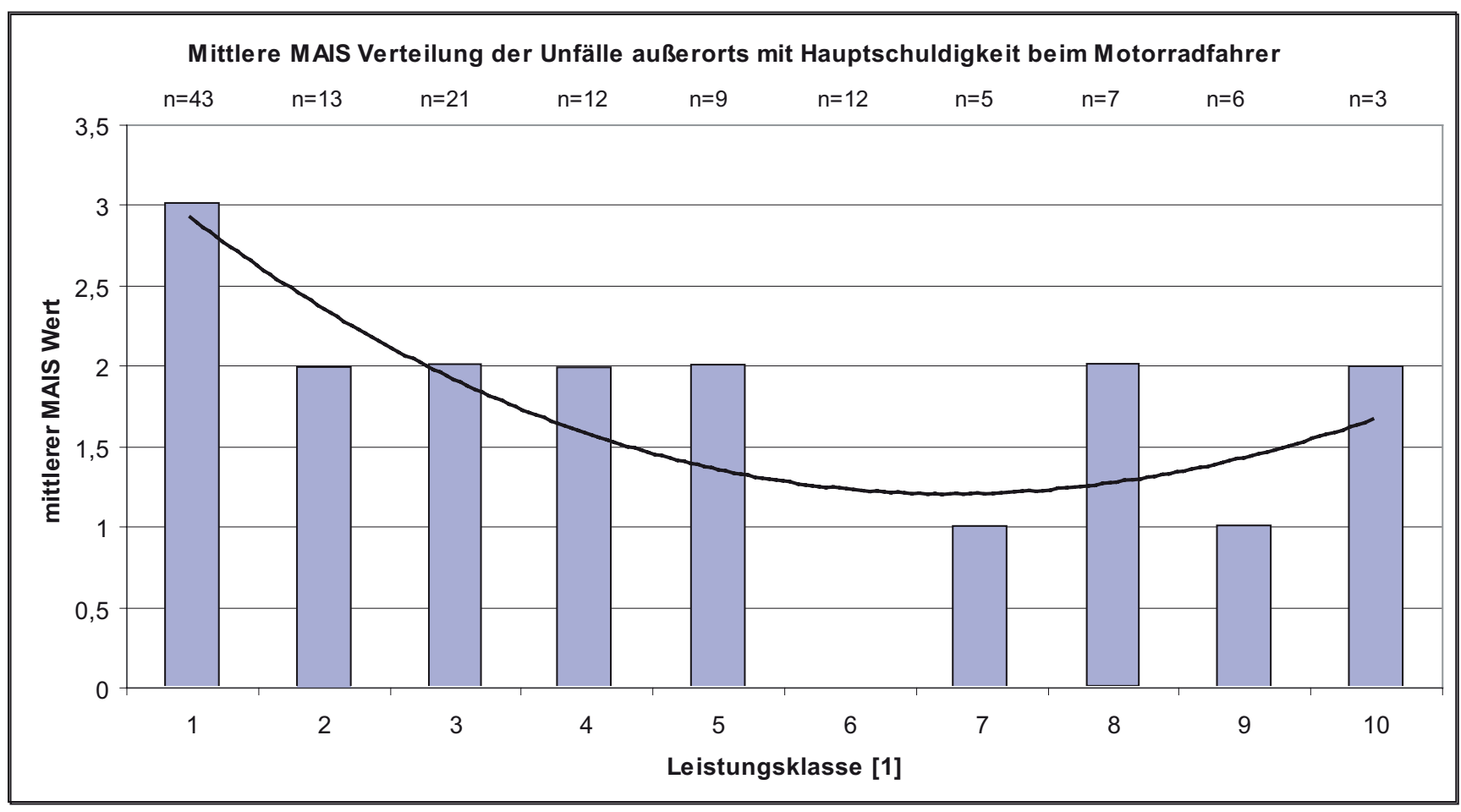

\section{Abbildung 6-15: Verletzungsschwere über Leistungsgewicht ago mit Hauptschuldigkeit PTW}

Erklärt werden kann es dadurch, dass das mögliche Beschleunigungsvermögen vom Motorradfahrer genutzt wird, wenn es zur Verfügung steht und zu risikobereiterem Fahren verleitet. Im Datensatz der Motorradfahrerbefragung ist ein eindeutiger Zusammenhang zwischen Einstellung zur Beschleunigung eines Fahrzeugs, den Verstößen im Straßenverkehr und den Verletzungsschweren zu erkennen (vgl. 6.3.7). Ist der Motorradfahrer nicht der Hauptverursacher einer Kollision, so hat das Leistungsgewicht keinen direkten Einfluss auf den Unfallhergang. Ist er der Hauptunfallverursacher - durch das Beschleunigungsvermögen des Motorrades verleitet - auf einem bestimmten Streckenabschnitt, so hat das Leistungsgewicht direkten Einfluss auf die Schwere des Unfalls. Alleinunfälle des Motorradfahrers sind in diesem Zusammenhang nicht auffällig, da sie großteils ohne jegliche Kollision ablaufen. Der Motorradfahrer wird in der Regel auf die Straße oder neben die Straße in unbefestigtes Gelände geschleudert, was zu weniger schwerwiegenden Verletzungen führt (vgl. 6.2.11). Auch sind Verletzungen der Fahrer von leistungsstarken Motorrädern mit geringem Gewicht durch Unfälle innerorts nicht auffällig höher als die der weniger spurtstarken Einspurfahrzeuge, da durch die im Vergleich zu außerorts geringeren Geschwindigkeiten im Mittel geringere MAISWerte erreicht werden.

Eine Varianzanalyse der Daten zeigt, dass das Leistungsgewicht einen hoch signifikanten Einfluss auf die Verletzungsschwere des Motorradfahrers hat (Tabelle 6-5). Die aufgestellte Hypothese und Alternativhypothese lauten:

$H_{0}$ : Das Leistungsgewicht hat keinen Einfluss auf die Verletzungsschwere

$\mathrm{H}_{1}$ : Das Leistungsgewicht hat einen Einfluss auf die Verletzungsschwere

Das statistische Modell prüft den Zusammenhang zwischen der abhängigen Variablen - der Verletzungsschwere (MAIS) - und mehreren unabhängigen Variablen. Diese sind die Ortslage (Ortslage), die Schuldigkeit des Motorradfahrers (Schuld Motorrad), das Alter des Motorradfahrers (Alter), das Leistungsgewicht des Motorrades (Leistungsgewicht) und deren Interaktionseffekte. 
Für die unabhängigen Variablen Leistungsgewicht und Alter wird eine reduzierte Anzahl an Klassen gebildet (Tabelle 6-3 und 6-4):

\begin{tabular}{|l|l|}
\hline Altersbereich & Klasse \\
\hline unter 18 Jahre & $1(n=10)$ \\
\hline 18 bis 25 Jahre & $2(n=18)$ \\
\hline 26 bis 40 Jahre & $3(n=34)$ \\
\hline über 40 Jahre & $4(n=25)$ \\
\hline
\end{tabular}

Tabelle 6-3: Altersklassen

\begin{tabular}{|l|l|}
\hline Leistungsgewicht $\left[\frac{k g}{k W}\right]$ & Klasse \\
\hline unter 2,75 & $1(n=16)$ \\
\hline 2,75 bis 4,13 & $2(n=31)$ \\
\hline 4,13 bis 9,42 & $3(n=24)$ \\
\hline über 9,42 & $4(n=16)$ \\
\hline
\end{tabular}

Tabelle 6-4: Leistungsklassen
Sie erklären zusammen 42,65 \% der Gesamtvarianz der Verletzungsschwere des Motorradfahrers welche durch das korrigierte Bestimmtheitsmaß $\bar{R}^{2}=0,426$ dargestellt wird. Dabei hat, mit einer Irrtumswahrscheinlichkeit von $\alpha=0,5$, das Leistungsgewicht einen hochsignifikanten Einfluss auf die Verletzungsschwere (Sig.>0,000). Damit kann die Nullhypothese verworfen werden und es gilt die Alternativhypothese $H_{1}$.

Der Einfluss der jeweiligen Faktoren auf die Varianz der abhängigen Variablen wird durch $\eta^{2}$ dargestellt und berechnet sich mit

$$
\eta^{2}=\frac{S S_{\text {Faktor }}}{S S_{\text {total }}}
$$

wobei $S S_{\text {total }}$ die korrigierten Quadratsummen ${ }^{3}$ wiedergeben.

In der Stichprobe hat die unabhängige Variable Leistungsgewicht einen Einfluss von 16 \% auf die Gesamtvarianz der abhängigen Variablen $\left(\eta^{2}=0,16\right)$. Die Unfallart hat denselben Einfluss, kann jedoch zur Reduzierung der Verletzungsschwere nicht beeinflusst werden.

Tests of Between-Subjects Effects

Dependent Variable: MAIS

\begin{tabular}{|c|c|c|c|c|c|c|}
\hline Source & $\begin{array}{c}\text { Type III Sum } \\
\text { of Squares } \\
\text { (SS) }\end{array}$ & df & Mean Square & $\mathrm{F}$ & Sig. & $\eta^{2}$ \\
\hline Corrected Model & $225,612(a)$ & 28 & 8,058 & 3,282 & ,000 & \\
\hline Intercept & 66,382 & 1 & 66,382 & 27,040 & ,000 & \\
\hline Alter & 36,651 & 3 & 12,217 & 4,976 & ,000 & 0,10 \\
\hline Leistungsgewicht & 59,954 & 3 & 19,985 & 8,141 & ,000 & 0,16 \\
\hline Anstoß & 12,841 & 5 & 2,568 & 1,046 & ,400 & 0,03 \\
\hline Unfalltyp & 2,888 & 4 & ,722 & ,294 & ,881 & 0,01 \\
\hline Unfallart & 60,123 & 8 & 7,515 & 3,061 & ,006 & 0,16 \\
\hline Alter * Leistungsgewicht & 16,546 & 5 & 3,309 & 1,348 & ,257 & 0,04 \\
\hline Error & 142,388 & 58 & 2,455 & & & \\
\hline Total & 1151,000 & 87 & & & & \\
\hline Corrected Total & 368,000 & 86 & & & & \\
\hline
\end{tabular}

a R Squared $=, 613$ (Adjusted R Squared $=, 426$ )

\section{Tabelle 6-5: Varianzanalyse der Verletzungsschwere als abhängige Variable}

3 Summe der Quadrate der Abweichungen der Stichprobenwerte vom Mittelwert 
Dependent Variable: MAIS

Multiple Comparisons

Scheffe

\begin{tabular}{|c|c|c|c|c|c|c|}
\hline \multirow[t]{2}{*}{ (I) LG (Binned) } & \multirow[t]{2}{*}{ (J) LG Binned } & Mean Difference (I_J) & Std. Error & Sig. & \multicolumn{2}{|c|}{$95 \%$ Confidence Interval } \\
\hline & & Lower Bound & Upper Bound & Lower Bound & Upper Bound & Lower Bound \\
\hline \multirow[t]{3}{*}{1} & 2 & $2,0766\left(^{*}\right)$ & ,48231 & ,001 & 6879 & 3,4654 \\
\hline & 3 & $1,6250\left(^{*}\right)$ & ,50569 & ,022 & 1689 & 3,0811 \\
\hline & 4 & $2,3750\left(^{*}\right)$ & ,55396 & ,001 & ,7800 & 3,9700 \\
\hline \multirow[t]{3}{*}{2} & 1 & $-2,0766\left(^{*}\right)$ & ,48231 & ,001 & $-3,4654$ &,- 6879 \\
\hline & 3 &,- 4516 & 42601 & ,772 & $-1,6782$ & ,7750 \\
\hline & 4 & ,2984 & ,48231 & ,943 & $-1,0904$ & 1,6871 \\
\hline \multirow[t]{3}{*}{3} & 1 & $-1,6250\left(^{*}\right)$ & ,50569 & ,022 & $-3,0811$ &,- 1689 \\
\hline & 2 & ,4516 & ,42601 & ,772 &,- 7750 & 1,6782 \\
\hline & 4 & ,7500 & ,50569 & ,536 &,- 7061 & 2,2061 \\
\hline \multirow[t]{3}{*}{4} & 1 & $-2,3750\left(^{*}\right)$ & ,55396 & ,001 & $-3,9700$ &,- 7800 \\
\hline & 2 & $-2,984$ & ,48231 & ,943 & $-1,6871$ & 1,0904 \\
\hline & 3 &,- 7500 & ,50569 & ,536 & $-2,2061$ & 0,7061 \\
\hline
\end{tabular}

Based on observed means.

* The mean difference is significant at the ,05 level.

Tabelle 6-6: Post Hoc Test nach Scheffé

Mittels des Post Hoc Test nach Scheffé (Tabelle 6-6) zeigt sich, dass nur Klasse 1 eine signifikant höhere Verletzungsschwere aufweist als die übrigen Klassen. Die Klassen 2 - 4 unterscheiden sich in der Verletzungsschwere nicht. Dies bedeutet, dass Fahrer von Motorrädern mit einem Leistungsgewicht unter $2,75 \mathrm{~kg} / \mathrm{kW}$ höhrere Verletzungsschweren erleiden.

\subsubsection{Unfallbetrachtung nach Wochentagen}

Abbildung 6-16 zeigt die mittleren, wochentäglichen MAIS-Werte bei Motorradunfällen. Insgesamt gibt es keine besonderen Auffälligkeiten. Die Zahlen in den gestapelten Balken geben die Anzahlen der einzelnen Fälle für die jeweiligen Unfallkonstellationen wieder. An Werktagen ereignen sich weniger Unfälle. Die meisten Unfälle geschehen an einem Samstag. Verwunderlich ist dies nicht, da das Motorrad nach der Massenmobilisierung in den 1950er Jahren von einem Fortbewegungsmittel zu einem Freizeitobjekt wechselte. 62 \% der Fahrten mit dem Motorrad in den Unfalldaten des GDV sind reine Freizeit- oder Urlaubsfahrten. Insgesamt liegt hier die Verletzungsschwere auch auf dem höchsten Niveau innerhalb der betrachteten Unfallkonstellationen, doch ist der Unterschied nicht signifikant. Allgemein kann man erkennen, dass die Verletzungsschwere zum Wochenen- de leicht steigt und die Zahl der Unfälle am Samstag nahezu doppelt so hoch ist wie an Wochentagen.

\subsubsection{Verkehrsdichte}

Die Verkehrsdichte, wie sie vom Unfallbeteiligten geschildert wurde, hat innerorts keinen eindeutigen Einfluss auf die Verletzungsschwere der verunfallten Motorradfahrer. Eine leichte Steigerung ist zu erkennen, wenn die Verkehrsdichte steigt (Abbildung 6-17). Dort sind Unfälle mit Kollisionen mit andere Verkehrsteilnehmern wahrscheinlicher, die im allgemeinen zu höheren Verletzungsschweren führen (vgl. 6.2.2).

Auffällig ist, dass außerorts die Verletzungsschwere steigt, wenn die Verkehrsdichte geringer ist. Dieser Zusammenhang ist wiederum durch das Verhalten des Motorradfahrers zu erklären. Die Risikobereitschaft steigt, wenn die Möglichkeit zu einer gefährlichen Fahrweise gegeben ist - ähnlich dem höheren Beschleunigungsvermögen, wenn das Leistungsgewicht geringer ist. Lässt es die Verkehrsdichte zu, so wird mehr (eigenes) Risiko in Kauf genommen. Prinzipiell unterstützt eine geringere Verkehrsdichte das Beschleunigungsvermögen des Motorrades in der jeweiligen Situation. Auf einer leeren Straße kann in einer kürzeren Zeit eine höhere Geschwindigkeit erzielt werden. 


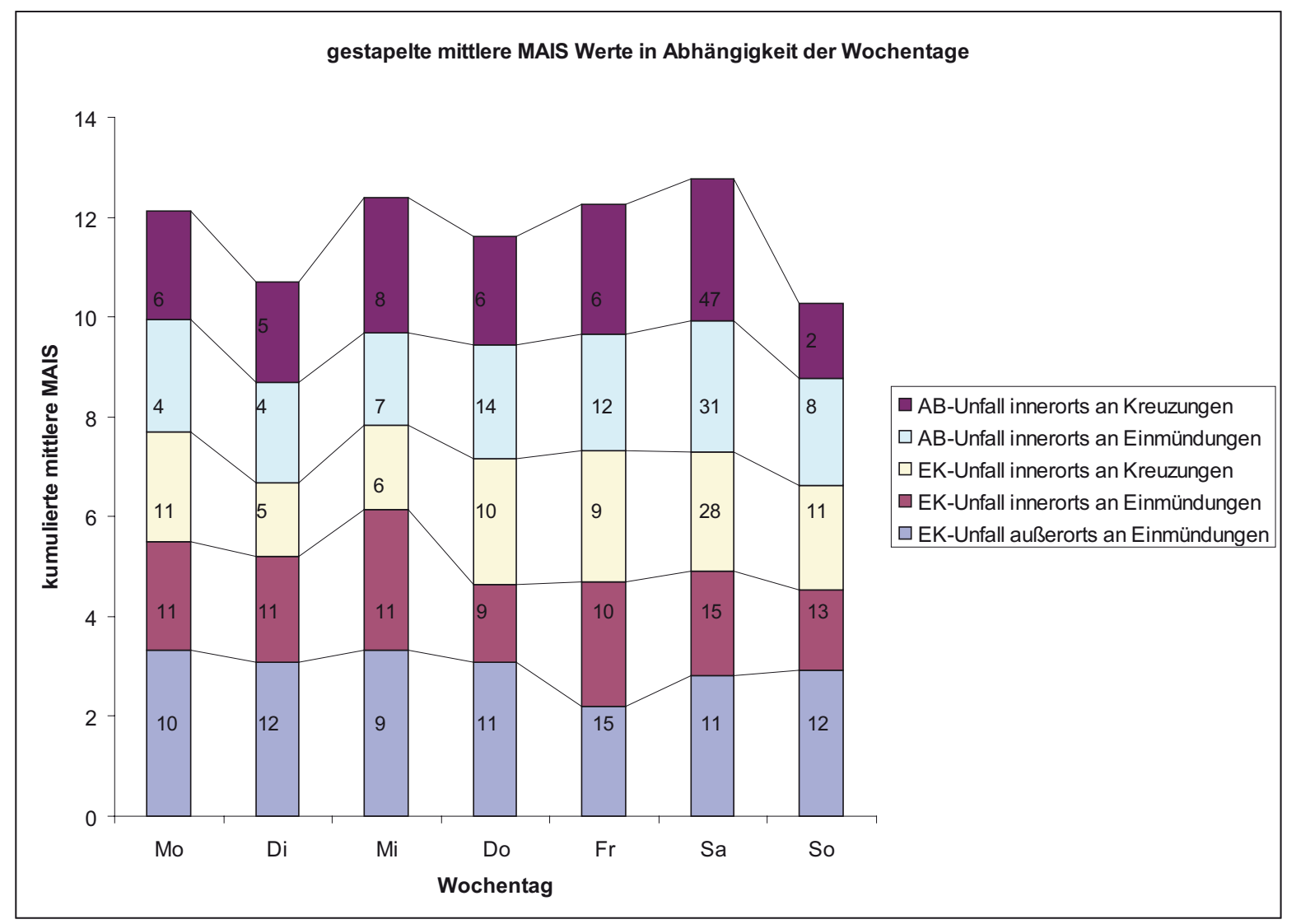

Abbildung 6-16: Mittlere MAIS Verteilung über die Wochentage

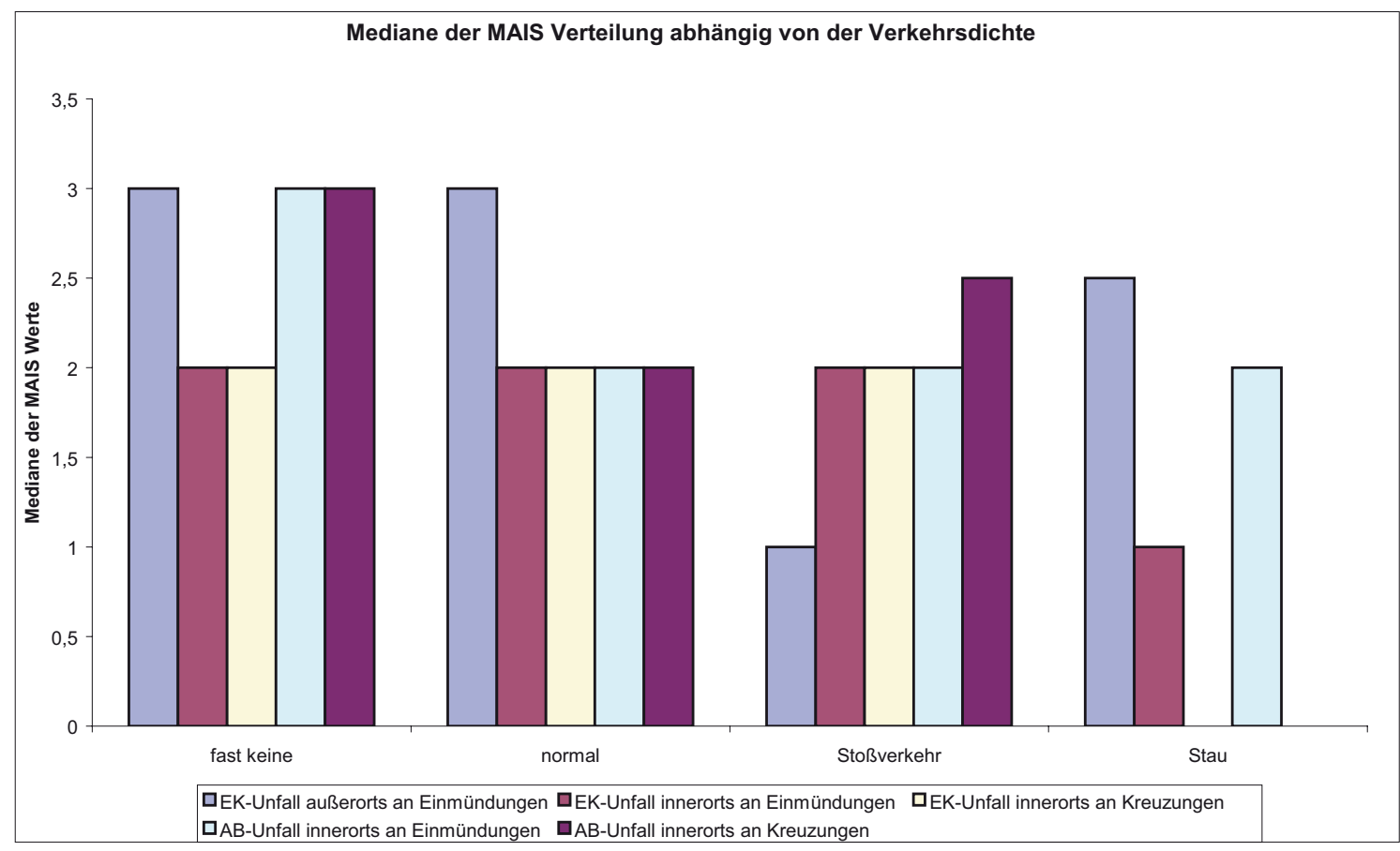

Abbildung 6-17: Mittlere MAIS Verteilung abhängig von der Verkehrsdichte nach Angabe des Beteiligten 


\subsubsection{Reaktion vor einem Unfallereignis}

Schon bei der Führerscheinausbildung in der Fahrschule werden mit dem Neuling auf zwei Rädern Fahrmanöver geübt, die helfen sollen gefährlichen Situationen im StraBenverkehr vorzubeugen. Beherrschung von Gefahrenbremsungen und Ausweichen vor Hindernissen werden als Grundfahrübungen auch in der Prüfung vorausgesetzt.

Bremsen und Ausweichen ist so die wirkungsvollsten Maßnahmen, wenn eine kritische Situation erkannt wird. Zum einen wird die kinetische Energie beim Bremsen

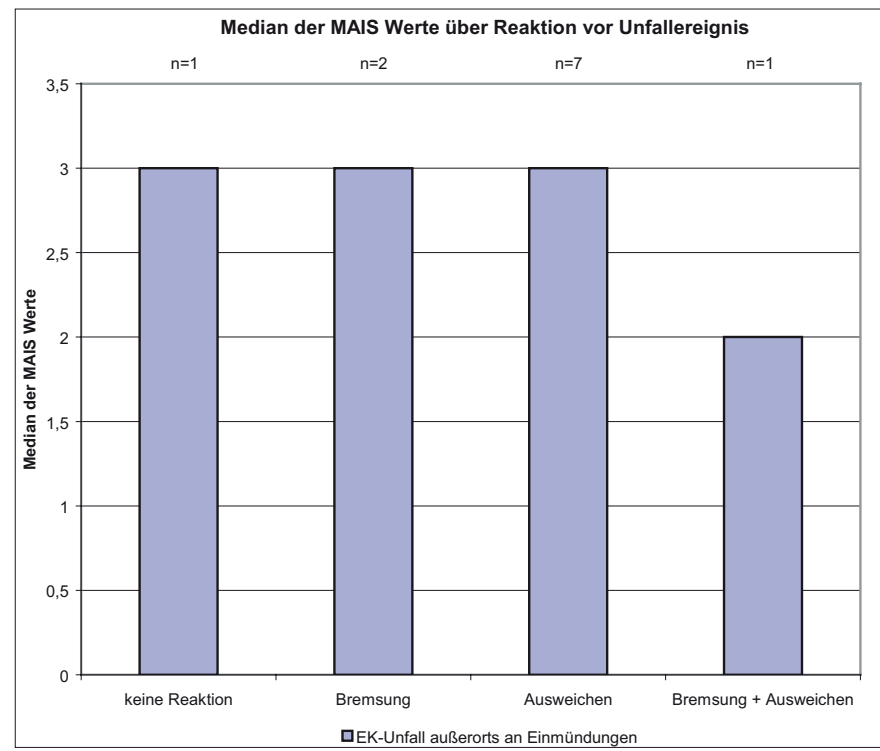

Abbildung 6-18: Verletzungsschwere abhängig der Reaktion vor dem Unfallereignis abgebaut und ein Ausweichen verhindert die Kollision mit einem Unfallgegner. Besonders im Verkehrsraum außerhalb geschlossener Ortschaften hilft dieser Ablauf die mittlere Verletzungsschwere zu senken (Abbildung 6-18). Führt eine Reaktion, wie z. B. falsches Bremsen, zu einem Sturz vor dem Unfallereignis außerhalb geschlossener Ortschaften, so liegt die Verletzungsschwere über anderthalb MAIS-Werte über Unfällen ohne Sturz (Abbildung 6-19). Innerhalb geschlossener Ortschaften gibt es keine signifikanten Unterschiede, da hier die Relativgeschwindigkeiten im Unfallereignis geringer sind.

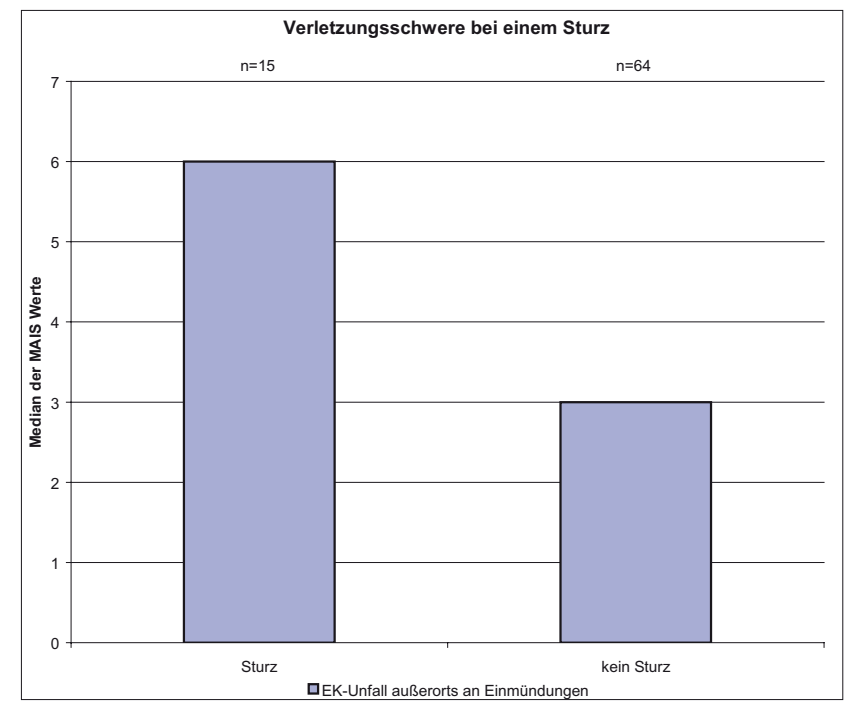

Abbildung 6-19: MAIS-Werte bei Sturz 


\subsubsection{Unfallablauf und Hauptverletzungsregion}

Die Beschreibung des typischen Unfallablaufes und seiner Folgen ist notwendig, um gezielte Maßnahmen zu erarbeiten. Zur Beschreibung des Ablaufes ist der Zustand des Motorrades vor, während und nach dem Unfallereignis dokumentiert worden. Da die gewählten Unfallkonstellationen prinzipiell ähnliche Eigenschaften, wie z. B. Einbiegen und Kreuzen an Einmündungen oder Kreuzungen aufweisen, ist es nicht verwunderlich, dass über alle Unfallkonstellationen ein Ablauftyp am auffälligsten ist. Grundlegend unterschiedlich dazu ist der Alleinunfall.

Abbildung 6-20 zeigt exemplarisch die häufigsten Unfallabläufe am Beispiel des Einbiege- und Kreuzungsunfalls außerhalb geschlossener Ortschaften an Einmündungen. Mit 38 Fällen am häufigsten ist die aufrechte Kollision mit einem Unfallgegner nach einer stabilen Gradeausfahrt. Der mittlere MAIS-Wert liegt hier bei 3.
Wenn der Motorradfahrer vor dem Unfallgegner stürzt, kann er weniger kinetische Energie abbauen und die Verletzungsschwere aufgrund der Kollision ist höher. Geringer sind die Werte, wenn es zu einer Streifkollision kommt oder der Motorradfahrer in einer Kurvenfahrt mit einem anderen Verkehrsteilnehmer kollidiert.

Die am häufigsten betroffenen Körperregionen sind bei den Unfallabläufen mit Kollision zu 30 \% Kopf-, Hals- und Thorax, wenn man nur schwere Verletzungen mit MAIS 3 oder höher betrachtet (Abbildung 6-21).

Alleinunfälle haben einen prinzipiell anderen Unfallablauf mit anderen Verletzungen. Häufigster Unfallablauf ist die stabile Geradeausfahrt oder Kurvenfahrt mit anschließendem Wegrutschen ohne Kollision mit Hindernissen. Besonders betroffen sind die unter Extremitäten mit einer mittleren Verletzungsschwere von MAIS 2,0.

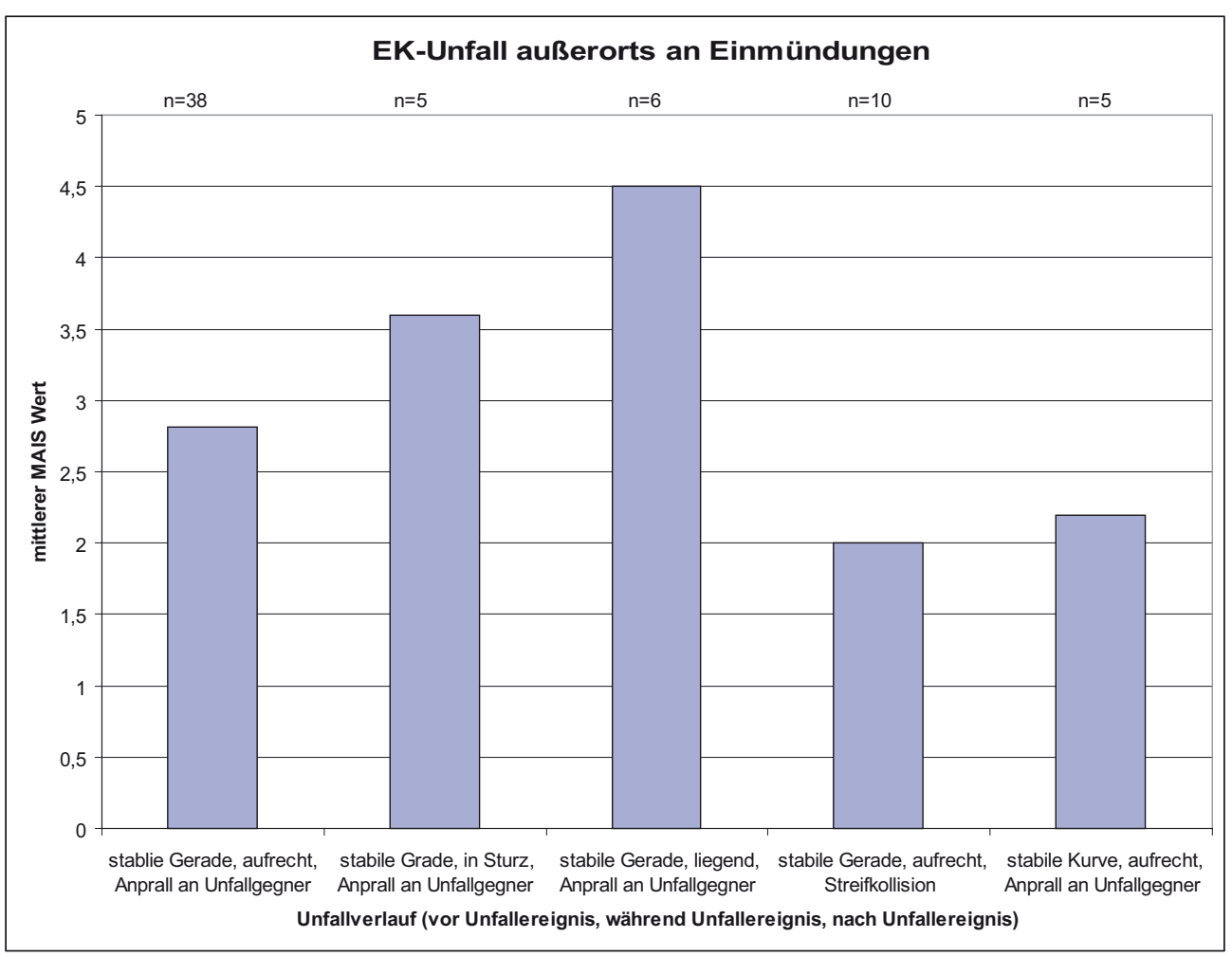

Abbildung 6-20: Unfallhergang exemplarisch am Beispiel EK-Unfall außerorts an Einmündungen 


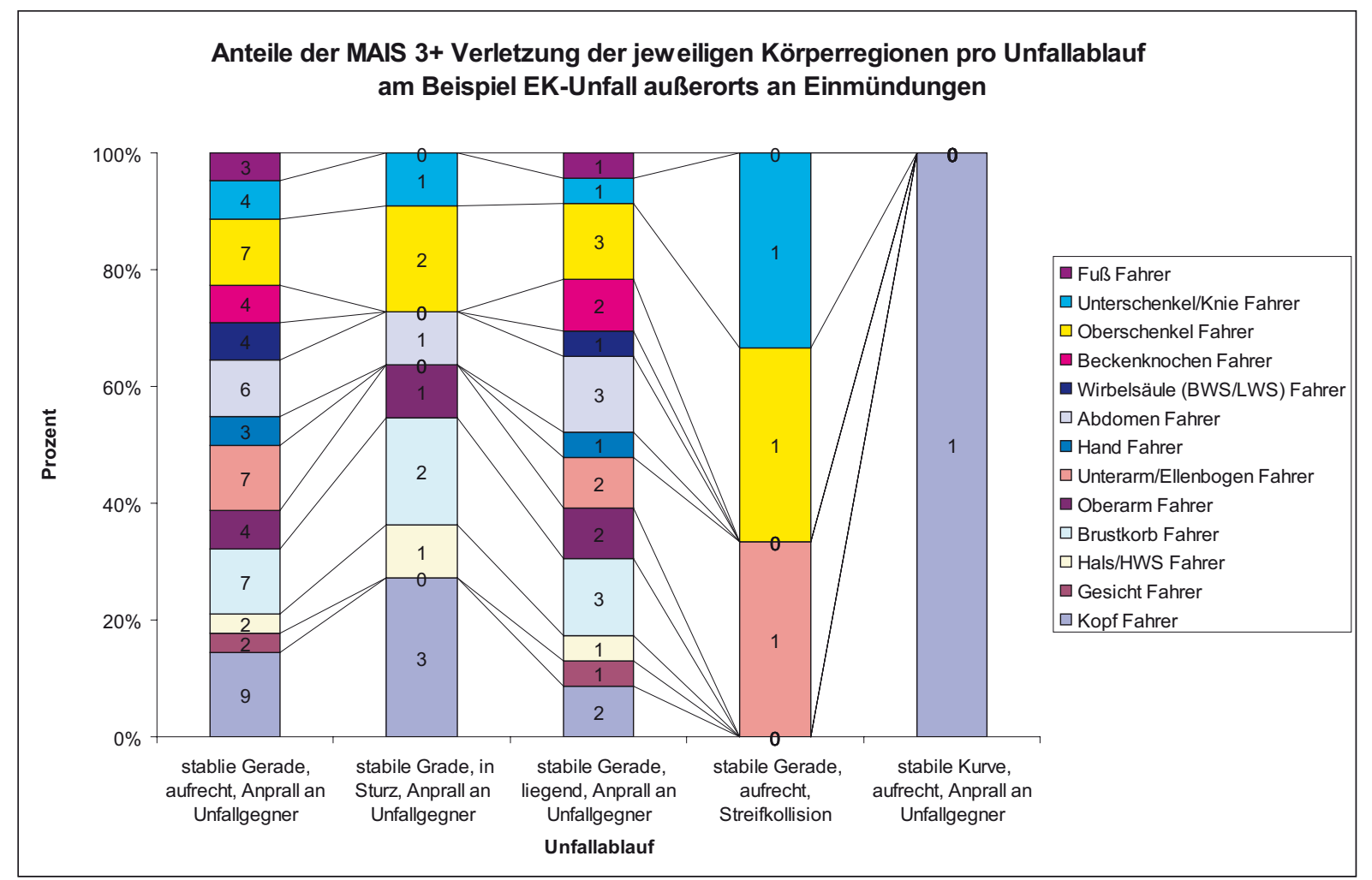

Abbildung 6-21: Anteil der MAIS 3+ Verletzungen der jeweiligen Körperregionen pro Unfallablauf

\subsubsection{0 Überflug im Fall einer Kollision}

Wie in 6.2.2 gezeigt werden konnte, liegt die Verletzungsschwere durchschnittlich um ca. einen MAISWert höher, wenn es bei einem Unfall zu einer Kollision des Fahrers mit dem Unfallgegner kommt. Viele Ansätze zur Verbesserung der passiven Motorradsicherheit versuchen eine Beeinflussung der Flugbahn des Aufsassen. Abbildung 6-22 zeigt die mittlere Verletzungsschwere im Fall eines Überfluges im Vergleich zu Unfällen mit Kollisionen mit dem Unfallgegner. Überfliegt der Motorradfahrer den Gegner, so liegt der Median der MAIS-Werte bei 2 und damit genauso niedrig wie bei Unfällen ohne Kollision. Dies stellt jedoch keine sinnvolle Schwereminderung dar, da die Fahrzeughöhen der Unfallgegner stetig wachsen und die Situation nach einem Überflug nicht beeinflusst werden kann.

\subsubsection{Anteil der Unfallverursacher}

Motorradfahrer sind durch Alleinunfälle außerorts in Verruf gekommen, da diese oft besonders schwerwiegend sind [Maier 2000]. Wie die aktuelle Bundesstatistik [Destatis 2007] zeigt, sterben in der Tat außerorts $44 \%$ der Getöteten bei selbstverschuldeten Unfällen. Bezieht man zudem die Alleinunfälle mit ein, die in der Bundesstatistik als selbstverschuldet geführt werden, so steigt der Anteil der selbst verschuldeten Unfälle mit getöteten Motorradfahrern außerorts auf ein Maximum von $67 \%$.

Betrachtet man die Alleinunfälle in der Datenbank des GDV, so ist zu erkennen, dass ihr Anteil insgesamt bei 10 \% liegt und bei Unfällen mit schwerem Personenschaden oder getöteten Personen bei $12 \%$. Aus der Datenbasis der TU Dresden ergibt sich ein Anteil von $17 \%$ Alleinunfällen in Bezug auf die Unfälle mit PTW-Betei- 


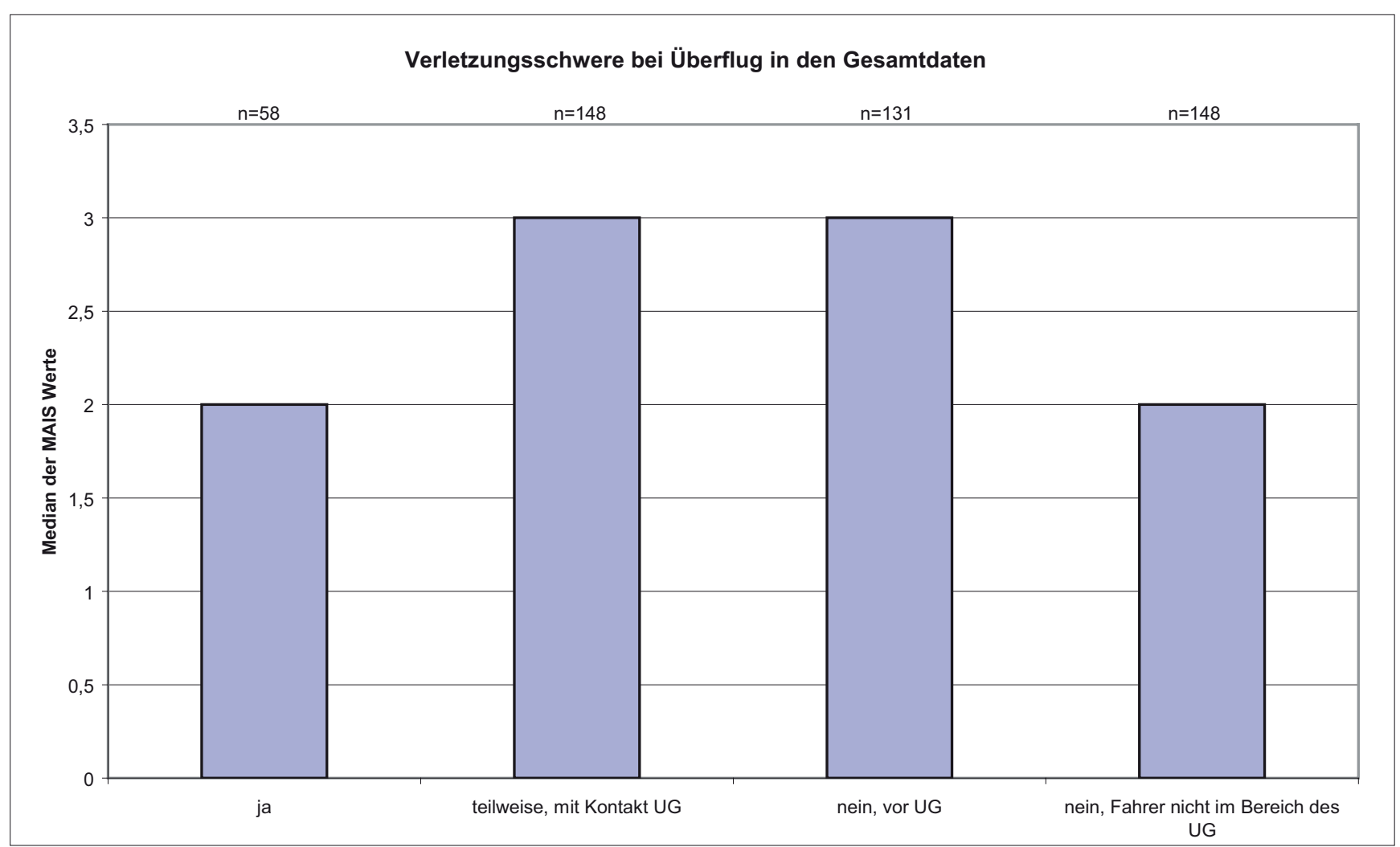

Abbildung 6-22: Verletzungsschwere bei Überflug

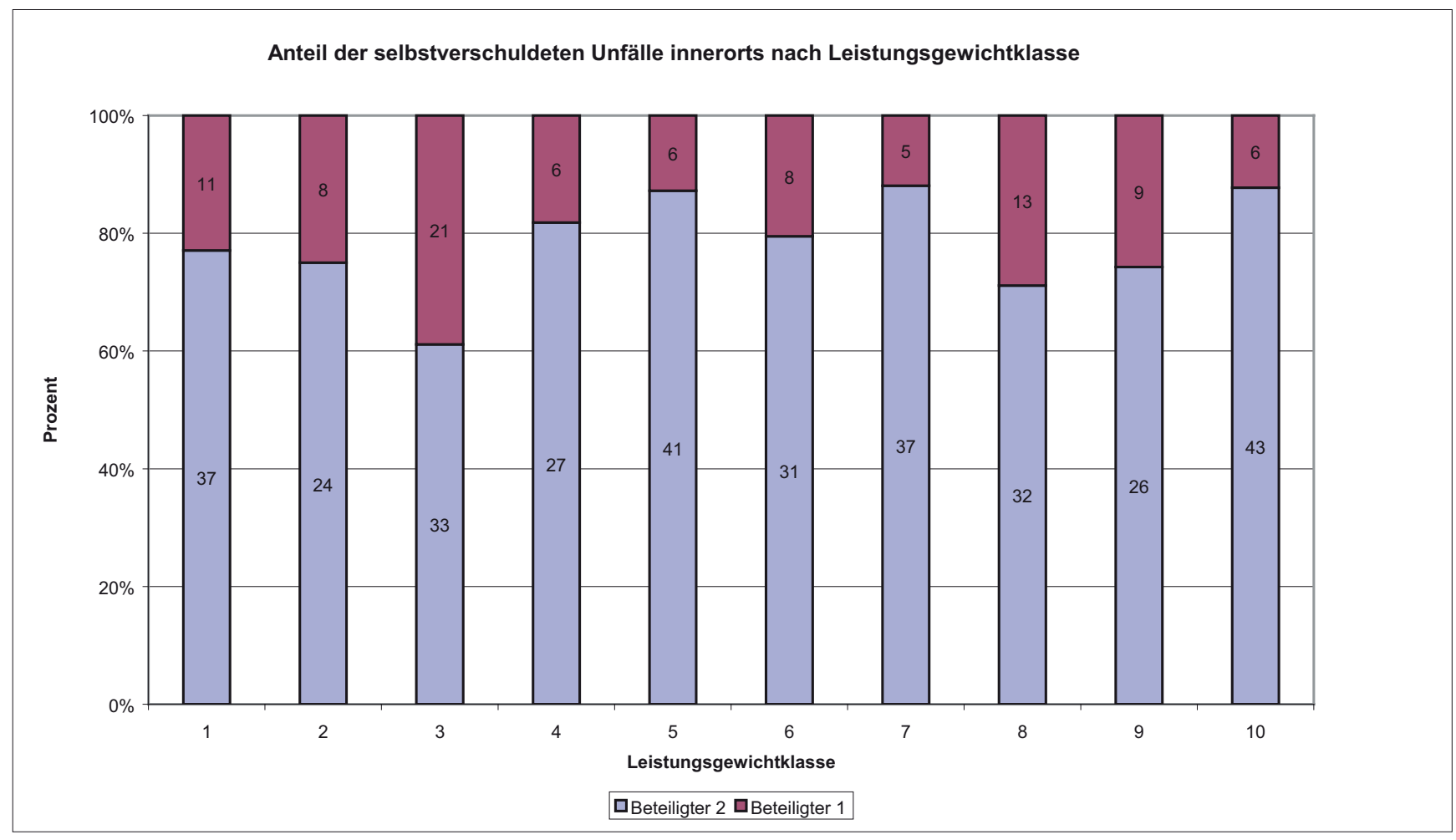

Abbildung 6-23: Anteil der selbstverschuldeten Unfälle innerorts nach Leistungsgewicht 
ligung insgesamt. Der Unterschied resultiert aus den nicht repräsentativen GDV-Daten (vgl. Beginn Kapitel 6). Die Anteile der selbst verschuldeten Unfälle sind bei den GDV-Daten im Vergleich zur Bundesstatistik wie folgt verteilt: Bei den 53 tödlichen Außerortsunfällen liegt die Rate des Selbstverschuldens bei $52 \%$, wenn man die Alleinunfälle mit einbezieht. Über alle 431 Außerortsunfälle liegt der Anteil der selbstverschuldeten Unfälle bei $40 \%$. Werden vergleichsweise die 423 auf Basis der amtlichen Statistik untersuchten Außerortsunfälle in Sachsen betrachtet, ergeben sich 69 \% der Unfälle mit dem PTW-Fahrer als Hauptverursacher. Über die Hälfte davon sind Alleinunfälle (55\%). Bei Auswertung allein der Unfälle mit Getöteten resultiert ein Anteil von $68 \%$ mit dem PTW als Hauptverursacher, $53 \%$ sind davon Alleinunfälle.
Zur Klärung des Einflusses von Leistungsgewicht auf das Unfallereignis kann eine differenzierte Aufstellung der selbstverschuldeten Unfälle über die Leistungsgewichtsklassen beitragen (Abbildung 6-23).

Ohne große Auffälligkeiten schwingt der Wert des Selbstverschuldens innerorts um $20 \%$, steigt außerorts jedoch auf Werte zwischen $10 \%$ und $50 \%$ (Abbildung 6-24).

Unter den selbstverschuldeten Unfällen mit Todesfolge ist jedoch ein Einfluss des Leistungsgewichtes auf den Unfallverlauf zu erkennen (Abbildung 6-25). Über 80 \% der selbstverschuldeten Unfälle außerorts unterhalb eines Leistungsgewichtes von $2,6 \mathrm{~kg} / \mathrm{kW}$ sind tödlich. Nur zwei der 53 tödlichen Unfälle außerorts sind Alleinunfälle (3,8\%).Dies spiegelt die Varianzanalyse wider (vgl. 6.2.5).

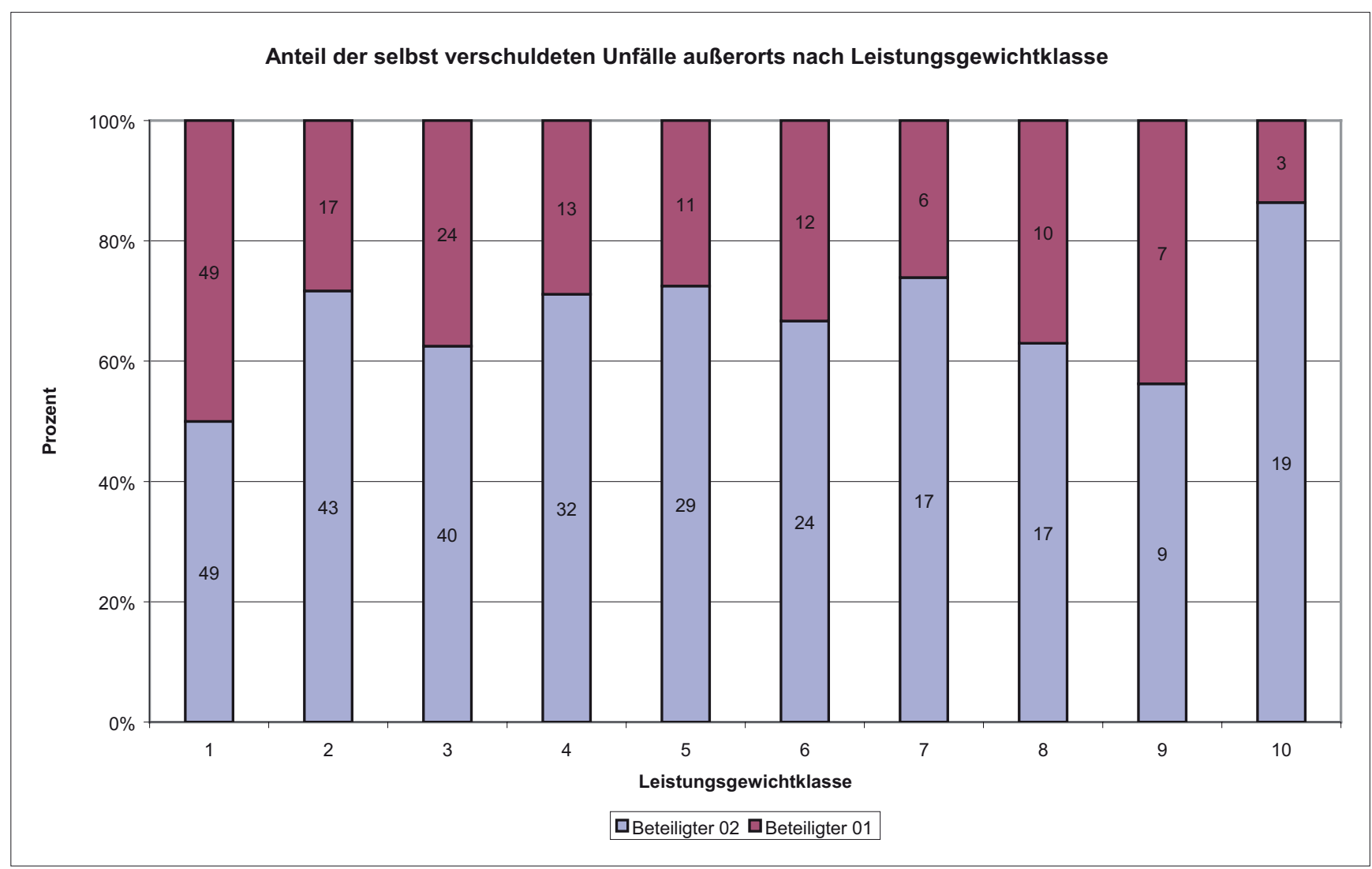

Abbildung 6-24: Anteil der selbstverschuldeten Unfälle außerorts nach Leistungsgewicht 


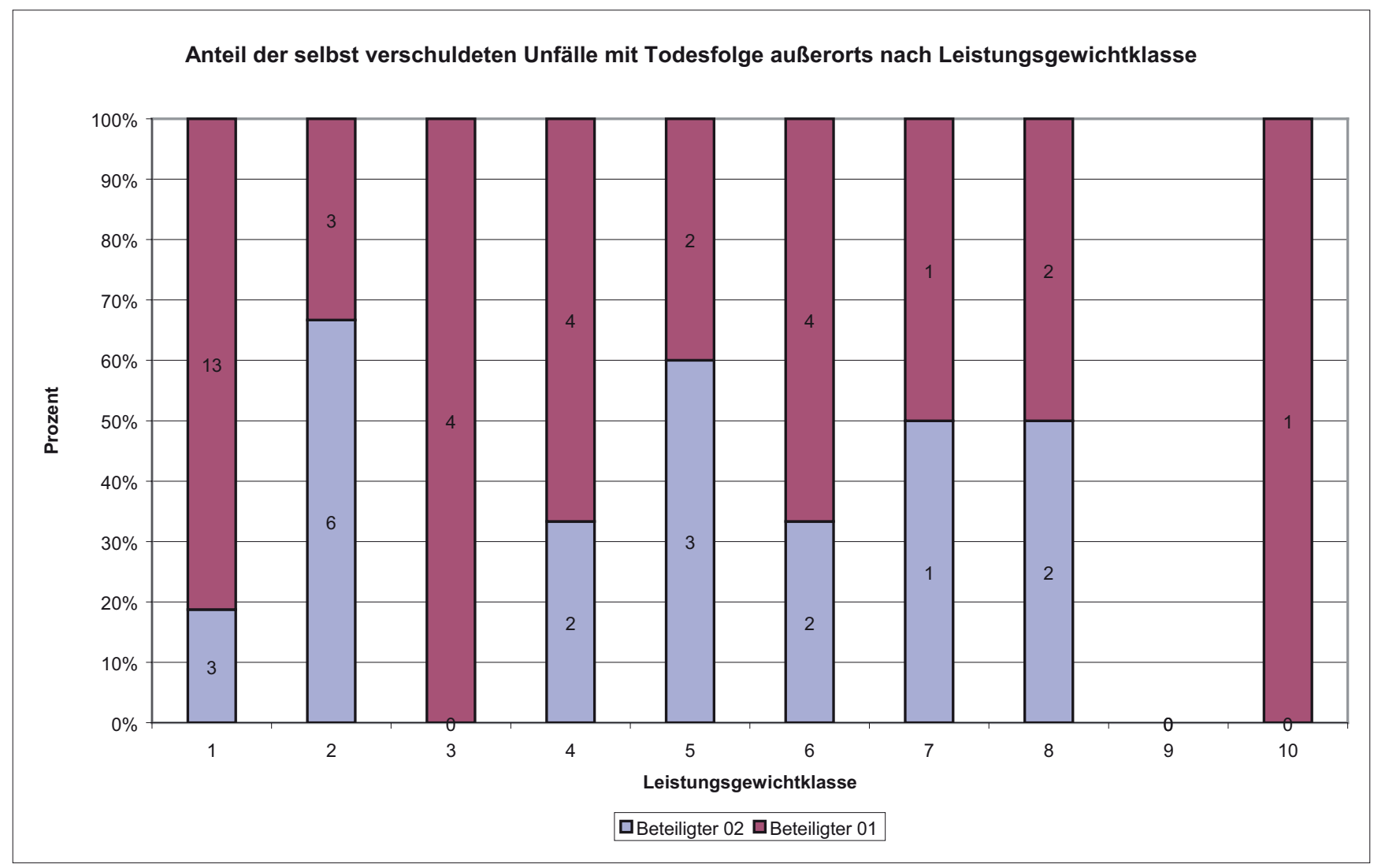

Abbildung 6-25: Anteil der selbstverschuldeten Unfälle mit Todesfolge außerorts nach Leistungsgewicht

\subsubsection{Zusammenfassung}

Die Analyse der GDV Unfalldatenbank zeigt, dass technische Eigenschaften am Motorrad nicht unbedingt Einfluss auf die Verletzungsschwere eines Motorradfahrers haben. So mindert z. B. das Vorhandensein von Seitenkoffern an den Einspurfahrzeugen nicht signifikant die Verletzungsschwere der unteren Extremitäten. Mit und ohne Koffer haben die betroffenen Motorradfahrer zu ca. $20 \%$ MAIS $3+$ Verletzungen an unteren Extremitäten. Der zusätzliche Freiraum im Falle eines Sturzes hat so keinen positiven Einfluss auf die Verletzungsschwere. Auch hat die Lenkerform keinen direkten Einfluss auf die Verletzungsschwere. Fahrer eines Motorrades mit einem niedrigen Lenker haben zwar eine höhere Verletzungsschwere als Fahrer von Motorrädern mit mittel hohen Lenkern, doch ist in diesem Fall nicht die reine Lenkerhöhe ausschlaggebend, sondern das Leistungsgewicht des Fahrzeuges. Der Korrelationskoeffizient zwischen Leistungsgewichtklasse und Lenkerhöhe liegt hochsignifikant bei 0,482 (Tabelle 6-7).

\begin{tabular}{|ll|r|r|}
\hline & & LGKlasse & Lenkertyp \\
\hline LGKlasse & Pearson Correlation & 1 &, $482\left(^{* *}\right)$ \\
& Sig. (2-tailed) & &, 000 \\
$\mathrm{~N}$ & 879 & 851 \\
Lenkertyp & Pearson Correlation &, $482\left(^{* *}\right)$ & 1 \\
& Sig. (2-tailed) &, 000 & \\
$\mathrm{~N}$ & 851 & 1103 \\
\hline
\end{tabular}

${ }^{* *}$ Correlation is significant at the 0.01 level (2-tailed).

\section{Tabelle 6-7: Korrelation zwischen Höhe des Lenkers und des Leistungsgewichts}

Der Einfluss des Leistungsgewichts auf die Verletzungsschwere ist in der Stichprobe hochsignifikant und mit $16 \%$ genauso hoch wie der Einfluss der Unfallart, die jedoch nicht beeinflusst werden kann. Dabei sind nicht nur die reine Leistung und Masse zu betrachten, sondernn auch jene Faktoren, die ein hohes Beschleunigungsver- 
mögen ermöglichen. Ist die Verkehrsdichte außerorts geringer, so kann in kürzerer Zeit eine höhere Geschwindigkeit erreicht werden. Auch hier sind im Mittel die Verletzungsschweren höher.

Als „Faustregel“ gilt, dass Unfälle außerorts einen im Mittel um eins höheren MAIS-Wert haben als Unfälle innerorts. Zudem steigt die Verletzungsschwere, wenn der Motorradfahrer in die Fahrerseite des Unfallgegners fährt auch um ca. einen MAIS-Wert im Mittel (vgl. 6.2.2). Die geringsten Verletzungsschweren sind so innerorts bei Unfällen mit mehr als einem Beteiligten zu verzeichnen, bei denen es zu keiner Kollision kommt.

Der häufigste Unfallablauf bei Unfällen mit mehr als einem Beteiligten ist die stabile Fahrt auf der Geraden oder in einer Kurve mit der aufrechten Kollision im Anschluss. Rutscht das Motorrad jedoch vor der Kollision weg, so ist die Verletzungsschwere höher, weil weniger kinetische Energie abgebaut wird, als wenn der Fahrer eine Gefahrenbremsung vollführte. Bei der aufrechten Kollision sind Kopf und Thorax am höchsten belastet, da der Motorradfahrer mit häufig auf die steife Dachkante prallt. Auch ein Überfliegen des Unfallgegners durch den Motorradfahrer führt zu geringeren Verletzungsschweren. Der Weg des Energieabbaus ist dabei wesentlich länger und das Belastungsniveau kann somit geringer ausfallen. Der gezielte Überflug ist jedoch nicht zu favorisieren, da nie sichergestellt werden kann, ob ein Überflug gelingt und hinter dem Unfallgegner genügend Platz zur Verfügung steht. Alleinunfälle sind in der Datenbasis weniger schwerwiegend. Sie verlaufen großteils ohne Kollision und so kann die kinetische Energie durch ungestörtes Rutschen abgebaut werden.

Der Motorradtyp als technisches Merkmal des Motorrades hat wiederum keinen signifikanten Einfluss auf die Höhe der MAIS-Werte. Fahrer von Sportmaschinen sind im allgemeinen schwerer betroffen, doch kann dies wiederum durch die niedrigeren Leistungsgewichte in dieser Motorradklasse erklärt werden. Sportmaschinen haben in der Datenbasis eine mittlere Leistung von ca. 73 kW.
Chopper haben dagegen durchschnittlich eine Leistung von $31,5 \mathrm{~kW}$.

Im Gegensatz zum Pkw sind so genannte Diskounfälle bei Motorradunfällen praktisch nicht existent. Motorradfahrer steigen fast nie unter dem Einfluss von Alkohol auf ein Zweirad (vgl. 6.2.3).

\subsection{Auswertung der Daten der Motor- radumfrage}

Zur Ermittlung des Fahrverhaltens von Motorradfahrern wurde ein Fragebogen (Anlage 09) entwickelt, der in ca. 40 Punkten Fragen zur Einstellung zum Motorradfahren, zu begangenen Ordnungswidrigkeiten im Straßenverkehr und erlittenen Unfällen, sowie den daraus resultierenden Verletzungen stellt. In Zusammenhang mit den technischen Eigenschaften der jeweiligen Fahrzeuge und den individuellen personenbezogenen Unterschieden der dazugehörigen Fahrzeugführer soll die empirische Datenbasis Aufschluss darüber geben, inwieweit es Auffälligkeiten bei bestimmten Personengruppen, Fahrzeugkategorien oder kategorieübergreifenden Fahrzeugeigenschaften auch mit auftretenden Interaktionseffekten ${ }^{4}$ gibt.

Die Datenerhebung fand im Zeitraum vom 19.01.2007 bis zum 18.02.2007 ausschließlich im Internet unter www.motorradumfrage.de per Online-Befragung statt. Der Aufruf dazu wurde in dem 14-tägig erscheinenden Motorradmagazin "MOTORRAD“, Ausgabe 03/07 (Anlage 09), mit einem doppelseitigen Hinweis zum Projekt gedruckt. Bei einer Auflage von 146.000 Exemplaren und einer Teilnehmerzahl von 6.879 teils unvollständig beantworteten Fragebögen, ergibt sich eine Rückmeldequote von 4,7\%. Mit den 5.297 vollständig ausgefüllten Fragebögen ergibt sich immer noch eine Rückmeldequote von $3,6 \%$.

Der recht hohe Anteil kann durch das von der Firma BMW-Motorrad gesponserte Sicherheitssystem für drei ausgeloste Teilnehmer erklärt werden, welches zum Sai- 
sonstart 2007 in den Markt eingeführt wurde. Die drei Neck Brace Halskrausen haben einen Gesamtwert von ca. $1.500 €$ im Verkauf.

Von den Motorradfahrern der 5.297 vollständigen Datensätzen haben 2.983 Personen bereits einen Unfall gehabt. Dies entspricht einem Anteil von 56,3\%. Bei einem Frauenanteil von 5,53\% $(n=296)$ haben nur $n=105$ weibliche Motorradfahrer einen Unfall erlitten. Dies entspricht einem Anteil von 35,8\%.

Die Befragung ermöglicht eine Aussage zur polizeilichen Dunkelziffer: Von den 2.555 angegebenen Unfällen, zu denen nähere Angaben gemacht wurden, erfolgte in 49 \% der Fälle keine polizeiliche Aufnahme. 69 \% davon sind Alleinunfälle. Werden allein die Unfälle betrachtet, bei denen der Motorradfahrer eine Verletzung davon trug, resultiert eine Dunkelziffer von $33 \%$. Diese sinkt bei alleiniger Betrachtung der Unfälle mit schwerverletztem Motorradfahrer auf $14 \%$. Anlage 11 zeigt die Befragungsergebnisse zur Unfallaufnahme in Abhängigkeit der Unfallschwere und der Anzahl der Beteiligten.

\subsubsection{Altersverteilung}

Betrachtet man die Altersverteilung (Abbildung 6-26) der Personen, die an der Befragung teilgenommenen haben, so ist zu erkennen, dass auch hier der allgemeine Trend zum immer älter werdenden Motorradfahrer bestätigt wird.

Ein Großteil der Motorradfahrer ist zwischen 35 und 50 Jahren alt. Betrachtet man die Altersverteilung der Motorradfahrer mit Unfall in der Stichprobe, so ist zu erkennen, dass keine Altersgruppe besonders über- oder unterrepräsentiert ist.

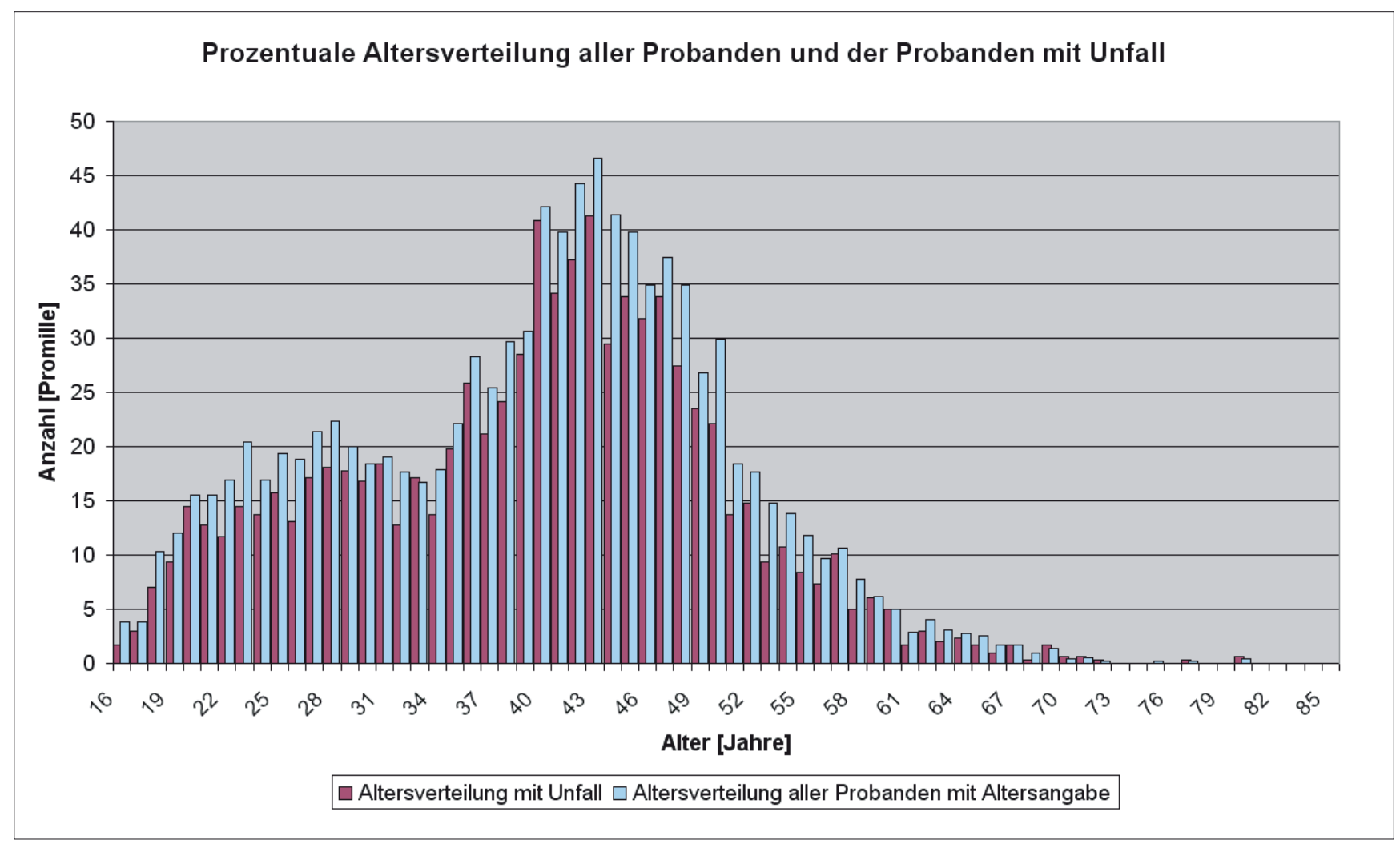

Abbildung 6-26: Altersverteilung der Teilnehmer an der Befragung 


\subsubsection{Verteilung der Motorradtypen in der Umfrage}

Auffällig bei der Verteilung der Motorradtypen in der Umfrage (Abbildung 6-27) ist, dass knapp über 40 \% der Motorräder Sportmaschinen sind. Mit den Naked Bikes zusammen bilden sie so über zwei Drittel in den erhobenen Daten. Dies kann dadurch erklärt werden, dass bei diesen Fahrergruppen das Sicherheitsbewusstsein höher ist als bei Chopper- und Rollerfahrern. Die Erklärung wird auch durch das Nutzungsverhalten von Sicherheitskleidung der Fahrer gestützt (vgl. 6.3.8). Auch kann der ausgeschriebene Preis des Gewinnspiels im Rahmen der Umfrage dazu geführt haben, dass prinzipiell eher die Personen teilgenommen haben, die mit einer Neck Brace Motorrad fahren würden. Chopper- und Rollerfahrer gehören weniger zu diesen Personengruppen.

Betrachtet man die technischen Eigenschaften der Motorräder, so kann man zwischen den verschiedenen Fahrzeugen folgende Unterscheidungen beobachten (Abbildung 6-28). Sportmaschinen erreichen gegenüber den Choppern mit einem durchschnittlichen Wert von $88 \mathrm{~kW}$ fast die doppelte Leistung. Entsprechend verhält es sich mit der Drehzahl, aber mit einer Masse von $217 \mathrm{~kg}$ sind sie im Schnitt fast $40 \mathrm{~kg}$ leichter. Der durchschnitt-

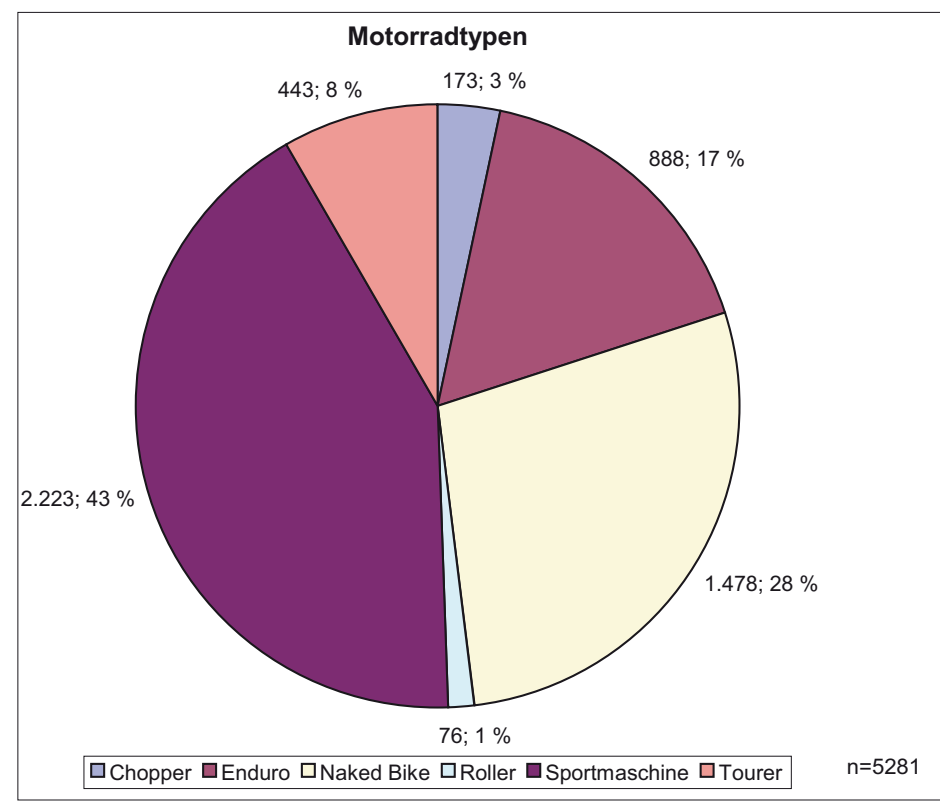

Abbildung 6-27: Verteilung der Motorradtypen in der Umfrage liche Hubraum erreicht bei den Choppern mit über einem Liter Hubraum ein Maximum. Der hohe Hubraumwert für die Roller ist durch den großen Anteil von Kraftrollern über $125 \mathrm{~cm}^{3}$ zu erklären. Bauartbedingt weisen die Tourenmodelle das höchste durchschnittliche, voll getankte Leergewicht auf. Die Werte für Enduros liegen so hoch, da in dieses Segment die meist verkaufte Maschine in Deutschland fällt. Die BMW GS besitzt einen Zweizylinder Boxermotor von 1.000 bis $1.200 \mathrm{~cm}^{3}$ und wiegt über $200 \mathrm{~kg}$.

\subsubsection{Motivation zum Motorradfahren}

Knapp die Hälfte der Antworten auf die Frage, aus welchem Grund Motorrad gefahren wird, waren auf die Fahrdynamik des Motorrades ausgerichtet. So wurde Kurvenfahrt und Beschleunigung als Hauptmotivationen zum Motorradfahren genannt. Die erzielbare Geschwindigkeit selbst ist mit $8 \%$ von untergeordneter Rolle. Eine zweite Hauptmotivation des Motorradfahrens ist die empfundene Freiheit als Aufsasse eines Zweirades (Abbildung 6-29).

Betrachtet man die Aussagen jedoch differenziert über die Motorradtypen (Abbildung 6-30), so wird ersichtlich, dass Fahrer der unterschiedlichen Klassen (vgl. 1.2) andere Anforderungen an das Fortbewegungsmittel stellen und gerade daher auch den bestimmten Motorradtypen benutzen. Chopperfahrer schätzen besonders die Freiheit auf einem Motorrad, wogegen Sportmaschinenfahrer im Vergleich zu den anderen Segmenten verstärkt die Sportlichkeit und Geschwindigkeit ihrer Motorräder schätzen. Die auffälligste Abweichung ist im Rollersegment zu finden. Diese Personengruppe hat besonders den Kostenund Größenvorteil in den Vordergrund gestellt (vgl. 6.3.4) (Abbildung 6-30).

\subsubsection{Nutzungsverhalten}

Das praktische Denken der Rollerfahrer spiegelt sich auch im Nutzungsverhalten (Abbildung 6-31) wieder. Bei keinem Motorradtyp wird das Fahrzeug so intensiv auch für den Alltag genutzt. Über 50 \% der Motorradnutzer fahren ausschließlich zum Vergnügen in der Freizeit. Nur im Rol- 


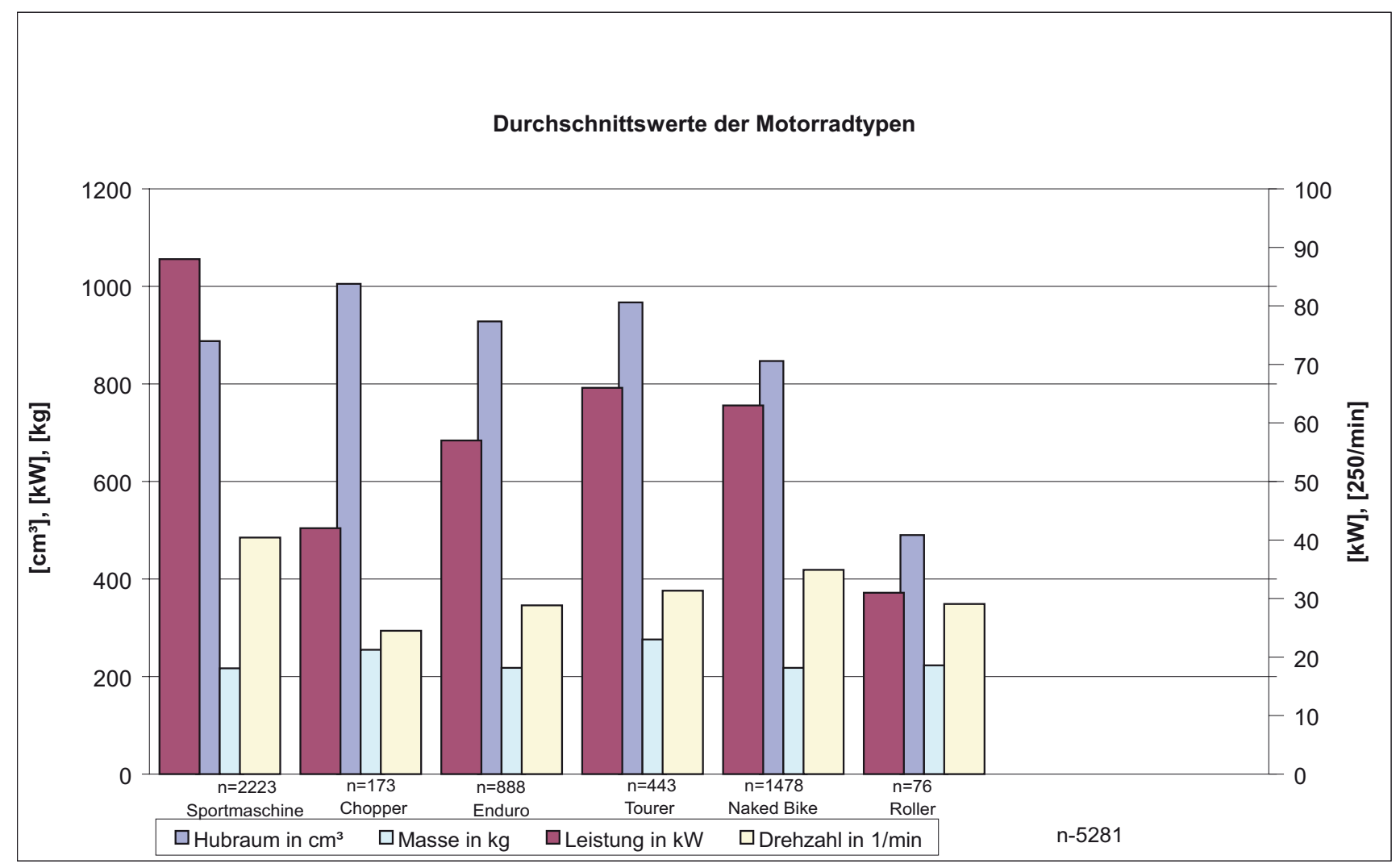

Abbildung 6-28: Durchschnittliche Fahrzeugdaten über die einzelnen Motorradtypen

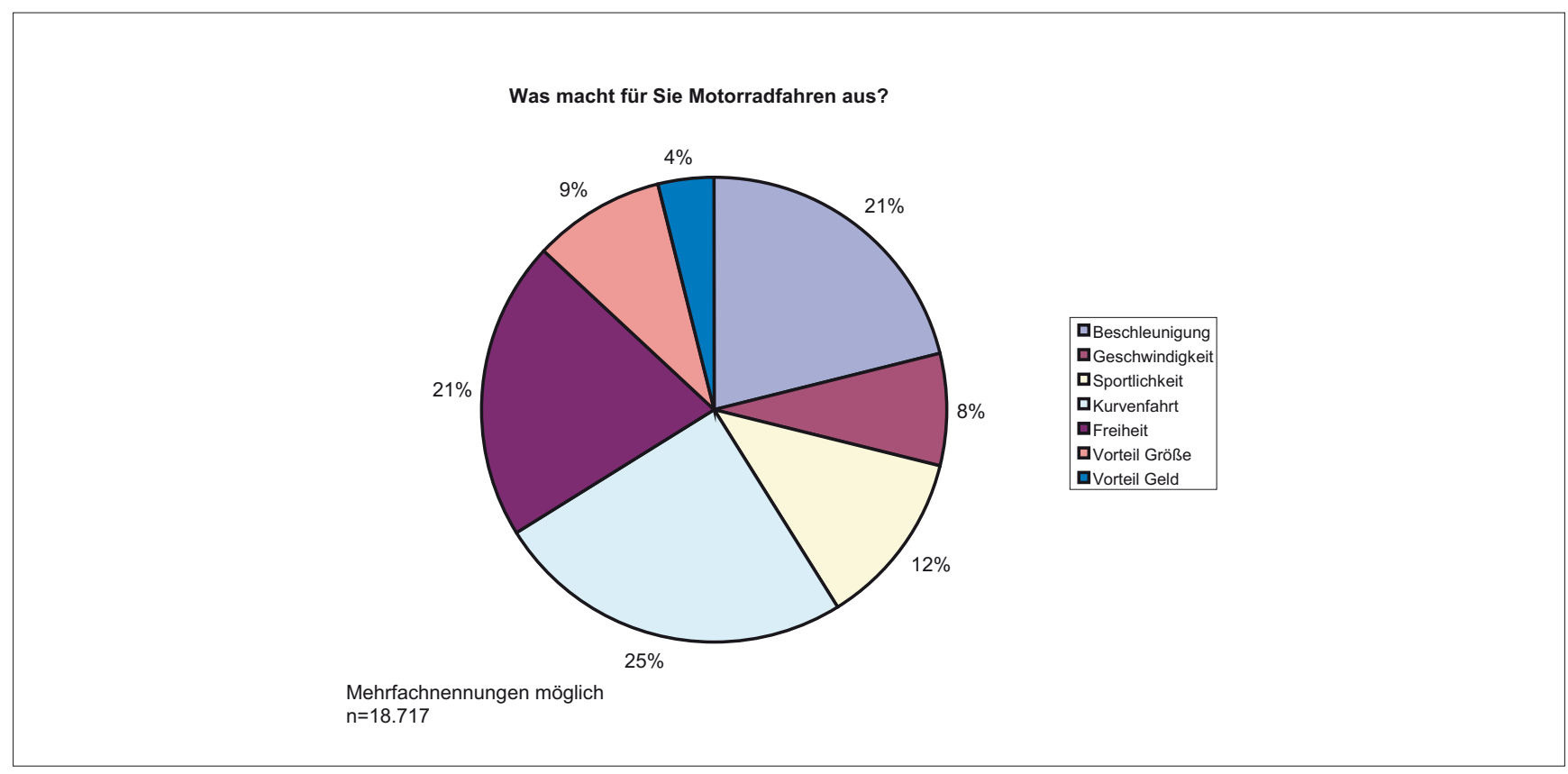

Abbildung 6-29: Motivation zum Motorradfahren 


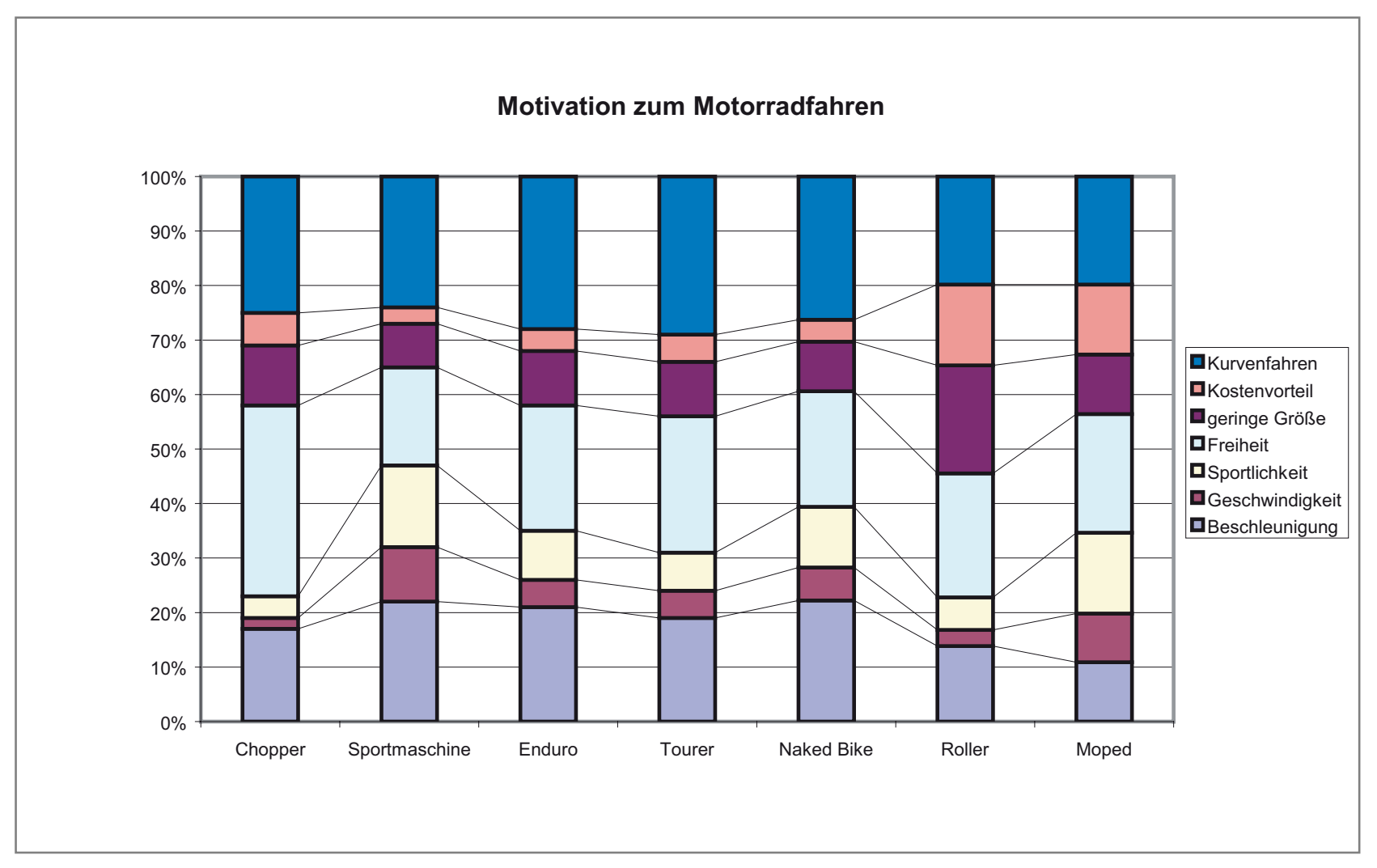

Abbildung 6-30: Differenzierung der Motivation zum Motorradfahren nach Motorradtyp

lersegment ist dieser Anteil auf 15 \% begrenzt. Über $70 \%$ geben an, dass sie ihr Zweirad im Alltag und zur Freizeit nutzen und knapp $10 \%$ ausschließlich im Alltag.

Das Nutzungsverhalten in Abhängigkeit von der Witterung unterstreicht die pragmatische Haltung der Rollerfahrer (Abbildung 6-32). Von schlechtem Wetter zeigen sich die wenigsten Rollerfahrer beeindruckt. Über $50 \%$ geben an, dass sie häufig bis immer auch bei widrigen Witterungsverhältnissen dieses Fortbewegungsmittel nutzen. Dies kann verschiedene Gründe haben, die vom leicht besseren Wetterschutz bis zum Mangel eines anderen Alltagsfahrzeugs reichen. Da in anderen Motorradsegmenten überwiegend in der Freizeit gefahren wird, kann davon ausgegangen werden, dass die jeweiligen Fahrer zusätzlich einen Pkw besitzen, auf den sie bei schlechtem Wetter umsteigen.
Auffällig ist zudem, dass $30 \%$ der Chopperfahrer ausgewiesene „Schönwetterfahrer" sind. Darüber lässt sich eine Hypothese aufstellen, die jedoch an dieser Stelle nicht bewiesen werden kann. Bei Choppern handelt es sich großteils um sehr kostspielige Fahrzeuge mit viel Chrom und schmuckvollen Verzierungen. Ein Teil der Philosophie in diesem Segment ist es sein Fahrzeug zu individualisieren um aus der Masse herauszustechen. Dies bedarf einer intensiven Pflege des kostbaren Gefährts, wodurch es bei schlechtem Wetter bevorzugt nicht verwendet wird.

Im Winter (Abbildung 6-33) melden zudem die Halter von Rollern ihr Fahrzeug am seltensten ab. Nur 10 \% verzichten darauf ihr Fahrzeug im Winter angemeldet zu behalten. Auch geben knapp $30 \%$ an, dass sie im Winter immer fahren. 


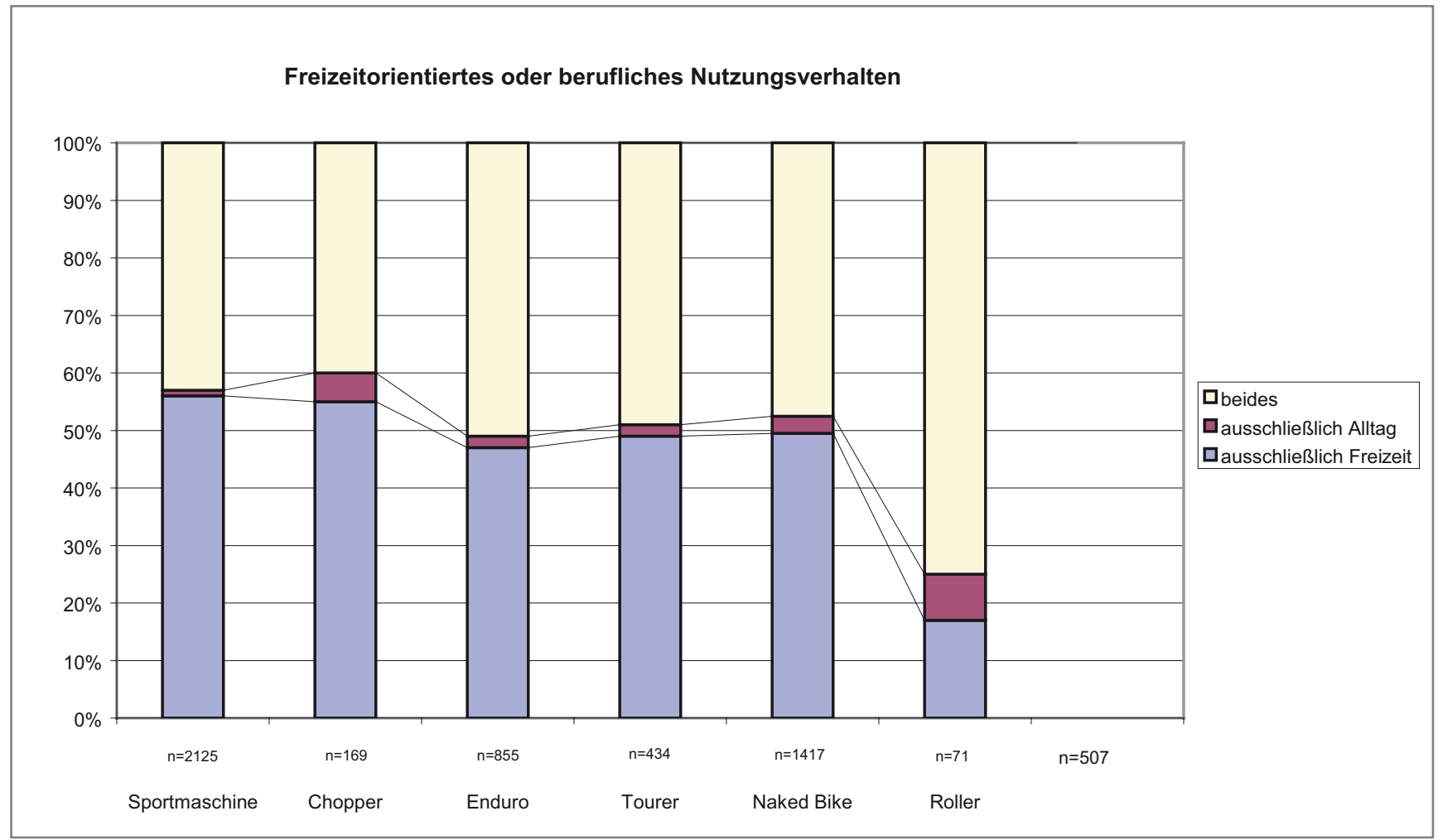

Abbildung 6-31: Nutzungsverhalten der Motorradfahrer über die unterschiedlichen Motorradtypen

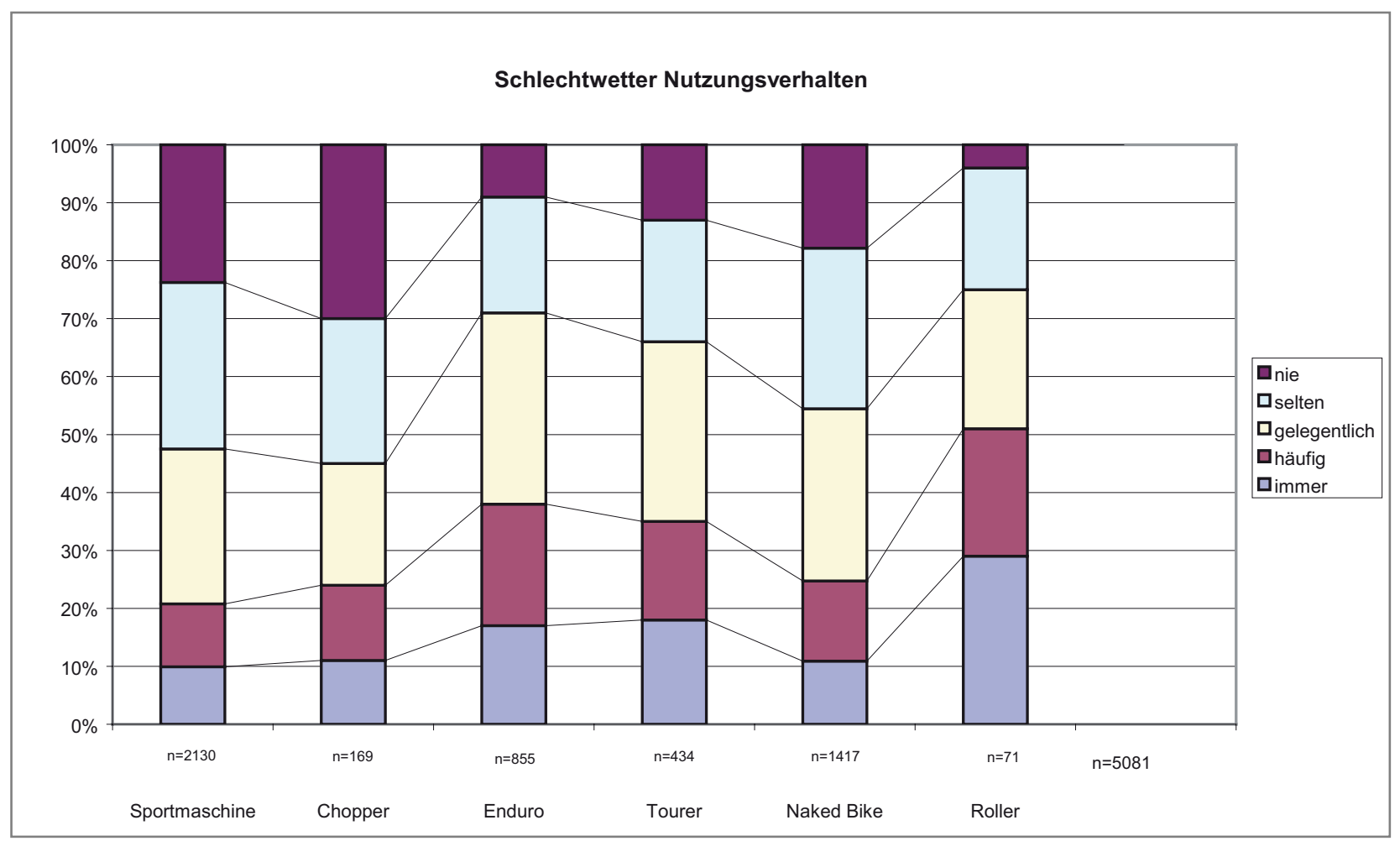

Abbildung 6-32: Nutzungsverhalten bei schlechtem Wetter 
Als weiteres Motorradsegment fällt das der Enduros auf. Über 60 \% geben an, dass sie zumindest auch selten im Winter mit ihrem Fahrzeug unterwegs sind.

Die spezialisierten Reifen der Sportmaschinen und zum Teil auch die der Naked Bikes benötigen eine Mindesttemperatur für eine ausreichende Haftung auf der Fahrbahnoberfläche. Dadurch ist es zu verstehen, dass diese Fahrzeuge bei niedrigen Temperaturen in den Wintermonaten seltener bewegt werden.

Da Motorräder größtenteils nicht über Typklassen versichert sind, sondern die Versicherungsprämie aufgrund der Motorleistung festgelegt wird, kann der hohe Anteil von knapp $40 \%$ abgemeldeten Sportmaschinen im Winter erklärt werden. Bei der hohen Leistung von durchschnittlich 88 kW ist ein größerer Kostenvorteil im Vergleich zu den anderen Motorradsegmenten durch die Abmeldung im Winter gegeben.

\subsubsection{Selbsteingeschätztes Fahrkönnen und Fahrstil}

Auf einer äquidistanten Schätzskala von 0 bis 4 sollten die Probanden ihren Fahrstil von sportlich bis bedacht für den Fahrstil und von Anfänger bis geübter Fahrer für das Fahrkönnen einordnen. Über die einzelnen Motorradsegmente in Abhängigkeit des Fahreralters kann beobachtet werden, dass sich junge Fahrer bewusst sind, das Fahrkönnen eines Anfängers zu haben (Abbildung 6-34). Bei den 16- und 17jährigen Sportmaschinenfahrern liegt der Wert bei 1,72. Der allgemeine Trend über die unterschiedlichen Motorradsegmente ist, dass ältere Fahrer ihr Fahrkönnen als geübter einschätzen. Unterschiede zwischen den Motorradsegmenten sind nicht zu verzeichnen.

Bei den Fahrstilen jedoch fällt auf, dass besonders die Sportmaschinenfahrer sich als sehr "sportlich“ einschätzen und nicht als „,bedacht“. Besonders die jungen Fahrer, die sich zuvor als Anfänger bezeichnet haben, geben an sportlich zu fahren.

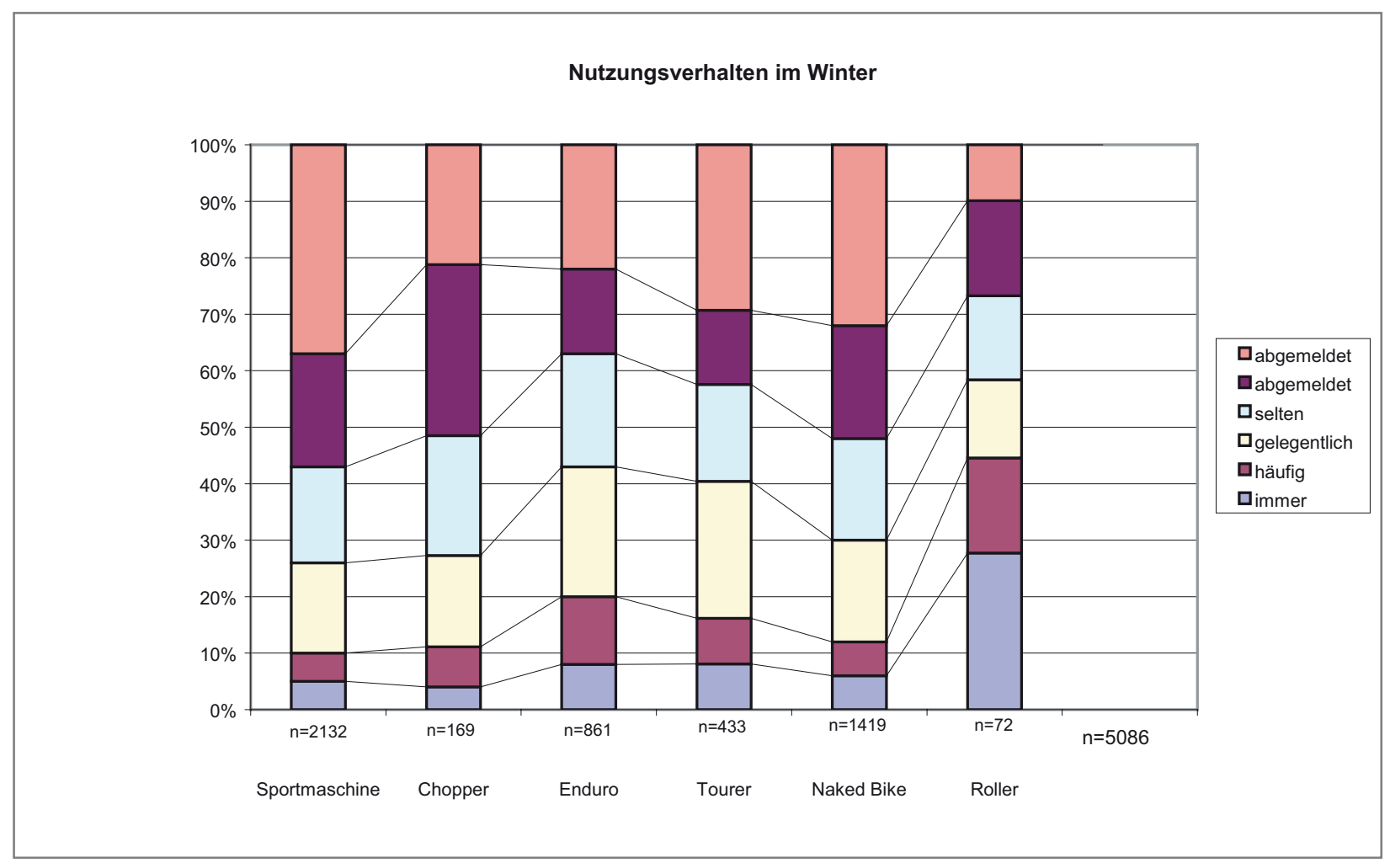

Abbildung 6-33: Nutzungsverhalten im Winter 
Auch in den höheren Altersklassen der Sportmaschinenfahrer ändert sich der sportliche Fahrstil nicht. Erst ab 55 Jahre wird etwas bedachter gefahren. In den übrigen Motorradklassen ist ein stärkerer Trend zur bedachten Fahrweise im höheren Alter gegeben.

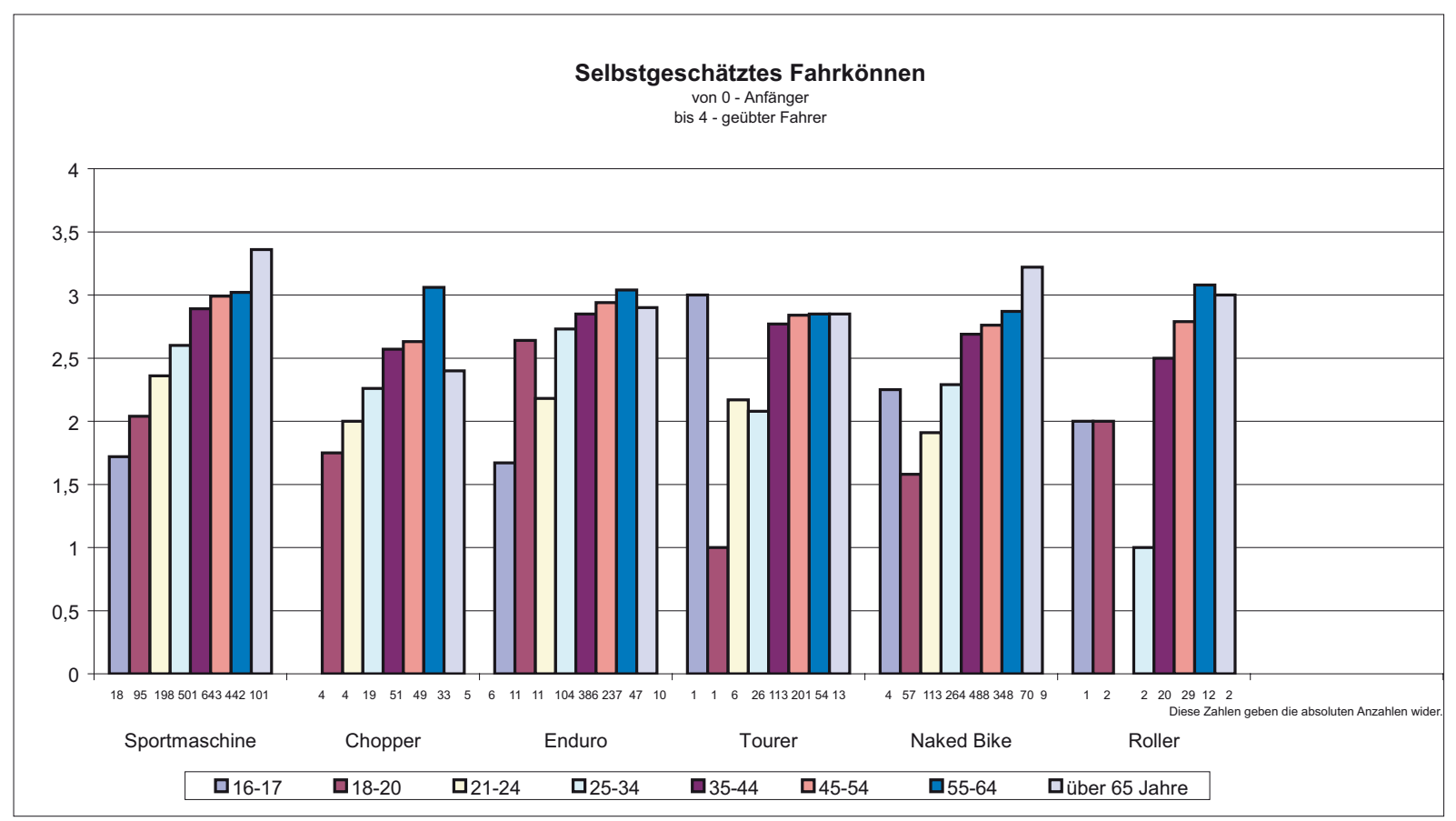

Abbildung 6-34: Selbstgeschätztes Fahrkönnen

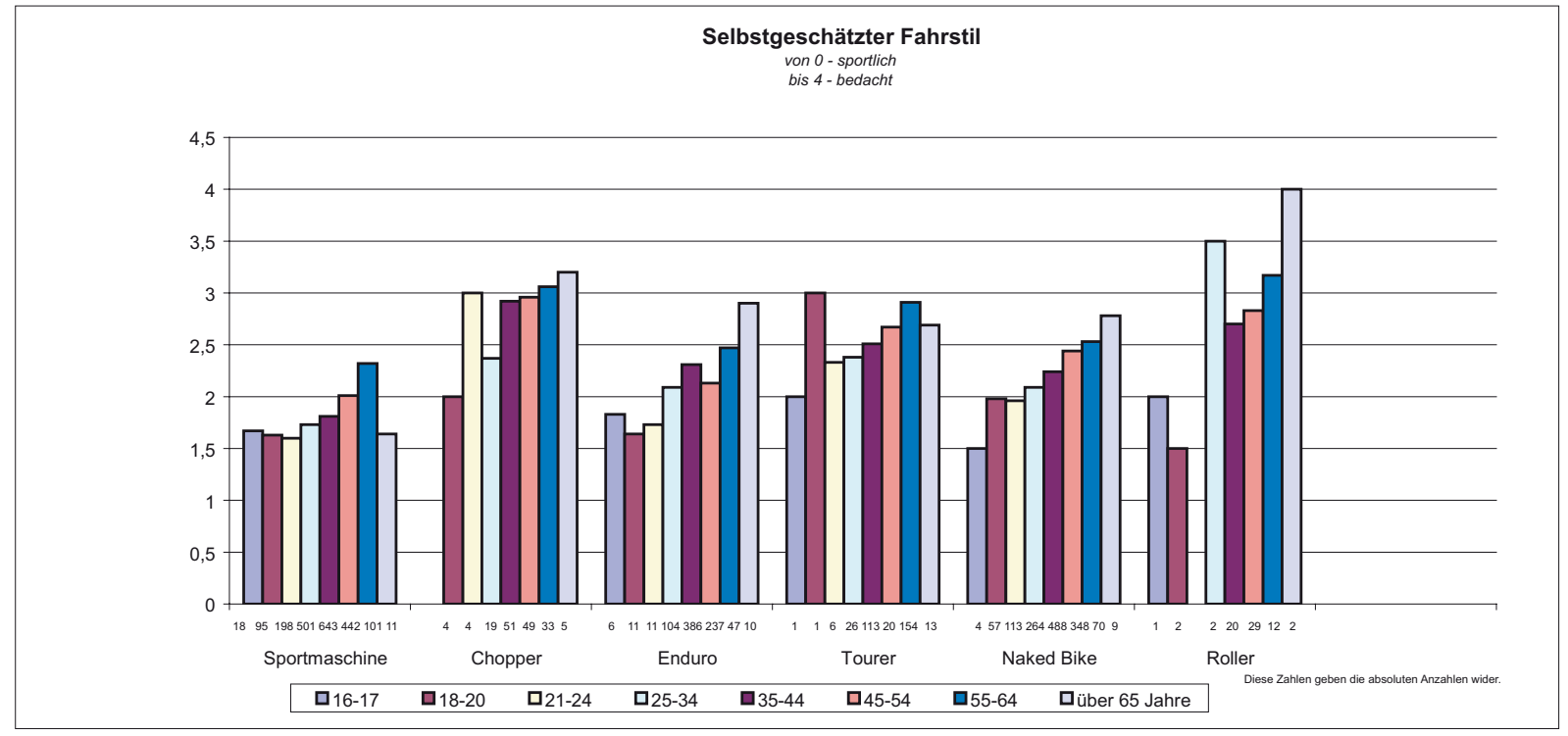

Abbildung 6-35: Selbstgeschätzter Fahrstil 


\subsubsection{Straßennutzungsverhalten}

Unter den verschiedenen Motorradsegmenten gibt es keine großen Abweichungen im Straßennutzungsverhalten (Abbildung 6-36 bis Abbildung 6-41). Enduros, Sportmaschinen, Naked Bikes und Tourer werden nur zu einem Viertel innerhalb geschlossener Ortschaften bewegt. Die Fahrten außerorts teilen sich nach ca. 85 \% Landstraße und $15 \%$ Autobahn auf.

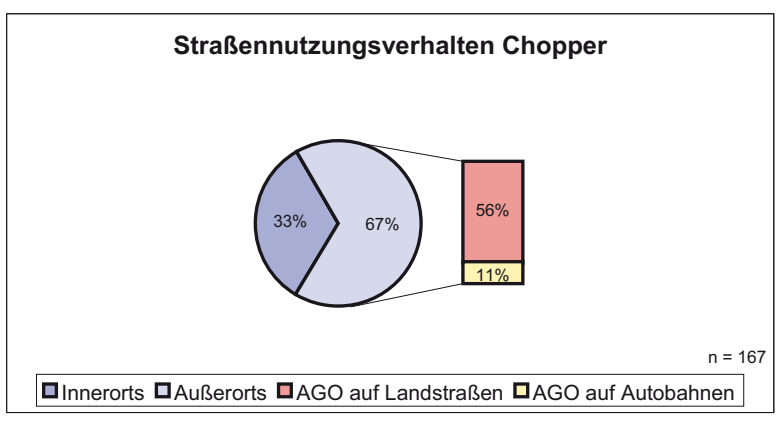

Abbildung 6-36: Straßennutzungsverhalten Chopper

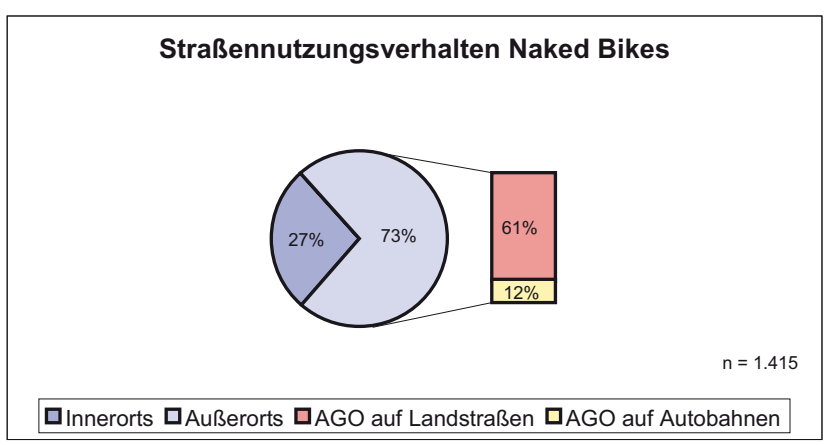

Abbildung 6-38: Straßennutzungsverhalten Naked Bikes

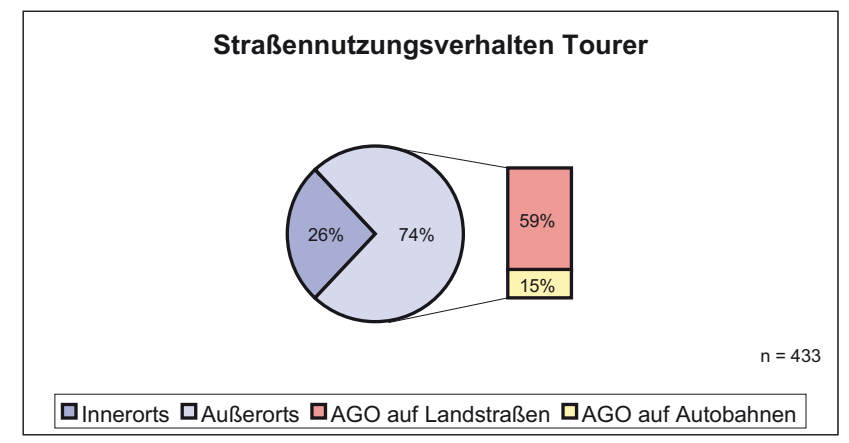

Abbildung 6-40: Straßennutzungsverhalten Sportmaschinen
Abweichungen können bei Choppern und Rollern beobachtet werden. Hier wird über ein Drittel der Fahrten in der Stadt absolviert. Bei den Rollern werden sogar 22 \% der außerörtlichen Fahrten auf Autobahnen gefahren. Dies zeigt wieder den praktischen Anspruch der Rollerfahrer. Nicht die Fahrt ist das Ziel, sondern das Ankommen. Die starke Autobahnbenutzung ist auch nur durch die große Anzahl an Kraftrollern in der Umfrage möglich, die auf Autobahnen fahren dürfen.

\section{Straßennutzungsverhalten Enduro}

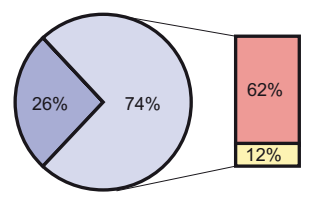

$n=859$

口Innerorts $\square$ Außerorts $\square$ AGO auf Landstraßen DAGO auf Autobahnen

\section{Abbildung 6-37: Straßennutzungsverhalten Enduro}

Straßennutzungsverhalten Roller

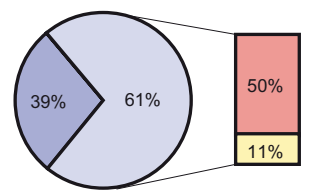

$\mathrm{n}=71$

口Innerorts $\square$ Außerorts $\square$ AGO auf Landstraßen $\square$ AGO auf Autobahnen

Abbildung 6-39: Straßennutzungsverhalten Roller

Straßennutzungsverhalten Tourer

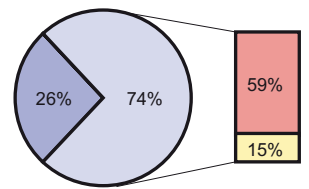

$n=433$

$\square$ Innerorts $\square$ Außerorts $\square$ AGO auf Landstraßen $\mathbf{D A G O}$ auf Autobahnen 


\subsubsection{Verkehrsverstöße}

Um eine Korrelation zwischen Verkehrsauffälligkeit und Unfallhäufung/-schwere aufstellen zu können, wurden die Motorradfahrer auch nach ihren Verkehrsverstößen befragt. Um unter den verschiedenen Motorradsegmenten einen Vergleich zu ermöglichen, wurden die jeweiligen Einzelfälle auf die Antworthäufigkeit in dem Segment in der Umfrage bezogen. So ist zu erkennen, dass Fahrer von Sportmaschinen ein wesentlich höheres Risiko in Kauf nehmen, wegen eines Verkehrsverstoßes belangt zu werden (Abbildung 6-42).

Dabei werden größtenteils Regelverstöße wegen zu schnellen Fahrens verübt (Abbildung 6-43).

Danach folgen Verstöße auf Grund von falschem Überholen und Mängel an den Fahrzeugen, die nach Sportma- schinen und Naked Bikes mit abgefahrenen Reifen und zu lauten Auspuffanlagen bei Choppern gegliedert werden können.

Die Verstöße wegen zu schnellen Fahrens und falschen Überholvorgängen können ein Zeichen dafür sein, dass besonders leistungsstarke Motorräder den Fahrer dazu verleiten, nicht jederzeit den Verkehrsregeln entsprechend zu fahren. Dieses Phänomen kann losgelöst von den Fahrzeugtypen auf das Leistungsgewicht bezogen beobachtet werden (Abbildung 6-44). Je geringer die Masse bezüglich der Leistung, desto eher wird der Fahrer durch Geschwindigkeits- oder Überholverstöße auffällig. Das starke Schwanken bei den höheren Leistungsgewichten ist durch die geringen Anzahlen innerhalb der Gruppen zu erklären.

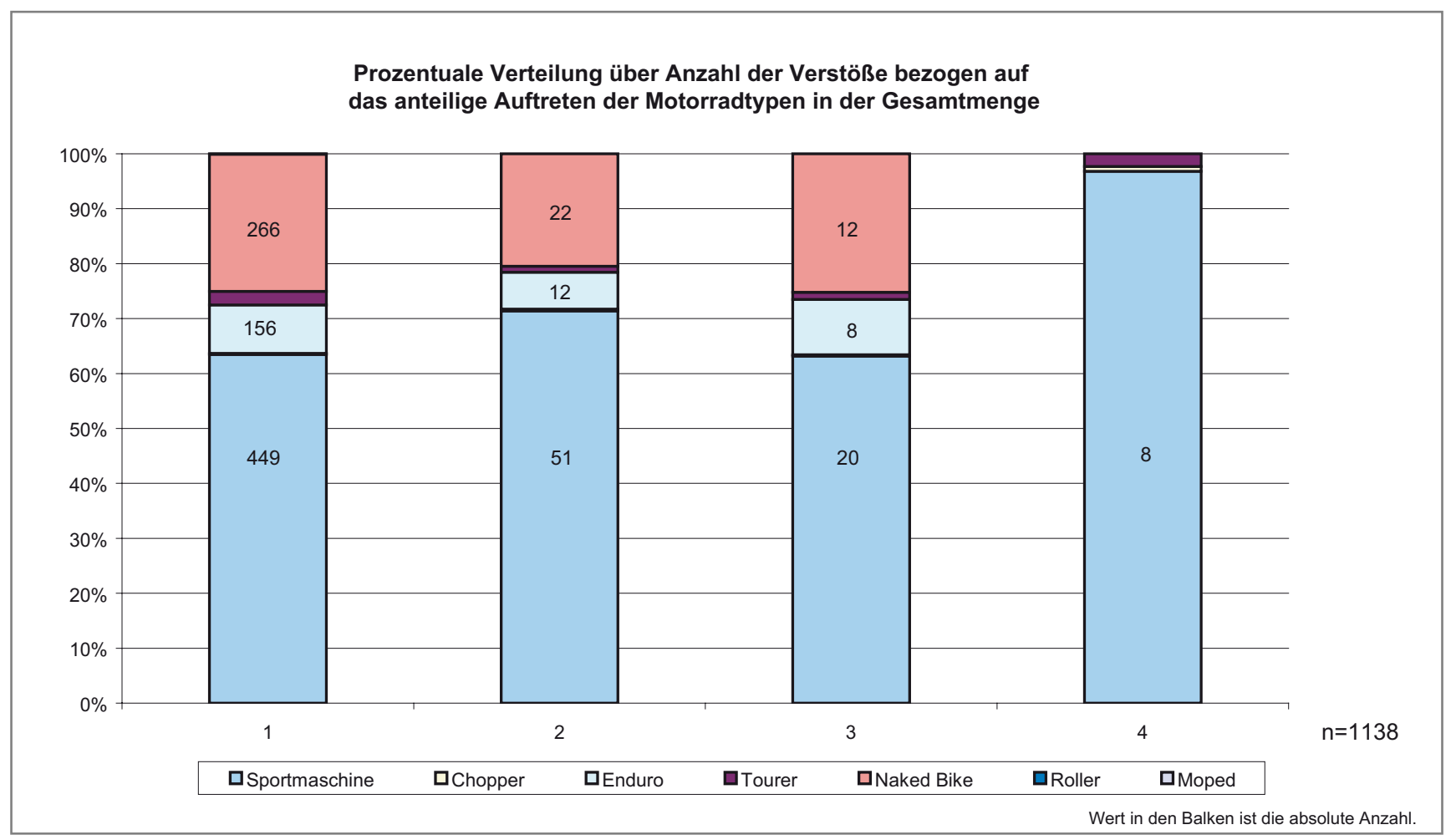

Abbildung 6-42: Verkehrsverstöße der Befragten über Motortradyp 


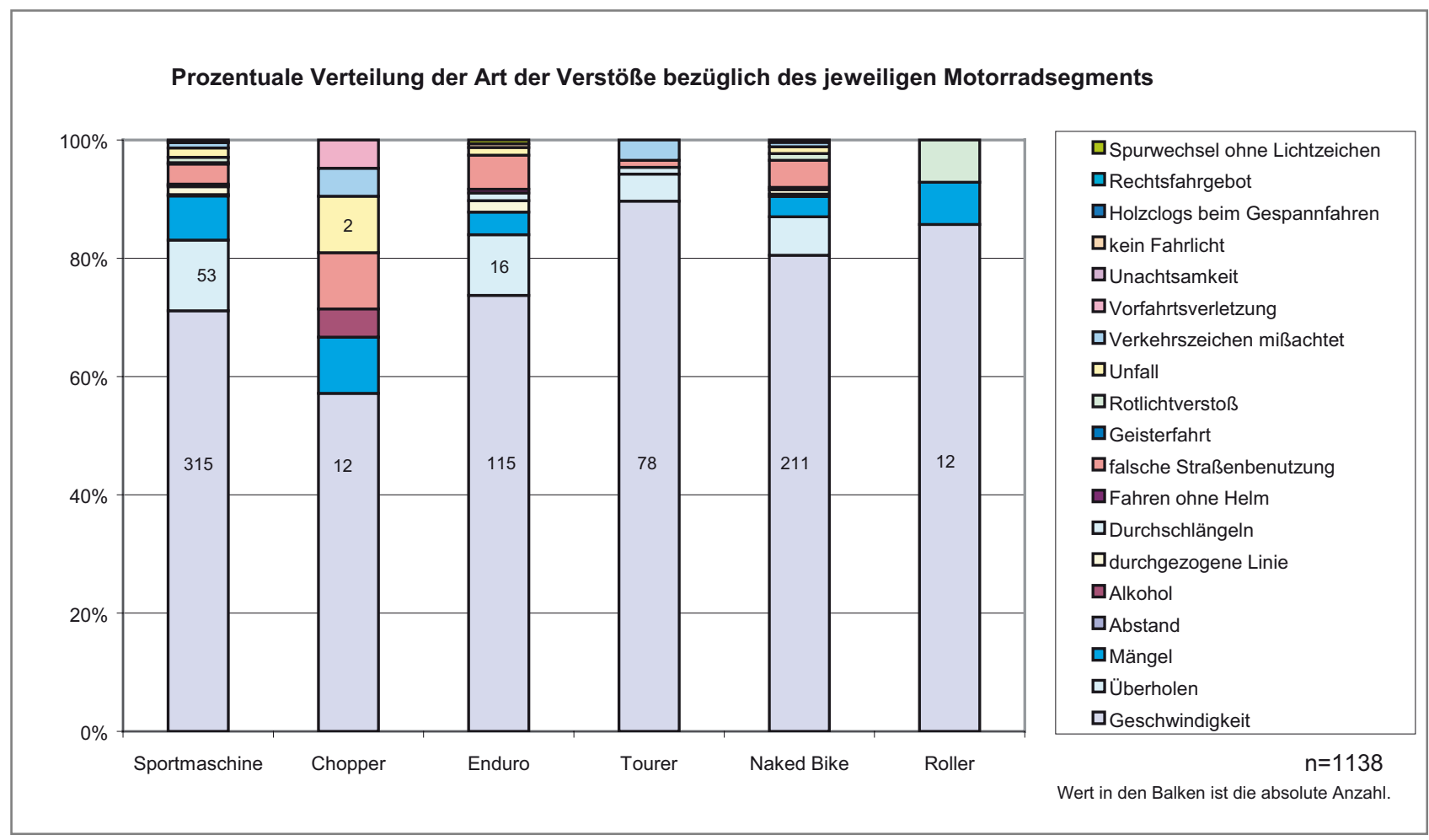

Abbildung 6-43: Art der Verkehrsverstöße

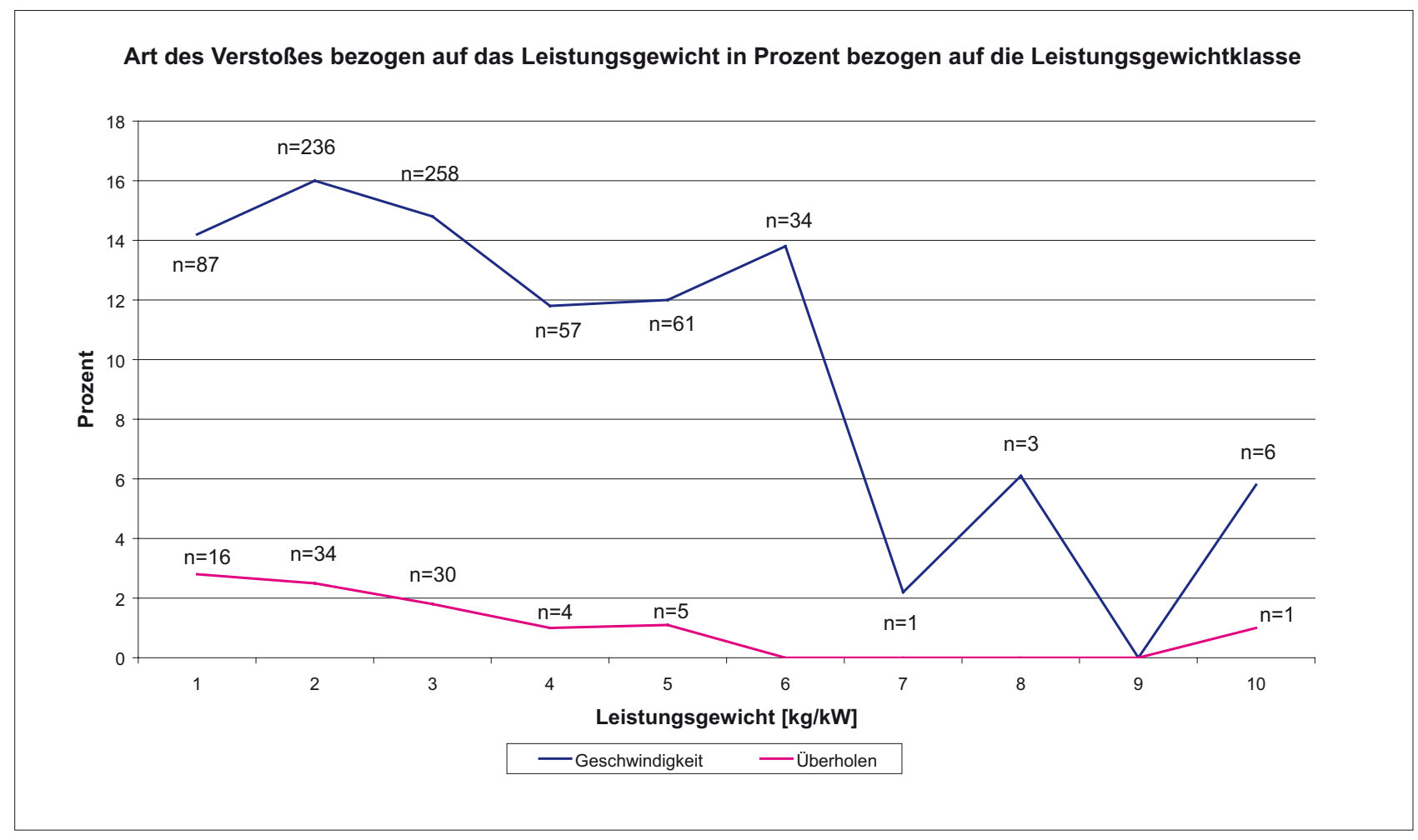

Abbildung 6-44: Art des Verstoßes über das Leistungsgewicht 


\subsubsection{Tragen von Sicherheitskleidung}

Da der Motorradfahrer nicht wie im Pkw durch eine Sicherheitszelle geschützt werden kann, ist er von Schutzbekleidung abhängig, deren Benutzung jedoch bei privaten Motorradfahrten vom Gesetzgeber nicht vorgeschrieben ist. Aus Bequemlichkeit wird bei kurzen Strecken in der Stadt gerne auf eine komplette Schutzbekleidung verzichtet, da es lästig ist diese ständig an und aus zu ziehen. Die Bereitschaft Schutzkleidung zu tragen, ist zudem stark vom Motorradsegment abhängig. Abbildung 6-45 zeigt für die verschiedenen Motorradsegmente das Trageverhalten von sicherer Bekleidung, die regelmäßig getragen wird. Lederkombinationen werden am meisten von Sportmaschinenfahrern getragen. Diese liegt dem Körper eng an und flattert nicht im Fahrtwind, wodurch Fahrwerksunruhen, eingeleitet durch den Fahrer, vermieden werden. Die bequemere Textilbekleidung in Form von Protektorenkombinationen wird von allen Fahrern bis auf Fahrer von Choppern und Rollern getragen.

Die Tragerate bei Handschuhen ist durch alle Segmente hindurch als sehr gut zu bezeichnen. Über 80 \% der Probanden tragen regelmäßig Handschuhe. Auch Motorradstiefel werden zu über 80 \% getragen; nur Rollerfahrer tragen sie zu ca. 50 \%, was durch die geschützte Sitzposition erklärt werden kann, die einen Sicherheitsvorsprung suggeriert, der in der Realität nicht gegeben ist.

Der Integralhelm wird von fast allen Fahrern außer Rollerund Chopperfahrern zu über $90 \%$ verwendet. Roller- und
Chopperfahrer tragen verstärkt offene Jethelme oder sogar nur nicht CE-geprüfte „Braincaps“, welche nur den obersten Teil des Kopfes abdecken und praktisch keine Schutzwirkung haben.

Zusammenfassend kann bemerkt werden, dass alle Motorradfahrer regelmäßig, bis auf Chopper- und Rollerfahrer, sichere Schutzkleidung tragen.

Als Gegendarstellung von Tragen sicherer Schutzbekleidung stellen die Abbildung 6-46 und Abbildung 6-47 die Tragefrequenz weniger geeigneter Bekleidung auf dem Motorrad dar.

Wieder wird erkennbar, dass Chopper- und Rollerfahrer weniger auf Sicherheitsbekleidung setzen. $40 \%$ der Chopperfahrer geben an, immer mit einem Jethelm Motorrad zu fahren. Nur 30 \% tragen nie einen Jethelm. Bei den Rollerfahrern ist das Verhältnis $20 \%$ zu 45 \%. Als Gegenbeispiel dienen die Sportmaschinenfahrer, die zu über $90 \%$ nie auf einen Integralhelm verzichten.

Ähnlich verhält es sich bei dem Tragen von Jeanshosen. Ihr fest gewebter Stoff täuscht einen guten Abriebwert vor, doch bietet eine Jeanshose beim Rutschen über den Asphalt kaum Schutz. Sie brennt sich durch die Hitzeentwicklung beim Abreiben tief in die Wunden ein. Nur $20 \%$ der Chopperfahrer tragen nie eine Jeans beim Motorradfahren, $10 \%$ dagegen immer. Bei den Rollerfahrern sind es ca. $12 \%$, die nie eine Jeans tragen.

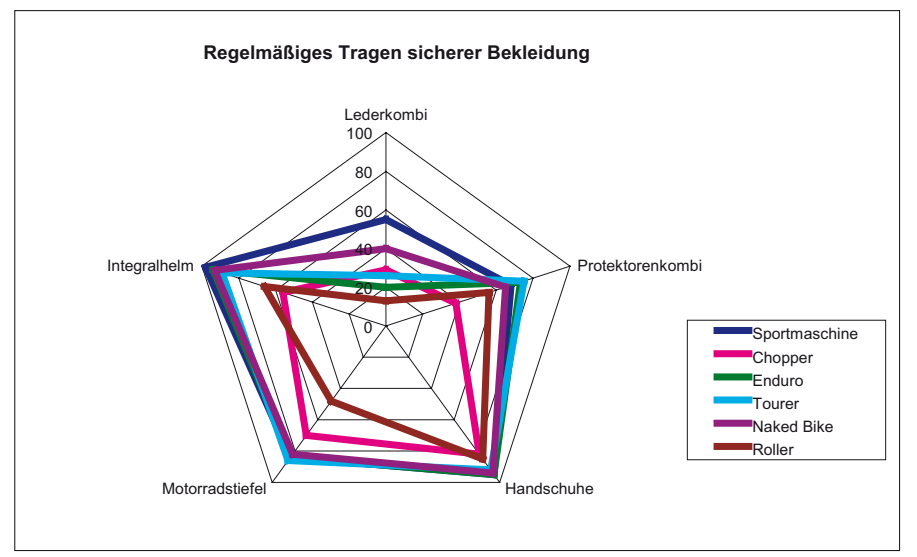

Abbildung 6-45: Trageverhalten sicherer Schutzkleidung beim Motorradfahren 


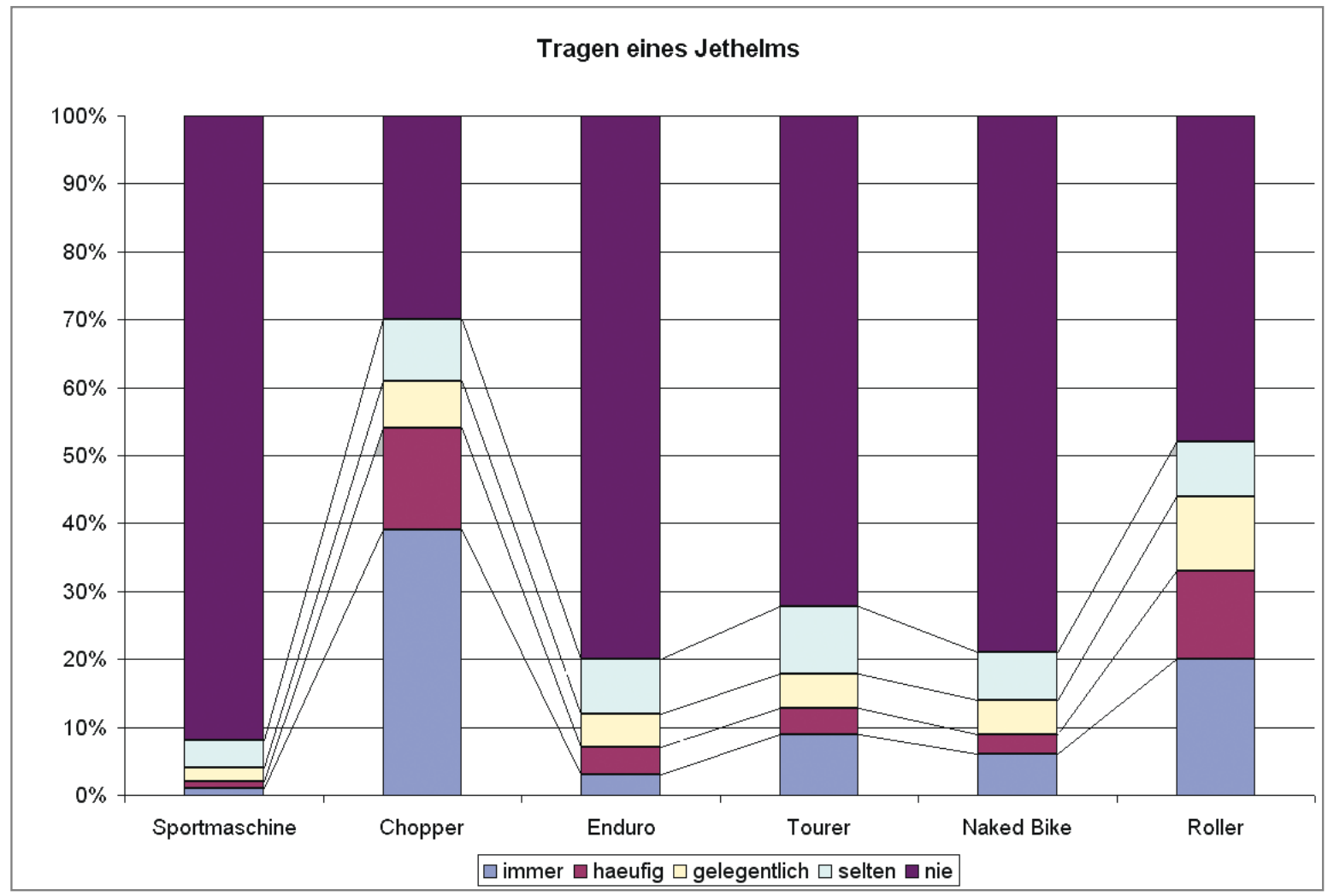

Abbildung 6-46: Trageverhalten eines Jethelmes

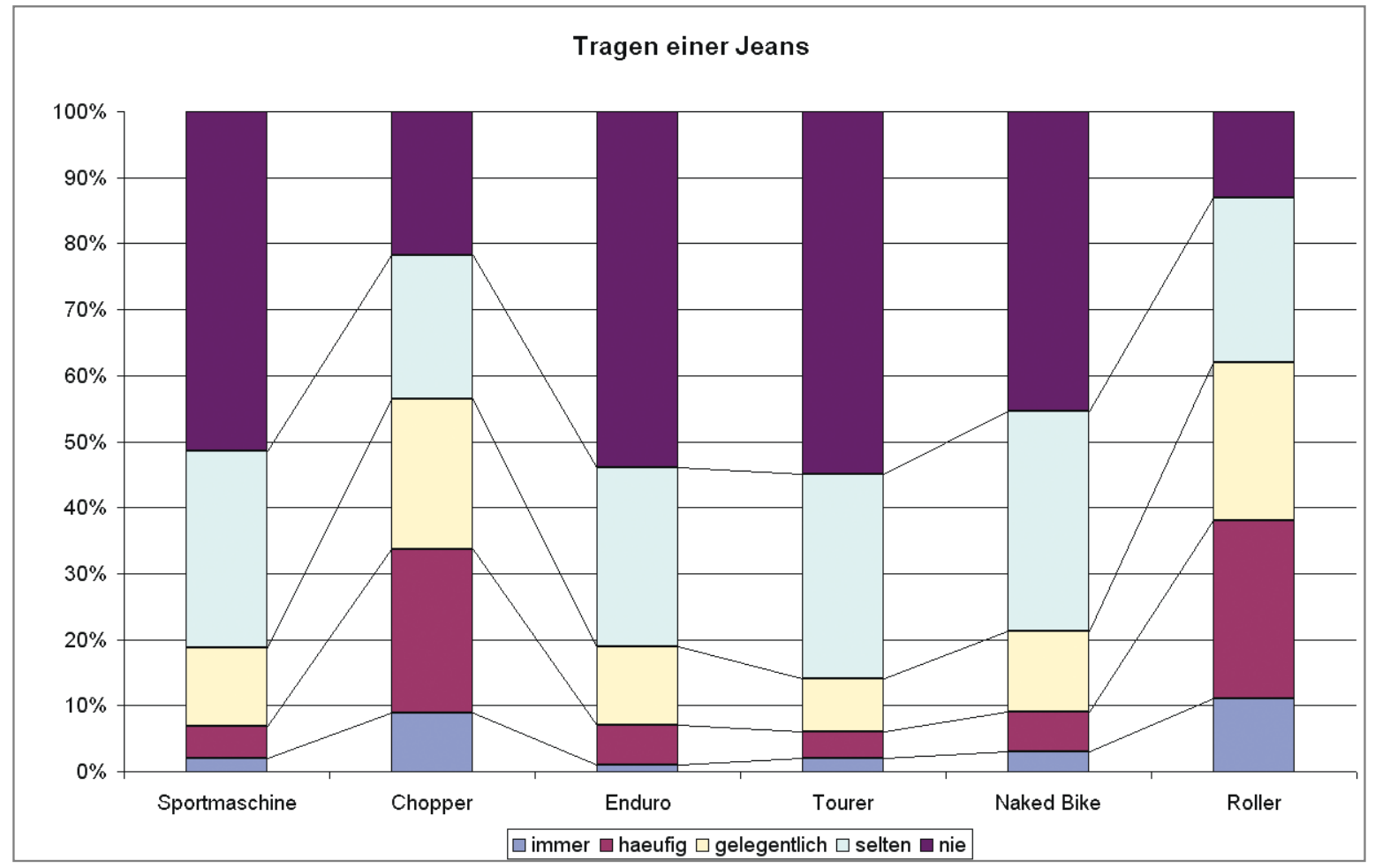

Abbildung 6-47: Trageverhalten einer Jeans 


\subsubsection{Unfallbetrachtung}

Getrennt nach selbst- und fremdverschuldeten Unfällen (Abbildung 6-48 und Abbildung 6-49) ist zu erkennen, dass keine besonderen Auffälligkeiten für einen einzelnen Motorradtypen beobachtet werden können. Unfälle innerhalb geschlossener Ortschaften sind zu zwei Dritteln nicht durch eigenes Handeln verschuldet. Es sind jedoch deutliche Unterschiede in der Aufteilung der selbst- zu den fremdverschuldeten Unfällen bei den Motorradtypen zu erkennen: Der Anteil der Selbstverschuldeten Unfälle auf Landstraßen ist bei Sportmaschinen und Naked Bikes beispielsweise deutlich höher als bei Tourerfahrern.

Ist der Motorradfahrer der Hauptschuldige, so ereignen sich zwei Drittel der betreffenden Unfälle außerhalb geschlossener Ortschaften. Abbildung 6-50 zeigt, dass zudem die meisten selbstverschuldeten Unfälle ohne andere Beteiligung sind, also Alleinunfälle. Auch hier gibt es die ein-zu-zwei-Drittel Regel. Über die verschiedenen Motorradtypen ist keine Auffälligkeit auszumachen. Nur bei den Rollerfahrern gibt es Abweichungen, die wegen der geringen Anzahl jedoch als statistisch irrelevant eingestuft werden sollten.

Für die Verteilung der Alleinunfälle bezogen auf Leistungsgewicht und Ortslage (Abbildung 6-51) ist zu erwähnen, dass sich mit steigendem Leistungsgewicht prozentual mehr Alleinunfälle in geschlossenen Ortschaften ereignen. Fahrer leichter und leistungsstarker Motorräder erleiden außerhalb geschlossener Ortschaften häufiger einen Alleinunfall als Fahrer schwerer und leistungsschwächerer Maschinen.

Ein Zusammenhang mit der Verletzungsschwere kann nicht dargestellt werden, da die meisten berichteten Unfälle ohne oder mit leichten Verletzungen geschehen sind. Nur ein Bruchteil der Fälle beinhaltet schwer verletzte Personen. Getötete Motorradfahrer sind in der Datenmenge nicht enthalten. Eine statistische Auswertung bezogen auf die Verletzungsschwere führte zu keinen relevanten Aussagen.

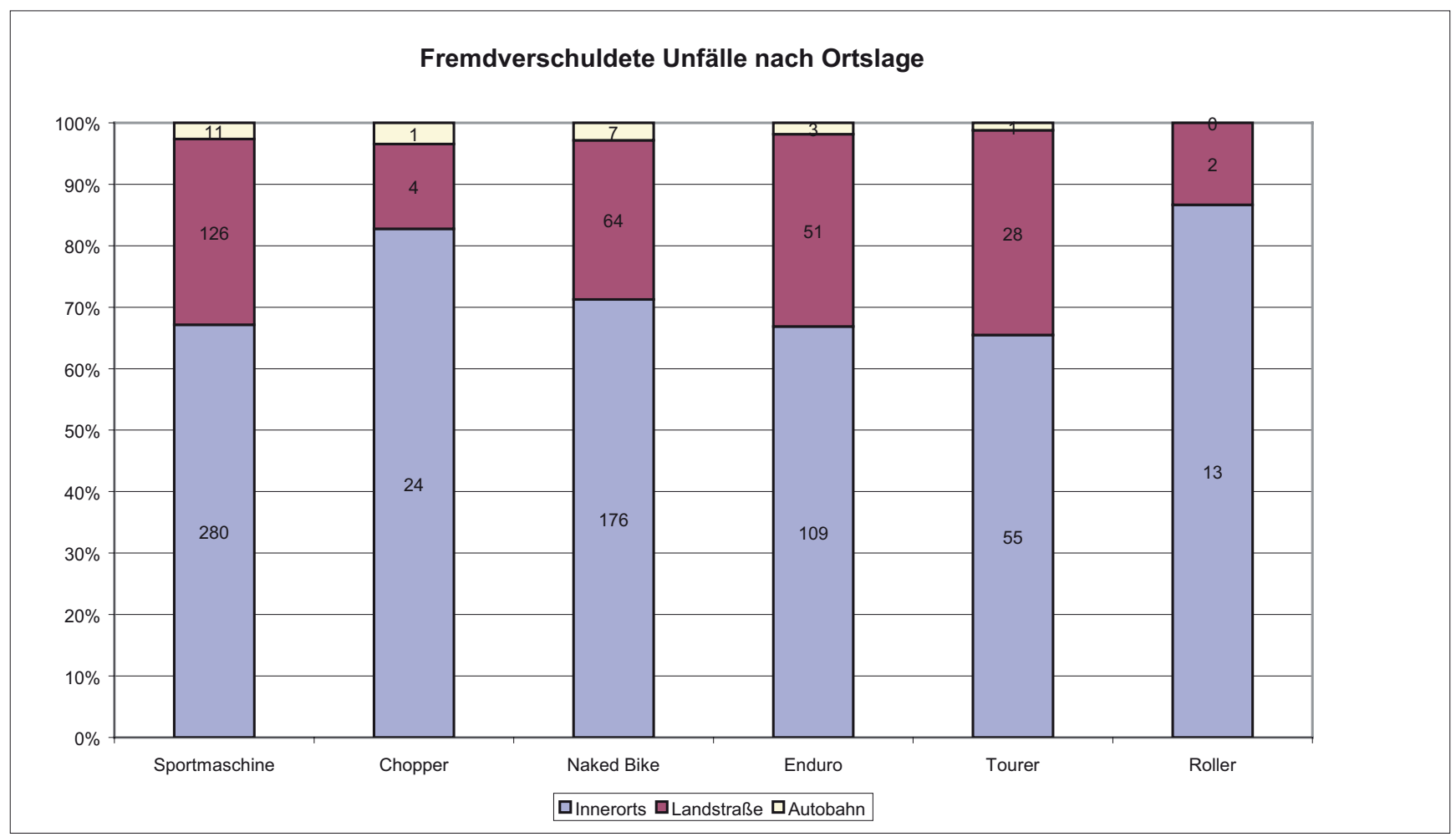

Abbildung 6-48: Verteilung der fremdverschuldeten Unfälle nach Ortslage und Motorradtyp 


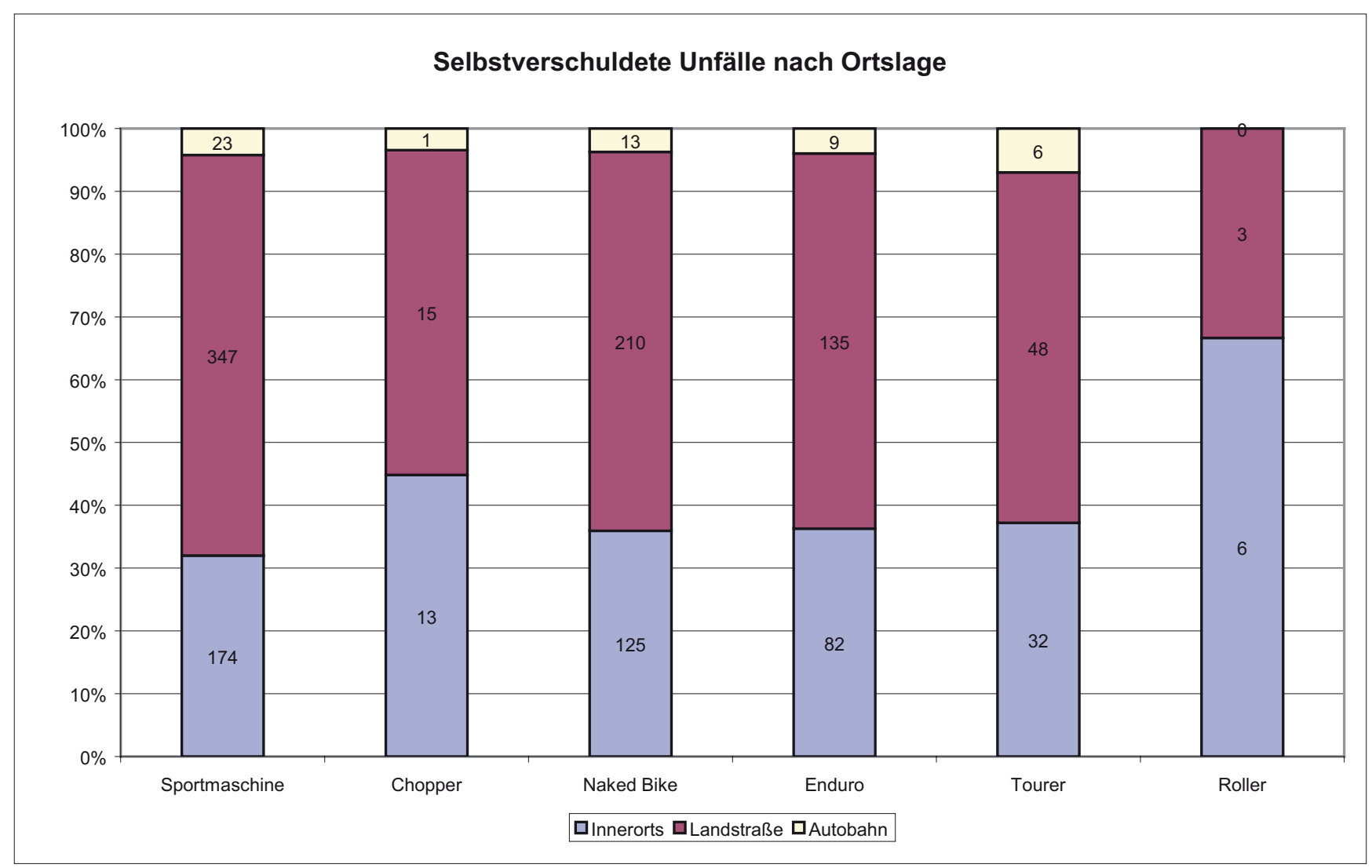

Abbildung 6-49: Verteilung der verschuldeten Unfälle nach Ortslage und Motorradtyp

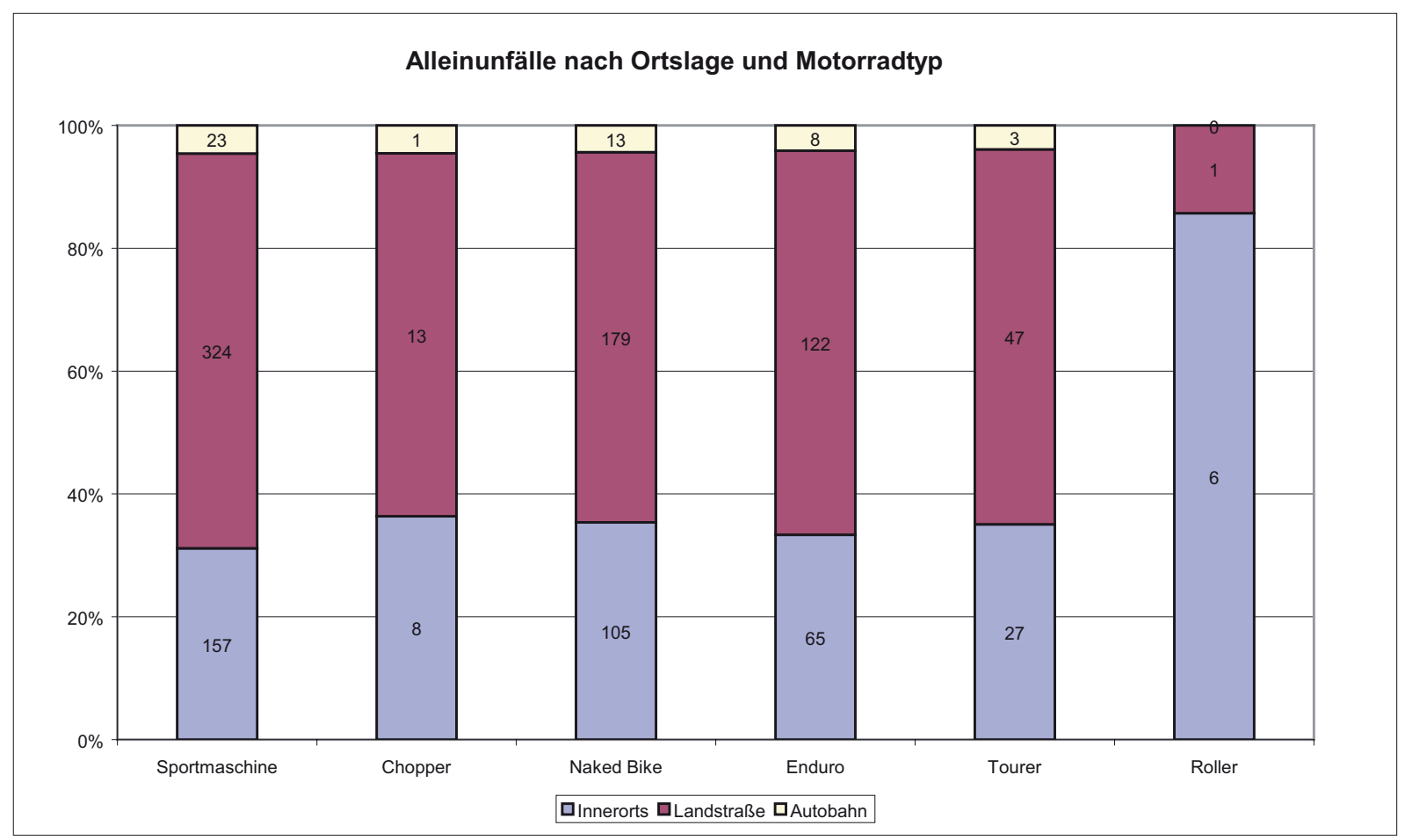

Abbildung 6-50: Alleinunfälle nach Ortslage und Motorradtyp 


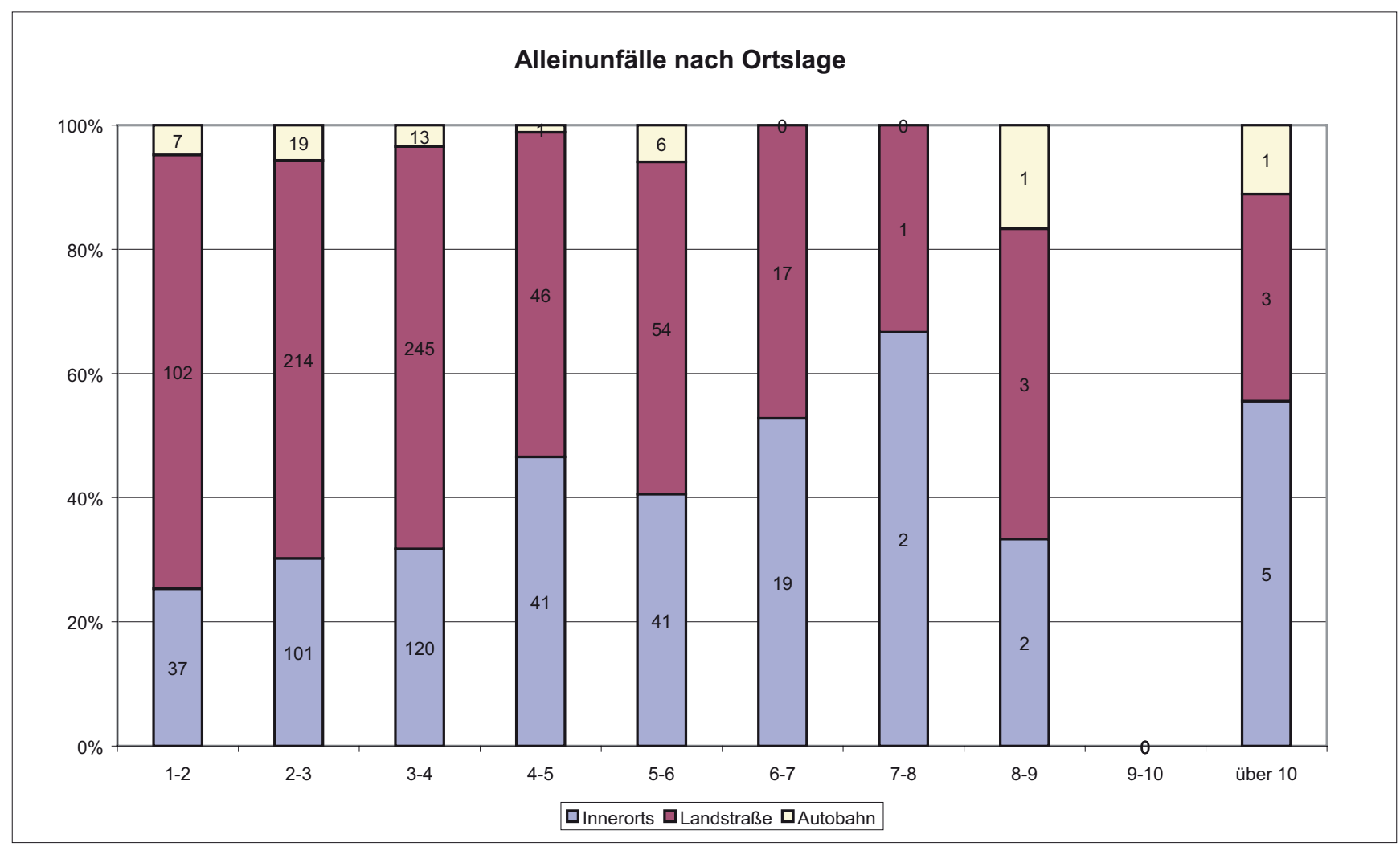

Abbildung 6-51: Alleinunfälle nach Leistungsgewicht und Ortslage

Darüber hinaus können noch die folgenden unfallbezogenen Erkenntnisse aus den Befragungsdaten abgeleitet werden:

- Zwischen den Unfalltypen gibt es keine signifikanten Unterschiede im Alter bei Unfall, gefahrene Jahreskilometern, Leistungsgewicht und Anzahl der Verkehrsverstösse.

- Unfallfahrer schätzen ihr Fahrkönnen höher ein als unfallfreie Fahrer.

- Unfallfahrer beschreiben ihren Fahrstil eher als sportlicher (weniger bedacht) als unfallfreie Fahrer.

- Unfallfahrer haben in den letzten drei Jahren signifikant mehr Verkehrsregelverstösse begangen.

- Die Chance, mit einem Motorrad mit ABS zu verunglücken, ist deutlich geringer als Chance, mit einem Motorrad ohne ABS einen Unfall zu haben.

- Die Chance, mit einem modifizierten Motorrad zu verunglücken, ist größer als mit einem serienmäßigen Motorrad. Dies gilt vor allem bei modifizierten Sportmaschinen, aber auch bei modifizierten Enduros.
- Sportmaschinenfahrer haben mehr Verkehrsverstösse begangen als Fahrer von Enduros und Fahrer von Naked Bikes.

\subsubsection{Zusammenfassung}

Die Daten der Motorradumfrage zeigen eindrücklich, dass im Gegensatz zu den von der Polizei aufgenommenen Unfällen, der Grund des Unfalls vom Motorradfahrer bei sich selbst gesehen wird. Viele Probanden antworteten aufgrund der Anonymität mit der Einschätzung, dass sie selbst an einem Unfall schuld seien. Es wurden selten Fahrbahnzustände oder andere, nicht beeinflussbare Faktoren „vorgeschoben“. Hier spielt sicher auch die „Dunkelziffer" eine Rolle, da gerade Alleinunfälle mit höherer Selbstverursachung in einer Befragung häufiger bekannt werden als bei der polizeilichen Unfallaufnahme. Außerdem wird ein Mitverschulden beim Unfallbeteiligten anders interpretiert als die Festlegung des Hauptunfallverursachers nach polizeilicher Einschätzung vor Ort. 
Das Hauptergebnis der Befragung ist in der Beschreibung der Motorradfahrergesinnung zu sehen. Dabei fallen besonders Sportmaschinenfahrer auf, die besonders häufig in Ordnungswidrigkeitsdelikte verwickelt sind, wie z. B. zu hohe Geschwindigkeit oder falsche Überholmanöver. Sie geben selbst an, dass sie sich als sportliche Fahrer verstehen. Das lässt darauf schließen, dass sie an dieser Stelle gerade den „Thrill“, also die Fortbewegung an einem weniger kalkulierbaren Risikolevel, suchen. Bei der Motivation geben sie zu $50 \%$ an, dass sie die Sportlichkeit, das Beschleunigungsvermögen und die Geschwindigkeit am Motorradfahren schätzen. Der Zusammenhang zu den Verstößen ist damit gegeben.

Auch der Zusammenhang mit den Ergebnissen der GDVDatenanalyse ist gegeben. Sportmotorräder mit einem besonders niedrigen Leistungsgewicht, bei denen das Beschleunigungsvermögen hoch ist, sind überproportional schwer in Unfälle verwickelt. Hier zeigt sich, dass die Gefahr eines Unfalls als "Nervenkitzel“ in Kauf genommen wird, geradezu gesucht wird. 


\section{Zusammenführung der Ergebnisse}

Gemäß dem Projektverlauf wurden von der TU Berlin insbesondere fahrzeugtechnische Eigenschaften der motorisierten Zweiräder sowie personenbezogene Daten des Aufsassen untersucht, während von der TU Dresden vor allem die straßenseitigen Eigenschaften im Zusammenhang mit dem Unfallgeschehen von motorisierten Zweirädern betrachtet wurden. Um interdisziplinär gemeinsame Lösungsansätze zum Senken der Verunglücktenzahlen von Fahrern motorisierter Zweiräder aufzustellen, ist es notwendig, dass die Ergebnisse der Unfallanalysen trotz unterschiedlicher Datenbasen verknüpfbar gemacht werden.

Ausgehend von Abbildung 5-5 stellt die Bildung typischer Unfallkonstellationen auf Basis von Ortslage, Charakteristik der Unfallstelle, Unfalltyp und Unfallart die Verbindung zwischen den Datenbasen dar (vgl. hierzu Abschnitte 5.1.4, 5.2.4 sowie insbesondere Abbildung 5-18). Abbildung 7-1 stellt die Fallzahlen der fahrzeugtechnischen und straßenseitigen Betrachtung für die zehn Unfallkonstellationen gegenüber.

Die Konstellationen 2 sowie 4-6 sind in den GDV-Daten deutlich stärker vertreten. Dies ist in der größeren Datenbasis (1304 Unfälle gegenüber 530 Unfällen auf den besichtigten Streckenabschnitten) begründet. Die Unfallkonstellationen 1, 3 und 7-10 treten seltener, gleich häufig oder gar nicht in den GDV-Daten auf. Dies kann für die Konstellationen 7, 8 und 10 damit erklärt werden, dass diese die geringste Unfallschwere aufweisen. Die GDV-Daten beinhalten ausschließlich Motorradunfälle über einem Gesamtschaden von $15.000 €$ und nur Unfäl-

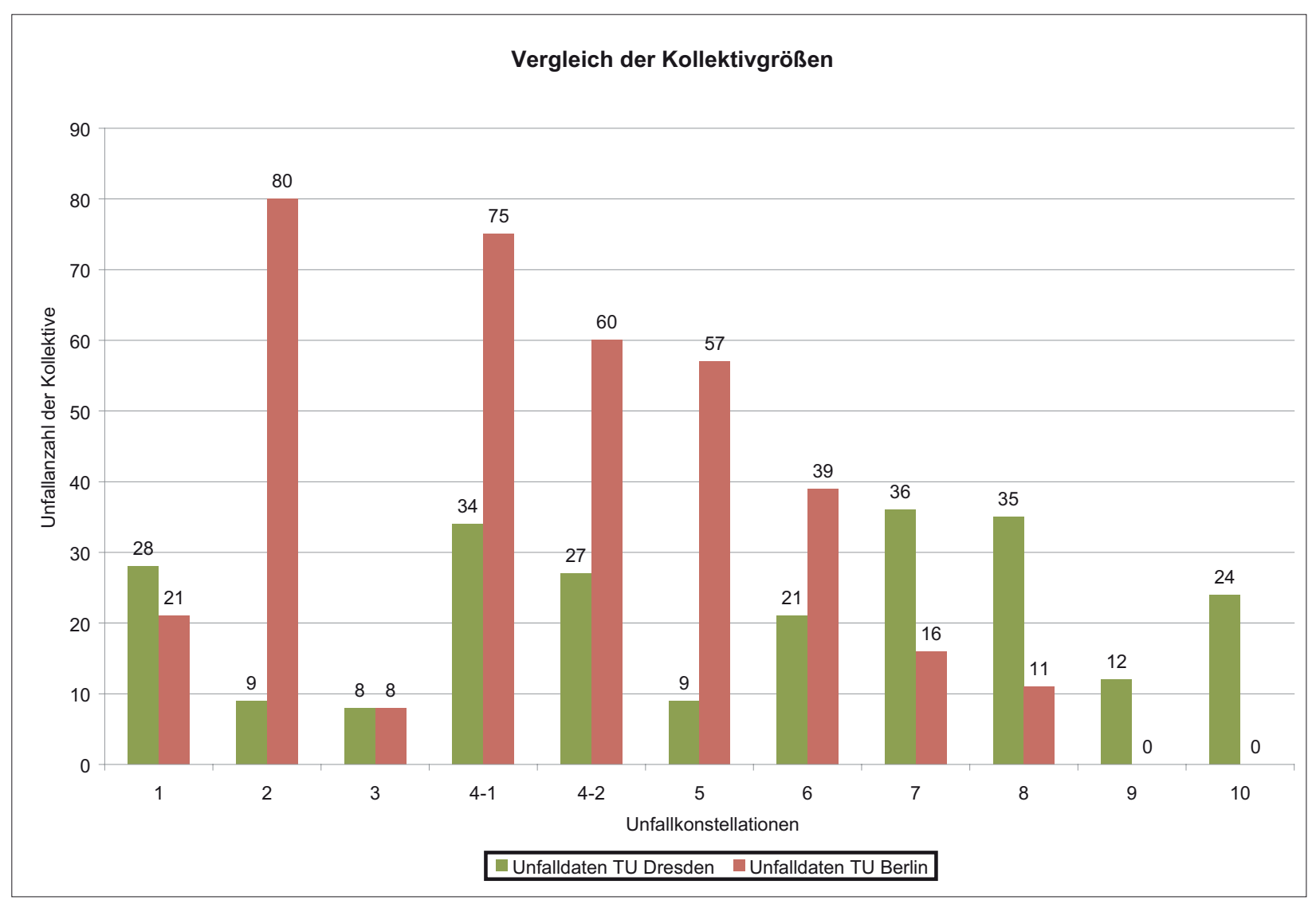

Abbildung 7-1: Vergleich der Kollektivgrößen von TU Berlin und TU Dresden für die Unfallkonstellationen 
le, die der Versicherung gemeldet wurden. Somit werden Alleinunfälle ohne Haftpflichtschaden bei Motorrädern ohne Vollkaskoversicherung nicht erfasst. Dies erklärt wiederum die geringen Zahlen für die Konstellationen 1 und 3 (Fahrunfälle in Kurven). Auf Grund dieser unterschiedlichen Beschaffenheit der beiden Datenbasen weisen die Kollektive der Konstellationen auch deutliche Ungleichheiten in der Unfallschwere auf. Die Unfälle aus den GDV-Daten fallen für fast jede Unfallkonstellation im Mittel schwerer aus, da nicht alle Sachschadensunfälle enthalten sind.

Von Seiten der TU Berlin wurden für die statistisch begründeten Auswertungen ausschließlich die Unfallkonstellationen ausgewertet, die mehr als 30 Unfälle beinhalten. Dabei handelt es sich um die Konstellationen 2 sowie 4 bis 6 . Bei den übrigen Konstellationen beschränkt sich der Blick somit auf das Unfallgeschehen und die straBenseitigen Eigenschaften. Die Ergebnisse zu den Unfallkonstellationen werden im Folgenden dargestellt, die detaillierten Resultate beinhaltet Anlage 08.

\subsection{Unfallkonstellation (1)}

Insgesamt treten in der Datenbasis der TU Dresden 48 Fahrunfälle in Kurven außerorts auf. Die hauptsächliche Unfallart ist dabei das Abkommen von der Fahrbahn nach rechts (Unfallart 8, Beschreibung der Unfallarten siehe Anlage 10) mit 29 Unfällen. Weitere sieben Fahrunfälle in Kurven sind mit dem Abkommen von der Fahrbahn nach links verbunden, bei weiteren acht Fahrunfällen kam es in der Kurve zu einem Zusammenstoß mit einem entgegenkommenden Fahrzeug. Diese Fälle wurden nicht weiter untersucht. Die 28 Unfälle dieser Konstellation werden ausschließlich aus Fahrunfällen ohne Beteiligung weiterer Verkehrsteilnehmer, die mit einem Abkommen von der Fahrbahn nach rechts sowie einem Personenschaden verbunden sind, gebildet.

Aus dem Abkommen in einer Kurve nach rechts kann aufgrund der fahrdynamischen Gesetzmäßigkeiten (Radialkraft, die quadratisch von der Fahrgeschwindigkeit abhängt, wirkt in Richtung Kurvenaußenseite) in der Regel auf eine Linkskurve geschlossen werden. Eine dazu stichprobenhaft durchgeführte Untersuchung (50 Fahrunfälle mit Unfallart 8 auf Bundes- oder Staatsstraßen in Sachsen) ergab, dass $93 \%$ der auswertbaren Unfälle nach VUA sich tatsächlich in einer Linkskurve ereigneten. Fahrunfälle in Kurven treten demnach deutlich öfter in Links- als in Rechtskurven auf. In den Empfehlungen der europäischen Motorradhersteller für eine sichere Straßengestaltung im Blick auf die Motorradfahrer [ACEM 2006] wird das Befahren von Linkskurven für einen PTW-Fahrer im Allgemeinen als schwieriger eingeschätzt als das Befahren von Rechts-

\begin{tabular}{|l|l|}
\hline Alleinunfall in Außerortskurve mit Abkommen von der Fahrbahn nach rechts (i. d. R. Linkskurve) \\
\hline Unfallkollektiv & 28 \\
\hline Unfallschwere: Anteil U (SP) & $68 \%$ \\
\hline Anteil Hauptverursacher PTW & $100 \%$ \\
\hline Anteil Unfälle am Wochenende & $50 \%$ \\
\hline Auffälligkeiten Unfallauswertung & - über 2/3 der Unfälle mit Aufprall auf ein Hindernis neben der \\
& Fahrbahn \\
& - häufigstes Hindernis dabei: die Schutzplanke \\
& - Unfallzeiten deuten vor allem Freizeitverkehr hin \\
& - kein negativer Einfluss durch \\
& nasse Fahrbahn oder Dunkelheit \\
\hline Auffälligkeiten Straßenraum: & - mit einer Kurvigkeit > 225 gon/km (39\% vs. 22\%) \\
Überdurchschnittlicher Anteil & - mit einer Fahrbahnbreite unter 6 m, ohne Mittelmarkierung \\
von Unfällen auf Abschnitten & und gleichzeitig einer Kurvigkeit > 225 gon/km (21\% vs. 11\%) \\
\hline
\end{tabular}

Tabelle 7-1: Übersicht zur Unfallkonstellation (1) 
kurven: Während der Durchfahrt einer Rechtskurve fixiert der PTW-Fahrer die Kurveninnenseite. Da der Kurs des PTW ebenfalls entlang der Innenseite verläuft, kann die vor ihm liegende Strecke mit demselben Blickwinkel überschaut werden. Bei der Durchfahrt einer Linkskurve fixiert der PTW-Fahrer auch die Kurveninnenseite, der Kurs des PTW verläuft aber entlang der Kurvenaußenseite. Somit muss der PTW-Fahrer ständig den Blickwinkel zwischen der Kurveninnenseite, die ihm als Orientierungspunkt dient, und der unmittelbar vor ihm liegenden Strecke, auf der bspw. Hindernisse erscheinen können, wechseln.

Kennzeichnend für die Unfallkonstellation sind eine hohe Unfallschwere und mit 50 \% ein hoher Anteil von Unfällen, die am Wochenende (Sa/So) passieren. Dies zeigt ebenso wie ein leichter Anstieg der Unfälle in den Sommermonaten bzw. in der Ferienzeit, dass es sich besonders um Ausflugs- und Freizeitverkehr handelt. In den Wintermonaten November bis März finden dagegen allein zwei Unfälle statt. Die Merkmale schlechter Straßenzustand (nass/winterglatt) und ungünstige Lichtverhältnisse können mit einem Anteil von jeweils $4 \%$ als unfallbegünstigend ausgeschlossen werden. Die hohe Unfallschwere hängt neben den außerorts zu vermutenden hohen Geschwindigkeiten mit einem Aufprall auf ein Hindernis neben der Fahrbahn zusammen. In reichlich 2/3 der Fälle erfolgte ein solcher Aufprall, bei etwa der Hälfte davon an eine Schutzplanke. Sonstige Hindernisse stellen das zweitgrößte Problem bei einem Aufprall dar (6 Unfälle). Der Blick auf die Zielgruppen zeigt für keine Gruppe eine Auffälligkeit.

Die Auswertung der Anteile von Unfällen, die sich auf einem Streckenabschnitt mit einer bestimmten Ausprägung einer Straßeneigenschaft ereignet haben, bringt hohe Anteile auf Abschnitten, die eines der folgenden Kriterien erfüllen:

- Kurvigkeit von mehr als 225 gon/km (39\%) Steigung/Gefälle nach Z 108/110 StVO (32 \%)

- mindestens eine Kuppe in unmittelbarer Nähe einer Kurve oder eines Knotens (46\%)

- mindestens 1/4 der Strecke führt durch Waldgebiet oder entlang regelmäßiger Hindernisse im Seitenraum (54\%).
Der Vergleich mit den Unfallanteilen aller Außerortsunfälle auf den besichtigten Streckenabschnitten, zeigt die erkannten Auffälligkeiten in Tabelle 7-1.

Die bestehenden Zusammenhänge mit Trassierungsparametern wurden für diese Unfallsituation bereits ausführlich in [Gerlach 2007] untersucht. Daher wurden vertiefende Auswertungen wie bspw. die genaue Zuordnung der Unfälle zu Kurvenbereichen mit bestimmten Radien, Radienfolgen, Richtungsänderungen etc. nicht durchgeführt. Die Untersuchung in [Gerlach 2007] konnte bei ihren Auswertungen sowohl auf einer höhere Unfallanzahl als auch eine digitale Straßendatenbank von RheinlandPfalz mit einer Vielzahl von Trassierungsparametern zurückgreifen, so dass mit dem vorliegenden Datenmaterial keine Verbesserung der bereits gewonnenen Erkenntnisse erwartet werden kann. Wichtige Zusammenhänge wurden diesbezüglich in Abschnitt 3.1.2 dargestellt.

\subsection{Unfallkonstellation (2):}

Die Unfallkonstellation setzt sich aus 9 bzw. 80 Einbiegen/Kreuzen-Unfällen an Einmündungen zusammen, die der Unfallart 5 - Zusammenstoß mit einem Fahrzeug, das einbiegt oder kreuzt - zugewiesen waren. Die Erkenntnisse aus Sicht der Verkehrstechnik werden wie folgt zusammengefasst, die geringe Kollektivgröße ist bei den Aussagen zu berücksichtigen:

\section{Unfallgeschehen und Straßeneigenschaften}

Über die Hälfte der neun Unfälle sind Unfälle mit schwerem Personenschaden, knapp 90 \% Unfälle mit Personenschaden. Dabei sind schlechte Lichtverhältnisse als unfallbegünstigender Faktor nicht auszuschließen. Der Anteil liegt mit 22 \% über dem Wert bei Auswertung der Unfälle aller definierten Außerortsabschnitte in Sachsen (14\%). Vier der Unfälle ereigneten sich nach VUA an Knoten mit LSA, in drei Fällen war diese auch zum Unfallzeitpunkt in Betrieb. Hierbei wird davon ausgegangen, dass es sich um Rotüberfahrten handelte. Durch die Auswertung der Unfalltexte konnte dies in einem Fall bestätigt werden, für die anderen beiden Unfälle standen keine Texte zur Verfügung. 


\begin{tabular}{|l|l|}
\hline $\begin{array}{l}\text { Einbiegen/Kreuzen-Unfall } \\
\text { außerorts an einer } \\
\text { Einmündung }\end{array}$ \\
\hline Unfallkollektiv & 9 (TU Dresden / 80 (TU Berlin \\
\hline Unfallschwere: Anteil U (SP) & $55 \%$ (TU Dresden / $84 \%$ (TU Berlin) \\
\hline Anteil Hauptverursacher PTW & $22 \%$ (TU Dresden) / $9 \%$ (TU Berlin) \\
\hline Anteil Unfälle am Wochenende & $33 \% /$ (TU Dresden) / $29 \%$ (TU Berlin) \\
\hline Auffälligkeiten Unfallauswertung & $1 / 3$ der Unfälle an LSA in Betrieb (Rotüberfahrten) \\
\hline Auffälligkeiten Straßenraum & $\begin{array}{l}\text { - mit einer Knotenpunktdichte > } 2 \text { Knoten/km (33 \% vs. 22 \%) } \\
\text { - mit Sichteinschränkung (22 \% vs. 17\%) }\end{array}$ \\
\hline Auffälligkeiten Fahrzeugtechnik & $\begin{array}{l}\text { - Sportmaschinenfahrer sind überrepräsentiert } \\
\text { - höhere Verletzungsschwere bei geringerem Leistungsgewicht } \\
\text { - frontaler Hauptanstoß in die Fahrerseitte des Unfallgegners } \\
\text { mit hoher, mittlerer Verletzungsschwere von MAIS 30,5 }\end{array}$ \\
\hline
\end{tabular}

Tabelle 7-2: Übersicht zur Unfallkonstellation (2)

Hauptverursacher war in zwei Fällen der Fahrer des PTW. Der Unfallgegner war in sieben Fällen ein Pkw, in einem Fall ein Lkw. Dies deutet daraufhin, dass bei einem Großteil der Fälle das PTW auf der vorfahrtberechtigten Straße verkehrt und bspw. aufgrund von Fehleinschätzung der Geschwindigkeit oder zu schlechter Sichtverhältnisse zu spät wahrgenommen wird. Die Untersuchung der Unfalltexte bestätigte dies.

Für sechs Unfälle lagen Unfalltexte vor. In fünf Fällen kam der Pkw als Unfallbeteiligter 01 aus der untergeordneten Zufahrt (einschließlich eine Rotüberfahrt), in vier Fällen wollte er dabei links in die übergeordnete Straße einbiegen. In dem verbleibenden Fall bog der Pkw nach rechts ein und kollidierte dabei mit einem PTW, welches sich gerade in einem Überholvorgang befand. In den erstgenannten vier Fällen näherte sich das PTW in drei Fällen aus Sicht des einbiegenden Fahrzeuges von links. Demnach ergibt sich die Abbildung 7-2 dargestellte typische Unfallsituation.

Bei der Untersuchung der Zielgruppen sind Auffälligkeiten in Bezug auf den mittleren Wert für alle Außerortsunfälle der definierten Streckenabschnitte in Sachsen für Frauen und Senioren zu erkennen, die aufgrund der kleinen Fallzahl aber auch zufällig sein können.
Der erste Blick auf die Auswertung bestimmter Straßeneigenschaften in Zusammenhang mit dem Unfallgeschehen unterstützt die These, dass Kuppen in Knotenpunktnähe in Form einer Sichtbehinderung als unfallbegünstigende Faktoren wirken können. 1/3 der Unfälle befindet sich auf Abschnitten, auf denen solche Hochpunkte auftreten. Bei genauerer Untersuchung stellt sich jedoch heraus, dass die drei Unfälle auf Abschnitten mit mindestens einem Hochpunkt vor einem Knoten alle auf demselben Abschnitt liegen. Ein Abgleich der Streckenskizze mit der Steckkarte zeigt, dass die Unfallposition allein für einen der drei Unfälle mit dem Knoten in Verbindung zu bringen ist, vor welchem die Kuppe liegt. Zumindest in dem Fall kann aber tatsächlich ein Einfluss angenommen werden, da der getötete Fahrer des PTW, welcher mit einem linkseinbiegenden Pkw kollidierte, bei Tageslicht aus der Richtung auf der übergeordneten Straße heranfuhr, in welcher sich der Hochpunkt befindet.

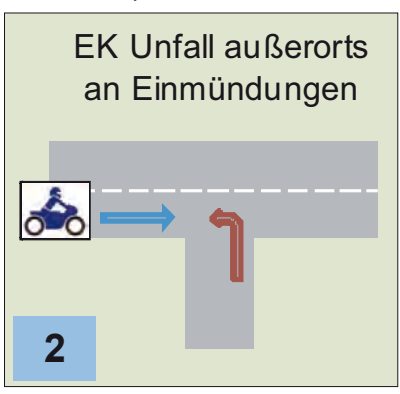

Abbildung 7-2: Typische Unfallsituation zur Unfallkonstellation (2) 


\section{Unfallgeschehen und Fahrzeugtechnik}

EK-Unfälle außerorts an Einmündungen stellen innerhalb der vier Konstellationen, die anhand der GDV-Daten untersucht wurden, die Unfallkonstellation mit der höchsten Unfallschwere dar. In den meisten Fällen war der PTW-Fahrer vorfahrtberechtigt und damit nicht der Hauptverursacher. In diesen Fällen fährt ihm entweder der Unfallgegner in seine Bahn und trifft ihn an der rechten Seite oder der PTW-Fahrer fährt frontal in die Seite des Unfallgegners. Die Verletzungsschwere liegt bei einem frontalen Auftreffen des Motorradfahrers auf den Unfallgegner im Schnitt um einen MAIS-Wert höher. Stürzt der Motorradfahrer vor dem Eintreten der Kollision führt dies im Mittel zu einer höheren Unfallschwere, als wenn er in aufrechter Fahrt frontal in die Seite des Unfallgegners fährt. Dies ist dadurch begründet, dass bei einem Sturz i. d. R. weniger kinetische Energie vor der Kollision abgebaut werden kann als im Fall einer durchgeführten Gefahrenbremsung bis unmittelbar vor der Kollision.

Von neun Unfällen unter dem Einfluss von Alkohol fallen fünf in diese Konstellation. Unter dem Einfluss von Alkohol oder einer überhöhten Geschwindigkeit sind bei den Unfällen im Mittel höhere Verletzungsschweren festzustellen. Zu $80 \%$ ist hierbei der PTW-Fahrer vorfahrtberechtigt und damit nicht der Hauptverursacher des Unfalles.

\subsection{Unfallkonstellation (3)}

Das Abkommen von der Fahrbahn nach rechts ist wie auf Landstraßen auch für die Fahrunfälle in Kurven innerorts die bestimmende Unfallart (47 \% aller innerörtlichen
Fahrunfälle in Kurven). Wird die Beteiligung (Alleinunfall) und die Unfallschwere $(U(P))$ als weitere Kriterien wie im Außerortsfall hinzugezogen, ergeben sich für diese Unfallkonstellation acht Unfälle.

Die Unfallschwere ist mit einem Anteil der Unfälle mit schwerem Personenschaden von 63 \% im Vergleich zu den anderen Innerortskonstellationen mit Abstand am höchsten. Im Gegensatz zu der Situation auf LandstraBen weist keiner der Unfälle einen Aufprall auf ein Hindernis neben der Fahrbahn auf. Der Anteil der Unfälle am Wochenende ist mit einem Unfall gering. Auffällig ist, dass sich die restlichen Unfälle an einem Donnerstag oder Freitag ereigneten. Ungünstige Fahrbahnverhältnisse (nass/winterglatt/schlüpfrig) waren in keinem Fall zu verzeichnen. 38 \% der Unfälle bei Dunkelheit lassen den Einfluss schlechter Lichtverhältnisse für diese Unfallkonstellation nicht ausschließen. Die Analyse der Zielgruppenanteile der beteiligten Fahrer des PTW ergibt Auffälligkeiten bei den Frauen und Senioren. Die Unfallzahlen sind jedoch sehr gering.

Aus der Auswertung der Straßeneigenschaften resultiert ein hoher Anteil an Unfällen auf Abschnitten mit den folgenden Kriterien:

- erhebliche Mängel im Straßenzustand (25\%)

- angrenzende Bebauung lückenhaft oder keine angrenzende Bebauung (38\%)

- mindestens vier Knotenpunkte pro Kilometer (38\%).

Im Vergleich zu allen Innerortsunfällen der besichtigten Strecken sind keine Auffälligkeiten bei bestimmten StraBeneigenschaften festzustellen.

\begin{tabular}{|l|l|}
\hline Alleinunfall in Innerortskurve mit Abkommen von der Fahrbahn nach rechts (i. d. R. Linkskurve) \\
\hline Unfallkollektiv & 8 \\
\hline Unfallschwere: Anteil U (SP) & $63 \%$ \\
\hline Anteil Hauptverursacher PTW & $100 \%$ \\
\hline Anteil Unfälle am Wochenende & $13 \%$ \\
\hline Auffälligkeiten Unfallauswertung & $\begin{array}{l}\text { - kein Aufprall auf Hindernisse neben der Fahrbahn } \\
\text { - hoher Anteil der Unfälle bei Dunkelheit }\end{array}$ \\
\hline
\end{tabular}

Tabelle 7-3: Übersicht zur Unfallkonstellation (3) 


\subsection{Unfallkonstellation(4)}

\begin{tabular}{|c|c|}
\hline \multicolumn{2}{|c|}{ Einbiegen/Kreuzen-Unfall innerorts an Kreuzungen oder Einmündungen } \\
\hline Unfallkollektiv & 61 (TU Dresden / 135 (TU Berlin) \\
\hline Unfallschwere Anteil U (SP) & 25 \% (TU Dresden) / 76 \% (TU Berlin) \\
\hline Anteil Hauptverursacher PTW & $25 \%$ (TU Dresden) / $20 \%$ (TU Berlin) \\
\hline Anteil Unfälle am Wochenende & $11 \%$ (TU Dresden) / 50 \% (TU Berlin) \\
\hline auffällige Zielgruppen & Fahranfänger, Jugendliche \\
\hline Auffälligkeiten Unfallauswertung & $\begin{array}{l}\text { - typischerUnfallablauf: vorfahrtberechtigtes PTW von links } \\
\text { kollidiert mit linkseinbiegendem Pkw } \\
\text { - spezielles Problem an Einmündungen mit PTW, die auf der Vor- } \\
\text { fahrtstraße bei Stau in der 2. Reihe vorbei fahren } \\
\text { - über 1/4 der Unfälle bei Dämmerung/Dunkelheit } \\
\text { - 1/4 der Unfälle an LSA (Rotüberfahrten) }\end{array}$ \\
\hline $\begin{array}{l}\text { Auffälligkeiten Straßenraum: } \\
\text { überdurchschnittlicher Anteil } \\
\text { von Unfällen auf Abschnitten }\end{array}$ & $\begin{array}{l}\text { - mit Parkbuchten am Fahrbahnrand ( } 23 \% \text { vs. } 14 \% \text { ) } \\
\text { - mit Straßenbahn auf der Fahrbahn (44\% vs. } 38 \% \text { ) } \\
\text { - mit kreuzenden Gleisanlagen (36 \% vs. } 27 \% \text { ) }\end{array}$ \\
\hline Auffälligkeiten Fahrzeugtechnik & $\begin{array}{l}\text { frontaler Hauptanstoss in die Fahrerseite des Unfallgegners } \\
\text { mit hoher, mittlerer Verletzungsschwere von MAIS 2,44 }\end{array}$ \\
\hline
\end{tabular}

Tabelle 7-4: Übersicht zur Unfallkonstellation (4)

Die Unfallkonstellation beinhaltet alle EK-Unfälle an Kreuzungen oder Einmündungen innerorts, die mit der Unfallart 5 einhergehen.

\section{Unfallgeschehen und Straßeneigenschaften}

Etwa 1/4 der Unfälle haben einen schweren Personenschaden zur Folge, rund 3/4 einen Personenschaden. Verglichen mit der analogen Konstellation außerorts ist zu erkennen, dass die Unfallschwere deutlich abnimmt. Als Grund ist hier vor allem die im Mittel höhere Annäherungsgeschwindigkeit der Fahrzeuge auf der übergeordneten Fahrbahn an den Knotenpunkt anzunehmen. Der Anteil der Unfälle, die sich an einer LSA in Betrieb ereignen, beträgt $26 \%$. In 25 \% der Fälle ist der PTW Hauptverursacher nach VUA. Nur $11 \%$ der Unfälle fallen auf das Wochenende. Somit ist diese Konstellation schwerpunktmäßig durch einen vorfahrtgeregelten Knotenpunkt gekennzeichnet, an welcher dem werktäglich verkehrenden Fahrer des PTW auf der übergeordneten Straße die Vorfahrt genommen wird.
$28 \%$ der Unfälle ereignete sich bei ungünstigen Lichtverhältnissen. Der Wert befindet sich über dem Durchschnitt der Unfälle auf allen in Sachsen festgelegten Innerortsabschnitten (19\%). Ein nasser oder winterglatter Fahrbahnzustand aufgrund der Witterungsverhältnisse ist für das Unfallgeschehen von geringer Bedeutung (13\% der Unfälle).

Die Detailauswertung der Unfalltexte wurde für die EKUnfälle an Einmündungen und Kreuzungen getrennt durchgeführt, da sich bei separater Betrachtung ein deutlicheres Bild im Fall der Einmündungen ergibt:

- für die sechs Unfälle an Einmündungen mit LSA in Betrieb:

- eine Rotüberfahrt eines Pkws

- eine Rotüberfahrt eines Mopeds

- bei den restlichen zwei Unfällen war das Vorhandensein der LSA für den eigentlichen Unfallhergang nicht relevant - zwei Unfalltexte nicht verfügbar 
- für die 28 Unfälle an vorfahrtgeregelten Einmündungen bzw. mit abgeschalteter LSA:

- Bei 15 Unfällen lässt sich anhand der Unfalltexte ein typischer Unfallhergang ableiten: Pkw oder Lkw (als Hauptverursacher nach VUA) kommen aus der untergeordneten Zufahrt und wollen nach links auf die Vorfahrtstraße einbiegen. Dabei wird das von links kommende PTW nicht oder zu spät wahrgenommen und es kommt zum Zusammenstoß oder Sturz des PTW. Sechs dieser Unfälle ereigneten sich bei schlechten Lichtverhältnissen (Dämmerung/ Dunkelheit). Hier ist ein Einfluss auf die - im Vergleich zum Pkw/Lkw ohnehin schon schlechtere - Erkennbarkeit des PTW anzunehmen. Innerhalb dieser 15 Unfälle ist eine spezielle Unfallkonstellation enthalten, die keinen Einzelfall darstellt: Bei fünf Unfällen trat folgende Situation auf: Die übergeordnete Straße wies in mindestens einer Richtung Stau oder stockenden Verkehr auf. Dem linkseinbiegenden Pkw oder Lkw der untergeordneten Straße wurde durch ein in der Kolonne stehendem Fahrzeug eine Einbiegemöglichkeit signalisiert. Bei der Realisierung dieses Manövers näherte sich ein in zweiter Reihe an der Kolonne vorbeifahrendes PTW, wodurch es zum Unfall kam.

- Weitere sechs Unfälle splitten sich hinsichtlich des Unfallherganges derartig auf:

+ bei einem Unfall eine ähnliche Konstellation wie bei den gerade beschriebenen 15 Unfällen mit dem Unterschied, dass für den Pkw auf der untergeordneten Straße nicht hervorgeht, ob er nach links oder rechts einbiegen wollte

+ bei drei Unfällen eine ähnliche Konstellation wie bei den gerade beschriebenen 15 Unfällen mit dem Unterschied, dass der Fahrer des PTW auf der übergeordneten Straße von rechts kommt + bei zwei Unfällen war das PTW Hauptverursacher und kam aus der untergeordneten Zufahrt

- Für sechs Unfälle lagen keine Unfalltexte vor bzw. wurden in diesen keine näheren Angaben zum Unfallhergang gemacht (i.d.R. Unfälle mit sonstigem Sachschaden).

- bei einem Unfall falsche Zuweisung der Örtlichkeit (Unfall nicht an Einmündung).
Für Einmündungen lässt sich eindeutig eine typische Unfallsituation ableiten:

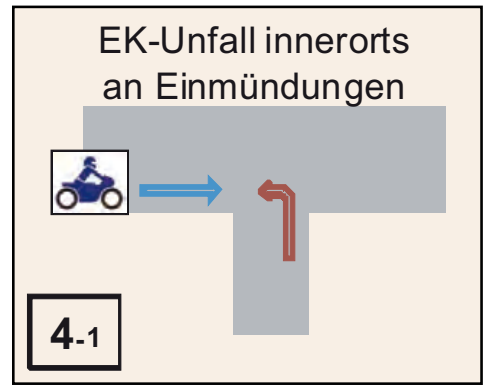

Abbildung 7-3: Typische Unfallsituation zur Unfallkonstellation (4-1) - Einmündungen

Die Auswertung der EK-Unfälle an Kreuzungen ergab hinsichtlich des Unfallherganges folgende Ergebnisse:

- für die zehn Unfälle an Kreuzungen mit LSA in Betrieb:

- fünf Rotüberfahrten des Pkw-/Lkw-Fahrzeugführers

- drei Rotüberfahrten eines PTW-Fahrers

- bei einem Unfall war das Vorhandensein der LSA für den eigentlichen Unfallhergang nicht relevant - ein AB-Unfall (falsche Zuweisung des Unfalltyps)

- für die 17 Unfälle an vorfahrtgeregelten Kreuzungen bzw. mit abgeschalteter LSA:

(1) In vier Fällen war der Pkw/Lkw Linkseinbieger auf die übergeordnete Straße, das PTW kommt von links, es kommt zum Zusammenstoß.

(2) In einem Fall wie unter (1) beschrieben, mit dem Unterschied, dass das PTW sich von rechts an den Knoten annähert.

(3) In zwei Fällen kreuzt der Pkw die übergeordnete Straße, das PTW kommt von links.

(4) In drei Fällen wie unter (3) beschrieben, mit dem Unterschied, dass das PTW von rechts ankommt.

(5) In drei Fällen war der PTW Hauptverursacher nach VUA und kam aus einer untergeordneten Zufahrt.

(6) Für vier Unfälle lagen keine Unfalltexte vor bzw. wurden in diesen keine näheren Angaben zum Unfallhergang gemacht (i. d. R. Unfälle mit sonstigem Sachschaden). 
Es kann beobachtet werden, dass die Bandbreite anzutreffender Bewegungsvorgänge bei den EK-Unfällen an vorfahrtgeregelten Kreuzungen zahlreicher und auch ausgeglichen in ihrer Auftretenshäufigkeit als im Fall der Einmündungen ist. Insgesamt bleibt jedoch auffällig, dass der Fahrer des PTW selten als Hauptverursacher auftritt und es keine Unfälle mit rechts auf die übergeordnete Straße einbiegenden Pkw/Lkw gibt. Die Unfallsituation, dass das PTW in zweiter Reihe auf der übergeordneten Straße verkehrte und von dem einbiegenden oder kreuzenden Fahrzeug übersehen wurde, ließ sich anhand der Unfalltexte ausschließlich zweimal feststellen. Schlechte Lichtverhältnisse als Einflussfaktor treten hauptsächlich bei den Unfällen an Vorfahrtkreuzungen auf. An derartigen Knoten ereigneten sich acht der insgesamt neun Unfälle bei Dämmerung/Dunkelheit. Dabei fanden die drei Unfälle, bei denen der Fahrer des PTW in der VUA als Hauptverursacher eingestuft wurde, ausnahmslos bei Dunkelheit bzw. Dämmerung statt. Möglicherweise besteht bei diesen Lichtverhältnissen ein Sichtproblem aufgrund des Tragens von (bestimmten) Helmen.

Die Untersuchung der Zielgruppen zeigt, dass insbesondere die jugendlichen Fahrer des PTW und Fahranfänger mit $22 \%$ bzw. $20 \%$ eine hohe Beteiligung aufweisen. Werden nur die Fahrer des PTW betrachtet, die als Hauptverursacher in der VUA geführt sind, erhöhen sich die Anteile auf $47 \%$ bzw. $27 \%$. Acht dieser 17 Fahrer des PTW stammen aus der Zielgruppe der Jugendlichen sowie zwei weitere Fahrer aus der Gruppe der unter 20jährigen. Damit liegt der Anteil der Jugendlichen deutlich höher als für alle Unfälle dieser Konstellation. Fünf dieser unter 20jährigen Fahrer haben ihren Führerschein noch nicht länger als zwei Jahre und gelten somit als Fahranfänger. Bei den restlichen Jugendlichen ist eine „0“ im Euska-Datensatz zu finden und damit keine Rückschlüsse auf das Alter der Fahrerlaubnis möglich. Es kann jedoch von einem beträchtlichen Überschneidungsanteil der beiden Zielgruppen ausgegangen werden, da nur ein „0“-Eintrag bei einem Fahrer des PTW älter als 20 Jahre auftrat.

Für den restlichen Teil der Unfälle mit Fahrer des PTW als Hauptverursacher ist festzustellen, dass sich die Fahrer des PTW zwischen einem Alter von 33 und 51 Jahren so- wie einem Alter der Fahrerlaubnis von 12-22 Jahren bewegen. Hier kann möglicherweise von Wiedereinsteigern ausgegangen werden. Auffällig ist, dass sich zwischen dieser Gruppe und der Gruppe sehr junger Fahrer kein anderer Fahrer des PTW als Hauptverursacher befindet.

Bei den zwei der insgesamt 13 Schlechtwetterunfälle mit einem Fahrer des PTW als Hauptverursacher entstammen beide der Zielgruppe der Jugendlichen. Bei den restlichen 11 Schlechtwetterunfällen liegt der Anteil dieser Zielgruppe bei $36 \%$ (4 Unfälle) und somit über dem Wert für alle Unfälle der untersuchten Konstellation (27\%).

Die Verknüpfung des Unfallgeschehens mit den Straßeneigenschaften zeigt die in Tabelle 8-4 beschriebenen Auffälligkeiten. Werden die EK-Unfälle an Einmündungen und Kreuzungen getrennt betrachtet, ergeben sich für die auffälligen Merkmale noch deutlichere Unterschiede im Vergleich zu den mittleren Werten aus allen Unfällen der besichtigten Streckenabschnitte. Im Fall der Einmündungen ist dann vor allem der Unfallanteil auf Abschnitten mit Parkflächen am Fahrbahnrand auffällig (29 \% vs. 14 $\%$ im Mittel). Der Umstand begünstigt ein spätes Wahrnehmen des nahenden Fahrers eines PTW auf der übergeordneten Fahrbahn. Im Fall der Kreuzungen sind es vor allem folgende Auffälligkeiten:

- 44 \% der Unfälle auf Abschnitten mit kreuzenden Gleisanlagen

- 48 \% der Unfälle auf Abschnitten mit Straßenbahn auf der Fahrbahn

- 78 \% der Unfälle auf Abschnitten mit lückenhafter oder keiner angrenzenden Bebauung

\section{Unfallgeschehen und Fahrzeugtechnik}

Bei Betrachtung der GDV-Daten zeigt sich insbesondere die im Mittel geringere Unfallschwere als bei den EK-Unfällen an Einmündungen außerorts. Rollerfahrer sind mit einem etwa doppelt so hohen Anteil gegenüber ihrem Auftreten in den anderen Unfallkonstellationen überrepräsentiert. Bei Unfällen unter Einfluss einer überhöhten Geschwindigkeit führt dies wie bei der Außerortskonstellation zu einer höheren Verletzungsschwere, allerdings weniger deutlich. 


\subsection{Unfallkonstellation (5)}

Abbiegeunfälle an Einmündungen mit der Unfallart 3 Zusammenstoß mit einem Fahrzeug, das seitlich in gleicher Richtung verkehrt - bilden die Unfallkonstellation (5).

\section{Unfallgeschehen und Straßeneigenschaften}

Die Unfallschwere nimmt im Vergleich zu den EK-Unfällen an Einmündungen etwas ab. Bei nur einem Unfall ist der Fahrer des PTW Hauptverursacher nach VUA und ausschließlich ein Unfall ereignet sich an einer LSAgeregelten Einmündung. Die Unfallsituation tritt i. d. R. werktags auf, nur zwei der neun Unfälle passierten am Wochenende. Die Auswertung der Unfalltexte ergab folgenden typischen Unfallhergang (bei 6 von 8 Unfällen an Vorfahrtknoten):

- Unfallgegner (5xPkw, 1xRad) biegen von der übergeordneten Straße nach links ab und kollidieren mit einem seitlich linkerhand in die gleiche Richtung fahrenden PTW. Dieses befindet sich entweder gerade in einem Überholvorgang oder verkehrt auf einem weiteren Fahrstreifen für diese Richtung (ein Fall). Das linksabbiegende Fahrzeug zeigt dabei in mehreren Fällen zu spät seinen Abbiegewunsch an oder biegt verbotswidrig links ab. In einem Fall überholt das Krad verbotswidrig.

Der typische Unfallhergang ist in Situation 4 der in [Kramlich 2002] definierten fünf gefährlichen Begegnungen von Pkw und PTW enthalten: Der Fahrer des PTW über-

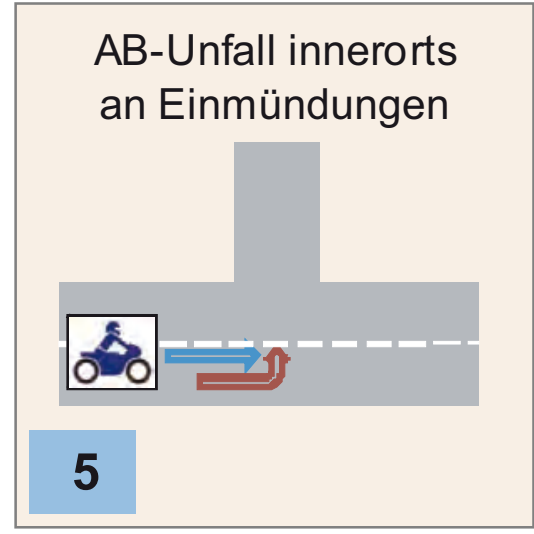

Abbildung 7-4: Typische Unfallsituation zur Unfallkonstellation (5) holt, während der Pkw abbiegt oder den Fahrstreifen wechselt. Die verbleibenden drei Unfälle sind:

- 2 Kollisionen, die ebenfalls von linksabbiegenden Pkw der übergeordneten Straße verursacht wurden (ein Fall: überholender Pkw 02 weicht seitlich aus, so dass PTW 03 in den Unfall verwickelt wird, ein Fall: Pkw 01 fährt überraschend wieder nach rechts und kollidiert mit dem seitlich rechterhand in die gleiche Richtung fahrenden PTW)

- Unfall bei einer Einmündung mit LSA in Betrieb, Kollision zweier PTW auf einem Rechtsabbiegestreifen (01 überholt den rechtsabbiegenden 02).

Der Blick auf die Zielgruppen zeigt mit Ausnahme für die weiblichen Fahrer keine Auffälligkeiten, aufgrund der kleinen Fallzahl lässt sich der Zufall als Grund dafür nicht ausschließen. Aus der Analyse bestimmter Straßeneigenschaften resultieren vier Einflussfaktoren, die in Tabelle 85 aufgeführt sind. Für Straßenabschnitte mit einem straßenbündigen Bahnkörper in Mittellage ist ein höherer Anteil an Knoten mit Linksabbiegeverboten als im Mittel zu erwarten sowie die Möglichkeit gegeben auf der Schiene zu überholen. Dies begünstigt Konfliktsituationen, aus denen Unfälle des oben beschriebenen typischen Unfallherganges entstehen können. Werden Straßeneigenschaften erhebliche Mängel im Straßenzustand und straßenbündiger Bahnkörper in Mittellage auf ein kombiniertes Auftreten überprüft, ergibt sich ein Anteil von 56 \% der Unfälle, die sich auf derartigen Abschnitten ereigneten. Für diese ist zudem festzustellen, dass es sich in allen Fällen um eine lückenhafte Bebauung handelt.

\section{Unfallgeschehen und Fahrzeugtechnik}

Auf Seite der Fahrzeugtechnik wurde eine hohe Unfallschwere für die Unfälle festgestellt, bei denen der PTWFahrer frontal in die Seite des linksabbiegenden Pkw fährt. Diese fällt bei entsprechenden Streifkollisionen deutlich geringer aus. Sportmaschinen-, Chopper- und Endurofahrer weisen mit mittleren MAIS-Werten von 2,41-2,77 höhere Verletzungsschweren in dieser Unfallkonstellation auf als Tourer-, Naked Bike- und Rollerfah$\operatorname{rer}(1,71-2,00)$. 


\begin{tabular}{|l|l|}
\hline \multicolumn{2}{|l|}{ Abbiegeunfall innerorts an Einmündungen mit seitlich in gleicher Richtung verkehrenden Kfz } \\
\hline Unfallkollektiv & 9 (TU Dresden) / 57 (TU Berlin) \\
\hline Unfallschwere Anteil U (SP) & $11 \%$ (TU Dresden) / 75\% (TU Berlin) \\
\hline Anteil Hauptverursacher PTW & $11 \%$ (TU Dresden /21 \% (TU Berlin) \\
\hline Anteil Unfälle am Wochendende & $22 \%$ (TU Dresden) / $68 \%$ (TU Berlin) \\
\hline Auffälligkeiten Unfallauswertung & $\begin{array}{l}\text { typischer Unfallablauf: PkW zeigt spät Abbiegewunsch an oder } \\
\text { biegt trotz Verbot ab.; PTW überholt im Knotenpunktbereich }\end{array}$ \\
\hline $\begin{array}{l}\text { Auffälligkeiten Straßenraum: } \\
\text { überdurchschnittlicher Anteil } \\
\text { von Unfällen auf Abschnitten }\end{array}$ & $\begin{array}{l}\text { - mit Straßenbahn auf der Fahrbahn (67\% vs. 38\%) } \\
\text { - mit erheblichen Mängeln am Straßenzustand (56 \% vs. 38 \%) } \\
\text { - mit keiner oder lückenhafter angrenzender Bebauung (78\% vs. } 70 \%) \\
\text { - mit einer Knotendichte von e“4 Knoten pro km (78\% vs. 62\%) }\end{array}$ \\
\hline Auffälligkeiten Fahrzeugtechnik & $\begin{array}{l}\text { - frontaler Hauptanstoss in die Fahrerseite des Unfallgegners mit hoher, } \\
\text { mittlerer Verletzungsschwere von MAIS 2,47 } \\
\text { - erhöhtes Unfallaufkommen am Wochenende }\end{array}$ \\
\hline
\end{tabular}

Tabelle 7-5: Übersicht zur Unfallkonstellation (5)

\subsection{Unfallkonstellation (6):}

Bei den Abbiegeunfällen an Kreuzungen traten die Unfallart 5 - Zusammenstoß mit einem Fahrzeug, das einbiegt oder kreuzt - und die Unfallart 4 - Zusammenstoß mit einem entgegenkommenden Fahrzeug - besonders hervor. Hierfür wurde zuerst jeweils eine Unfallkonstellation gebildet, die nach Auswertung der Unfalltexte zu einer zusammengefasst werden konnte. Beim Lesen der einzelnen Unfallhergänge stellte sich heraus, dass die Unfallart 5 in nahezu allen Fällen durch die Polizeibeamten falsch zugewiesen wurde und durch die Unfallart 4 ersetzt werden muss.

\section{Unfallgeschehen und Straßeneigenschaften}

Damit ergeben sich für die vorliegende Unfallkonstellation 21 Unfälle auf Seiten der TU Dresden. Die Schwere der Unfälle nimmt im Vergleich zur vorangegangenen Konstellation etwas zu und liegt in der Größenordnung von Konstellation (4). Der Anteil Fahrer von PTW als Hauptverursacher bleibt auf einem geringen Niveau (14 \%) und ein Werktag der typische Unfalltag. Auffällig ist bei dieser Konstellation der hohe Anteil von Unfällen an in Betrieb befindlichen LSA-Kreuzungen. Bei Betrachtung der Verteilung der Unfälle über die Monate ist eine leichte Häufung im Ferienmonat August zu beobachten.
Aus den Auswertungen der Unfalltexte wurden weitere Informationen zum Unfallhergang gewonnen und es konnte eine eindeutige typische Unfallsituation herausgearbeitet werden:

- Bei 17 der 21 Unfälle übersah der linksabbiegende Pkw als Hauptverursacher (in einem Fall möglicherweise ein Lkw) den aus der Gegenrichtung kommenden Fahrer des PTW. In 10 Fällen handelte es sich dabei um Kreuzungen mit LSA-Regelung in Betrieb, aber nach Interpretation der Unfalltexte ohne Sicherung der Linksabbieger durch eine eigene Phase. Jeweils fünf der 17 Unfälle geschahen bei Nässe bzw. dunklen Lichtverhältnissen. In einem Fall trat eine Überlagerung beider Unfallumstände auf.

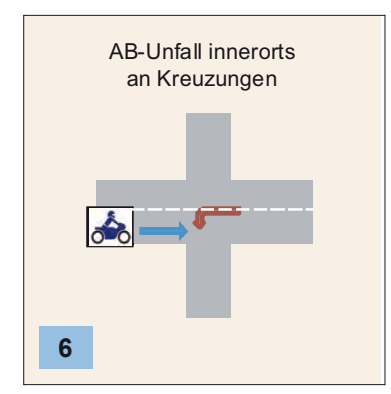

Abbildung 7-5: Typische Unfallsituation zur Unfallkonstellation (6) 


\begin{tabular}{|l|l|}
\hline \multicolumn{2}{|l|}{ Abbiegeunfall innerorts an Kreuzungen mit entgegenkommenden Kfz } \\
\hline Unfallkollektiv & 21 (TU Dresden) / 39 (TU Berlin) \\
\hline Unfallschwere Anteil U (SP) & $24 \%$ (TU Dresden) / 88 \% (TU Berlin) \\
\hline Anteil Hauptverursacher PTW & $14 \%$ (TU Dresden) / $33 \%$ (TU Berlin) \\
\hline Anteil Unfälle am Wochenende & $15 \%$ (TU Dresden) / $20 \%$ (TU Berlin) \\
\hline auffällige Zielgruppen & Jugendliche, Schlechtwetterunfälle \\
\hline Auffälligkeiten Unfallauswertung & $\begin{array}{l}\text { - hoher Anteil der Unfälle an LSA in Betrieb (62 \%) } \\
\text { - typischer Unfallablauf: Pkw ist Linksabbieger, PTW vorfahrtberechtigter } \\
\text { Geradeausfahrer }\end{array}$ \\
\hline $\begin{array}{l}\text { Auffälligkeiten Straßenraum: } \\
\text { überdurchschnittlicher Anteil } \\
\text { von Unfällen auf Abschnitten }\end{array}$ & $\begin{array}{l}\text { - mit Straßenbahn auf der Fahrbahn (52 \% vs. 38 \%) } \\
\text { - mit einer Knotenpunktdichte e“4 Knoten pro km (71 \% vs. 62 \%) } \\
\text { - mit einer zulässigen Höchstgeschwindigkeit > 50 km/h (24\% vs. 11\%) }\end{array}$ \\
\hline Auffälligkeiten Fahrzeugtechnik & $\begin{array}{l}\text { frontaler Hauptanstoss in die Fahrerseite des Unfallgegners } \\
\text { mit hoher, mittlerer Verletzungsschwere von MAIS 2,5 }\end{array}$ \\
\hline
\end{tabular}

Tabelle 7-6: Übersicht zur Unfallkonstellation (6)

- Die restlichen vier Fälle teilen sich dergestalt auf: - zwei Unfälle mit der Konstellation wie bei den 17 obigen Unfällen, mit dem Unterschied, dass der Fahrer des PTW der Linksabbieger und damit auch Hauptverursacher nach VUA ist (jeweils bei LSA in Betrieb)

- eine Rotüberfahrt des PTW-Fahrers, der dabei mit einem verbotswidrig abbiegenden Fahrzeug kollidiert - in einem Fall falsche Zuordnung der Unfallart (müsste nach Unfalltext Unfallart 3 sein, Fahrer des PTW ist hierbei Hauptverursacher nach VUA).

Bei den Zielgruppen sind vor allem die Jugendlichen mit $23 \%$ und die Schlechtwetterunfälle mit $36 \%$ stark vertreten. Werden 18jährige Fahrer mit zu den Jugendlichen gerechnet, erhöht sich deren Anteil auf 36 \%. Die vier Fahrer des PTW, welche als Hauptverursacher auftreten, stammen entweder aus einer jungen oder einer älteren Altersgruppe $(2 \times 16$ Jahre und $1 \times 20$ Jahre, $1 \times 62$ Jahre). Die Schlechtwetterunfälle teilen sich in fünf Unfälle bei nassem Straßenzustand und drei Unfällen im Monat November auf. Von diesen acht Unfällen sind die Hälfte Unfälle mit Fahrern von PTW aus der Zielgruppe der Jugendlichen.
Die Untersuchung von Straßeneigenschaften in Zusammenhang mit der vorliegenden Unfallkonstellation ergibt die in Tabelle 7-6 beschriebenen Auffälligkeiten. Zusätzlich ergibt sich mit 38 \% ein hoher Anteil von Unfällen auf Abschnitten mit erheblichen Mängeln im Straßenzustand, der aber nur geringfügig über dem Durchschnittswert liegt (36\%). Das Merkmal Straßenbahn mit straßenbündiger Führung ist wie in der vorangegangenen Konstellation als wesentlicher Einflussfaktor abzuleiten. Im GDVForschungsprojekt „Sicherheitsgrad von Stadtstraßen“ [Baier et al. 2007] wurden Sicherheitsuntersuchungen zu bestimmten Querschnittstypen mit und ohne Straßenbahn durchgeführt. Beim Vergleich der UKR weisen die Querschnitte mit straßenbündigem Bahnkörper (zum Teil deutlich) höhere Werte auf als Querschnittstypen mit besonderem Bahnkörper oder Querschnitte ohne Straßenbahn. Offen bleibt, ob die ungünstige Wirkung auf die Sicherheit von straßenseitigen Merkmalen resultiert, die mit dem Auftreten von Straßenbahnverkehr verbunden sind, oder direkt durch den Straßenbahnverkehr bzw. die damit verbundene Infrastruktur entsteht. Eine hohe Knotenpunktdichte spielt für die Unfallkonstellation ebenfalls eine Rolle. Als neuer auffälliger Faktor kommt die Geschwindigkeit hinzu, immerhin ein Viertel der Unfälle be- 
findet sich auf Abschnitten mit einer zulässigen Höchstgeschwindigkeit von mehr als $50 \mathrm{~km} / \mathrm{h}$. Die Unfälle bei schlechtem Straßenzustand traten in fünf Fällen kombiniert mit Straßenbahn auf der Fahrbahn in Mittellage und in drei Fällen kombiniert mit nassem Straßenzustand, nie zusammen mit ungünstigen Lichtverhältnissen auf. Die Unfälle auf Abschnitten mit einer zulässigen Höchstgeschwindigkeit von mehr als $50 \mathrm{~km} / \mathrm{h}$ standen in zwei Fällen mit einem schlechten Straßenzustand sowie in zwei Fällen mit dunklen Lichtverhältnissen in Zusammenhang.

\section{Unfallgeschehen und Fahrzeugtechnik}

Der Anteil der getöteten und schwerverletzten PTW-Fahrer ist in dieser Konstellation gegenüber den drei anderen Innerortskonstellationen am größten. Fahrer von Naked Bikes haben eine erhöhte Verletzungsschwere mit einem Median des MAIS-Wertes von 3.

\subsection{Unfallkonstellation (7)}

Unfälle im Längsverkehr an Kreuzungen, Einmündungen oder auf Gefällestrecken mit der Unfallart 2 - Zusammenstoß mit einem vorausfahrenden oder wartenden Fahrzeug - bilden mit 36 Unfällen die vorliegende Unfallkonstellation. Im Vergleich zu den bisherigen Konstellationen ist eine geringere Unfallschwere vorzufinden. $8 \%$ der Unfälle hatten einen schweren und $33 \%$ einen leichten Personenschaden zur Folge. Der Anteil der Unfälle an LSA-Knoten in Betrieb beträgt $40 \%$. Der Anteil der Unfälle werktags ist wie bei fast allen Innerortskonstellationen mit $83 \%$ hoch. Nasser oder winterglatter Fahrbahnzustand sowie ungünstige Lichtverhältnisse können als unfallbegünstigende Umstände mit einem Anteil von 8 \% bzw. $6 \%$ der Unfälle ausgeschlossen werden. 28 der 36 Unfälle dieser Konstellation wurden laut VUA von Fahrern des PTW verursacht. Die Auswertung der Unfalltexte zeigt für die 28 Unfälle folgende Erkenntnisse:

- In 23 Fällen kollidiert der Fahrer des PTW mit dem verkehrsbedingt haltenden oder abbremsenden $\mathrm{Kfz}$, in fast allen Fällen ein Pkw. Als Ursache kann aus dem Unfalltext entweder Unachtsamkeit bzw. ein voraus- gegangener Sturz des PTW abgeleitet werden. Stürze, in deren Folge das PTW auf ein vor ihm stehendes oder fahrendes $\mathrm{Kfz}$ rutscht, resultierten im Einzelfall nach Angaben der VUA aus: Sand, Öl oder Nässe auf der Fahrbahn, Pfeilmarkierung auf dem Fahrstreifen und zu starkes Abbremsen des PTW. Der typische Unfallhergang gestaltet sich demnach wie folgt:

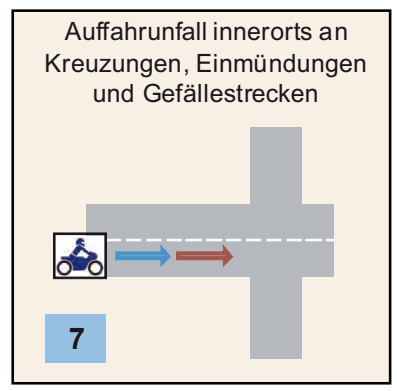

Abbildung 7-6: Typische Unfallsituation zur Unfallkonstellation (7)

- Weitere drei Unfälle stellen Besonderheiten dar:

- ein Unfall in einer Bikerkolonne

- ein Unfall bei mittigem Durchfahren zweier in derselben Richtung aufgrund einer rot zeigenden LSA wartenden Fahrzeugreihen (Unfallart nicht korrekt zugewiesen)

- ein Unfall, bei dem das PTW das vorausfahrende Fahrzeug rechts überholt (Unfallart nicht korrekt zugewiesen)

- Für zwei Fälle liegen keine vollständigen Unfalltexte vor.

Bei den acht Unfällen (davon steht bei einem der Unfalltext nicht zur Verfügung), in denen der Pkw Hauptverursacher ist, handelt es sich um Auffahrunfälle, bei denen der Pkw auf das verkehrsbedingt haltende oder abbremsende PTW auffährt. Diese Unfälle fallen schwerer aus als die Unfälle mit dem Fahrer des PTW als Hauptverursacher: $75 \%$ gegenüber $32 \%$ sind Unfälle mit Personenschaden.

Die Analyse der definierten Zielgruppen zeigt als eine wesentliche Problemgruppe die Fahranfänger mit $25 \%$ Anteil an den beteiligten Fahrern von PTW. Von den neun Fahranfängern sind mindestens drei gleichzeitig Jugendliche. Einträge in Form einer „0“ in 16 Datensätzen lassen das Führerscheinalter in 44 \% der Fälle offen. Die „0“ im EUSka-Datensatz lässt folgende Interpretationen zu: 


\begin{tabular}{|l|l|}
\hline \multicolumn{2}{|l|}{ Auffahrunfall innerorts an Kreuzungen, Einmündungen und Gefällestrecken } \\
\hline Unfallkollektiv & 36 \\
\hline Unfallschwere Anteil U (SP) & $8 \%$ \\
\hline Anteil Hauptverursacher PTW & $78 \%$ \\
\hline Anteil Unfälle am Wochenende & $17 \%$ \\
\hline auffällige Zielgruppen & Fahranfänger, Jugendliche, Kraftrollerfahrer \\
\hline $\begin{array}{l}\text { Auffälligkeiten } \\
\text { Unfallauswertung }\end{array}$ & $-40 \%$ der Unfälle an LSA in Betrieb \\
& - Nässe auf Fahrbahn ist sehr selten mitwirkend \\
\hline $\begin{array}{l}\text { Auffälligkeiten Straßenraum: } \\
\text { überdurchschnittlicher Anteil } \\
\text { von Unfällen auf Abschnitten }\end{array}$ & \begin{tabular}{l} 
- höhere Unfallschwere, wenn Pkw auf PTW auffährt \\
\hline
\end{tabular} \\
\hline
\end{tabular}

Tabelle 7-7: Übersicht zur Unfallkonstellation (7)

- der Jugendliche besitzt keine gültige Fahrerlaubnis

- die Fahrerlaubnis ist noch nicht ein Jahr alt

- das Merkmal wurde bei der Unfallaufnahme nicht erfasst.

Die Zielgruppe der Jugendlichen besitzt einen Anteil von $22 \%$. Werden die 18jährigen Fahrer darin eingeschlossen, ergibt sich ein Anteil von $36 \%$. Bei alleiniger Betrachtung der Unfälle mit einem PTW als Hauptverursacher erhöht sich der Anteil der Jugendlichen (einschließlich der 18jährigen Fahrer) auf $46 \%$ und der Fahranfänger noch einmal auf $29 \%$.

Mit $22 \%$ sind als weitere Zielgruppe die Schlechtwetterunfälle vertreten, davon ereigneten sich fünf der acht Unfälle in den Wintermonaten November bis März. Die Zielgruppe der Kraftrollerfahrer tritt bei dieser Konstellation das erste Mal mit mehr als einem Beteiligten auf, ist mit drei Beteiligten aber weiterhin von geringer Bedeutung. Frauen weisen mit $14 \%$ Beteiligung ebenfalls einen überdurchschnittlichen Wert auf. Der Anteil bei Kraftrollerfahrern und Frauen geht bei alleiniger Betrachtung der Unfälle mit einem PTW als Hauptverursacher im Gegensatz zu den Schlechtwetterunfällen zurück.

Bei Untersuchung der Straßeneigenschaften kann zusätzlich zu den in Tabelle 8-7 festgehaltenen Eigenschaften ein hoher Anteil von Unfällen auf Abschnitten mit Straßenbahn auf der Fahrbahn (40\%) sowie mit kreuzenden Gleisanlagen (25\%) festgestellt werden.

\subsection{Unfallkonstellation (8)}

Die 35 Unfälle dieser Unfallkonstellation weisen die geringste Unfallschwere aller untersuchten Konstellationen auf. Rund $1 / 4$ der Unfälle hat einen Personenschaden zur Folge, davon wurde in zwei Fällen ein Verunglückter schwer verletzt. $77 \%$ der Unfälle passieren werktags. Hauptverursacher ist in $60 \%$ der Fälle der Fahrer des PTW. Der Anteil der Unfälle an LSA-Knotenpunkten in Betrieb beträgt $37 \%$. Ungünstige Witterungsbedingungen und Lichtverhältnisse besitzen mit je 14 \% der Unfälle einen eher geringen Einfluss auf das Unfallgeschehen. Aus der Auswertung der Unfalltexte geht keine eindeutige typische Unfallsituation hervor:

- 11 Unfälle ereignen sich bei einem Fahrstreifenwechselvorgang, in fünf Fällen ist der Auslöser ein beabsichtigter Fahrstreifenwechsel durch das PTW als 01.

- Bei sieben Unfällen ist der Fahrer des PTW Hauptverursacher und fährt an verkehrsbedingt stehenden Fahrzeugen entweder links oder zwischen zwei Fahrzeugkolonnen mittig hindurch und beschädigt dabei ein anderes $\mathrm{Kfz}$. 
- Bei zwei Unfällen streift das überholende PTW 01 einen Pkw.

- Bei weiteren drei Unfällen ist nicht ersichtlich, ob es sich um angesetzte Überholvorgänge oder geplante Fahrstreifenwechsel handelt.

- Bei zwei Unfällen (in einem Fall an einem Knoten mit LSA in Betrieb) biegt der Pkw als Hauptverursacher rechts bzw. links ab und übersieht dabei den rechts bzw. links von hinten kommenden Fahrer des PTW.

- Bei zwei Unfällen fahren im ersten Fall zwei PTW nebeneinander und es kommt aufgrund eines Ausweichmanövers zur Kollision sowie im zweiten Fall ein Pkw (Hauptverursacher) und ein PTW nebeneinander und es kommt zur Berührung.

- Bei einem Unfall wird das PTW 02 absichtlich ausgebremst und abgedrängt.

- In einem Fall übersieht das linksabbiegende PTW 01 den gerade überholenden Pkw. Der Unfalltyp wurde hier demnach falsch zugewiesen.

- Bei einem Unfall wurde eine falsche Unfallart zugewiesen, es handelt sich eigentlich um einen Auffahrunfall (Unfallart 2).

- Für fünf Unfälle sind keine ausreichenden Angaben in der VUA zu finden.

Es zeigt sich, dass am häufigsten ein Unfall, welcher aus einem Konflikt bei Fahrstreifen-Wechselvorgängen resul- tiert, auftritt. Der Anteil beträgt insgesamt jedoch weniger als $1 / 3$ der Unfälle dieser Konstellation. Die restlichen Unfälle sind in verschiedensten Konfliktsituationen begründet.

Die Betrachtung der Zielgruppen ergibt eine Auffälligkeit bei den Kraftrollerfahrern. Deren Anteil liegt bei $8 \%$, trägt aber mit drei Unfällen keinen Hauptanteil am Unfallgeschehen. Mit $25 \%$ sind die Schlechtwetterunfälle vertreten. Bei den Beteiligten dieser neun Unfälle befand sich kein Jugendlicher, dafür zwei Kraftrollerfahrer. Werden nur die Unfälle untersucht, bei denen der PTW Hauptverursacher ist, steigt der Anteil für die Kraftrollerfahrer. Der Abfall der Unfallzahlen in den Ferienmonaten im Sommer deutet darauf hin, dass in der Konstellation eher Fahrer betroffen sind, die aus beruflichen oder Ausbildungsgründen ihr motorisiertes Zweirad nutzen.

Aus der Untersuchung der Straßeneigenschaften resultieren die in Tabelle 8-8 beschriebenen Auffälligkeiten. Sobald eine Fahrtrichtung mehr als ein Fahrstreifen aufweist, ist von dem PTW-Fahrer eine höhere Aufmerksamkeit gefordert. Werden nur die Unfälle ohne Charakteristik betrachtet (d. h. es erfolgte entweder kein Eintrag in der VUA oder die Unfälle ereigneten sich auf der freien Strecke) zeigt sich außerdem, dass $38 \%$ dieser Unfälle auf Abschnitten mit erheblichen Defiziten im Straßenzustand passiert sind. Wird allein vom Vorhandensein

\begin{tabular}{|c|c|}
\hline \multicolumn{2}{|c|}{$\begin{array}{l}\text { Unfälle mit seitlich in gleicher Richtung verkehrenden Kfz an Kreu- } \\
\text { zungen oder Einmündungensowie ohne Charakteristik innerorts }\end{array}$} \\
\hline Unfallkollektiv & $\stackrel{\Delta}{\longrightarrow}$ \\
\hline Unfallschwere Anteil U (SP) & $6 \%$ \\
\hline Anteil Hauptverursacher PTW & $60 \%$ \\
\hline Anteil Unfälle am Wochenende & $23 \%$ \\
\hline auffällige Zielgruppen & Kraftrollerfahrer \\
\hline $\begin{array}{l}\text { Auffälligkeiten } \\
\text { Unfallauswertung }\end{array}$ & $\begin{array}{l}\text { - } 37 \% \text { der Unfälle an LSA in Betrieb } \\
\text { - häufigste Unfallsituation ergibt sich bei Fahrstreifenwechselvorgängen }\end{array}$ \\
\hline $\begin{array}{l}\text { Auffälligkeiten Straßenraum: } \\
\text { überdurchschnittlicher Anteil } \\
\text { von Unfällen auf Abschnitten }\end{array}$ & $\begin{array}{l}\text { - mit mindestens } 2 \text { Fahrstreifen pro Richtung (26 \% vs. } 17 \text { \%) } \\
\text { - mit keiner oder lückenhafter angrenzender Bebauung (89\% vs. } 70 \text { \%) } \\
\text { - mit kreuzenden Gleisanlagen (43\% vs. } 27 \% \text { ) }\end{array}$ \\
\hline
\end{tabular}

Tabelle 7-8: Übersicht zur Unfallkonstellation (8) 
von Mängeln im Zustand ausgegangen, erhöht sich der Anteil auf $50 \%$. Nahezu alle Unfälle ereigneten sich auf Abschnitten mit lückenhafter oder keiner Bebauung. Weiterhin ist der Unfallanteil auf Abschnitten mit kreuzenden Gleisanlagen auffälliger (56\%) als für die Unfälle der gesamten Konstellation.

\subsection{Unfallkonstellation (9)}

Von den 18 Fahrunfällen auf besichtigten Innerortsabschnitten ohne eine besondere Charakteristik der Unfallstelle sind 12 der Unfallart 0 - Unfall anderer Art - zugeordnet. Durch Auswertung des Unfallherganges soll festgestellt werden, ob sich trotz fehlender Unfallart typische Unfallsituationen ergeben. Die Unfallschwere fällt etwas geringer aus als bei den Fahrunfällen innerorts an Kurven, liegt aber über den anderen Innerortskonstellationen. 92 \% der Unfälle haben einen Personenschaden zur Folge. Der Anteil der Unfälle an einem Werktag befindet sich mit $84 \%$ in ähnlicher Größenordnung im Vergleich zu diesen Konstellationen. Bei allen 12 Unfällen handelt es sich um Alleinunfälle. $25 \%$ passierten bei ungünstigen Lichtverhältnissen sowie $58 \%$ bei nassem Straßenzustand. Wird das Unfallmerkmal "winterglatte Fahrbahn" oder "schlüpfriger Fahrbahnzustand" hinzugenommen, ergibt sich sogar ein Anteil von 75 \% der Unfälle. Ein schlechter Fahrbahnzustand aufgrund äußerer (Witterungs-) Bedingungen lässt sich somit als ein erster unfallbegünstigender Umstand für die vorliegende Konstellation ableiten. Die Sichtung der Unfalltexte (eine VUA mit unzureichenden Angaben) ergab als weitere unfallbegünstigende Faktoren:

- ein Sturz durch Ölspur, wahrscheinlich im Beschleunigungsvorgang

- ein Sturz durch winterglatte Fahrbahn

- ein Sturz bei nasser Fahrbahn, begünstigt durch das deutliche Unterschreiten der Mindestprofiltiefe am Hinterrad

- drei Stürze beeinflusst durch das Befahren der Straßenbahnschienen, davon zwei bei nassem Straßenzustand - 5 Stürze während des (verkehrsbedingten) Bremsvorganges, davon vier bei nasser Fahrbahn.

Daraus resultiert, dass insbesondere die Kombination eines schlechten Zustandes der Fahrbahn aufgrund äußerer Witterungsbedingungen verbunden mit dem Einleiten eines Bremsvorgangs unfallbegünstigend wirkt. Das Befahren eines straßenbündigen Bahnkörpers kann durch die auftretende Veränderung der Haftreibung zu einem zusätzlichen Verlust der Haftung führen und verstärkt so das Risiko bei schlechten Witterungsverhältnissen zu verunglücken.

Die Auswertung der Zielgruppen bringt keine neuen Erkenntnisse, in dem einzig die Zielgruppe der Schlechtwetterunfälle mit $67 \%$ auffällig ist (acht Unfälle bei nas-

\begin{tabular}{|c|c|}
\hline \multicolumn{2}{|c|}{ Fahrunfall innerorts anderer Art ohne Charakteristik } \\
\hline Unfallkollektiv & 12 \\
\hline Unfallschwere Anteil U (SP) & $42 \%$ \\
\hline Anteil Hauptverursacher PTW & $100 \%$ \\
\hline Anteil Unfälle am Wochenende & $16 \%$ \\
\hline auffällige Zielgruppen & Schlechtwetterunfälle \\
\hline Auffälligkeiten Unfallauswertung & $\begin{array}{l}\text { - alle Unfälle sind Alleinunfälle } \\
\text { - } 75 \% \text { der Unfälle bei Nässe, winterglatter oder schlüpfriger Fahrbahn }\end{array}$ \\
\hline $\begin{array}{l}\text { Auffälligkeiten Straßenraum: } \\
\text { überdurchschnittlicher Anteil } \\
\text { von Unfällen auf Abschnitten }\end{array}$ & $\begin{array}{l}\text { - mit Straßenbahn auf der Fahrbahn ( } 50 \% \text { vs. } 38 \% \text { ) } \\
\text { - mit kreuzenden Gleisanlagen (42\% vs. } 27 \% \text { ) } \\
\text { - mit einer zulässigen Höchstgeschwindigkeit von mehr als } \\
50 \text { km/h (17\% vs. } 11 \% \text { ) }\end{array}$ \\
\hline
\end{tabular}

Tabelle 7-9: Übersicht zur Unfallkonstellation (9) 
ser oder winterglatter Fahrbahn, davon ein Unfall in den Wintermonaten November bis März).

Die Betrachtung bestimmter Straßeneigenschaften im Zusammenhang mit dem Unfallgeschehen bestätigt ebenfalls die bisherigen Feststellungen (siehe Tabelle 7-9).

Herauszuheben sind vor allem die Anteile der Unfälle auf Abschnitten mit Straßenbahn auf der Fahrbahn (50\%) und mit kreuzenden Gleisanlagen (42\%). Immerhin ein Viertel der Unfälle ereignete sich auf Abschnitten mit erheblichen Mängeln im Straßenzustand. Eine solche Ausprägung des Straßenzustandes verstärkt noch einmal zusätzlich den oben beschriebenen Effekt und ist damit als unfallbegünstigend einzuschätzen. Außerorts hat sich keine analoge Unfallkonstellation herausgebildet, da dort anzunehmen ist, dass bei schlechten Witterungsbedingungen kaum Ausflugsverkehr stattfindet. Innerorts handelt es sich vermutlich zu einem großen Teil um Berufsverkehr, der auch bei schlechter Witterung auf seine Maschine angewiesen ist.

\subsection{Unfallkonstellation (10)}

Das Kollektiv dieser Unfallkonstellation setzt sich aus den Unfällen im Längsverkehr ohne Eintrag bei der Charakteristik der Unfallstelle zusammen, die der Unfallart 2 zugewiesen wurden. Es besteht aus 24 Unfällen, davon sind rund 1/3 Unfälle mit Personenschaden. Unfälle bei ungünstigen Lichtverhältnissen und bei nasser oder winterglatter Fahrbahn stellen mit 4 \% bzw. 17 \% Unfallanteilen keine Auffälligkeit dar. Der Durchschnitt liegt für alle Innerortsabschnitte bei $19 \%$ bzw. $31 \%$. Obwohl den Unfällen laut VUA keine Charakteristik zugeordnet wurde, ereigneten sich 38 $\%$ der Unfälle an LSA in Betrieb. Da nicht davon ausgegan- gen werden kann, dass alle Unfälle an einer Fußgängeroder sonstigen LSA passiert sind, ist daraus zu schließen, dass bei der Unfallaufnahme in der VUA für das Merkmal Charakteristik der Unfallstelle nicht immer ein Eintrag im Fall eines Knotenpunktes als Örtlichkeit (Kreuzung, Einmündung) erfolgt. Bei 15 Unfällen war der Fahrer des PTW der Hauptverursacher. Die Auswertung der Unfalltexte ergibt, dass alle Unfälle typische Auffahrunfälle sind, welche i. d. R. durch Unachtsamkeit und einen verkehrsbedingten Halt bzw. Bremsmanöver des vorausfahrenden Fahrzeuges verursacht wurden. In einem einzigen Fall ist das auffahrende Fahrzeug nicht der Hauptverursacher nach VUA.

Die Untersuchung der Zielgruppen zeigt mit $38 \%$ einen hohen Anteil Jugendlicher an den beteiligten Fahrer von PTW, davon ist einer eindeutig zugleich Fahranfänger. Bei den restlichen acht Jugendlichen ist in der EUSka-Datenbank eine "O " für das Alter der Fahrerlaubnis eingetragen (Interpretationsmöglichkeiten siehe vorherige Unfallkonstellationen). Insgesamt existieren für die beteiligten Fahrer des PTW 13 solcher Einträge. Der Anteil der eindeutig als Fahranfänger zuzuordnenden Fahrer beträgt $13 \%$. Ein Viertel der Unfälle sind Schlechtwetter-unfälle (vier bei nassem Straßenzustand und zwei Unfälle in den Wintermonaten November bis März). Die weiteren Zielgruppen sind für diese Unfallkonstellation ohne Bedeutung.

Bei den Straßeneigenschaften ergeben sich knapp 1/3 der Unfälle auf Abschnitten mit erheblichen Defiziten im StraBenzustand sowie ähnlich hohe Anteile von Unfällen auf Abschnitten mit Straßenbahn auf der Fahrbahn und auf Abschnitten mit kreuzenden Gleisanlagen. Im Vergleich mit den Durchschnittswerten für die Unfälle aller besichtigten Strecken innerorts zeigt sich jedoch keine Auffälligkeit.

\begin{tabular}{|l|l|}
\hline Auffahrunfall innerorts ohne Charakteristik \\
\hline Unfallkollektiv & 24 \\
\hline Unfallschwere Anteil U (SP) & $4 \%$ \\
\hline Anteil Hauptverursacher PTW & $63 \%$ \\
\hline Anteil Unfälle am Wochenende & $17 \%$ \\
\hline auffällige Zielgruppen & Jugendliche \\
\hline Auffälligkeiten Unfallauswertung & $38 \%$ der Unfälle an LSA in Betrieb \\
\hline
\end{tabular}

Tabelle 7-10: Übersicht zur Unfallkonstellation (10) 


\section{Ableitung von Maßnahmen}

Die Auswertungen bezüglich der Fahrzeugtechnik (vgl. Abschnitt 6.2.9) haben gezeigt, dass Motorradunfälle in direkten Kollisionen mit einem Unfallgegner zu höheren Verletzungen des Kopfes und Thorax führen. Auch hat ein geringes Leistungsgewicht (kg/kW) unter bestimmten Randbedingungen eine höhere Verletzungsschwere zur Folge. Gerade die gemeinsame Betrachtung der Daten der Motorradfahrerbefragung und der Unfalldaten des GDV lassen erst eine eindeutige Ursachenanalyse und damit Maßnahmenfindung zu.

Im Bereich der Straße gibt das 2007 erschienene Merkblatt zur Verbesserung der Verkehrssicherheit auf Motorradstrecken [FGSV 2007] ausführliche Hinweise zur Findung von Maßnahmen, die speziell auf die Beseitigung örtlich begrenzter motorradunfallauffälliger Bereiche ausgerichtet sind. Darin sind sowohl Maßnahmen zur Unfallvermeidung als auch Maßnahmen zur Verminderung der Unfallfolgen beschrieben. In Abhängigkeit typischer Unfallsituationen, die durch Unfalltyp und Örtlichkeit festgelegt sind, und möglicher Einflussfaktoren der Straße werden kurz- und mittelfristige Maßnahmen vorgeschlagen. Dabei wird zwischen verkehrsrechtlichen, straßenbaulichen/betrieblichen Maßnahmen sowie Verkehrsüberwachungsmaßnahmen differenziert. Als erfolgversprechendes Maßnahmenkonzept wird eine Kombination von Maßnahmen aus diesen Bereichen empfohlen [FGSV 2007].

Die vom Auftragnehmer erarbeiteten Maßnahmenempfehlungen können zum Teil auch unterstützend bei der Maßnahmenwahl im Rahmen Örtlicher Unfalluntersuchungen wirken, z. B. an Stellen, an denen eine der untersuchten Unfallkonstellationen gehäuft auftritt. Sie stellen aber vor allem generelle Empfehlungen für die Gestaltung von Straßenraum und Fahrzeug sowie für Einflussmöglichkeiten auf das Nutzerverhalten dar.

\subsection{Maßnahmen zur Unfallkonstellation}

Die detaillierte Auswertung des Unfallgeschehens der Unfallkonstellationen in Zusammenhang mit be- stimmten straßenseitigen und fahrzeugtechnischen Eigenschaften ermöglicht es, für typische Unfallsituationen Maßnahmen zu finden, die zielgerichtet auf die dort auftretenden Auffälligkeiten - z. B. in Bezug auf Zielgruppen, Verletzungsmuster, Fahrzeugeigenschaft, Straßeneigenschaft - eingehen (Tabelle 8-1).

In Bezug auf die Unfallkonstellationen (1) und (3) wird speziell auf das neue Merkblatt zur Motorradsicherheit [FGSV 2007] hingewiesen. Darin wird ein Verfahren zur Auswahl von Art und Umfang senkrechter Leiteinrichtungen auf Motorradstrecken zur Verfügung gestellt, das die aktuelle Situation in der Kurve (Vorhandensein von Schutzplanke, Richtungstafel) sowie den Kurvenverlauf berücksichtigt.

\subsection{Technische Maßnahmen}

\subsubsection{Rückhaltesysteme}

Honda hat mit Einführung des aktuellen Modells der GoldWing gezeigt, dass die Übertragung der Prinzipien der passiven Sicherheit aus dem Pkw-Bereich auf den Motorradsektor Verletzungsschweren im Fall einer Kollision senken kann. Wird der direkte Kontakt des Motorradfahrers mit dem Unfallgegner oder Hindernis vermieden, so sinken die Verletzungsschweren deutlich. Der Hintergrund ist, dass der zur Verfügung stehende Vorverlagerungsweg des Motorradfahrers ausgenutzt wird, um auf einem ertragbaren Niveau kinetische Energie abzubauen. Der gefüllte Luftsack ermöglicht es so, die Belastungen von Kopf und Thorax zu reduzieren. Ein optimaler Ride-Down-Factor ist damit aber noch nicht zu erreichen. Erst wenn der Aufsasse zum frühsten möglichen Zeitpunkt zurückgehalten wird, ist die Belastung für den menschlichen Körper am geringsten. Im Pkw-Bereich ist daher in Europa der Airbag als rein unterstützendes Rückhaltesystem zum Sicherheitsgurt installiert. Die größten Rückhalteerfolge erzielt man in der Fahrzeugsicherheit mit dem frühen Teilnehmen des Menschen an der Verzögerung des Fahrzeugs. Daher sollte ein Gurtsystem etabliert werden, welches den Motorradfahrer schon im ersten Moment der Kollision zurückhält. BMW hat diese mit dem C1 Roller bereits verwirklicht. 


\begin{tabular}{|c|l|}
\hline Unfallkonstellation & Maßnahmen \\
\hline$(1)$ & $\begin{array}{l}\text { - hindernisfreier Seitenraum in der Kurvenaußenseite } \\
\text { - Beseitigung nicht erforderlicher passiver Schutzeinrichtungen } \\
\text { - Einsatz motorradfreundlicher passiver Schutzeinrichtungen }\end{array}$ \\
\hline$(2)$ & $\begin{array}{l}\text { - Überprüfung unfallauffälliger Knotenpunkte auf Erkennbarkeit, Begreifbarkeit und Sicht } \\
\text { - Beseitigung von Sichteinschränkungen } \\
\text { - Geschwindigkeittsbeschränkung und -überwachung } \\
\text { (betrifft vor allem die PTW-Fahrer auf der übergeordneten Straße) }\end{array}$ \\
\hline$(3)$ & $\begin{array}{l}\text { - Beseitigung von Fahrbahnschäden } \\
\text { - Verdeutlichung der Linienführung (besonders bei Dunkelheit) } \\
\text { - Geschwindigkeitsbeschränkung und -überwachung (betrifft vor allem die PTW-Fahrer) }\end{array}$ \\
\hline$(4)$ & $\begin{array}{l}\text { - keine Anordnung von Parkbuchten / Parkflächen auf der übergeordneten Fahrbahn } \\
\text { im Bereich von Knotenpunkten } \\
\text { - verbesserte Ausbildung von Fahranfängern (insbesondere Fahren bei Dunkelheit) }\end{array}$ \\
\hline$(5)$ & $\begin{array}{l}\text { - Anordnung von Linksabbiegestreifen auf den übergeordneten Zufahrten } \\
\text { - Schulung der PTW-Fahrer, dass im Bereich von Knotenpunkten nicht überholt werden darf } \\
\text { - Schulung des Bewusstseins für Motorradfahrer beim Unfallgegner }\end{array}$ \\
\hline$(6)$ & $\begin{array}{l}\text { - Einrichtung einer eigenen Phase für Linksabbieger } \\
\text { - Schulung des Bewussteseins für Motorradfahrer beim Unfallgegner }\end{array}$ \\
\hline - verbesserte Ausbildung von Fahranfängern (insbesondere Bremsvorgänge) \\
- Promotion Motorräder mit ABS
\end{tabular}

Tabelle 8-1: Maßnahmenvorschläge zu den 10 Unfallkonstellationen

\subsubsection{Fahrzeugstruktur}

Die Struktur des Pkw bietet längere Verformungswege, wodurch eine geringere Belastung des Fahrzeugnutzers erzielt werden kann. Eine Unterstützung der Frontstruktur des Motorradfahrers durch die Seitenstruktur des Unfallgegners kann theoretisch die Belastungen bei allen Unfallbeteiligten senken. In Folge der schmalen Silhouette des Einspurfahrzeugs führt der kleinflächige Energieeintrag in die Seitenstruktur des Unfallgegners zu besonders tiefer Intrusion. Dadurch verlängert sich auf der einen Seite der
Verzögerungsweg für das Motorrad, andererseits verringert sich durch das Eindringen der zur Verfügung stehende Freiraum des Motorradfahrers zur harten und gefährlichen Fahrzeugseitenstrukur; zudem wird der Überlebensraum des stoßzugewandten Pkw-Insassen stark vermindert. Kann sich das Motorrad gezielt in der Struktur des Unfallgegners abstützen, so wird zum einen die Intrusion minimiert, zum anderen der Abstand zwischen Motorradfahrer und Dachkante erhöht. Die Deformationskennung des Motorrads muss also weich genug sein, um nicht wesentlich in das getroffene Fahrzeug einzudringen. Damit bleibt 
aber nicht viel Potential zur Umsetzung von kinetischer Energie in Deformationsenergie. Der Aufsasse des Motorrades muss daher durch ein energiewandelndes Element wie einen Airbag verzögert werden. Da die Gefahr besteht, dass der Motorradfahrer bei vielen Unfallkonstellationen den Airbag nicht mehr oder weniger zentral trifft, kann ein zusätzliches Gurtsystem sinnvoll sein. Es verbindet den Motorradfahrer mit seiner Maschine und kann außerdem so ausgelegt werden, dass es rascher als der Airbag wirksam wird und gezielt Bewegungsenergie abbaut (vgl. 8.2.1). Da in dieser Konstellation eine Trennung des Aufsassen vom Motorrad in Verlauf eines Unfalls nicht möglich ist, muss konstruktiv dafür Sorge getragen werden, dass er auch im Sturz geschützt wird und nicht von der Maschine erschlagen werden kann.

Ein noch weiter gehender Schritt wäre die Schaffung einer geschützten Zone für den/die Aufsassen eines Motorrades. Dieser Ansatz wurde beim BMW C1 realisiert; der Erfolg war auch auf Grund konzeptioneller Schwächen nicht überzeugend. Neue Techniken wie sie z. B. heute zum Schutz der Insassen von Cabrios verwendet werden, können zu Lösungen führen, die die „klassische Anmutung" eines Motorrad weitgehend erhalten und dennoch wesentlich besseren Schutz bieten.

\subsubsection{Begrenzung des Beschleunigungsvermögens}

Das Leistungsgewicht hat einen eindeutigen Einfluss auf das Unfallgeschehen der betroffenen Motorradfahrer. Wird ihnen, wie in dichtem Verkehr, nicht die Möglichkeit gegeben in besonders kurzer Zeit eine hohe Geschwindigkeit zu erzielen, fällt das Erleben des „Thrills“ aus. Die bewusst gefährliche Fahrt findet dann nicht statt. Eine generelle Beschränkung der Leistung in Abhängigkeit vom Fahrzeuggewicht würde jedoch zu einer breiten Ablehnung in der Gruppe der Motorradfahrer führen Eine geeignete Maßnahme stellt zunächst nur die bewusste Eigenverantwortung des Motorradfahrers dar. Sie könnte durch ein Assistenzsystem unterstützt werden, das in Abhängigkeit von der Streckenführung, der Verkehrslage und anderen Einflussgrößen Empfehlungen bezüglich des Risikos starker Beschleunigung und (bezogen auf die Strecke) hoher Geschwindigkeit macht.

\subsection{Verkehrserziehungsmaßnahmen}

Der große und zugleich hochsignifikante Einfluss des Leistungsgewichts auf die Verletzungs $\neg$ schwere des Motorradfahrers kann auf verschieden Weisen abgebaut werden. Betrachtet man den Motorradfahrer und sein Motorrad als abgeschlossene Einheit, sei in extrinsische und intrinsische Maßnahmen unterschieden.

\subsubsection{Extrinsische Maßnahmen}

Die Zahl der Verkehrstoten konnte in den letzten dreiBig Jahren durch unterschiedliche Maßnahmen deutlich von über 20.000 Verkehrstoten auf ca. 5.000 Verkehrstote im Jahr 2007 gesenkt werden. Wichtigste Maßnahme war dabei die Einführung eines Bußgeldes bei Nichtanlegen des Gurts im Pkw. Aufgrund der Datenbasis lässt sich leider keine Aussage treffen, ob die Verwendung von Integralhelmen gegenüber der Verwendung von Jethelmen einen signifikanten Einfluss auf die Verletzungsschwere hat. Auch kann nicht abschließend gezeigt werden, dass die Verwendung von Protektorenkombis einen Vorteil gegenüber anderer Bekleidung bietet, doch ist zu vermuten, dass durch die Verwendung geeigneter Schutzkleidung die Zahl vermeidbarer Verletzungen reduziert werden könnten. Die Einführung eines Bußgeldes bei Nichtverwendung von Motorradfahrerschutzkleidung könnte also die Zahl und die Schwere von Verletzungen senken. Sie würde jedoch von einer starken Gruppe unter den Motorradfahrern als Bevormundung empfunden und damit abgelehnt. Eine andere Frage ist es, ob die Folgen aus einem solchen Verhalten von der Gemeinschaft getragen werden müssen oder ob dafür eine private Deckung verbindlich vorgeschrieben wird. Es stellt sich daher die Frage, ob bei Unfällen, bei denen ein bewusst eingegangenes Risiko eine Rolle spielt, stets ein Mitverschulden des Motorradfahrers angenommen werden soll. Auf dem Weg über ein höheres wirtschaftliches Risiko - Versicherungsprämie, gegebenenfalls Schadensersatzansprüche - kann zumindest bei einem Teil der Motorradfahrer Einfluss auf das Verhalten genommen werden. 


\subsubsection{Intrinsische Maßnahmen}

Weitaus einfacher gegenüber der Realisierung der passiven Sicherheit im Motorradbau ist die Stärkung der aktiven Sicherheit in Form von Fahrerschulungen in und nach der Fahrschulausbildung. Viele Motorradfahrer sind sicherheitsbewusst und nutzen jede Möglichkeit ihr Risiko im Straßenverkehr zu minimieren. In subjektiv sicheren Situationen kommt es jedoch bei einer bestimmten Gruppe von Fahrern leistungsstarker Motorräder zu einem bewussten „In Kauf Nehmen“ vermeintlich kalkulierbaren Risikos. Der Fahrer erliegt dem Reiz des Gefährlichen, geht aber davon aus, dass „ihm“ nichts passiert. Eine gezielte Fahrerausbildung kann diese Situationen erfahrbar machen und diese so in dessen Bewusstsein einprägen. Honda hat einen „Riding Trainer" vorgestellt, der Gefahrensituationen dem Motorradfahrer veranschaulichen und so sein Gespür für gefährliche Situationen schärfen soll. Neben den eigenen Überschätzungen, wird die Interaktion zwischen den Fahrzeugen im Straßenverkehr dargestellt. Lernt man mit dem Fehler der anderen zu rechnen, können viele gefährliche Situationen in der Entstehung eliminiert werden.

Auch dieser Ansatz kann durch ein Fahrerassistenzsystem unterstützt werden. Es ist vorstellbar, das Verhalten eines Motorradfahrers im Hinblick auf fahrdynamische Daten „mitzuschreiben“. Ein Anreiz für die Installation eines solchen Systems könnte in der Gewährung einer günstigeren, vielleicht individualisierten Versicherungseinstufung bestehen.

\subsection{Maßnahmen beim Unfallgegner}

Die Datenanalyse hat klar gezeigt, dass nicht nur der Motorradfahrer, sondern häufig auch der Unfallgegner die Schuld an einer Kollision trägt. Pkw-Fahrern fehlt im Allgemeinen das Bewusstsein für Motorradfahrer. Durch die schmale Silhouette werden Motorradfahrer in ihrer Geschwindigkeit und ihrer Entfernung falsch eingeschätzt (Unger, 2004) und es kommt zu den beschriebenen Kreuzungsunfällen mit schweren Folgen.
In der Führerscheinausbildung von Pkw-Fahrern sollte auf die speziellen Eigenschaften des Motorrades im Straßenverkehr eingegangen werden. Ein Sicherheitsgewinn entsteht, wenn der kreuzende, potentielle Unfallgegner weiß, dass das herannahende Motorrad sich näher und schneller an inm befindet als er schätzt und lieber auf ein Lücke hinter dem Zweirad wartet. Auch ist eine bewusste Verfolgung der Position des Motorradfahrers, besonders, wenn er oft die Spur wechselt, im gleichgerichteten Verkehr risikomindernd. 


\section{Fazit und Ausblick}

In der interdisziplinären Untersuchung zur Unfallgefährdung von Motorradfahrern konnten neue Erkenntnisse differenziert nach Ortslage sowohl aus Perspektive der Fahrzeugtechnik als auch der Straßenverkehrstechnik gewonnen und durch die Bildung typischer Unfallkonstellationen miteinander verknüpft werden. Den Schwerpunkt bildet das Unfallgeschehen auf Landstraßen und im Innerortsbereich. Motorradunfälle auf Autobahnen treten sehr selten auf.

Außerorts (ohne BAB) sind als straßenseitige Einflussfaktoren auf das Unfallgeschehen vor allem eine hohe Kurvigkeit sowie das Vorhandensein von Steigungs- und Gefälleabschnitten zu nennen. Knotenpunktdichte und Sichteinschränkungen besitzen ebenfalls einen Einfluss. Innerorts zeigen insbesondere Mängel im Straßenzustand sowie vorhandener Straßenbahnverkehr mit straßenbündiger Führung ungünstige Wirkung auf das Unfallgeschehen. Bei diesem Merkmal bleibt in zukünftigen Untersuchungen zu klären, ob das damit verbundene Sicherheitsproblem direkt mit der Eigenschaft des vorhandenen Bahnkörpers auf der Fahrbahn bzw. dem Straßenbahnverkehr verknüpft ist, oder in Verbindung mit anderen Eigenschaften steht, die im Zusammenhang mit diesem Merkmal gemeinsam auftreten.

Die Auswertungen bezogen auf die Fahrzeugtechnik ergaben einen statistisch signifikanten Einfluss des Leistungsgewichtes auf die Verletzungsschwere. Technische Eigenschaften wie z. B. das Vorhandensein eines Seitenkoffers am Einspurfahrzeug oder die Lenkerform haben hingegen keinen signifikanten Einfluss. Dasselbe gilt für den Motorradtyp als technisches Merkmal des Motorrades. Die häufigste Kollision bei Betrachtung der Unfallkonstellationen ist der aufrechte Anprall des Motorradfahrers mit stabiler Geradeausfahrt. Diese fällt jedoch im Mittel weniger schwer aus als der Sturz des Motorradfahrers vor der Kollision und der dann anschließenden Kollision. Alleinunfälle ohne Kollision mit einem Hindernis fallen i. d. R. weniger schwerwiegend aus als Unfälle mit Kollision.
Als wesentliches Ergebnis der Befragung von 6.879 Motorradfahrern resultiert eine besondere Auffälligkeit der Sportmaschinenfahrer. Diese geben an, besonders häufig in Ordnungswidrigkeitsdelikte verwickelt zu sein, wie z. B. zu hohe Geschwindigkeit oder falsche Überholmanöver. Zugleich verstehen sie sich als sportliche Fahrer. Das lässt darauf schließen, dass sie gerade die Fortbewegung in einem weniger kalkulierbaren Risikolevel suchen. Der Zusammenhang mit den Ergebnissen aus der GDV-Datenanalyse zeigt sich dadurch, dass Sportmotorräder mit einem besonders niedrigen Leistungsgewicht, bei denen das Beschleunigungsvermögen hoch ist, überproportional schwer in Unfälle verwickelt sind.

Die Rückkopplung zu den Ergebnissen der Straßenverkehrstechnik ergibt sich insbesondere für den Außerortsbereich. Strecken mit hoher Kurvigkeit und erheblichen Längsneigungen stellen eine besondere Attraktivität für die Motorradfahrer mit einer hohen Risikobereitschaft sowie Maschinen mit entsprechend hohem Beschleunigungsvermögen dar. Gerade diese Streckenabschnitte besitzen häufig eine hohe Unfalldichte. Außerorts passieren zudem die Hälfte aller Unfälle mit Beteiligung von motorisierten Zweirädern in Kurven und 1/3 aller Unfälle auf Steigungs- oder Gefällestrecken.

Aus der Bildung typischer Unfallkonstellationen auf Basis von Ortslage, Örtlichkeit, Unfalltyp und Unfallart resultierten konstellationsfeine Aussagen zum Unfallgeschehen (z. B. Unfallschwere, Hauptverursacher, Wochenendeinfluss). Für einen Teil der Konstellationen konnten ein typischer Unfallhergang, auffällige Zielgruppen sowie der Einfluss von Straßen- und Fahrzeugeigenschaften ermittelt werden. Auf dieser Grundlage wurden auf die einzelnen Unfallkonstellationen ausgerichtete Maßnahmen abgeleitet. Aussagekräftige Kollektive konnten vor allem im Innerortsbereich gebildet werden.

Als wesentliches Ergebnis für die untersuchten Zielgruppen ist festzuhalten, dass auf Landstraßen vor allem die risikoorientierten Sportfahrer im Freizeitbereich häufig 
bei Unfällen beteiligt sind. Dagegen weist die Altersgruppe, welche die Gruppe der Wiedereinsteiger beinhaltet, wenig Auffälligkeiten auf. Dies liegt aber auch an der Schwierigkeit, diese Gruppe eindeutig abzugrenzen. Danach wäre in zukünftigen Analysen zu suchen.

Innerorts ist die Aufmerksamkeit besonders auf jugendliche Fahrer und Fahranfänger zu richten. Jugendliche Fahrer unter 18 Jahren machen innerorts über 1/4 aller beteiligten Fahrer von PTW aus. In einer zukünftigen Untersuchung sollte auch die Gruppe der Rollerfahrer eingehender untersucht werden als dies in diesem Projekt geschehen konnte. In den Daten für die straßenseitige Auswertung war ausschließlich eine Abgrenzung der Kraftrollerfahrer möglich. Für diese sind die Fallzahlen insgesamt sehr gering. Roller mit einem Hubraum unter oder gleich $50 \mathrm{~cm}^{3}$ sind damit nicht erfasst. Im innerörtlichen Unfallgeschehen ist ein häufiges Auftreten von diesen jedoch anzunehmen. Allein für die Kraftrollerfahrer zeigten sich bereits Auffälligkeiten in einzelnen Unfallkonstellationen innerorts, deren Zufälligkeit aufgrund der geringen Fallzahlen jedoch nicht ausgeschlossen werden konnte.

In einer Folgeuntersuchung könnten die angesprochenen sowie weitere offen gebliebene Fragen - wie z. B. der Einfluss der Knotenpunktdichte auf das Unfallgeschehen (vgl. Abbildung 5-15) - zielgerichtet auf Basis der in diesem Projekt gemachten Erfahrungen interdisziplinär analysiert werden. 


\section{Abbildungsverzeichnis}

\begin{tabular}{|c|c|c|}
\hline Abbildung 1-1 & Sportmaschine & Seite 14 \\
\hline Abbildung 1-2 & Naked Bike & Seite 14 \\
\hline Abbildung 1-3 & Chopper & Seite 14 \\
\hline Abbildung 1-4 & Oldtimer & Seite 14 \\
\hline Abbildung 1-5 & Enduro & Seite 14 \\
\hline Abbildung 1-6 & Roller & Seite 14 \\
\hline Abbildung 1-7 & Tourer & Seite 14 \\
\hline Abbildung 1-8 & Mofa & Seite 14 \\
\hline Abbildung 2-1 & Struktur des Projektes & Seite 17 \\
\hline Abbildung 2-2 & Verknüpfungsparameter zwischen Datenbasen der TU Dresden und TU Berlin & Seite 19 \\
\hline Abbildung 3-1 & $\begin{array}{l}\text { Bestandsabhängige Verunglücktenentwicklung differenziert nach Altersklassen } \\
\text { [Koch 2006] }\end{array}$ & Seite 22 \\
\hline Abbildung 3-2 & $\begin{array}{l}\text { Kombinierte Betrachtung von Längsneigung, Querneigung und Kurvenausrich- } \\
\text { tung in [Gerlach 2007] }\end{array}$ & Seite 24 \\
\hline Abbildung 3-3 & $\begin{array}{l}\text { Schätzfehler bei der Wahrnehmung } \\
\text { Quelle: [Grimm 1988] }\end{array}$ & Seite 31 \\
\hline Abbildung 4-1 & $\begin{array}{l}\text { Entwicklung des Fahrzeugbestandes von motorisierten Fahrzeugen im Vergleich } \\
\text { zu den Personenkraftwagen nach [BMVBS 2006] }\end{array}$ & Seite 33 \\
\hline Abbildung 4-2 & $\begin{array}{l}\text { Entwicklung der Fahrleistungen von motorisierten Fahrzeugen im Vergleich zu } \\
\text { den Personenkraftwagen nach [BMVBS 2006] }\end{array}$ & Seite 34 \\
\hline Abbildung 4-3 & $\begin{array}{l}\text { Getötetenentwicklung in Deutschland getrennt nach Art der Beteiligung für den } \\
\text { Zeitraum von } 1991 \text { bis } 2006 \text { nach [Destatis 2007] }\end{array}$ & Seite 35 \\
\hline Abbildung 4-4 & $\begin{array}{l}\text { Verunglücktenentwicklung in Deutschland getrennt nach der Art der Beteiligung } \\
\text { für den Zeitraum von } 1991 \text { bis } 2006 \text { nach [Destatis 2007] }\end{array}$ & Seite 36 \\
\hline Abbildung 4-5 & $\begin{array}{l}\text { Entwicklung der direkt angepassten Unfallschwere für Unfälle mit Personenscha- } \\
\text { den in Deutschland für motorisierte Zweiradbenutzer und alle übrigen Verkehrs- } \\
\text { teilnehmergruppen für den Zeitraum von } 1996 \text { bis } 2005\end{array}$ & Seite 37 \\
\hline Abbildung 4-6 & $\begin{array}{l}\text { Entwicklung der Getötetenstruktur nach Ortslage für getötete Motorradfahrer in } \\
\text { Deutschland } 1991 \text { bis } 2005\end{array}$ & Seite 38 \\
\hline Abbildung 4-7 & $\begin{array}{l}\text { Entwicklung der Verunglücktenstruktur nach Ortslage für verunglückte Motor- } \\
\text { radfahrer in Deutschland } 1991 \text { bis } 2005\end{array}$ & Seite 39 \\
\hline Abbildung 4-8 & $\begin{array}{l}\text { Entwicklung der Getötetenrate der Nutzer von Motorraädern und Mopeds/Mofas } \\
\text { im Vergleich zu den Nutzern der übrigen Kraftfahrzeuge nach [BMVBS 2006] und } \\
\text { [Destatis 2007] }\end{array}$ & Seite 39 \\
\hline Abbildung 4-9 & $\begin{array}{l}\text { Entwicklung des Verhältnisses der Getötetenrate von Motorrädern bzw. Mopeds/ } \\
\text { Mofas zu den übrigen Kraftfahrzeugen von } 1991 \text { bis } 2005 \text { nach [Destatis 2007] } \\
\text { und [BMVBS 2006] }\end{array}$ & Seite 40 \\
\hline Abbildung 4-10 & $\begin{array}{l}\text { Aufteilung der U (P) der Zielgruppen nach Ortslage - Bundesstatistik } 2004 \text { und } \\
2005\end{array}$ & Seite 42 \\
\hline
\end{tabular}




\begin{tabular}{|c|c|c|}
\hline Abbildung 4-11 & $\begin{array}{l}\text { Relative Veränderung der Unfallhäufigkeit von } 2004 \text { und } 2005 \text { für die Zielgruppen } \\
\text { differenziert nach Ortslage }\end{array}$ & Seite 43 \\
\hline Abbildung 5-1 & $\begin{array}{l}\text { Getötetenbelastung nach Bundesländern für die Unfälle mit Motorradbeteili- } \\
\text { gung } 2003 \text { und } 2004\end{array}$ & Seite 44 \\
\hline Abbildung 5-2 & $\begin{array}{l}\text { Verunglücktenbelastung nach Bundesländern für die Unfälle mit Motorradbetei- } \\
\text { ligung } 2003 \text { und } 2004\end{array}$ & Seite 45 \\
\hline Abbildung 5-3 & $\begin{array}{l}\text { Fahrzeugbestandsbelastung nach Bundesländern für Motorräder } 2003 \text { und } \\
2004\end{array}$ & Seite 45 \\
\hline Abbildung 5-4 & $\begin{array}{l}\text { Vergleich der Unfallschwereverteilung in Sachsen und Deutschland für das Jahr } \\
2005 \text { - U (P+SS i.e.S.) mit Beteiligung motorisierter Zweiräder }\end{array}$ & Seite 46 \\
\hline Abbildung 5-5 & Vorgehen bei der Auswertung von Daten der besichtigten Motorradstrecken & Seite 50 \\
\hline Abbildung 5-6 & $\begin{array}{l}\text { Unfallschwere der Motorradunfälle nach Straßenzustand, an Knotenpunkten } \\
\text { ohne und mit Lichtsignalanlagen sowie in Kurven }\end{array}$ & Seite 51 \\
\hline Abbildung 5-7 & $\begin{array}{l}\text { Unfallschwere der Motorradunfälle auf Straßen mit Längsneigung nach Lichtver- } \\
\text { hältnissen }\end{array}$ & Seite 52 \\
\hline Abbildung 5-8 & Unfallschwere der Motorradunfälle nach Unfalltyp & Seite 53 \\
\hline Abbildung 5-9 & $\begin{array}{l}\text { Paarweiser Vergleich der Anteile der Abschnittslängen mit bestimmten Straßen- } \\
\text { eigenschaften außerorts (1) }\end{array}$ & Seite 59 \\
\hline Abbildung 5-10 & $\begin{array}{l}\text { Paarweiser Vergleich der Anteile der Abschnittslängen mit bestimmten Straßen- } \\
\text { eigenschaften außerorts ( } 2 \text { ) }\end{array}$ & Seite 60 \\
\hline g 5-11 & $\begin{array}{l}\text { Anteile der Unfälle an bestimmten Örtlichkeiten für die besichtigten Streckenab- } \\
\text { schnitte }\end{array}$ & Seite 61 \\
\hline Abbildung 5-12 & $\begin{array}{l}\text { Zusammenhang Unfalldichte und Knotendichte auf den besichtigen Strecken au- } \\
\text { ßerorts }\end{array}$ & Seite 62 \\
\hline Abbildung 5-13 & $\begin{array}{l}\text { Paarweiser Vergleich der Anteile der Abschnittslängen mit bestimmten Straßen- } \\
\text { eigenschaften innerorts (1) }\end{array}$ & Seite 63 \\
\hline Abbildung 5-14 & $\begin{array}{l}\text { Paarweiser Vergleich der Anteile der Abschnittslängen mit bestimmten Straßen- } \\
\text { eigenschaften innerorts ( } 2 \text { ) }\end{array}$ & Seite 64 \\
\hline Abbildung 5-15 & $\begin{array}{l}\text { Zusammenhang zwischen Unfalldichte und Knotendichte auf den besichtigten } \\
\text { Abschnitten innerorts }\end{array}$ & Seite 65 \\
\hline Abbildung 5-16 & $\begin{array}{l}\text { Verteilung der Unfälle auf den besichtigten Innerortsabschnitten nach Charakte- } \\
\text { ristik der Unfallstelle und Unfalltyp }\end{array}$ & Seite 66 \\
\hline Abbildung 5-17 & $\begin{array}{l}\text { Verteilung der Unfälle auf den besichtigten Außerortsabschnitten nach Charak- } \\
\text { teristik der Unfallstelle und Unfalltyp }\end{array}$ & Seite 67 \\
\hline Abbildung 5-18 & Typischer Unfallhergang der 10 definierten Unfallkonstellationen & Seite 68 \\
\hline Abbildung 6-1 & Zahl der Unfälle igo nach Leistungsgewicht und Charakteristik der Unfallstelle & Seite 69 \\
\hline Abbildung 6-2 & Zahl der Unfälle ago nach Leistungsgewicht und Charakteristik der Unfallstelle & Seite 70 \\
\hline Abbildung 6-3 & Anteil der Unfallschweren innerorts nach Leistungsgewicht & Seite 71 \\
\hline Abbildung 6-4 & Anteil der Unfallschweren außerorts nach Leistungsgewicht & Seite 72 \\
\hline Abbildung 6-5 & MAIS Verteilungüber die gewählten Unfallkonstellationen & Seite 73 \\
\hline Abbildung 6-6 & Verteilung der Verletzungsschwere & Seite 74 \\
\hline Abbildung 6-7 & Hauptanstoß bei Unverschulden & Seite 75 \\
\hline
\end{tabular}




\begin{tabular}{|c|c|c|}
\hline Abbildung 6-8 & MAIS Vergleich mit und ohne Kollision & Seite 75 \\
\hline Abbildung 6-9 & Alkohol und hohe Geschwindigkeit & Seite 76 \\
\hline Abbildung 6-10 & Auf die Motorradtypenhäufigkeit bezogenes Unfallrisiko & Seite 77 \\
\hline Abbildung 6-11 & MAIS der Motorradtypen in den Unfallkonstellationen & Seite 77 \\
\hline Abbildung 6-12 & Mittlere MAIS-Werte der Leistungsgewichtklassen und Unfallkonstellationen & Seite 78 \\
\hline Abbildung 6-13 & Anzahl der Leistungsgewichtklassen in den Unfallkonstellationen & Seite 79 \\
\hline Abbildung 6-14 & Mittlere MAIS-Werte der gesamten Leistungsgwichtklassen & Seite 79 \\
\hline Abbildung 6-15 & Verletzungsschwere über Leistungsgewicht ago mit Hauptschuldigkeit PTW & Seite 80 \\
\hline Abbildung 6-16 & Mittlere MAIS Verteilung über die Wochentage & Seite 83 \\
\hline Abbildung 6-17 & $\begin{array}{l}\text { Mittlere MAIS Verteilung abhängig von der Verkehrsdichte nach Angabe des } \\
\text { Beteiligten }\end{array}$ & Seite 83 \\
\hline Abbildung 6-18 & Verletzungsschwere abhängig von der Reaktion vor dem Unfallereignis & Seite 84 \\
\hline Abbildung 6-19 & MAIS-Werte bei Sturz & Seite 84 \\
\hline Abbildung 6-20 & Unfallhergang exemplarisch am Beispiel EK-Unfall außerorts an Einmündungen & Seite 85 \\
\hline Abbildung 6-21 & Anteil der MAIS 3+ Verletzungen der jeweiligen Körperregionen pro Unfallablauf & Seite 86 \\
\hline Abbildung 6-22 & Verletzungsschwere bei Überflug & Seite 87 \\
\hline Abbildung 6-23 & Anteil der selbstverschuldeten Unfälle innerorts nach Leistungsgewicht & Seite 87 \\
\hline Abbildung 6-24 & Anteil der selbstverschuldeten Unfälle außerorts nach Leistungsgewicht & Seite 88 \\
\hline Abbildung 6-25 & $\begin{array}{l}\text { Anteil der selbstverschuldeten Unfälle mit Todesfolge außerorts nach Leistungs- } \\
\text { gewicht }\end{array}$ & Seite 89 \\
\hline Abbildung 6-26 & Altersverteilung der Teilnehmer an der Befragung & Seite 91 \\
\hline Abbildung 6-27 & Verteilung der Motorradtypen in der Umfrage & Seite 92 \\
\hline Abbildung 6-28 & Durchschnittliche Fahrzeugdaten über die einzelnen Motorradtypen & Seite 93 \\
\hline Abbildung 6-29 & Motivation zum Motorradfahren & Seite 93 \\
\hline Abbildung 6-30 & Differenzierung der Motivation zum Motorradfahren nach Motorradtyp & Seite 94 \\
\hline Abbildung 6-31 & Nutzungsverhalten der Motorradfahrer über die unterschiedlichen Motorradtypen & Seite 95 \\
\hline Abbildung 6-32 & Nutzungsverhalten bei schlechtem Wetter & Seite 95 \\
\hline Abbildung 6-33 & Nutzungsverhalten im Winter & Seite 96 \\
\hline Abbildung 6-34 & Selbstgeschätztes Fahrkönnen & Seite 97 \\
\hline Abbildung 6-35 & Selbstgeschätzter Fahrstil & Seite 97 \\
\hline Abbildung 6-36 & Straßennutzungsverhalten Chopper & Seite 98 \\
\hline Abbildung 6-37 & Straßennutzungsverhalten Enduro & Seite 98 \\
\hline Abbildung 6-38 & Straßennutzungsverhalten Naked Bikes & Seite 98 \\
\hline Abbildung 6-39 & Straßennutzungsverhalten Roller & Seite 98 \\
\hline Abbildung 6-40 & Straßennutzungsverhalten Sportmaschinen & Seite 98 \\
\hline Abbildung 6-41 & Straßennutzungsverhalten Tourer & Seite 98 \\
\hline Abbildung 6-42 & Verkehrsverstöße der Befragten über Motorradtypen & Seite 99 \\
\hline Abbildung 6-43 & Art der Verkehrsverstöße & Seite 100 \\
\hline Abbildung 6-44 & Art des Verstoßes über das Leistungsgewicht & Seite 100 \\
\hline Abbildung 6-45 & Trageverhalten sicherer Schutzkleidung beim Motorradfahren & Seite 101 \\
\hline
\end{tabular}




\begin{tabular}{|l|l|r|}
\hline Abbildung 6-46 & Tragefrequenz eines Jethelms & Seite 102 \\
\hline Abbildung 6-47 & Tragefrequenz einer Jeans & Seite 102 \\
\hline Abbildung 6-48 & Verteilung der fremdverschuldeten Unfälle nach Ortslage und Motorradtyp & Seite 103 \\
\hline Abbildung 6-49 & Verteilung der selbstverschuldeten Unfälle nach Ortslage undn Motorradtyp & Seite 104 \\
\hline Abbildung 6-50 & Alleinunfälle nach Ortslage und Motorradtyp & Seite 104 \\
\hline Abbildung 6-51 & Alleinunfälle nach Leistungsgewicht und Ortslage & Seite 105 \\
\hline Abbildung 7-1 & $\begin{array}{l}\text { Vergleich der Kollektivgrößen von TU Berlin und TU Dresden für die Unfallkonstel- } \\
\text { lation }\end{array}$ & Seite 107 \\
\hline Abbildung 7-2 & Typische Unfallsituation zur Unfallkonstellation (2) & Seite 110 \\
\hline Abbildung 7-3 & Typische Unfallsituation zur Unfallkonstellation (4-1) - Einmündungen & Seite 113 \\
\hline Abbildung 7-4 & Typische Unfallsituation zur Unfallkonstellation (5) & Seite 115 \\
\hline Abbildung 7-5 & Typische Unfallssituation zur Unfallkonstellation (6) & Seite 116 \\
\hline Abbildung 7-6 & Typische Unfallssituation zur Unfallkonstellation (7) & Seite 118 \\
\hline
\end{tabular}




\section{Tabellenverzeichnis}

\begin{tabular}{|c|c|c|}
\hline Tabelle 1-1 & $\begin{array}{l}\text { Einteilung der morisierten Zweiräder nach StVZO und Statistischem Bundesamt } \\
\text { (Destatis) }\end{array}$ & Seite 12 \\
\hline Tabelle 1-2 & Vergleich der Einteilungen von motorisierten Zweirädern & Seite 13 \\
\hline Tabelle 1-3 & Festlegung der Kriterien für die Bestimmung der Zielgruppen in EUSka & Seite 16 \\
\hline Tabelle 3-1 & Übersicht der vorhandenen Unfalluntersuchungen mit Motorradbeteiligung & Seite 20 \\
\hline Tabelle 4-1 & Kriterien der Sonderabfrage beim Statistischen Bundesamt für spezielle Zielgruppen & Seite 41 \\
\hline Tabelle 5-1 & Abschnitte in Sachsen differenziert nach Ortslage und Unfalldichte & Seite 47 \\
\hline Tabelle 5-2 & Unfallanzahl und Unfalldichte der festgelegten Abschnitte & Seite 47 \\
\hline Tabelle 5-3 & Beispiel einer Vierfeldertabelle & Seite 47 \\
\hline Tabelle 5-4 & Kenndaten der für die Ortsbesichtigung ausgewählten Streckenabschnitte & Seite 48 \\
\hline Tabelle 5-5 & $\begin{array}{l}\text { Vergleich der Anteile der Zielgruppen - U (P) mit PTW-Beteiligung vs. U (P) aller } \\
\text { Verkehrsteilnehmer (innerorts) }\end{array}$ & Seite 56 \\
\hline Tabelle 5-6 & $\begin{array}{l}\text { Vergleich der Anteile der Zielgruppen - U (P) mit PTW-Beteiligung vs. U (P) aller } \\
\text { Verkehrsteilnehmer (außerorts) }\end{array}$ & Seite 57 \\
\hline Tabelle 5-7 & $\begin{array}{l}\text { Vergleich der Unfallanteile außerorts mit „Steigung“ oder "Gefälle“ als Charakteristik } \\
\text { der Unfallstelle }\end{array}$ & Seite 62 \\
\hline Tabelle 5-8 & Vergleich der Unfallanteile außerorts mit „Kurve“ als Charakteristik der Unfallstelle & Seite 62 \\
\hline Tabelle 6-1 & Häufigkeit der Unfallkonstellationen in den Daten des GDV & Seite 72 \\
\hline Tabelle 6-2 & Einteilung der Leistungsgewichte in 10 gleichgroße Klassen & Seite 78 \\
\hline Tabelle 6-3 & Altersklassen & Seite 81 \\
\hline Tabelle 6-4 & Leistungsklassen & Seite 81 \\
\hline Tabelle 6-5 & Varianzanalyse der Verletzungsschwere als abhängige Variable & Seite 81 \\
\hline Tabelle 6-6 & Post Hoc Test nach Scheffé & Seite 82 \\
\hline Tabelle 6-7 & Korrelation zwischen Höhe des Lenkers und des Leistungsgewichts & Seite 89 \\
\hline Tabelle 7-1 & Übersicht zur Unfallkonstellation (1) & Seite 108 \\
\hline Tabelle 7-2 & Übersicht zur Unfallkonstellation (2) & Seite 110 \\
\hline Tabelle 7-3 & Übersicht zur Unfallkonstellation (3) & Seite 111 \\
\hline Tabelle 7-4 & Übersicht zur Unfallkonstellation (4) & Seite 112 \\
\hline Tabelle 7-5 & Übersicht zur Unfallkonstellation (5) & Seite 116 \\
\hline Tabelle 7-6 & Übersicht zur Unfallkonstellation (6) & Seite 117 \\
\hline Tabelle 7-7 & Übersicht zur Unfallkonstellation (7) & Seite 119 \\
\hline Tabelle 7-8 & Übersicht zur Unfallkonstellation (8) & Seite 120 \\
\hline Tabelle 7-9 & Übersicht zur Unfallkonstellation (9) & Seite 121 \\
\hline Tabelle 7-10 & Übersicht zur Unfallkonstellation (10) & Seite 122 \\
\hline
\end{tabular}


\begin{tabular}{|l|l}
\hline Tabelle 8-1 & Maßnahmenvorschläge zu den 10 Unfallkonstellationen
\end{tabular}

\section{Literaturverzeichnis}

\section{[ACEM 2006]}

Association de Constructeurs Européens de Motocycles G.E.I.E.: Guidelines for PTW-safer Road Design in Europe, Brüssel, 2006.

[ADAC 2006]

ADAC: Honda Gold Wing mit und ohne Airbag, ADAC Crashtestvergleich, 2006.

\section{[Allianz 2006]}

Pressemeldung der Allianz Versicherungs-AG, München, April 2006.

\section{[Appel 2002]}

Appel, $H_{\text {., }}$ et al.: Unfallforschung, Unfallmechanik und Unfallrekonstruktion, Verlag INFORMATION Ambs $\mathrm{GmbH}$, Kippenheim, 2002.

\section{[Assing 2002]}

Assing, K.: Schwerpunkte des Unfallgeschehens von Motorradfahrern, Berichte der Bundesanstalt für Straßenwesen, Mensch und Sicherheit, Heft M 137. Bergisch Gladbach, 2002.

[Bast 1994]

BASt: Bremssysteme von Motorrädern, Bast Info 9/2004.

[Bast 1996]

Präckel, J.: Bremsverhalten von Fahrern von Motorrädern mit und ohne ABS, Berichte der Bundesanstalt für StraBenwesen, Fahrzeugtechnik, Heft F 18, Bergisch Gladbach, 1996.

\section{[BMVBS 2006]}

Bundesministerium für Verkehr, Bau und Städtebau: Verkehr in Zahlen 2006/07, Deutscher VerkehrsVerlag GmbH, Hamburg, 2006.
[Destatis 2007]

Statistisches Bundesamt: Verkehr, Fachserie 8 Reihe 7, Verkehrsunfälle, Statistisches Bundesamt, Wiesbaden 2007. [Elliot 2003]

Elliot, M.A., et al.: Motorcycle safety: a scoping study, TRL Report TRL581, 2003.

[FGSV 2003]

Forschungsgesellschaft für Straßen- und Verkehrswesen: Merkblatt zur Auswertung von Straßenverkehrsunfällen, Teil 1: Führen und Auswerten von Unfalltypensteckkarten, FGSV-Verlag, Köln, 2003.

[FGSV 2005]

Forschungsgesellschaft für Straßen- und Verkehrswesen: Handbuch zur Bemessung von Straßenverkehrsanalgen 2001, Fassung 2005, FGSV-Verlag, Köln, 2005.

[FGSV 2007]

Forschungsgesellschaft für Straßen- und Verkehrswesen: Merkblatt zur Verbesserung der Verkehrssicherheit auf Motorradstrecken, FGSV-Verlag, Köln, 2007.

[Gerlach 2007]

Gerlach, J., Oderwald, K.: Schutzeinrichtungen am Fahrbahnrand kritischer Streckenabschnitte für Motorradfahrer, BASt-Bericht V 152, Bergisch-Gladbach 2007.

[Grimm 1988]

Grimm, H.G.: Wahrnehmungsbedingungen und sicheres Verhalten im Straßenverkehr: Situationsübergreifende Aspekte, Bundesanstalt für Straßenwesen, Bergisch Gladbach, 1988.

[Gwehenberger 2006]

Gwehenberger, J., et al.: Schwerstunfälle mit Motorrädern - Analyse der Unfallstruktur und der Wirksamkeit von ABS, VKU Verkehrsunfall und Fahrzeugtechnik, Januar 2006. 
[Hessen 2003]

Hessisches Landesamt für Straßen- und Verkehrswesen: Maßnahmen zur Erhöhung der Verkehrssicherheit auf Motorradstrecken, 2003.

[Koch 2006]

Koch, H., Kisler, W.: Entwicklung des Motorradunfallgeschehens - Schlussbericht. Verkehrstechnisches Institut der Deutschen Versicherer des GDV, SV-Projekt 3013-05, Berlin, 2006.

[Kramlich 2000]

Kramlich, T., Sporner, A.: Zusammenspiel von aktiver und passiver Sicherheit bei Motorradkollisionen, GDV Institut für Fahrzeugsicherheit München, Vortrag INTERMOT 2000.

[Kramlich 2002]

Kramlich, T.: Noch immer gefährliche Begegnungen. Die häufigsten Gefahrensituationen für Motorradfahrer und die resultierenden Verletzungen, 4. Internationale Motorradkonferenz München, 2002.

[Kuroe 2005]

Kuroe, T.; et al.: Exploratory Study of an Airbag Concept for a Large Touring Motorcycle, Further Research Second Report. Paper No. 05-0316, 2005.

\section{[Leutzbach 1988]}

Leutzbach, W., Papavasiliou, V.: Wahrnehmungsbedingungen und sicheres Verhalten im Straßenverkehr: Wahrnehmung in konkreten Verkehrssituationen, Bundesanstalt für Straßenwesen, Bergisch Gladbach, 1988.

[Maier 2000]

Meewes, V., Maier, R.: Motorradfahrer - die unschuldigen Opfer?, Zeitschrift für Verkehrssicherheit 46, 2000.

[Pucher 2004]

Pucher, H. : Grundlagen der Verbrennungskraftmaschinen, Stichwortskriptum zur Vorlesung, TU-Berlin, 2004.
[Rücker 2005]

Rücker, P.; Berg, F.A.: Der Motorradairbag - neueste Erkenntnisse aus Full-Scale-Tests nach ISO 13232, Verkehrsunfall und Fahrzeugtechnik Heft 5, Mai 2005.

[Schönebeck 2005]

Schönebeck, S.; et al.: Abschätzung möglicher Auswirkungen von Fahren mit Licht am Tag (Tagfahrleuchten/ Abblendlicht) in Deutschland, Bast Abschlussbericht, Bergisch Gladbach; Juli 2005.

[Sporner 2006]

Sporner, A.: Datenbank - Motorradunfälle (SV-4509-04), München 2006.

[Stoffregen 1995]

Stoffregen, J.: Motorradtechnik. Grundlagen und Konzepte von Motor, Antrieb und Fahrwerk, Braunschweig/ Wiesbaden 1995.

[TÜV 2006]

Pressemeldung des TÜV Nord von April 2006.

[Unger 2004]

Unger, M.: Verbesserung der Wahrnehmbarkeit motorisierter Zweiradfahrer im Straßenverkehr, Studienarbeit 04/04, Berlin, 2004.

[van de Sand 2001]

van de Sand, A.; et al.: Anforderungen an Rückspiegel von Krafträdern, Bast-Info 19/01, Bast-Bericht, Heft F36, 2001.

[Winkelbauer 2002]

Winkelbauer, M.; Vavryn, K.: Bremsverzögerungsmessung bei Motorradfahrern mit und ohne ABS, Kuratorium für Verkehrssicherheit, Wien, Dezember 2002.

Weitere Quellen:

www.burning-out.de (2007)

http://www.burning-out.de/news/print.shtml?Philips-Mo- 
toVision-20060522120019 - Internetseite vom 21.04.2007.

n-tv (2007)

Beitrag in der Sendung vom 21.04.2007

\section{Anhang}

\section{Verzeichnis der Anlagen:}

- Anlage 01: Sonderauswertung der Bundesstatistik 2004 und 2005 zu den Zielgruppen

- Anlage 02: Übersicht über die in Sachsen festgelegten Streckenabschnitte (219)

- Anlage 03: Beispiel des Aufnahmeprotokolls zur Besichtigung der Streckenabschnitte

- Anlage 04: Ergebnisse der Unfallauswertungen zu den festgelegten Streckenabschnitten (Vierfeldertafeln)

- Anlage 05: Unfalltypenverteilung der Zielgruppen (Basis Unfälle auf den festgelegten Streckenabschnitten in Sachsen)

- Anlage 06: Überblick über untersuchte Unfallkollektive nach Ortslage, Charakteristik der Unfallstelle und Unfalltyp

- Anlage 07: Vergleich der Kennwerte zu den Unfallkonstellationen auf Basis unterschiedlicher KollektivgröBen

- Anlage 08: Detailauswertungen zu den Unfallkonstellationen

- Anlage 09: Fragebogen zur Befragung von Motorradfahrern in der Zeitschrift „MOTORRAD“

- Anlage 10: Beschreibung der zehn Unfallarten

- Anlage 11: Unfallaufnahme in Abhängigkeit von Unfallschwere und Anzahl der Beteiligten 
Anlage 01: Sonderauswertung der Bundesstatistik 2004 und 2005 zu den Zielgruppen
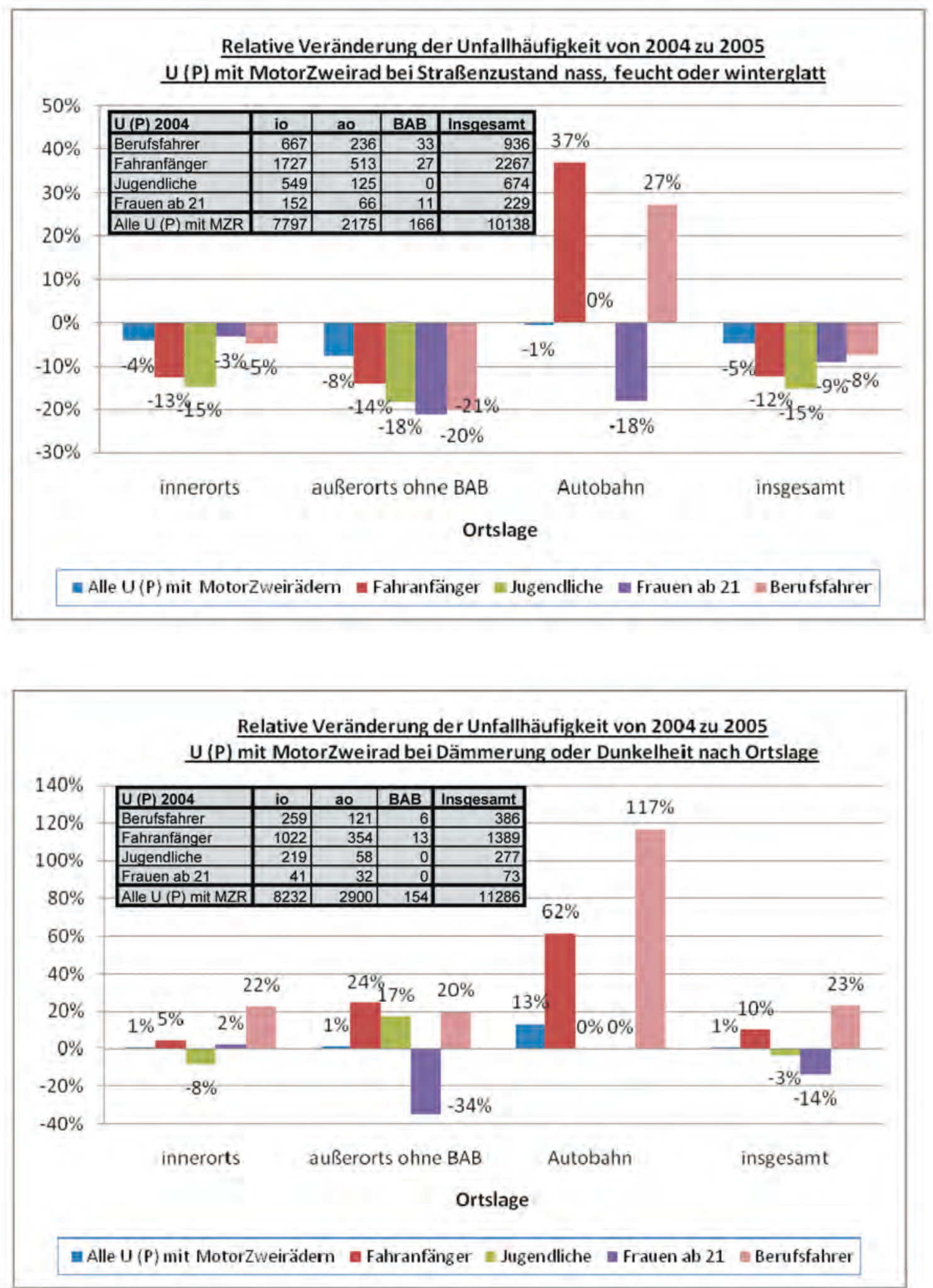

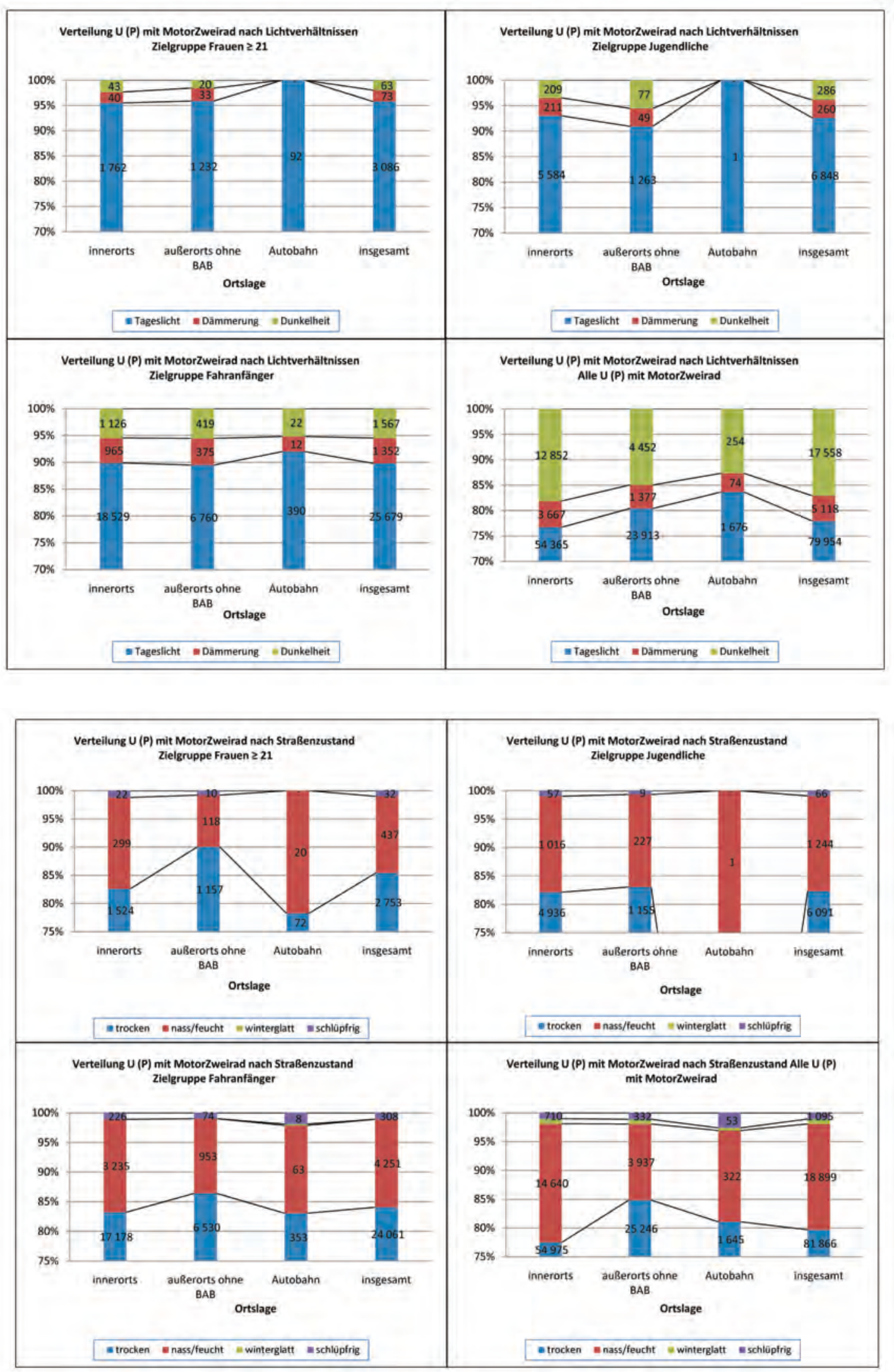
Anlage 02: Übersicht über die in Sachsen festgelegten Streckenabschnitte (219)

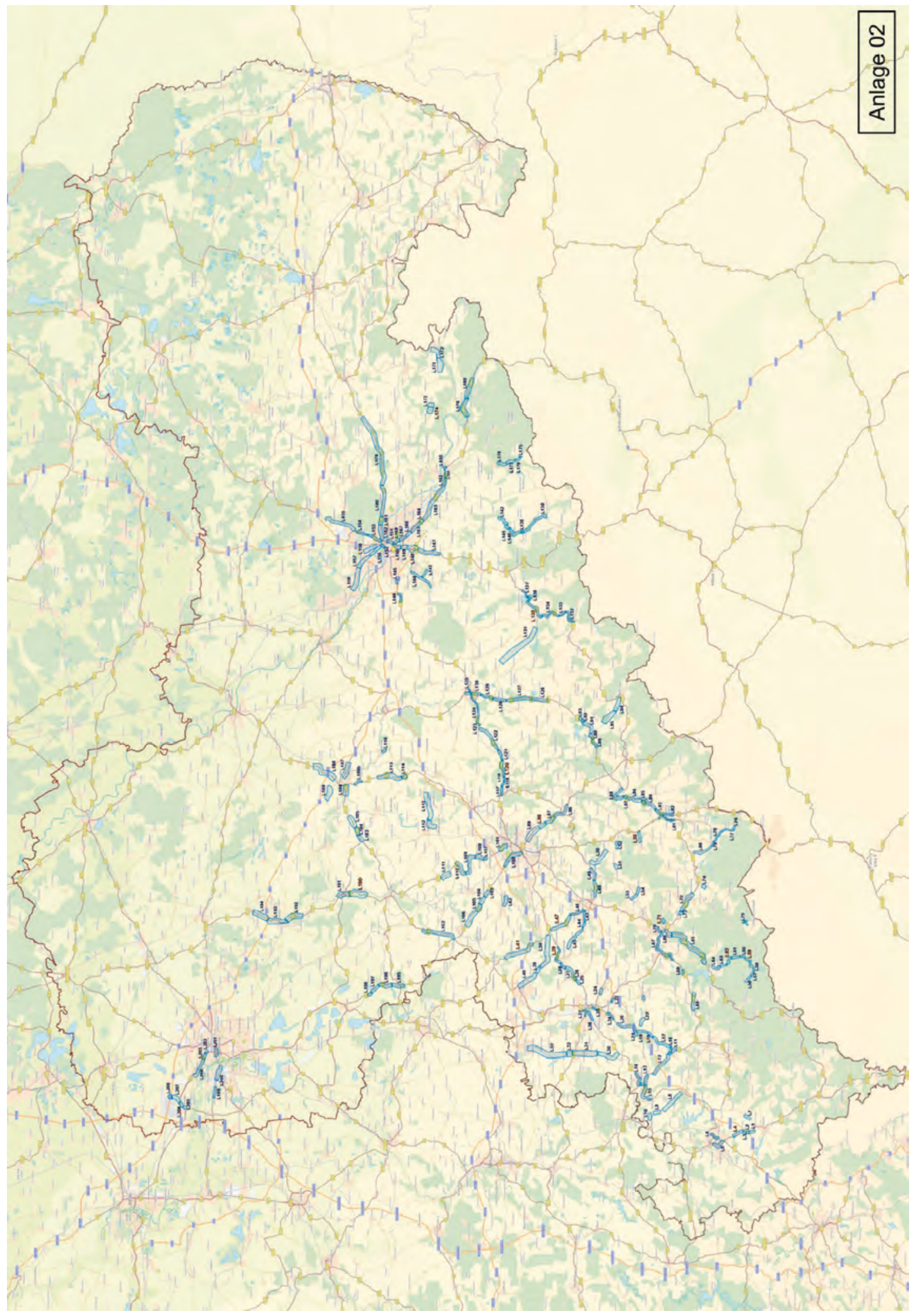


Anlage 03: Beispiel des Aufnahmeprotokolls zur Besichtigung der Streckenabschnitte

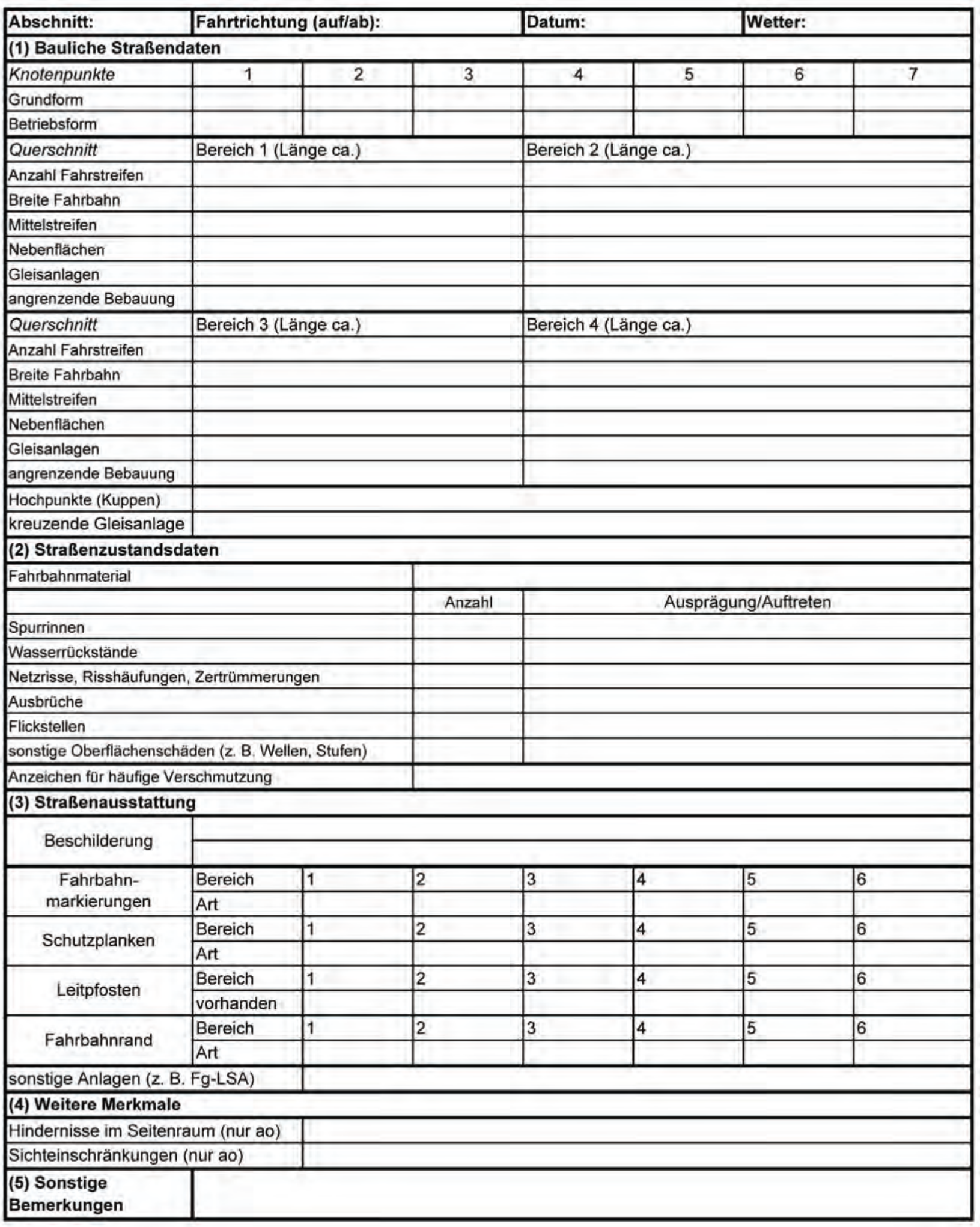


Anlage 04: Ergebnisse der Unfallauswertungen zu den festgelegten Streckenabschnitten (Vierfeldertafeln)

\begin{tabular}{|c|c|c|c|c|c|c|c|c|c|c|c|}
\hline \multicolumn{4}{|c|}{$\underline{U}(\mathrm{P}+\mathrm{S})$ mit Beteiligung motorisierter Zweiräder } & \multicolumn{4}{|c|}{$\underline{U}(P)$ mit Beteiligung motorisierter Zweiräder } & \multicolumn{4}{|c|}{$\underline{U}(P)$ alle Verkehrsteilnehmer } \\
\hline \multicolumn{4}{|c|}{ Anzahl Abschnitte } & \multicolumn{4}{|c|}{ Anzahl Abschnitte } & \multicolumn{4}{|c|}{ Anzahl Abschnitte } \\
\hline & geringe UD & hohe UD & Summe & & geringe UD & hohe UD & Summe & & geringe UD & hohe UD & Summe \\
\hline Innerorts & 70 & 46 & 116 & Innerorts & 70 & 46 & 116 & Innerorts & 70 & 46 & 116 \\
\hline Außerorts & 52 & 51 & 103 & Außerorts & 52 & 51 & 103 & Außerorts & 52 & 51 & 103 \\
\hline Summe & 122 & 97 & 219 & Summe & 122 & 97 & 219 & Summe & 122 & 97 & 219 \\
\hline \multicolumn{4}{|c|}{ mittlere Abschnittslänge in [km] } & \multicolumn{4}{|c|}{ mittlere Abschnittslänge in [km] } & \multicolumn{4}{|c|}{ mittlere Abschnittslänge in [km] } \\
\hline & geringe UD & hohe UD & alle & & geringe UD & hohe UD & alle & & geringe UD & hohe UD & alle \\
\hline Innerorts & 2,6 & 2,2 & 2,4 & Innerorts & 2,6 & 2,2 & 2,4 & Innerorts & 2,6 & 2,2 & 2,4 \\
\hline Außerorts & 3,5 & 2,2 & 2,9 & Außerorts & 3,5 & 2,2 & 2,9 & Außerorts & 3,5 & 2,2 & 2,9 \\
\hline alle & 3,0 & 2,2 & 2,6 & alle & 3,0 & 2,2 & 2,6 & alle & 3,0 & 2,2 & 2,6 \\
\hline \multicolumn{4}{|c|}{ Summierte Abschnittslängen in [m] } & \multicolumn{4}{|c|}{ Summierte Abschnittslängen in [m] } & \multicolumn{4}{|c|}{ Summierte Abschnittslängen in [m] } \\
\hline & geringe UD & hohe UD & Summe & & geringe UD & hohe UD & Summe & & geringe UD & hohe UD & Summe \\
\hline Innerorts & 180.520 & 100.146 & 280.666 & Innerorts & 180.520 & 100.146 & 280.666 & Innerorts & 180.520 & 100.146 & 280.666 \\
\hline Außerorts & 182.502 & 113.428 & 295.930 & Außerorts & 182.502 & 113.428 & 295.930 & Außerorts & 182.502 & 113.428 & 295.930 \\
\hline Summe & 363.022 & 213.574 & 576.596 & Summe & 363.022 & 213.574 & 576.596 & Summe & 363.022 & 213.574 & 576.596 \\
\hline \multicolumn{4}{|c|}{ Unfallanzahl 2004-2006 } & \multicolumn{4}{|c|}{ Unfallanzahl 2004-2006 } & \multicolumn{4}{|c|}{ Unfallanzahl 2004-2006 } \\
\hline & geringe UD & hohe UD & Summe & & geringe UD & hohe UD & Summe & & geringe UD & hohe UD & Summe \\
\hline Innerorts & 473 & 726 & 1199 & Innerorts & 290 & 402 & 692 & Innerorts & 1489 & 2336 & 3825 \\
\hline Außerorts & 151 & 272 & 423 & Außerorts & 121 & 209 & 330 & Außerorts & 566 & 630 & 1196 \\
\hline Summe & 624 & 998 & 1622 & Summe & 411 & 611 & 1022 & Summe & 2055 & 2966 & 5021 \\
\hline \multicolumn{4}{|c|}{ Unfalldichte in [U / $(\mathrm{km} \times$ a) $]$} & \multicolumn{4}{|c|}{ Unfalldichte in [U / $(\mathrm{km} \times \mathrm{a})]$} & \multicolumn{4}{|c|}{ Unfalldichte in [U / $(\mathrm{km} \times \mathrm{a})]$} \\
\hline & geringe UD & hohe UD & alle & & geringe UD & hohe UD & alle & & geringe UD & hohe UD & alle \\
\hline Innerorts & 0,9 & 2,4 & 1,4 & Innerorts & 0,5 & 1,3 & 0,8 & Innerorts & 2,7 & 7,8 & 4,5 \\
\hline Außerorts & 0,3 & 0,8 & 0,5 & Außerorts & 0,2 & 0,6 & 0,4 & Außerorts & 1,0 & 1,9 & 1,3 \\
\hline alle & 0,6 & 1,6 & 0,9 & alle & 0,4 & 1,0 & 0,6 & alle & 1,9 & 4,6 & 2,9 \\
\hline
\end{tabular}

\begin{tabular}{|c|c|c|c|c|c|c|c|c|c|c|c|}
\hline \multicolumn{4}{|c|}{ U (P+S) mit Beteiliqung motorisierter Zweiräder } & \multicolumn{4}{|c|}{$\underline{U}(P)$ mit Beteiligung motorisierter $Z$ weiräder } & \multicolumn{4}{|c|}{$\underline{U}(\mathrm{P})$ alle Verkehrsteilnehmer } \\
\hline \multicolumn{4}{|c|}{ Anteil U(GT) } & \multicolumn{4}{|c|}{ Anteil U(GT) } & \multicolumn{4}{|c|}{ Anteil U (GT) } \\
\hline & geringe UD & hohe UD & alle & & geringe UD & hohe UD & alle & & geringe UD & hohe UD & alle \\
\hline Innerorts & $1 \%$ & $0 \%$ & $1 \%$ & Innerorts & $2 \%$ & $1 \%$ & $1 \%$ & Innerorts & $1 \%$ & $1 \%$ & $1 \%$ \\
\hline Außerorts & $6 \%$ & $5 \%$ & $5 \%$ & Außerorts & $7 \%$ & $6 \%$ & $7 \%$ & Außerorts & $4 \%$ & $5 \%$ & $4 \%$ \\
\hline alle & $3 \%$ & $2 \%$ & $2 \%$ & alle & $4 \%$ & $3 \%$ & $3 \%$ & alle & $2 \%$ & $2 \%$ & $2 \%$ \\
\hline \multicolumn{4}{|c|}{ Anteil U(SP) } & \multicolumn{4}{|c|}{ Anteil U(SP) } & \multicolumn{4}{|c|}{ Anteil U (SP) } \\
\hline & geringe UD & hohe UD & alle & & geringe UD & hohe UD & alle & & geringe UD & hohe UD & alle \\
\hline Innerorts & $23 \%$ & $16 \%$ & $19 \%$ & Innerorts & $37 \%$ & $28 \%$ & $32 \%$ & Innerorts & $29 \%$ & $20 \%$ & $24 \%$ \\
\hline Außerorts & $54 \%$ & $46 \%$ & $48 \%$ & Außerorts & $67 \%$ & $59 \%$ & $62 \%$ & Außerorts & $44 \%$ & $43 \%$ & $44 \%$ \\
\hline alle & $30 \%$ & $24 \%$ & $26 \%$ & alle & $46 \%$ & $39 \%$ & $42 \%$ & alle & $33 \%$ & $25 \%$ & $28 \%$ \\
\hline \multicolumn{4}{|c|}{ Anteil U (P) } & \multicolumn{4}{|c|}{ Anteil U (P) } & \multicolumn{4}{|c|}{ Anteil U (P) } \\
\hline & geringe UD & hohe UD & alle & & geringe UD & hohe UD & alle & & geringe UD & hohe UD & alle \\
\hline Innerorts & $61 \%$ & $55 \%$ & $58 \%$ & Innerorts & & & & Innerorts & & & \\
\hline Außerorts & $80 \%$ & $77 \%$ & $78 \%$ & Außerorts & & & & Außerorts & & & \\
\hline alle & $66 \%$ & $61 \%$ & $63 \%$ & alle & & & & alle & & & \\
\hline \multicolumn{4}{|c|}{ Anteil Fahrunfall } & \multicolumn{4}{|c|}{ Anteil Fahrunfall } & \multicolumn{4}{|c|}{ Anteil Fahrunfall } \\
\hline & geringe UD & hohe UD & alle & & geringe UD & hohe UD & alle & & geringe UD & hohe UD & alle \\
\hline Innerorts & $14 \%$ & $11 \%$ & $12 \%$ & Innerorts & $19 \%$ & $14 \%$ & $16 \%$ & Innerorts & $16 \%$ & $8 \%$ & $11 \%$ \\
\hline Außerorts & $44 \%$ & $40 \%$ & $42 \%$ & Außerorts & $48 \%$ & $47 \%$ & $47 \%$ & Außerorts & $43 \%$ & $39 \%$ & $41 \%$ \\
\hline alle & $22 \%$ & $19 \%$ & $20 \%$ & alle & $28 \%$ & $25 \%$ & $26 \%$ & alle & $24 \%$ & $15 \%$ & $19 \%$ \\
\hline \multicolumn{4}{|c|}{ Anteil Abbiegeunfall } & \multicolumn{4}{|c|}{ Anteil Abbiegeunfall } & \multicolumn{4}{|c|}{ Anteil Abbiegeunfall } \\
\hline & geringe UD & hohe UD & alle & & geringe UD & hohe UD & alle & & geringe UD & hohe UD & alle \\
\hline Innerorts & $20 \%$ & $18 \%$ & $19 \%$ & Innerorts & $23 \%$ & $21 \%$ & $22 \%$ & Innerorts & $17 \%$ & $16 \%$ & $17 \%$ \\
\hline Außerorts & $15 \%$ & $15 \%$ & $15 \%$ & Außerorts & $12 \%$ & $14 \%$ & $13 \%$ & Außerorts & $10 \%$ & $15 \%$ & $13 \%$ \\
\hline alle & $19 \%$ & $17 \%$ & $18 \%$ & alle & $20 \%$ & $18 \%$ & $19 \%$ & alle & $15 \%$ & $16 \%$ & $16 \%$ \\
\hline \multicolumn{4}{|c|}{ Anteil Einbiegen/Kreuzen-Unfall } & \multicolumn{4}{|c|}{ Anteil Einbiegen/Kreuzen-Unfall } & \multicolumn{4}{|c|}{ Anteil Einbiegen/Kreuzen-Unfall } \\
\hline & geringe UD & hohe UD & alle & & geringe UD & hohe UD & alle & & geringe UD & hohe UD & alle \\
\hline Innerorts & $27 \%$ & $25 \%$ & $26 \%$ & Innerorts & $32 \%$ & $32 \%$ & $32 \%$ & Innerorts & $27 \%$ & $28 \%$ & $28 \%$ \\
\hline Außerorts & $12 \%$ & $15 \%$ & $14 \%$ & Außerorts & $12 \%$ & $14 \%$ & $14 \%$ & Außerorts & $12 \%$ & $14 \%$ & $13 \%$ \\
\hline alle & $23 \%$ & $22 \%$ & $23 \%$ & alle & $26 \%$ & $26 \%$ & $26 \%$ & alle & $23 \%$ & $25 \%$ & $24 \%$ \\
\hline
\end{tabular}




\begin{tabular}{|c|c|c|c|c|c|c|c|c|c|c|c|}
\hline \multicolumn{4}{|c|}{$\underline{U}(\mathrm{P}+\mathrm{S})$ mit Beteiligung motorisierter Zweiräder } & \multicolumn{4}{|c|}{$\underline{U}(\mathrm{P})$ mit Beteiligung motorisierter Zweiräder } & \multicolumn{4}{|c|}{$\underline{U}(P)$ alle Verkehrsteilnehmer } \\
\hline \multicolumn{4}{|c|}{ Anteil Unfall im Längsverkehr } & \multicolumn{4}{|c|}{ Anteil Unfall im Längsverkehr } & \multicolumn{4}{|c|}{ Anteil Unfall im Längsverkehr } \\
\hline & geringe UD & hohe UD & alle & & geringe UD & hohe UD & alle & & geringe UD & hohe UD & alle \\
\hline Innerorts & $32 \%$ & $37 \%$ & $35 \%$ & Innerorts & $17 \%$ & $22 \%$ & $20 \%$ & Innerorts & $27 \%$ & $29 \%$ & $28 \%$ \\
\hline Außerorts & $19 \%$ & $22 \%$ & $21 \%$ & Außerorts & $17 \%$ & $18 \%$ & $17 \%$ & Außerorts & $26 \%$ & $25 \%$ & $25 \%$ \\
\hline alle & $29 \%$ & $33 \%$ & $31 \%$ & alle & $17 \%$ & $20 \%$ & $19 \%$ & alle & $26 \%$ & $28 \%$ & $27 \%$ \\
\hline \multicolumn{4}{|c|}{ Anteil Unfälle am Wochendende (Sa/So) } & \multicolumn{4}{|c|}{ Anteil Unfalle am Wochendende (Sa/So) } & \multicolumn{4}{|c|}{ Anteil Unfälle am Wochendende (Sa/So) } \\
\hline & geringe UD & hohe UD & alle & & geringe UD & hohe UD & alle & & geringe UD & hohe UD & alle \\
\hline Innerorts & $25 \%$ & $16 \%$ & $19 \%$ & Innerorts & $26 \%$ & $14 \%$ & $19 \%$ & Innerorts & $22 \%$ & $18 \%$ & $20 \%$ \\
\hline Außerorts & $41 \%$ & $37 \%$ & $38 \%$ & Außerorts & $42 \%$ & $38 \%$ & $39 \%$ & Außerorts & $33 \%$ & $32 \%$ & $32 \%$ \\
\hline alle & $29 \%$ & $22 \%$ & $24 \%$ & alle & $31 \%$ & $22 \%$ & $25 \%$ & alle & $25 \%$ & $21 \%$ & $23 \%$ \\
\hline \multicolumn{4}{|c|}{ Anteil Unfälle bei Schlechtwetterbedingungen" } & \multicolumn{4}{|c|}{ Anteil Unfälle bei Schlechtwetterbedingungen } & \multicolumn{4}{|c|}{ Anteil Unfälle bei Schlechtwetterbedingungen } \\
\hline & geringe UD & hohe UD & alle & & geringe UD & hohe UD & alle & & geringe UD & hohe UD & alle \\
\hline Innerorts & $28 \%$ & $33 \%$ & $31 \%$ & Innerorts & $17 \%$ & $20 \%$ & $19 \%$ & Innerorts & $31 \%$ & $27 \%$ & $29 \%$ \\
\hline Außerorts & $14 \%$ & $22 \%$ & $19 \%$ & Außerorts & $9 \%$ & $15 \%$ & $13 \%$ & Außerorts & $39 \%$ & $37 \%$ & $38 \%$ \\
\hline alle & $24 \%$ & $29 \%$ & $27 \%$ & alle & $14 \%$ & $18 \%$ & $17 \%$ & alle & $33 \%$ & $29 \%$ & $31 \%$ \\
\hline \multicolumn{12}{|c|}{ Strabenzustand nass oder Winterglatt nach VUA } \\
\hline \multicolumn{4}{|c|}{ Anteil Unfälle Monate November-März } & \multicolumn{4}{|c|}{ Anteil Unfälle Monate November-März } & \multicolumn{4}{|c|}{ Anteil Unfälle Monate November-März } \\
\hline & geringe UD & hohe UD & alle & & geringe UD & hohe UD & alle & & geringe UD & hohe UD & alle \\
\hline Innerorts & $23 \%$ & $26 \%$ & $25 \%$ & Innerorts & $13 \%$ & $13 \%$ & $13 \%$ & Innerorts & $33 \%$ & $32 \%$ & $32 \%$ \\
\hline Außerorts & $9 \%$ & $12 \%$ & $11 \%$ & Außerorts & $6 \%$ & $7 \%$ & $6 \%$ & Außerorts & $33 \%$ & $30 \%$ & $31 \%$ \\
\hline alle & $19 \%$ & $21 \%$ & $20 \%$ & alle & $11 \%$ & $11 \%$ & $11 \%$ & alle & $33 \%$ & $31 \%$ & $32 \%$ \\
\hline \multicolumn{4}{|c|}{ Anteil Unfälle bei Dämmerung/Dunkelheit } & \multicolumn{4}{|c|}{ Anteil Unfälle bei Dåmmerung/Dunkelheit } & \multicolumn{4}{|c|}{ Anteil Unfälle bei Dåmmerung/Dunkelheit } \\
\hline & geringe UD & hohe UD & alle & & geringe UD & hohe UD & alle & & geringe UD & hohe UD & alle \\
\hline Innerorts & $21 \%$ & $18 \%$ & $19 \%$ & Innerorts & $23 \%$ & $20 \%$ & $22 \%$ & Innerorts & $23 \%$ & $26 \%$ & $25 \%$ \\
\hline Außerorts & $13 \%$ & $15 \%$ & $14 \%$ & Außerorts & $13 \%$ & $15 \%$ & $15 \%$ & Außerorts & $30 \%$ & $25 \%$ & $27 \%$ \\
\hline alle & $19 \%$ & $17 \%$ & $18 \%$ & alle & $20 \%$ & $19 \%$ & $19 \%$ & alle & $25 \%$ & $26 \%$ & $26 \%$ \\
\hline
\end{tabular}
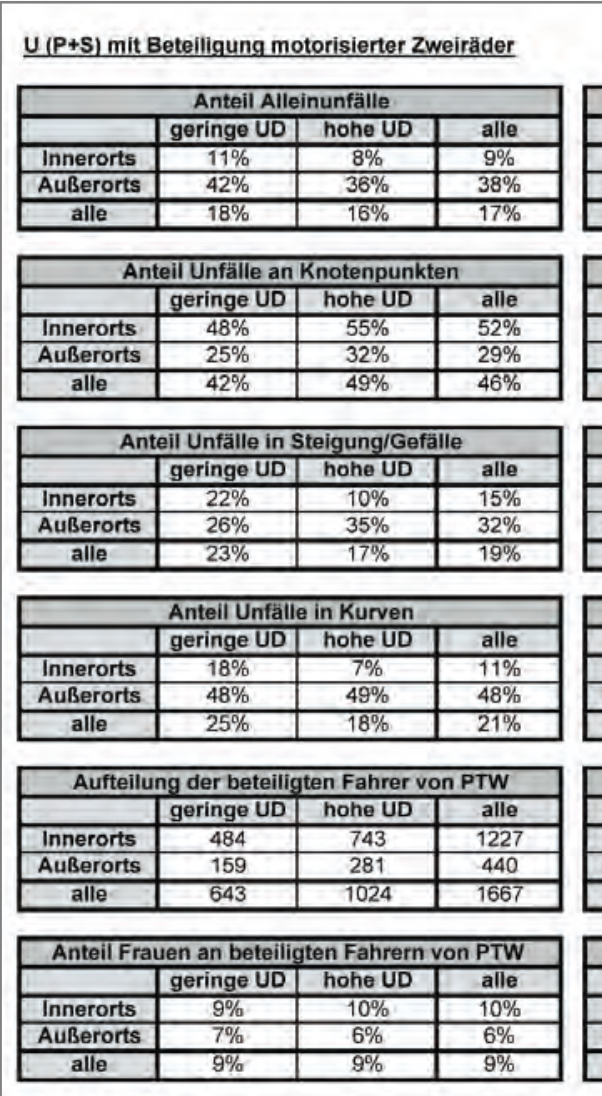

$\underline{U}$ (P) mit Beteiligung motorisierter Zweiräder
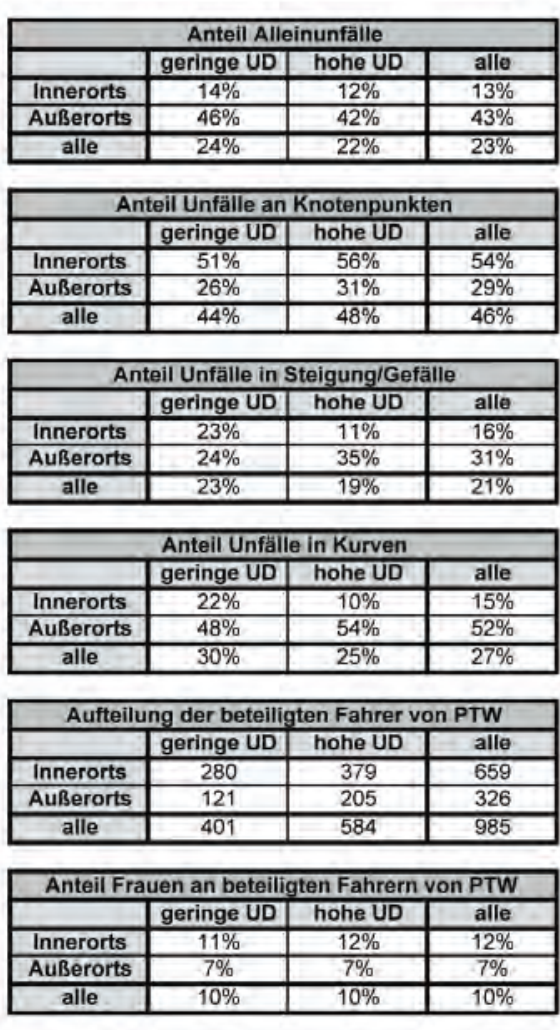

$\underline{\text { U (P) alle Verkehrsteilnehmer }}$
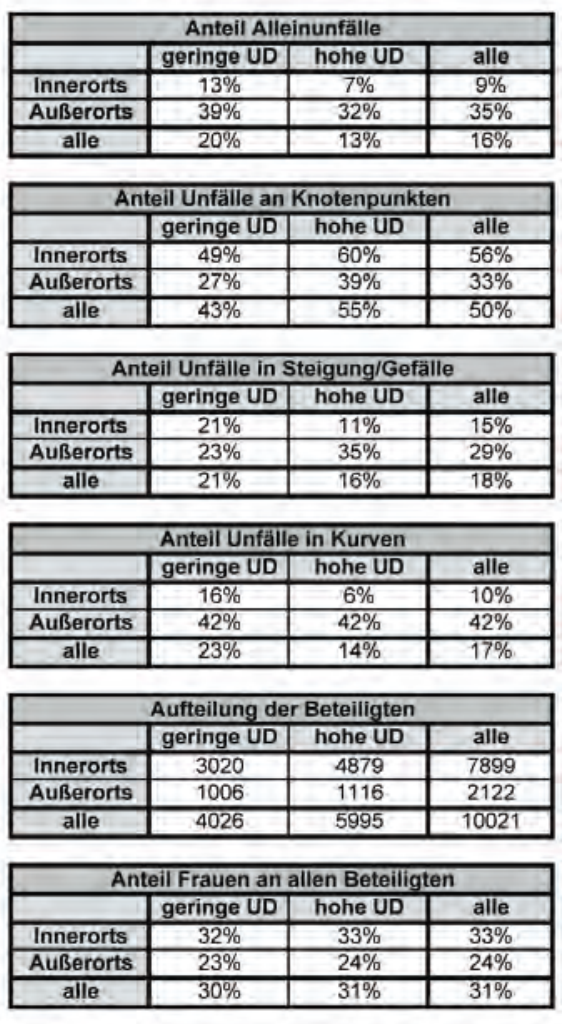


\begin{tabular}{|c|c|c|c|c|c|c|c|c|c|c|c|}
\hline \multicolumn{4}{|c|}{$\underline{U}(\mathrm{P}+\mathrm{S})$ mit Beteiliqung motorisierter Zweirăder } & \multicolumn{4}{|c|}{$\underline{U}(\mathrm{P})$ mit Beteiligung motorisierter Zweirăder } & \multicolumn{4}{|c|}{$\underline{U(P) \text { alle Verkehrsteilnehmer }}$} \\
\hline \multicolumn{4}{|c|}{ Anteil Jugendliche an beteiligten PTW-Fahrern } & \multicolumn{4}{|c|}{ Anteil Jugendliche an beteiligten PTW-Fahrern } & \multicolumn{4}{|c|}{ Anteil Jugendliche an allen Beteiligten } \\
\hline & \begin{tabular}{|l|} 
geringe UD \\
\end{tabular} & hohe UD & alle & & geringe UD & hohe UD & alle & & geringe UD & hohe UD & alle \\
\hline \begin{tabular}{|l|} 
Innerorts \\
\end{tabular} & $34 \%$ & $21 \%$ & $26 \%$ & Innerorts & $33 \%$ & $18 \%$ & $24 \%$ & Innerorts & $8 \%$ & $5 \%$ & $6 \%$ \\
\hline \begin{tabular}{|l|} 
Außerorts \\
\end{tabular} & $15 \%$ & $16 \%$ & $15 \%$ & Außerorts & $15 \%$ & $12 \%$ & $13 \%$ & Außerorts & $3 \%$ & $3 \%$ & $3 \%$ \\
\hline alle & $29 \%$ & $20 \%$ & $23 \%$ & alle & $27 \%$ & $16 \%$ & $21 \%$ & alle & $7 \%$ & $5 \%$ & $5 \%$ \\
\hline \multicolumn{12}{|l|}{ <18 Jahre } \\
\hline \multicolumn{4}{|c|}{ Anteil Fahranfanger an beteiligten PTW-Fahrern } & \multicolumn{4}{|c|}{ Anteil Fahranfänger an beteiligten PTW-Fahrern } & \multicolumn{4}{|c|}{ Anteil Beteiligte mit Fürerscheinalter $<=2$ Jahre } \\
\hline & geringe UD & hohe UD & alle & & geringe UD & hohe UD & alle & & geringe UD & hohe UD & alle \\
\hline \begin{tabular}{|l|} 
Innerorts \\
\end{tabular} & $21 \%$ & $20 \%$ & $20 \%$ & Innerorts & $21 \%$ & $22 \%$ & $22 \%$ & Innerorts & $9 \%$ & $9 \%$ & $9 \%$ \\
\hline \begin{tabular}{|l|} 
Außerorts \\
\end{tabular} & $15 \%$ & $13 \%$ & $14 \%$ & \begin{tabular}{|l} 
Außerorts \\
\end{tabular} & $16 \%$ & $14 \%$ & $15 \%$ & Außerorts & $13 \%$ & $11 \%$ & $12 \%$ \\
\hline alle & $20 \%$ & $18 \%$ & $19 \%$ & alle & $19 \%$ & $19 \%$ & $19 \%$ & alle & $10 \%$ & $10 \%$ & $10 \%$ \\
\hline \multicolumn{4}{|c|}{ Anteil Senioren an beteiligten Fahrern von PTW } & \multicolumn{4}{|c|}{ Anteil Senioren an beteiligten Fahrern von PTW } & \multicolumn{4}{|c|}{ Anteil Senioren an allen Beteiligten } \\
\hline & \begin{tabular}{|l|} 
geringe UD \\
\end{tabular} & hohe UD & alle & & \begin{tabular}{|l|l|} 
geringe UD \\
\end{tabular} & hohe UD & alle & & geringe UD & hohe UD & alle \\
\hline Innerorts & $5 \%$ & $6 \%$ & $6 \%$ & Innerorts & $6 \%$ & $7 \%$ & $7 \%$ & Innerorts & $20 \%$ & $16 \%$ & $17 \%$ \\
\hline \begin{tabular}{|l|} 
Außerorts \\
\end{tabular} & $8 \%$ & $8 \%$ & $8 \%$ & Außerorts & $10 \%$ & $9 \%$ & $10 \%$ & Außerorts & $15 \%$ & $16 \%$ & $16 \%$ \\
\hline alle & $6 \%$ & $7 \%$ & $6 \%$ & alle & $7 \%$ & $8 \%$ & $8 \%$ & alle & $19 \%$ & $16 \%$ & $17 \%$ \\
\hline \multicolumn{12}{|l|}{$y=55$ Jahre } \\
\hline \multicolumn{4}{|c|}{ Anteil Kraftrollerfahrer an bet. PTW-Fahrern } & \multicolumn{4}{|c|}{ Anteil Kraftrollerfahrer an bet. PTW-Fahrern } & & & & \\
\hline & geringe UD & hohe UD & alle & & geringe UD & hohe UD & alle & & & & \\
\hline Innerorts & $1 \%$ & $4 \%$ & $3 \%$ & Innerorts & $1 \%$ & $4 \%$ & $3 \%$ & & & & \\
\hline \begin{tabular}{|l|} 
Außerorts \\
\end{tabular} & $1 \%$ & $1 \%$ & $1 \%$ & Außerorts & $2 \%$ & $0 \%$ & $1 \%$ & & & & \\
\hline alle & $1 \%$ & $3 \%$ & $2 \%$ & alle & $1 \%$ & $3 \%$ & $2 \%$ & & & & \\
\hline \multicolumn{4}{|c|}{ Anteil U mit PTW-Fahrer als Hauptverursacher } & \multicolumn{4}{|c|}{ Anteil U (P) mit PTW-Fahrer als Hauptverursacher } & & & & \\
\hline & geringe UD & hohe UD & alle & & geringe UD & hohe UD & alle & & & & \\
\hline Innerorts & $52 \%$ & $48 \%$ & $50 \%$ & Innerorts & $43 \%$ & $40 \%$ & $41 \%$ & & & & \\
\hline Außerorts & $74 \%$ & $66 \%$ & $69 \%$ & Außerorts & $74 \%$ & $66 \%$ & $69 \%$ & & & & \\
\hline alle & $57 \%$ & $53 \%$ & $55 \%$ & alle & $52 \%$ & $49 \%$ & $50 \%$ & & & & \\
\hline
\end{tabular}


Anlage 05: Unfalltypenverteilung der Zielgruppen (Basis Unfälle auf den festgelegten Streckenabschnitten in Sachsen)

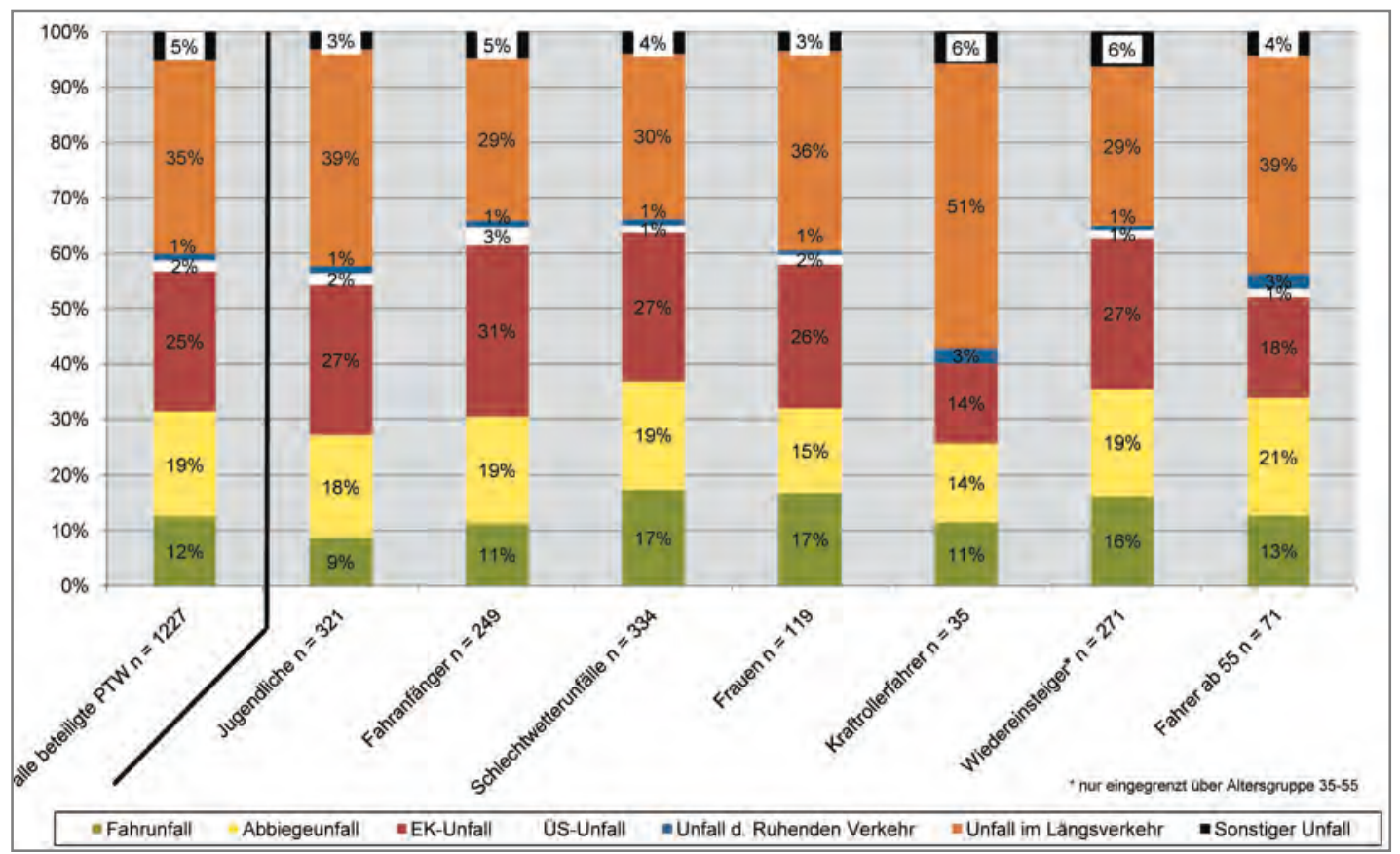

Unfalltypenverteilung der Zielgruppen für alle motorsierten Zweiradfahrer bei Innerortsunfällen auf den festgelegten Streckenabschnitten in Sachsen

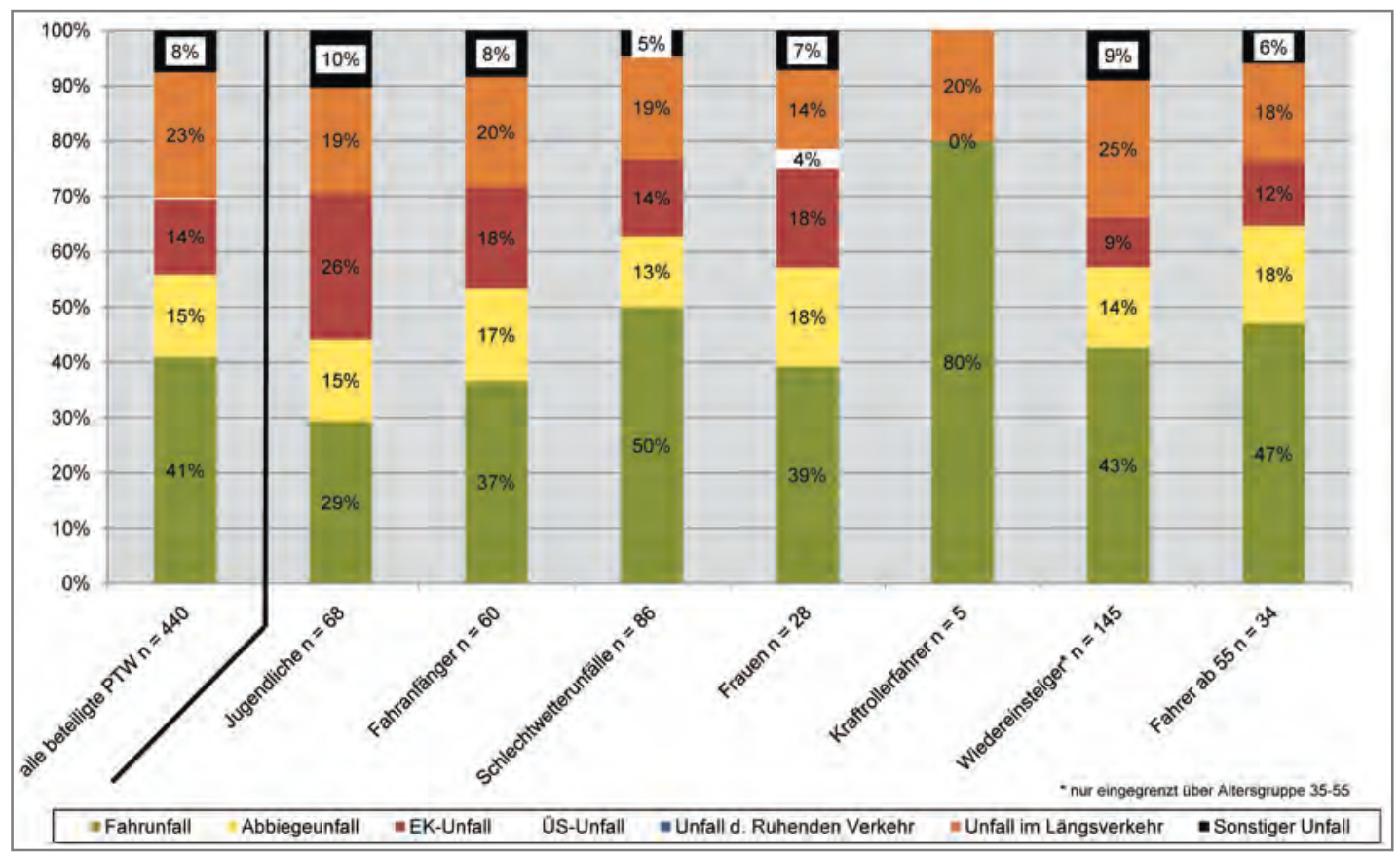

Unfalltypenverteilung der Zielgruppen für alle motorsierten Zweiradfahrer bei Außerortsunfällen auf den festgelegten Streckenabschnitten in Sachsen 
Anlage 06: Überblick über untersuchte Unfallkollektive nach Ortslage, Charakteristik der Unfallstelle und Unfalltyp

Basis: U (P+S) mit Beteiligung motorisierter Zweiräder auf den besichtigten Streckenabschnitten in Sachsen

\begin{tabular}{|c|c|c|c|c|c|c|c|}
\hline Nr. & Bezeichnung & Unf.zah & Bem. & $U(S P) / U(P+S)$ & $U(P) / U(P+S)$ & U (LSA an $) / U(P+S)$ & U (PTW Bet. 01)/U (P+S) \\
\hline 1 & Fahrunfall außerorts in Kurven & 48 & & 0,62 & 0,90 & - & 0.98 \\
\hline 2 & EK-Unfall außerorts an Einmūndungen & 10 & & 0,55 & 0,89 & 0,33 & 0,22 \\
\hline 3 & LV-Unfall außerorts an Steigungsstrecken & 10 & & 0,10 & 0,60 & - & 0,50 \\
\hline 4 & Fahrunfall außerorts an Steigungsstrecken & 9 & $T$ & 0,56 & 0,78 & - & 1,00 \\
\hline 5 & Fahrunfall außerorts an Gefällestrecken & 8 & $T$ & 0,63 & 0,87 & - & 1,00 \\
\hline 6 & LV-Unfall außerorts in Kurven & 10 & & 0,40 & 0,70 & $=$ & 0,60 \\
\hline 7 & Unf. außerorts auf Steigungs- 0 . Gefallestrecken & 38 & & 0,45 & 0,76 & - & 0,76 \\
\hline 8 & LV-Unfall außerorts ohne Charakteristik & 6 & & 0,33 & 0,50 & - & 0,67 \\
\hline 9 & Fahrunfall innerorts in Kurven & 19 & & 0,42 & 0,79 & $=$ & 0,95 \\
\hline 10 & EK-Unfall innerorts an Einmúndungen & 37 & & 0,24 & 0,73 & 0,19 & 0,22 \\
\hline \begin{tabular}{|c|}
11 \\
\end{tabular} & EK-Unfall innerorts an Kreuzungen & 33 & & 0,24 & 0,70 & 0,27 & 0,24 \\
\hline 12 & AB-Unfall innerorts an Einmundungen & 24 & & 0,17 & 0,58 & 0,17 & 0,17 \\
\hline 13 & AB-Unfall innerorts an Kreuzungen & 28 & & 0,25 & 0,71 & 0,57 & 0,21 \\
\hline 14 & LV-Unfall innerorts an Einmündungen & 35 & & 0,09 & 0,46 & 0,26 & 0,71 \\
\hline 15 & LV-Unfall innerorts an Kreuzungen & 37 & & 0,14 & 0,38 & 0,81 & 0,68 \\
\hline 16 & LV-Unfall innerorts an Steigungsstrecken & 10 & & 0,10 & 0.50 & - & 0.70 \\
\hline 17 & LV-Unfall innerorts an Gefallestrecken & 9 & & 0,00 & 0,44 & $=$ & 0,67 \\
\hline 18 & LV-Unfall innerorts in Kurven & 12 & & 0,17 & 0,67 & - & 0,58 \\
\hline 19 & Fahrunfall innerorts ohne Charakteristik & 18 & & 0,33 & 0,89 & - & 0,94 \\
\hline 20 & LV-Unfall innerorts ohne Charakteristik & 54 & & 0,07 & 0,33 & 0,31 & 0,61 \\
\hline
\end{tabular}

gehören vollstăndig ebenso zu Unfallkollektiv (1) 
Anlage 07: Vergleich der Kennwerte zu den Unfallkonstellationen auf Basis unterschiedlicher Kollektivgrößen

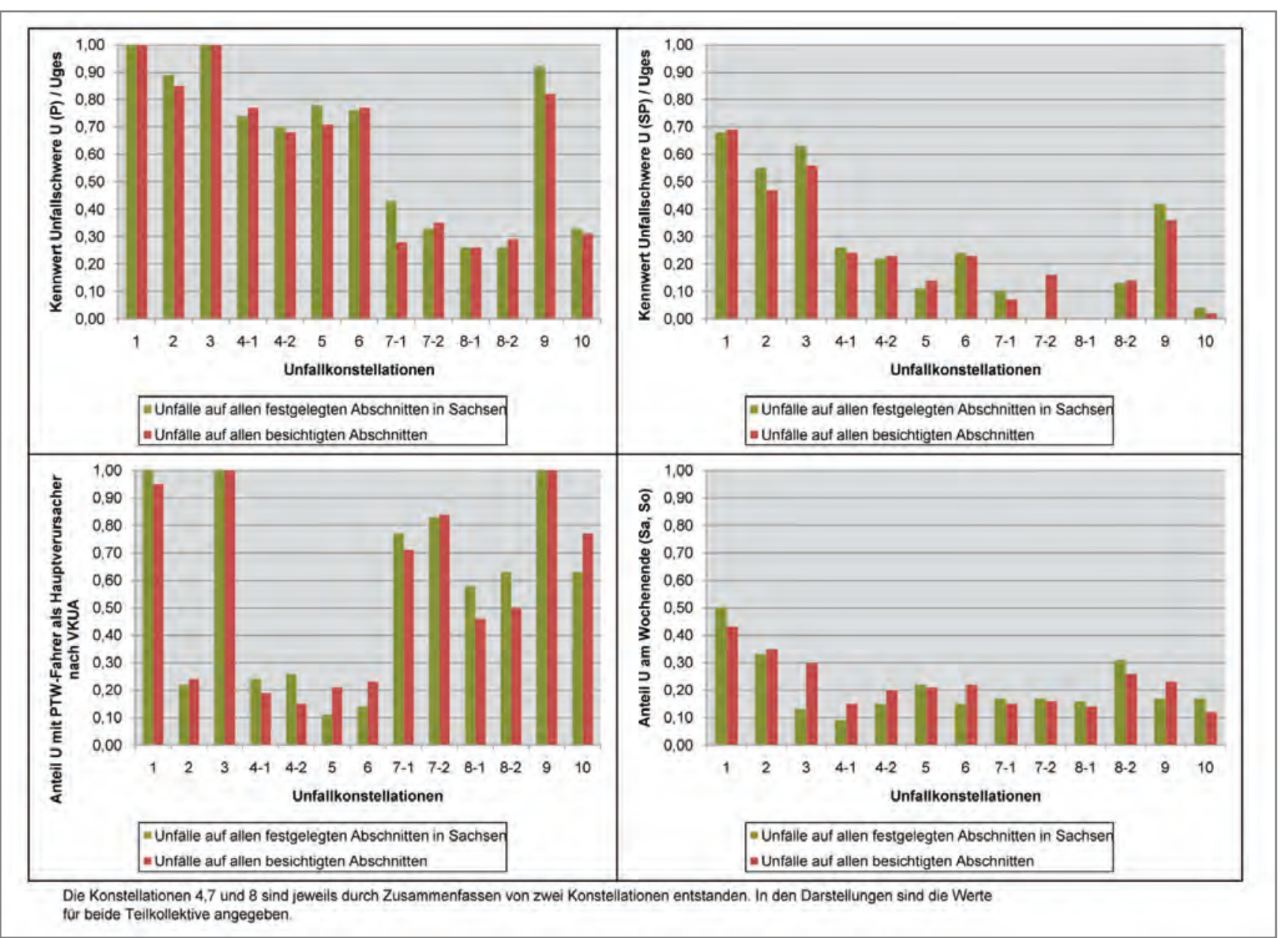




\section{Anlage 08: Detailauswertungen zu den Unfallkonstellationen}

Unfallkonstellation (1): Fahrunfälle außerorts in Kurven mit Abkommen von der Fahrbahn nach rechts sowie gleichzeitig Alleinunfall mit Personenschaden

\begin{tabular}{|l|r|r|}
\hline \multicolumn{3}{|c|}{ Aufteilung nach Unfallart } \\
\hline Art 1 & 0 & $0 \%$ \\
\hline Art 2 & 0 & $0 \%$ \\
\hline Art 3 & 0 & $0 \%$ \\
\hline Art 4 & 0 & $0 \%$ \\
\hline Art 5 & 0 & $0 \%$ \\
\hline Art 6 & 0 & $0 \%$ \\
\hline Art 7 & 0 & $0 \%$ \\
\hline Art 8 & 28 & $100 \%$ \\
\hline Art 9 & 0 & $0 \%$ \\
\hline Art 0 & 0 & $0 \%$ \\
\hline Summe & 28 & $100 \%$ \\
\hline
\end{tabular}

\begin{tabular}{|l|r|r|}
\hline \multicolumn{3}{|c|}{ Aufteilung nach Unfallschwere } \\
\hline U(GT) & 0 & $0 \%$ \\
\hline U (SV) & 19 & $68 \%$ \\
\hline U (LV) & 9 & $32 \%$ \\
\hline U (S) & 0 & $0 \%$ \\
\hline Summe & 28 & $100 \%$ \\
\hline
\end{tabular}

\begin{tabular}{|l|r|r|}
\hline \multicolumn{3}{|c|}{ Aufteilung nach Straßenzustand } \\
\hline nass/feucht & 1 & $4 \%$ \\
\hline winterglatt & 0 & $0 \%$ \\
\hline schlüpfrig & 0 & $0 \%$ \\
\hline trocken & 27 & $96 \%$ \\
\hline Summe & 28 & $100 \%$ \\
\hline
\end{tabular}

\begin{tabular}{|l|r|r|}
\hline \multicolumn{3}{|c|}{ Aufteilung nach Lichtverhältnissen } \\
\hline dunkel/dämmrig & 1 & $4 \%$ \\
\hline hell & 27 & $96 \%$ \\
\hline Summe & 28 & $100 \%$ \\
\hline
\end{tabular}

\begin{tabular}{|l|r|r|}
\hline \multicolumn{3}{|c|}{ Aufteilung nach Aufprall auf Hinderniss } \\
\hline $\mathbf{0}$ (Baum) & 3 & $11 \%$ \\
\hline $\mathbf{1}$ (Mast) & 1 & $4 \%$ \\
\hline $\mathbf{2}$ (Widerlager) & 1 & $\mathbf{4} \%$ \\
\hline 3 (Schutzplanke) & 8 & $29 \%$ \\
\hline $\mathbf{4}$ (sonst. Hindernis) & 6 & $21 \%$ \\
\hline $\mathbf{5}$ (kein Hindernis) & 9 & $32 \%$ \\
\hline Summe & 28 & $100 \%$ \\
\hline
\end{tabular}

\begin{tabular}{|l|r|r|}
\hline \multicolumn{3}{|c|}{ Alleinunfall } \\
\hline PTW-Fahrer & 28 & $100 \%$ \\
\hline Unfälle insgesamt & 28 & $100 \%$ \\
\hline
\end{tabular}

\begin{tabular}{|l|r|r|}
\hline \multicolumn{3}{|c|}{ Hauptverursacher } \\
\hline PTW-Fahrer & 28 & $100 \%$ \\
\hline Unfälle insgesamt & 28 & $100 \%$ \\
\hline
\end{tabular}




\begin{tabular}{|l|r|r|}
\hline \multicolumn{3}{|c|}{ Aufteilung nach Wochentag } \\
\hline $\mathbf{1}$ (Sonntag) & 5 & $18 \%$ \\
\hline $\mathbf{2}$ & 0 & $0 \%$ \\
\hline $\mathbf{3}$ & 3 & $11 \%$ \\
\hline $\mathbf{4}$ & 1 & $4 \%$ \\
\hline $\mathbf{5}$ & 5 & $18 \%$ \\
\hline $\mathbf{6}$ & 5 & $18 \%$ \\
\hline $\mathbf{7}$ & 9 & $32 \%$ \\
\hline Summe & 28 & $100 \%$ \\
\hline
\end{tabular}

\begin{tabular}{|l|r|r|}
\hline \multicolumn{3}{|c|}{ Aufteilung nach Monat } \\
\hline $\mathbf{1}$ (Januar) & 0 & $0 \%$ \\
\hline $\mathbf{2}$ & 1 & $4 \%$ \\
\hline 3 & 0 & $0 \%$ \\
\hline 4 & 2 & $7 \%$ \\
\hline 5 & 2 & $7 \%$ \\
\hline 6 & 3 & $11 \%$ \\
\hline $\mathbf{7}$ & 4 & $14 \%$ \\
\hline $\mathbf{8}$ & 6 & $21 \%$ \\
\hline $\mathbf{9}$ & 5 & $18 \%$ \\
\hline 10 & 4 & $14 \%$ \\
\hline 11 & 1 & $4 \%$ \\
\hline 12 & 0 & $0 \%$ \\
\hline Summe & 28 & $100 \%$ \\
\hline
\end{tabular}

\begin{tabular}{|l|r|r|}
\hline \multicolumn{3}{|c|}{ Aufteilung nach Zielgruppe (bet. PTW-Fahrer) } \\
\hline Jugendliche & 2 & $7 \%$ \\
\hline Fahranfänger & 3 & $11 \%$ \\
\hline Schlechtwetterunf. & 6 & $21 \%$ \\
\hline Frauen & 2 & $7 \%$ \\
\hline Kraftroller & 0 & $0 \%$ \\
\hline Senioren & 2 & $7 \%$ \\
\hline PTW-Fahrer insg. & 28 & $100 \%$ \\
\hline
\end{tabular}

\begin{tabular}{|l|r|r|}
\hline \multicolumn{2}{|c|}{ Unfälle auf Streckenabschnitten mit folgenden Eigenschaften: } \\
\hline mind. 2 Knoten/km & 4 & $14 \%$ \\
\hline mind. 25\% der Abschnittslänge durch Wald oder regelm. Hindernisse & 15 & $54 \%$ \\
\hline Kurvigkeit (KU) > 225 gon/km & 11 & $39 \%$ \\
\hline mind. 1 Hochpunkt (Kuppe) vor einem Knoten oder einer Kurve & 13 & $46 \%$ \\
\hline Steigung/Gefälle nach Z 108/110 StVO & 9 & $32 \%$ \\
\hline Sichteinschränkung vorhanden & 3 & $11 \%$ \\
\hline Querschnitt ohne Mittelmarkierung und unter 6m Breite & 6 & $21 \%$ \\
\hline Kurvigkeit > 225 gon/km und mind. 1 Kuppe vor Knoten/Kurve & 1 & $4 \%$ \\
\hline bestehende Sichteinschränkung und mind. 1 Kuppe vor Knoten/Kurve & 1 & $4 \%$ \\
\hline Kurvigkeit > 225 gon/km und Steigung/Gefälle nach Z 108/110 StVO & 3 & $11 \%$ \\
\hline Querschnitt ohne Mittelmarkierung, < 6m Breite, KU > 225 gon/km & 6 & $21 \%$ \\
\hline Q ohne Mittelm., <6m Breite, KU > 225 gon/km, Steigung/Gef. n. VZ & 1 & $4 \%$ \\
\hline Unfälle insgesamt & 28 & $100 \%$ \\
\hline
\end{tabular}


Unfallkonstellation (2): Einbiegen/Kreuzen-Unfälle außerorts an Kurven/Einmündungen

\begin{tabular}{|l|r|r|}
\hline \multicolumn{3}{|c|}{ Aufteilung nach Unfallart } \\
\hline Art 1 & 0 & $0 \%$ \\
\hline Art 2 & 0 & $0 \%$ \\
\hline Art 3 & 0 & $0 \%$ \\
\hline Art 4 & 0 & $0 \%$ \\
\hline Art 5 & 9 & $100 \%$ \\
\hline Art 6 & 0 & $0 \%$ \\
\hline Art 7 & 0 & $0 \%$ \\
\hline Art 8 & 0 & $0 \%$ \\
\hline Art 9 & 0 & $0 \%$ \\
\hline Art 0 & 0 & $0 \%$ \\
\hline Summe & 9 & $100 \%$ \\
\hline
\end{tabular}

\begin{tabular}{|l|r|r|}
\hline \multicolumn{3}{|c|}{ Aufteilung nach Unfallschwere } \\
\hline $\mathbf{U}(\mathrm{GT})$ & 1 & $11 \%$ \\
\hline $\mathbf{U}(\mathrm{SV})$ & 4 & $44 \%$ \\
\hline $\mathbf{U}(\mathrm{LV})$ & 3 & $33 \%$ \\
\hline $\mathbf{U}(\mathbf{S})$ & 1 & $11 \%$ \\
\hline Summe & 9 & $100 \%$ \\
\hline
\end{tabular}

\begin{tabular}{|l|r|r|}
\hline \multicolumn{3}{|c|}{ Aufteilung nach Straßenzustand } \\
\hline nass/feucht & 1 & $11 \%$ \\
\hline winterglatt & 0 & $0 \%$ \\
\hline schlüpfrig & 0 & $0 \%$ \\
\hline trocken & 8 & $89 \%$ \\
\hline Summe & 9 & $100 \%$ \\
\hline
\end{tabular}

\begin{tabular}{|l|r|r|}
\hline \multicolumn{3}{|c|}{ Aufteilung nach Lichtverhältnissen } \\
\hline dunkel/dämmrig & 2 & $22 \%$ \\
\hline hell & 7 & $78 \%$ \\
\hline Summe & 9 & $100 \%$ \\
\hline
\end{tabular}

\begin{tabular}{|l|r|r|}
\hline \multicolumn{3}{|c|}{ Aufteilung nach LSA-Merkmal } \\
\hline LSA an & 3 & $33 \%$ \\
\hline LSA aus & 1 & $11 \%$ \\
\hline Unfälle insgesamt & 9 & $100 \%$ \\
\hline
\end{tabular}
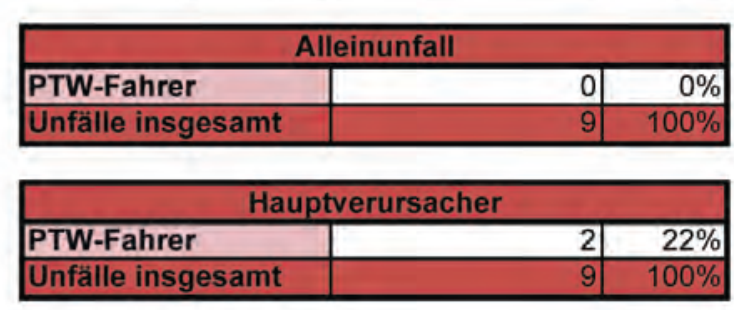

\begin{tabular}{|l|r|r|}
\hline \multicolumn{3}{|c|}{ Aufteilung nach Wochentag } \\
\hline $\mathbf{1}$ (Sonntag) & 1 & $11 \%$ \\
\hline $\mathbf{2}$ & 1 & $11 \%$ \\
\hline $\mathbf{3}$ & 1 & $11 \%$ \\
\hline $\mathbf{4}$ & 1 & $11 \%$ \\
\hline $\mathbf{5}$ & 2 & $22 \%$ \\
\hline $\mathbf{6}$ & 1 & $11 \%$ \\
\hline $\mathbf{7}$ & 2 & $22 \%$ \\
\hline
\end{tabular}




\begin{tabular}{|l|r|r|}
\hline \multicolumn{3}{|c|}{ Aufteilung nach Monat } \\
\hline $\mathbf{1}$ (Januar) & 0 & $0 \%$ \\
\hline $\mathbf{2}$ & 0 & $0 \%$ \\
\hline 3 & 1 & $11 \%$ \\
\hline $\mathbf{4}$ & 0 & $0 \%$ \\
\hline $\mathbf{5}$ & 2 & $22 \%$ \\
\hline 6 & 0 & $0 \%$ \\
\hline $\mathbf{7}$ & 0 & $0 \%$ \\
\hline $\mathbf{8}$ & 0 & $0 \%$ \\
\hline $\mathbf{9}$ & 3 & $33 \%$ \\
\hline 10 & 3 & $33 \%$ \\
\hline 11 & 0 & $0 \%$ \\
\hline 12 & 0 & $0 \%$ \\
\hline Summe & 9 & $100 \%$ \\
\hline
\end{tabular}

\begin{tabular}{|l|r|r|}
\hline \multicolumn{3}{|c|}{ Aufteilung nach Zielgruppe (bet. PTW-Fahrer) } \\
\hline Jugendliche & 1 & $11 \%$ \\
\hline Fahranfänger & 1 & $11 \%$ \\
\hline Schlechtwetterunf. & 2 & $22 \%$ \\
\hline Frauen & 1 & $11 \%$ \\
\hline Kraftroller & & $0 \%$ \\
\hline Senioren & 1 & $11 \%$ \\
\hline PTW-Fahrer insg. & 9 & $100 \%$ \\
\hline
\end{tabular}

\begin{tabular}{|l|r|r|}
\hline \multicolumn{2}{|c|}{ Unfälle auf Streckenabschnitten mit folgenden Eigenschaften: } \\
\hline mind. 2 Knoten/km & 3 & $33 \%$ \\
\hline mind. 25\% der Abschnittslänge durch Wald oder regelm. Hindernisse & 2 & $22 \%$ \\
\hline Kurvigkeit (KU) > 225 gon/km & 1 & $11 \%$ \\
\hline mind. 1 Hochpunkt (Kuppe) vor einem Knoten oder einer Kurve & 3 & $33 \%$ \\
\hline Steigung/Gefälle nach Z 108/110 StVO & 1 & $11 \%$ \\
\hline Sichteinschränkung vorhanden & 2 & $22 \%$ \\
\hline Querschnitt ohne Mittelmarkierung und unter 6m Breite & 0 & $0 \%$ \\
\hline Kurvigkeit > 225 gon/km und mind. 1 Kuppe vor Knoten/Kurve & & nicht untersucht \\
\hline bestehende Sichteinschränkung und mind. 1 Kuppe vor Knoten/Kurve & & nicht untersucht \\
\hline Kurvigkeit > 225 gon/km und Steigung/Gefälle nach Z 108/110 StVO & & nicht untersucht \\
\hline Querschnitt ohne Mittelmarkierung, < 6m Breite, KU > 225 gon/km & & nicht untersucht \\
\hline Q ohne Mittelm., < 6m Breite, KU > 225 gon/km, Steigung/Gef. $\mathbf{n}$. VZ & & nicht untersucht \\
\hline Unfälle insgesamt & 9 & $100 \%$ \\
\hline
\end{tabular}


Unfallkonstellation (3): Fahrunfälle innerorts an Kurven mit Abkommen von der Fahrbahn nach rechts sowie gleichzeitig Alleinunfall mit Personennschaden

\begin{tabular}{|l|r|r|}
\hline \multicolumn{3}{|c|}{ Aufteilung nach Unfallart } \\
\hline Art 1 & 0 & $0 \%$ \\
\hline Art 2 & 0 & $0 \%$ \\
\hline Art 3 & 0 & $0 \%$ \\
\hline Art 4 & 0 & $0 \%$ \\
\hline Art 5 & 0 & $0 \%$ \\
\hline Art 6 & 0 & $0 \%$ \\
\hline Art 7 & 0 & $0 \%$ \\
\hline Art 8 & 8 & $100 \%$ \\
\hline Art 9 & 0 & $0 \%$ \\
\hline Art 0 & 0 & $0 \%$ \\
\hline Summo & 8 & $10 n \%$ \\
\hline
\end{tabular}

\begin{tabular}{|l|r|r|}
\hline \multicolumn{3}{|c|}{ Aufteilung nach Unfallschwere } \\
\hline U(GT) & 1 & $13 \%$ \\
\hline U (SV) & 4 & $50 \%$ \\
\hline U (LV) & 3 & $38 \%$ \\
\hline U (S) & 0 & $0 \%$ \\
\hline Summe & 8 & $100 \%$ \\
\hline
\end{tabular}

\begin{tabular}{|l|r|r|}
\hline \multicolumn{3}{|c|}{ Aufteilung nach Straßenzustand } \\
\hline nass/feucht & 0 & $0 \%$ \\
\hline winterglatt & 0 & $0 \%$ \\
\hline schlüpfrig & 0 & $0 \%$ \\
\hline trocken & 8 & $100 \%$ \\
\hline Summe & 8 & $100 \%$ \\
\hline
\end{tabular}

\begin{tabular}{|l|r|r|}
\hline \multicolumn{3}{|c|}{ Aufteilung nach Lichtverhältnissen } \\
\hline dunkel/dämmrig & 3 & $38 \%$ \\
\hline hell & 5 & $63 \%$ \\
\hline Summe & 8 & $100 \%$ \\
\hline
\end{tabular}

\begin{tabular}{|l|r|r|}
\hline \multicolumn{3}{|c|}{ Aufteilung nach Aufprall auf Hinderniss } \\
\hline $\mathbf{0}$ (Baum) & 0 & $\mathbf{0}$ \\
\hline $\mathbf{1}$ (Mast) & 0 & $\mathbf{0}$ \\
\hline $\mathbf{2}$ (Widerlager) & 0 & $\mathbf{0} \%$ \\
\hline $\mathbf{3}$ (Schutzplanke) & 0 & $0 \%$ \\
\hline $\mathbf{4}$ (sonst. Hindernis) & 0 & $0 \%$ \\
\hline $\mathbf{5}$ (kein Hindernis) & 8 & $100 \%$ \\
\hline Summe & 8 & $100 \%$ \\
\hline
\end{tabular}

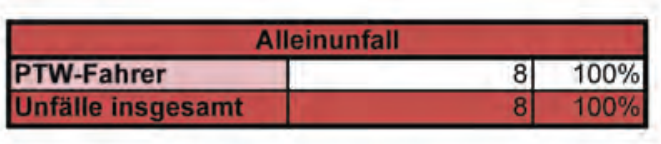

\begin{tabular}{|l|r|r|}
\hline \multicolumn{3}{|c|}{ Hauptverursacher } \\
\hline PTW-Fahrer & 8 & $100 \%$ \\
\hline Unfälle insgesamt & 8 & $100 \%$ \\
\hline
\end{tabular}

\begin{tabular}{|l|r|r|}
\hline \multicolumn{3}{|c|}{ Aufteilung nach Wochentag } \\
\hline $\mathbf{1}$ (Sonntag) & 1 & $13 \%$ \\
\hline $\mathbf{2}$ & 0 & $0 \%$ \\
\hline $\mathbf{3}$ & 0 & $0 \%$ \\
\hline $\mathbf{4}$ & 0 & $0 \%$ \\
\hline $\mathbf{5}$ & 3 & $38 \%$ \\
\hline $\mathbf{6}$ & 4 & $50 \%$ \\
\hline $\mathbf{7}$ & 0 & $0 \%$ \\
\hline Summe & 8 & $100 \%$ \\
\hline
\end{tabular}




\begin{tabular}{|l|r|r|}
\hline \multicolumn{3}{|c|}{ Aufteilung nach Monat } \\
\hline $\mathbf{1}$ (Januar) & 0 & $0 \%$ \\
\hline $\mathbf{2}$ & 0 & $0 \%$ \\
\hline $\mathbf{3}$ & 0 & $0 \%$ \\
\hline $\mathbf{4}$ & 0 & $0 \%$ \\
\hline $\mathbf{5}$ & 2 & $25 \%$ \\
\hline $\mathbf{6}$ & 1 & $13 \%$ \\
\hline $\mathbf{7}$ & 1 & $13 \%$ \\
\hline $\mathbf{8}$ & 1 & $13 \%$ \\
\hline $\mathbf{9}$ & 2 & $25 \%$ \\
\hline $\mathbf{1 0}$ & 0 & $0 \%$ \\
\hline $\mathbf{1 1}$ & 1 & $13 \%$ \\
\hline $\mathbf{1 2}$ & 0 & $0 \%$ \\
\hline Summe & 8 & $100 \%$ \\
\hline
\end{tabular}

\begin{tabular}{|l|r|r|}
\hline \multicolumn{3}{|c|}{ Aufteilung nach Zielgruppe (bet. PTW-Fahrer) } \\
\hline Jugendliche & 1 & $13 \%$ \\
\hline Fahranfänger & 1 & $13 \%$ \\
\hline Schlechtwetterunf. & 1 & $13 \%$ \\
\hline Frauen & 2 & $25 \%$ \\
\hline Kraftroller & 0 & $0 \%$ \\
\hline Senioren & 2 & $25 \%$ \\
\hline PTW-Fahrer insg. & 8 & $100 \%$ \\
\hline
\end{tabular}

\begin{tabular}{|l|r|r|}
\hline \multicolumn{2}{|c|}{ Unfälle auf Streckenabschnitten mit folgenden Eigenschaften: } & $38 \%$ \\
\hline mindestens 4 Knoten/km & 3 & $25 \%$ \\
\hline erhebliche Mängel im Straßenzustand & 2 & $13 \%$ \\
\hline Straße mit Straßenbahn auf der Fahrbahn & 1 & $0 \%$ \\
\hline Querschnitt mit baulich abgesetzter Mitteltrennung & 0 & $13 \%$ \\
\hline mindestens 2 Fahrstreifen pro Richtung & 1 & $38 \%$ \\
\hline Uückenhafte oder keine angrenzende Bebauung & 3 & $13 \%$ \\
\hline kreuzende Gleisanlagen & 1 & $0 \%$ \\
\hline Parkflächen am Fahrbahnrand & 0 & $0 \%$ \\
\hline Zulässige Höchstgeschwindigkeit über 50 km/h & 0 & $0 \%$ \\
\hline Zulässige Höchstgeschwindigkeit 30 km/h oder kleiner & 0 & $75 \%$ \\
\hline Fahrbahnrand: Bordstein & 6 & $100 \%$ \\
\hline Unfälle insgesamt & 8 & \\
\hline
\end{tabular}


Unfallkonstellation (4): Einbiegen/Kreuzen-Unfälle innerorts an Kreuzungen/Einmündungen

\begin{tabular}{|l|r|r|}
\hline \multicolumn{3}{|c|}{ Aufteilung nach Unfallart } \\
\hline Art 1 & 0 & $0 \%$ \\
\hline Art 2 & 0 & $0 \%$ \\
\hline Art 3 & 0 & $0 \%$ \\
\hline Art 4 & 0 & $0 \%$ \\
\hline Art 5 & 61 & $100 \%$ \\
\hline Art 6 & 0 & $0 \%$ \\
\hline Art 7 & 0 & $0 \%$ \\
\hline Art 8 & 0 & $0 \%$ \\
\hline Art 9 & 0 & $0 \%$ \\
\hline Art 0 & 0 & $0 \%$ \\
\hline Summe & 61 & $218 \%$ \\
\hline
\end{tabular}

\begin{tabular}{|l|r|r|}
\hline \multicolumn{3}{|c|}{ Aufteilung nach Unfallschwere } \\
\hline $\mathbf{U}(\mathrm{GT})$ & 0 & $0 \%$ \\
\hline $\mathbf{U}(\mathrm{SV})$ & 15 & $25 \%$ \\
\hline $\mathbf{U}(\mathrm{LV})$ & 29 & $48 \%$ \\
\hline $\mathbf{U}(\mathbf{S})$ & 17 & $28 \%$ \\
\hline Summe & 61 & $100 \%$ \\
\hline
\end{tabular}

\begin{tabular}{|l|r|r|}
\hline \multicolumn{3}{|c|}{ Aufteilung nach Straßenzustand } \\
\hline nass/feucht & 8 & $13 \%$ \\
\hline winterglatt & 0 & $0 \%$ \\
\hline schlüpfrig & 0 & $0 \%$ \\
\hline trocken & 53 & $87 \%$ \\
\hline Summe & 61 & $100 \%$ \\
\hline
\end{tabular}
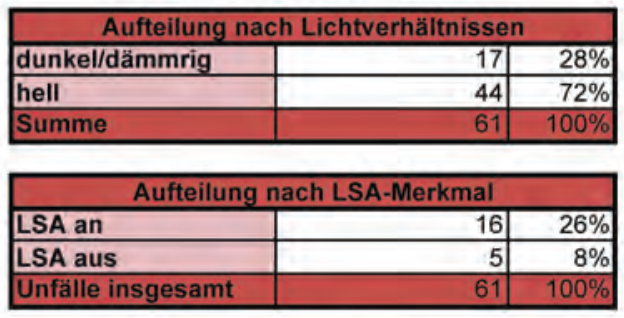

\begin{tabular}{|l|r|l|}
\hline \multicolumn{3}{|c|}{ Aufteilung nach Aufprall auf Hinderniss } \\
\hline 0 (Baum) & nicht untersucht & \\
\hline 1 (Mast) & nicht untersucht & \\
\hline 2 (Widerlager) & nicht untersucht & \\
\hline 3 (Schutzplanke) & nicht untersucht & \\
\hline 4 (sonst. Hindernis) & nicht untersucht & \\
\hline 5 (kein Hindernis) & nicht untersucht & \\
\hline Summe & & \\
\hline
\end{tabular}

\begin{tabular}{|l|r|r|}
\hline \multicolumn{2}{|c|}{ Alleinunfall } & 0 \\
\hline PTW-Fahrer & 61 & $0 \%$ \\
\hline Unfälle insgesamt & & $100 \%$ \\
\hline
\end{tabular}

\begin{tabular}{|l|r|r|}
\hline \multicolumn{3}{|l|}{ Hauptverursacher } \\
\hline PTW-Fahrer & 15 & $25 \%$ \\
\hline Unfälle insgesamt & 61 & $100 \%$ \\
\hline
\end{tabular}

\begin{tabular}{|l|r|r|}
\hline \multicolumn{3}{|c|}{ Aufteilung nach Wochentag } \\
\hline $\mathbf{1}$ (Sonntag) & 5 & $8 \%$ \\
\hline $\mathbf{2}$ & 7 & $11 \%$ \\
\hline 3 & 11 & $18 \%$ \\
\hline 4 & 13 & $21 \%$ \\
\hline 5 & 7 & $11 \%$ \\
\hline 6 & 16 & $26 \%$ \\
\hline 7 & 2 & $3 \%$ \\
\hline Summe & 61 & $100 \%$ \\
\hline
\end{tabular}




\begin{tabular}{|c|c|c|}
\hline \multicolumn{3}{|c|}{ Aufteilung nach Monat } \\
\hline 1 (Januar) & 0 & $0 \%$ \\
\hline 2 & 1 & $2 \%$ \\
\hline 3 & 2 & $3 \%$ \\
\hline 4 & 10 & $16 \%$ \\
\hline 5 & 9 & $15 \%$ \\
\hline 6 & 10 & $16 \%$ \\
\hline 7 & 10 & $16 \%$ \\
\hline 8 & 2 & $3 \%$ \\
\hline 9 & 9 & $15 \%$ \\
\hline 10 & 4 & $7 \%$ \\
\hline 11 & 2 & $3 \%$ \\
\hline 12 & 2 & $3 \%$ \\
\hline Summe & 61 & $100 \%$ \\
\hline
\end{tabular}

\begin{tabular}{|l|r|r|}
\hline \multicolumn{3}{|c|}{ Aufteilung nach Zielgruppe (bet. PTW-Fahrer) } \\
\hline Jugendliche & 14 & $22 \%$ \\
\hline Fahranfänger & 13 & $20 \%$ \\
\hline Schlechtwetterunf. & 13 & $20 \%$ \\
\hline Frauen & 5 & $8 \%$ \\
\hline Kraftroller & 0 & $0 \%$ \\
\hline Senioren & 3 & $5 \%$ \\
\hline PTW-Fahrer insg. & 64 & $100 \%$ \\
\hline
\end{tabular}

\begin{tabular}{|l|r|r|}
\hline \multicolumn{2}{|c|}{ Unfälle auf Streckenabschnitten mit folgenden Eigenschaften: } \\
\hline mindestens 4 Knoten/km & 38 & 16 \\
\hline erhebliche Mängel im Straßenzustand & 27 & $62 \%$ \\
\hline Straße mit Straßenbahn auf der Fahrbahn & 4 & $26 \%$ \\
\hline Querschnitt mit baulich abgesetzter Mitteltrennung & 7 & $44 \%$ \\
\hline mindestens 2 Fahrstreifen pro Richtung & 42 & $7 \%$ \\
\hline lückenhafte oder keine angrenzende Bebauung & 22 & $11 \%$ \\
\hline kreuzende Gleisanlagen & 14 & \\
\hline Parkflächen am Fahrbahnrand & 2 & \\
\hline Zulässige Höchstgeschwindigkeit über 50 km/h & 1 & $36 \%$ \\
\hline Zulässige Höchstgeschwindigkeit 30 km/h oder kleiner & 51 & $23 \%$ \\
\hline Fahrbahnrand: Bordstein & 61 & $3 \%$ \\
\hline Unfälle insgesamt & $2 \%$ \\
\hline
\end{tabular}


Unfallkonstellation (5): Abbiegeunfall innerorts an Einmündungen mit Unfallart 3

\begin{tabular}{|l|r|r|}
\hline \multicolumn{3}{|c|}{ Aufteilung nach Unfallart } \\
\hline Art 1 & & $0 \%$ \\
\hline Art 2 & & $0 \%$ \\
\hline Art 3 & & $100 \%$ \\
\hline Art 4 & & $0 \%$ \\
\hline Art 5 & & $0 \%$ \\
\hline Art 6 & & $0 \%$ \\
\hline Art 7 & & $0 \%$ \\
\hline Art 8 & & $0 \%$ \\
\hline Art 9 & & $0 \%$ \\
\hline Art 0 & & $0 \%$ \\
\hline Summe & & $100 \%$ \\
\hline
\end{tabular}

\begin{tabular}{|l|r|r|}
\hline \multicolumn{3}{|c|}{ Aufteilung nach Unfallschwere } \\
\hline $\mathbf{U}(\mathrm{GT})$ & 0 & $0 \%$ \\
\hline $\mathbf{U}(\mathrm{SV})$ & 1 & $11 \%$ \\
\hline $\mathbf{U}(\mathrm{LV})$ & 6 & $67 \%$ \\
\hline $\mathbf{U}(\mathrm{S})$ & 2 & $22 \%$ \\
\hline Summe & 9 & $100 \%$ \\
\hline
\end{tabular}

\begin{tabular}{|l|r|r|}
\hline \multicolumn{3}{|c|}{ Aufteilung nach Straßenzustand } \\
\hline nass/feucht & 0 & $0 \%$ \\
\hline winterglatt & 0 & $0 \%$ \\
\hline schlüpfrig & 0 & $0 \%$ \\
\hline trocken & 9 & $100 \%$ \\
\hline Summe & 9 & $100 \%$ \\
\hline
\end{tabular}

\begin{tabular}{|l|r|r|}
\hline \multicolumn{3}{|c|}{ Aufteilung nach Lichtverhältnissen } \\
\hline dunkel/dämmrig & 1 & $11 \%$ \\
\hline hell & 8 & $89 \%$ \\
\hline Summe & 9 & $100 \%$ \\
\hline
\end{tabular}

\begin{tabular}{|l|r|r|}
\hline \multicolumn{3}{|c|}{ Aufteilung nach LSA-Merkmal } \\
\hline LSA an & 1 & $11 \%$ \\
\hline LSA aus & 0 & $0 \%$ \\
\hline Unfälle insgesamt & 9 & $100 \%$ \\
\hline
\end{tabular}
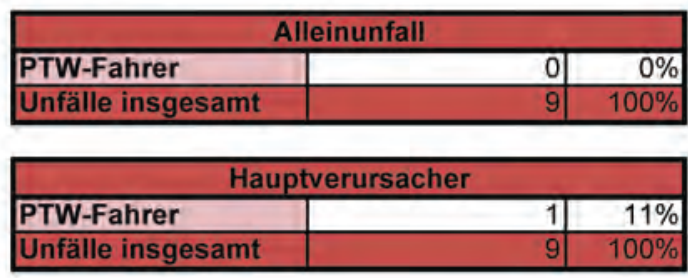

\begin{tabular}{|l|r|r|}
\hline \multicolumn{3}{|c|}{ Aufteilung nach Wochentag } \\
\hline $\mathbf{1}$ (Sonntag) & 1 & $11 \%$ \\
\hline $\mathbf{2}$ & 0 & $0 \%$ \\
\hline $\mathbf{3}$ & 2 & $22 \%$ \\
\hline $\mathbf{4}$ & 1 & $11 \%$ \\
\hline $\mathbf{5}$ & 1 & $11 \%$ \\
\hline $\mathbf{6}$ & 3 & $33 \%$ \\
\hline $\mathbf{7}$ & 1 & $11 \%$ \\
\hline Summe & 9 & $100 \%$ \\
\hline
\end{tabular}




\begin{tabular}{|l|r|r|}
\hline \multicolumn{3}{|c|}{ Aufteilung nach Monat } \\
\hline $\mathbf{1}$ (Januar) & 0 & $0 \%$ \\
\hline $\mathbf{3}$ & 0 & $0 \%$ \\
\hline 3 & 0 & $0 \%$ \\
\hline 4 & 0 & $0 \%$ \\
\hline 5 & 2 & $22 \%$ \\
\hline 6 & 0 & $0 \%$ \\
\hline $\mathbf{7}$ & 2 & $22 \%$ \\
\hline 8 & 1 & $11 \%$ \\
\hline 9 & 2 & $22 \%$ \\
\hline 10 & 1 & $11 \%$ \\
\hline 11 & 0 & $0 \%$ \\
\hline 12 & 1 & $11 \%$ \\
\hline & & \\
\hline
\end{tabular}

\begin{tabular}{|l|r|r|}
\hline \multicolumn{2}{|c|}{ Aufteilung nach Zielgruppe (bet. PTW-Fahrer) } \\
\hline Jugendliche & 1 & $10 \%$ \\
\hline Fahranfänger & 1 & $10 \%$ \\
\hline Schlechtwetterunf. & 1 & $10 \%$ \\
\hline Frauen & 2 & $20 \%$ \\
\hline Kraftroller & 0 & $0 \%$ \\
\hline Senioren & 0 & $0 \%$ \\
\hline PTW-Fahrer insg. & 10 & $100 \%$ \\
\hline
\end{tabular}

\begin{tabular}{|l|r|r|}
\hline \multicolumn{2}{|c|}{ Unfälle auf Streckenabschnitten mit folgenden Eigenschaften: } \\
\hline mindestens 4 Knoten/km & 7 & 5 \\
\hline erhebliche Mängel im Straßenzustand & 6 & $78 \%$ \\
\hline Straße mit Straßenbahn auf der Fahrbahn & 1 & $56 \%$ \\
\hline Querschnitt mit baulich abgesetzter Mitteltrennung & 1 & $67 \%$ \\
\hline mindestens 2 Fahrstreifen pro Richtung & 7 & $11 \%$ \\
\hline lückenhafte oder keine angrenzende Bebauung & 1 & $11 \%$ \\
\hline kreuzende Gleisanlagen & 2 & $78 \%$ \\
\hline Parkflächen am Fahrbahnrand & 0 & $11 \%$ \\
\hline zulässige Höchstgeschwindigkeit über 50 km/h & 0 & $22 \%$ \\
\hline zulässige Höchstgeschwindigkeit 30 km/h oder kleiner & 8 & $0 \%$ \\
\hline Fahrbahnrand: Bordstein & 9 & $0 \%$ \\
\hline Unfälle insgesamt & $89 \%$ \\
\hline
\end{tabular}


Unfallkonstellation (6): Abbiegeunfall innerorts an Kreuzungen mit Unfallart 4 oder 5

\begin{tabular}{|l|r|r|}
\hline \multicolumn{2}{|c|}{ Aufteilung nach Unfallart } \\
\hline Art 1 & $0 \%$ \\
\hline Art 2 & & $0 \%$ \\
\hline Art 3 & 7 & $0 \%$ \\
\hline Art 4 & $33 \%$ \\
\hline Art 5 & 14 & $67 \%$ \\
\hline Art 6 & & $0 \%$ \\
\hline Art 7 & & $0 \%$ \\
\hline Art 8 & & $0 \%$ \\
\hline Art 9 & & $0 \%$ \\
\hline Art 0 & & $0 \%$ \\
\hline Summe & & $100 \%$ \\
\hline
\end{tabular}

\begin{tabular}{|l|r|r|}
\hline \multicolumn{3}{|c|}{ Aufteilung nach Unfallschwere } \\
\hline $\mathbf{U}(\mathrm{GT})$ & 0 & $0 \%$ \\
\hline $\mathbf{U}(\mathrm{SV})$ & 5 & $24 \%$ \\
\hline $\mathbf{U}(\mathbf{L V})$ & 11 & $52 \%$ \\
\hline $\mathbf{U}(\mathbf{S})$ & 5 & $24 \%$ \\
\hline Summe & 21 & $100 \%$ \\
\hline
\end{tabular}

\begin{tabular}{|l|r|r|}
\hline \multicolumn{3}{|c|}{ Aufteilung nach Straßenzustand } \\
\hline nass/feucht & 5 & $24 \%$ \\
\hline winterglatt & 0 & $0 \%$ \\
\hline schlüpfrig & 0 & $0 \%$ \\
\hline trocken & 16 & $76 \%$ \\
\hline Summe & 21 & $100 \%$ \\
\hline
\end{tabular}

\begin{tabular}{|l|r|r|}
\hline \multicolumn{3}{|c|}{ Aufteilung nach Lichtverhältnissen } \\
\hline dunkel/dämmrig & 5 & $24 \%$ \\
\hline hell & 16 & $76 \%$ \\
\hline Summe & 21 & $100 \%$ \\
\hline
\end{tabular}

\begin{tabular}{|l|r|r|}
\hline \multicolumn{3}{|c|}{ Aufteilung nach LSA-Merkmal } \\
\hline LSA an & 13 & $62 \%$ \\
\hline LSA aus & 2 & $10 \%$ \\
\hline Unfälle insgesamt & 21 & $100 \%$ \\
\hline
\end{tabular}

\begin{tabular}{|l|r|r|}
\hline \multicolumn{3}{|c|}{ Alleinunfall } \\
\hline PTW-Fahrer & 0 & $0 \%$ \\
\hline Unfälle insgesamt & 21 & $100 \%$ \\
\hline
\end{tabular}

\begin{tabular}{|l|r|r|}
\hline \multicolumn{3}{|c|}{ Hauptverursacher } \\
\hline PTW-Fahrer & 3 & $14 \%$ \\
\hline Unfälle insgesamt & 21 & $100 \%$ \\
\hline
\end{tabular}

\begin{tabular}{|l|r|r|}
\hline \multicolumn{3}{|c|}{ Aufteilung nach Wochentag } \\
\hline $\mathbf{1}$ (Sonntag) & 2 & $10 \%$ \\
\hline $\mathbf{2}$ & 2 & $10 \%$ \\
\hline $\mathbf{3}$ & 3 & $14 \%$ \\
\hline $\mathbf{4}$ & 2 & $10 \%$ \\
\hline $\mathbf{5}$ & 5 & $24 \%$ \\
\hline 6 & 6 & $29 \%$ \\
\hline $\mathbf{7}$ & 1 & $5 \%$ \\
\hline Summe & 21 & $100 \%$ \\
\hline
\end{tabular}




\begin{tabular}{|l|r|r|}
\hline \multicolumn{3}{|c|}{ Aufteilung nach Monat } \\
\hline $\mathbf{1}$ (Januar) & 0 & $0 \%$ \\
\hline $\mathbf{2}$ & 0 & $0 \%$ \\
\hline $\mathbf{3}$ & 0 & $0 \%$ \\
\hline $\mathbf{4}$ & 1 & $5 \%$ \\
\hline $\mathbf{5}$ & 2 & $10 \%$ \\
\hline $\mathbf{6}$ & 1 & $5 \%$ \\
\hline $\mathbf{7}$ & 3 & $14 \%$ \\
\hline $\mathbf{9}$ & 6 & $29 \%$ \\
\hline $\mathbf{1 0}$ & 2 & $10 \%$ \\
\hline 11 & 3 & $14 \%$ \\
\hline 12 & 3 & $14 \%$ \\
\hline Summe & 0 & $0 \%$ \\
\hline
\end{tabular}

\begin{tabular}{|l|r|r|}
\hline \multicolumn{3}{|c|}{ Aufteilung nach Zielgruppe (bet. PTW-Fahrer) } \\
\hline Jugendliche & 5 & $23 \%$ \\
\hline Fahranfänger & 3 & $14 \%$ \\
\hline Schlechtwetterunf. & 8 & $36 \%$ \\
\hline Frauen & 1 & $5 \%$ \\
\hline Kraftroller & 0 & $0 \%$ \\
\hline Senioren & 2 & $9 \%$ \\
\hline PTW-Fahrer insg. & 22 & $100 \%$ \\
\hline
\end{tabular}

\begin{tabular}{|l|r|r|}
\hline \multicolumn{2}{|c|}{ Unfälle auf Streckenabschnitten mit folgenden Eigenschaften: } \\
\hline mindestens 4 Knoten/km & 15 & $71 \%$ \\
\hline erhebliche Mängel im Straßenzustand & 8 & $38 \%$ \\
\hline Straße mit Straßenbahn auf der Fahrbahn & 11 & $52 \%$ \\
\hline Querschnitt mit baulich abgesetzter Mitteltrennung & 0 & $0 \%$ \\
\hline mindestens 2 Fahrstreifen pro Richtung & 4 & $19 \%$ \\
\hline lückenhafte oder keine angrenzende Bebauung & 11 & $52 \%$ \\
\hline kreuzende Gleisanlagen & 4 & 1 \\
\hline Parkflächen am Fahrbahnrand & 1 & \\
\hline zulässige Höchstgeschwindigkeit über 50 km/h & 5 & $5 \%$ \\
\hline zulässige Höchstgeschwindigkeit 30 km/h oder kleiner & 0 & $24 \%$ \\
\hline Fahrbahnrand: Bordstein & 21 & $0 \%$ \\
\hline Unfälle insgesamt & $90 \%$ \\
\hline
\end{tabular}


Unfallkonstellation (7): Auffahrunfall innerorts an Kreuzungen, Einmündungen oder Gefällestrecken

\begin{tabular}{|l|r|r|}
\hline \multicolumn{3}{|c|}{ Aufteilung nach Unfallart } \\
\hline Art 1 & 0 & $0 \%$ \\
\hline Art 2 & 36 & $100 \%$ \\
\hline Art 3 & 0 & $0 \%$ \\
\hline Art 4 & 0 & $0 \%$ \\
\hline Art 5 & 0 & $0 \%$ \\
\hline Art 6 & 0 & $0 \%$ \\
\hline Art 7 & 0 & $0 \%$ \\
\hline Art 8 & 0 & $0 \%$ \\
\hline Art 9 & 0 & $0 \%$ \\
\hline Art 0 & 0 & $0 \%$ \\
\hline Summe & 36 & $100 \%$ \\
\hline
\end{tabular}

\begin{tabular}{|l|r|r|}
\hline \multicolumn{3}{|c|}{ Aufteilung nach Unfallschwere } \\
\hline $\mathbf{U}(\mathbf{G T})$ & 0 & $0 \%$ \\
\hline $\mathbf{U}(\mathrm{SV})$ & 3 & $8 \%$ \\
\hline $\mathbf{U}(\mathrm{LV})$ & 12 & $33 \%$ \\
\hline $\mathbf{U}(\mathbf{S})$ & 21 & $58 \%$ \\
\hline Summe & 36 & $100 \%$ \\
\hline
\end{tabular}

\begin{tabular}{|l|r|r|}
\hline \multicolumn{3}{|c|}{ Aufteilung nach Straßenzustand } \\
\hline nass/feucht & 3 & $8 \%$ \\
\hline winterglatt & 0 & $0 \%$ \\
\hline schlüpfrig & 0 & $0 \%$ \\
\hline trocken & 33 & $92 \%$ \\
\hline Summe & 36 & $100 \%$ \\
\hline
\end{tabular}

\begin{tabular}{|l|r|r|}
\hline \multicolumn{3}{|c|}{ Aufteilung nach Lichtverhältnissen } \\
\hline dunkel/dämmrig & 2 & $6 \%$ \\
\hline hell & 34 & $94 \%$ \\
\hline Summe & 36 & $100 \%$ \\
\hline
\end{tabular}

\begin{tabular}{|l|r|r|}
\hline \multicolumn{3}{|c|}{ Aufteilung nach LSA-Merkmal } \\
\hline LSA an & 12 & $33 \%$ \\
\hline LSA aus & 0 & $0 \%$ \\
\hline Unfälle insgesamt & 36 & $100 \%$ \\
\hline
\end{tabular}




\begin{tabular}{|l|r|r|}
\hline \multicolumn{3}{|c|}{ Alleinunfall } \\
\hline PTW-Fahrer & 0 & $0 \%$ \\
\hline Unfälle insgesamt & 36 & $100 \%$ \\
\hline
\end{tabular}

\begin{tabular}{|l|r|r|}
\hline \multicolumn{3}{|c|}{ Hauptverursacher } \\
\hline PTW-Fahrer & 28 & $78 \%$ \\
\hline Unfälle insgesamt & 36 & $100 \%$ \\
\hline
\end{tabular}

\begin{tabular}{|l|r|r|}
\hline \multicolumn{3}{|c|}{ Aufteilung nach Wochentag } \\
\hline $\mathbf{1}$ (Sonntag) & 2 & $6 \%$ \\
\hline $\mathbf{2}$ & 1 & $3 \%$ \\
\hline $\mathbf{3}$ & 4 & $11 \%$ \\
\hline $\mathbf{4}$ & 9 & $25 \%$ \\
\hline $\mathbf{5}$ & 6 & $17 \%$ \\
\hline $\mathbf{6}$ & 10 & $28 \%$ \\
\hline $\mathbf{7}$ & 4 & $11 \%$ \\
\hline Summe & 36 & $100 \%$ \\
\hline
\end{tabular}

\begin{tabular}{|l|r|r|}
\hline \multicolumn{3}{|c|}{ Aufteilung nach Monat } \\
\hline $\mathbf{1}$ (Januar) & 0 & $0 \%$ \\
\hline $\mathbf{2}$ & 0 & $0 \%$ \\
\hline $\mathbf{3}$ & 1 & $3 \%$ \\
\hline $\mathbf{4}$ & 5 & $14 \%$ \\
\hline $\mathbf{5}$ & 5 & $14 \%$ \\
\hline $\mathbf{6}$ & 3 & $8 \%$ \\
\hline $\mathbf{7}$ & 3 & $8 \%$ \\
\hline $\mathbf{8}$ & 3 & $8 \%$ \\
\hline $\mathbf{9}$ & 8 & $22 \%$ \\
\hline $\mathbf{1 0}$ & 4 & $11 \%$ \\
\hline $\mathbf{1 1}$ & 2 & $6 \%$ \\
\hline $\mathbf{1 2}$ & 2 & $6 \%$ \\
\hline Summe & 36 & $100 \%$ \\
\hline
\end{tabular}

\begin{tabular}{|l|r|r|}
\hline \multicolumn{3}{|c|}{ Aufteilung nach Zielgruppe (bet. PTW-Fahrer) } \\
\hline Jugendliche & 8 & $22 \%$ \\
\hline Fahranfänger & 9 & $25 \%$ \\
\hline Schlechtwetterunf. & 8 & $22 \%$ \\
\hline Frauen & 5 & $14 \%$ \\
\hline Kraftroller & 3 & $8 \%$ \\
\hline Wiedereinsteiger & & $0 \%$ \\
\hline Senioren & 2 & $6 \%$ \\
\hline PTW-Fahrer insg. & 36 & $100 \%$ \\
\hline
\end{tabular}

\begin{tabular}{|l|r|r|}
\hline \multicolumn{2}{|c|}{ Unfälle auf Streckenabschnitten mit folgenden Eigenschaften: } \\
\hline mindestens 4 Knoten/km & 25 & 9 \\
\hline erhebliche Mängel im Straßenzustand & 14 & $69 \%$ \\
\hline Straße mit Straßenbahn auf der Fahrbahn & 2 & $25 \%$ \\
\hline Querschnitt mit baulich abgesetzter Mitteltrennung & 8 & $39 \%$ \\
\hline mindestens 2 Fahrstreifen pro Richtung & 21 & $6 \%$ \\
\hline lückenhafte oder keine angrenzende Bebauung & 9 & $22 \%$ \\
\hline kreuzende Gleisanlagen & 5 & \\
\hline Parkflächen am Fahrbahnrand & 6 & $58 \%$ \\
\hline zulässige Höchstgeschwindigkeit über 50 km/h & 0 & $25 \%$ \\
\hline zulässige Höchstgeschwindigkeit 30 km/h oder kleiner & 32 & $14 \%$ \\
\hline Fahrbahnrand: Bordstein & $17 \%$ \\
\hline
\end{tabular}


Unfallkonstellation (8): Unfall innerorts an Kreuzungen, Einmündungen oder ohne Charakteristik mit in gleicher Richtung verkehrenden $\mathrm{Kfz}$

\begin{tabular}{|l|r|r|}
\hline \multicolumn{3}{|c|}{ Aufteilung nach Unfallart } \\
\hline Art 1 & 0 & $0 \%$ \\
\hline Art 2 & 0 & $0 \%$ \\
\hline Art 3 & 35 & $100 \%$ \\
\hline Art 4 & 0 & $0 \%$ \\
\hline Art 5 & 0 & $0 \%$ \\
\hline Art 6 & 0 & $0 \%$ \\
\hline Art 7 & 0 & $0 \%$ \\
\hline Art 8 & 0 & $0 \%$ \\
\hline Art 9 & 0 & $0 \%$ \\
\hline Art 0 & 0 & $0 \%$ \\
\hline Summe & 35 & $100 \%$ \\
\hline
\end{tabular}

\begin{tabular}{|l|r|r|}
\hline \multicolumn{3}{|c|}{ Aufteilung nach Unfallschwere } \\
\hline $\mathbf{U}(\mathrm{GT})$ & 0 & $0 \%$ \\
\hline $\mathbf{U}(\mathrm{SV})$ & 2 & $6 \%$ \\
\hline $\mathbf{U}(\mathrm{LV})$ & 7 & $20 \%$ \\
\hline $\mathbf{U}(\mathbf{S})$ & 26 & $74 \%$ \\
\hline Summe & 35 & $100 \%$ \\
\hline
\end{tabular}

\begin{tabular}{|l|r|r|}
\hline \multicolumn{3}{|c|}{ Aufteilung nach Straßenzustand } \\
\hline nass/feucht & 5 & $14 \%$ \\
\hline winterglatt & 0 & $0 \%$ \\
\hline schlüpfrig & 0 & $0 \%$ \\
\hline trocken & 30 & $86 \%$ \\
\hline Summe & 35 & $100 \%$ \\
\hline
\end{tabular}

\begin{tabular}{|l|r|r|}
\hline \multicolumn{3}{|c|}{ Aufteilung nach Lichtverhältnissen } \\
\hline dunkel/dämmrig & 5 & $14 \%$ \\
\hline hell & 25 & $71 \%$ \\
\hline Summe & 35 & $100 \%$ \\
\hline
\end{tabular}

\begin{tabular}{|l|r|r|}
\hline \multicolumn{3}{|c|}{ Aufteilung nach LSA-Merkmal } \\
\hline LSA an & 13 & $37 \%$ \\
\hline LSA aus & 0 & $0 \%$ \\
\hline Unfälle insgesamt & 35 & $100 \%$ \\
\hline
\end{tabular}

\begin{tabular}{|l|r|l|}
\hline \multicolumn{3}{|c|}{ Aufteilung nach Aufprall auf Hinderniss } \\
\hline 0 (Baum) & nicht untersucht & \\
\hline $\mathbf{1}$ (Mast) & nicht untersucht & \\
\hline 2 (Widerlager) & nicht untersucht & \\
\hline 3 (Schutzplanke) & nicht untersucht & \\
\hline 4 (sonst. Hindernis) & nicht untersucht & \\
\hline 5 (kein Hindernis) & nicht untersucht & \\
\hline Unfälle insgesamt & & \\
\hline
\end{tabular}

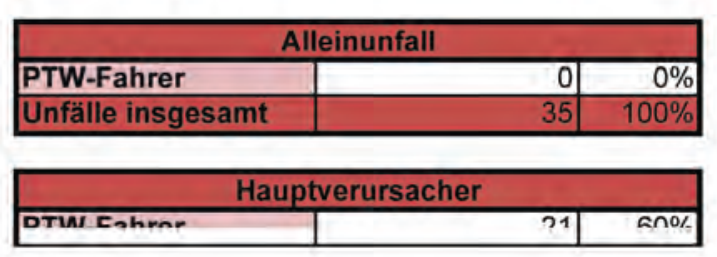




\begin{tabular}{|l|r|r|}
\hline \multicolumn{3}{|c|}{ Aufteilung nach Wochentag } \\
\hline $\mathbf{1}$ (Sonntag) & 3 & $9 \%$ \\
\hline $\mathbf{2}$ & 5 & $14 \%$ \\
\hline $\mathbf{3}$ & 6 & $17 \%$ \\
\hline $\mathbf{4}$ & 7 & $20 \%$ \\
\hline $\mathbf{5}$ & 5 & $14 \%$ \\
\hline $\mathbf{6}$ & $\mathbf{4}$ & $11 \%$ \\
\hline $\mathbf{7}$ & 5 & $14 \%$ \\
\hline Summe & 35 & $100 \%$ \\
\hline
\end{tabular}

\begin{tabular}{|l|r|r|}
\hline \multicolumn{3}{|c|}{ Aufteilung nach Monat } \\
\hline $\mathbf{1}$ (Januar) & 0 & $0 \%$ \\
\hline $\mathbf{2}$ & 0 & $0 \%$ \\
\hline $\mathbf{3}$ & 2 & $6 \%$ \\
\hline $\mathbf{4}$ & 5 & $14 \%$ \\
\hline $\mathbf{5}$ & 4 & $11 \%$ \\
\hline $\mathbf{6}$ & 6 & $17 \%$ \\
\hline $\mathbf{7}$ & 2 & $6 \%$ \\
\hline $\mathbf{8}$ & 2 & $6 \%$ \\
\hline $\mathbf{9}$ & 7 & $20 \%$ \\
\hline $\mathbf{1 0}$ & 5 & $14 \%$ \\
\hline $\mathbf{1 1}$ & 2 & $6 \%$ \\
\hline $\mathbf{1 2}$ & 0 & $0 \%$ \\
\hline Summe & 35 & $100 \%$ \\
\hline
\end{tabular}

\begin{tabular}{|l|r|r|}
\hline \multicolumn{3}{|c|}{ Aufteilung nach Zielgruppe (bet. PTW-Fahrer) } \\
\hline Jugendliche & 6 & $17 \%$ \\
\hline Fahranfänger & 4 & $11 \%$ \\
\hline Schlechtwetterunf. & 9 & $25 \%$ \\
\hline Frauen & 1 & $3 \%$ \\
\hline Kraftroller & 3 & $8 \%$ \\
\hline Wiedereinsteiger & & $0 \%$ \\
\hline Senioren & 3 & $8 \%$ \\
\hline PTW-Fahrer insg. & 36 & $100 \%$ \\
\hline
\end{tabular}

\begin{tabular}{|l|r|r|}
\hline \multicolumn{2}{|c|}{ Unfälle auf Streckenabschnitten mit folgenden Eigenschaften: } \\
\hline mindestens 4 Knoten/km & 17 & $49 \%$ \\
\hline erhebliche Mängel im Straßenzustand & 10 & $29 \%$ \\
\hline Straße mit Straßenbahn auf der Fahrbahn & 8 & $23 \%$ \\
\hline Querschnitt mit baulich abgesetzter Mitteltrennung & 3 & $9 \%$ \\
\hline mindestens 2 Fahrstreifen pro Richtung & 9 & $26 \%$ \\
\hline lückenhafte oder keine angrenzende Bebauung & 31 & $89 \%$ \\
\hline kreuzende Gleisanlagen & 15 & $43 \%$ \\
\hline Parkflächen am Fahrbahnrand & 0 & $0 \%$ \\
\hline Zulässige Höchstgeschwindigkeit über 50 km/h & 3 & $9 \%$ \\
\hline Zulässige Höchstgeschwindigkeit 30 km/h oder kleiner & 0 & $0 \%$ \\
\hline Fahrbahnrand; Bordstein & 31 & $89 \%$ \\
\hline Unfälle insgesamt & 35 & $100 \%$ \\
\hline
\end{tabular}


Unfallkonstellation (9): Fahrunfall innerorts anderer Art ohne Charakteristik

\begin{tabular}{|l|r|r|}
\hline \multicolumn{3}{|c|}{ Aufteilung nach Unfallart } \\
\hline Art 1 & 0 & $0 \%$ \\
\hline Art 2 & 0 & $0 \%$ \\
\hline Art 3 & 0 & $0 \%$ \\
\hline Art 4 & 0 & $0 \%$ \\
\hline Art 5 & 0 & $0 \%$ \\
\hline Art 6 & 0 & $0 \%$ \\
\hline Art 7 & 0 & $0 \%$ \\
\hline Art 8 & 0 & $0 \%$ \\
\hline Art 9 & 0 & $0 \%$ \\
\hline Art 0 & 12 & $100 \%$ \\
\hline Summe & 12 & $100 \%$ \\
\hline
\end{tabular}

\begin{tabular}{|l|r|r|}
\hline \multicolumn{3}{|c|}{ Aufteilung nach Unfallschwere } \\
\hline $\mathbf{U}(\mathrm{GT})$ & 0 & $0 \%$ \\
\hline $\mathbf{U}(\mathrm{SV})$ & 5 & $42 \%$ \\
\hline $\mathbf{U}(\mathrm{LV})$ & 6 & $50 \%$ \\
\hline $\mathbf{U}(\mathrm{S})$ & 1 & $8 \%$ \\
\hline Summe & 12 & $100 \%$ \\
\hline
\end{tabular}

\begin{tabular}{|l|r|r|}
\hline \multicolumn{3}{|c|}{ Aufteilung nach Straßenzustand } \\
\hline nass/feucht & 7 & $58 \%$ \\
\hline winterglatt & 1 & $8 \%$ \\
\hline schlüpfrig & 1 & $8 \%$ \\
\hline trocken & 3 & $25 \%$ \\
\hline Summe & 12 & $100 \%$ \\
\hline
\end{tabular}

\begin{tabular}{|l|r|r|}
\hline \multicolumn{3}{|c|}{ Aufteilung nach Lichtverhältnissen } \\
\hline dunkel/dämmrig & 3 & $25 \%$ \\
\hline hell & 9 & $75 \%$ \\
\hline Summe & 12 & $100 \%$ \\
\hline
\end{tabular}

\begin{tabular}{|l|r|r|}
\hline \multicolumn{3}{|c|}{ Aufteilung nach LSA-Merkmal } \\
\hline LSA an & $0 \%$ \\
\hline LSA aus & 12 & $0 \%$ \\
\hline Unfälle insgesamt & $100 \%$ \\
\hline
\end{tabular}

\begin{tabular}{|l|r|r|}
\hline \multicolumn{3}{|c|}{ Alleinunfall } \\
\hline PTW-Fahrer & 12 & $100 \%$ \\
\hline Unfälle insgesamt & 12 & $100 \%$ \\
\hline
\end{tabular}

\begin{tabular}{|l|r|r|}
\hline \multicolumn{3}{|c|}{ Hauptverursacher } \\
\hline PTW-Fahrer & 12 & $100 \%$ \\
\hline Unfälle insgesamt & 12 & $100 \%$ \\
\hline
\end{tabular}

\begin{tabular}{|l|r|r|}
\hline \multicolumn{3}{|c|}{ Aufteilung nach Wochentag } \\
\hline $\mathbf{1}$ (Sonntag) & 1 & $8 \%$ \\
\hline $\mathbf{2}$ & 2 & $17 \%$ \\
\hline 3 & 3 & $25 \%$ \\
\hline 4 & 0 & $0 \%$ \\
\hline 5 & 3 & $25 \%$ \\
\hline 6 & 2 & $17 \%$ \\
\hline- & & $\cdots$ \\
\hline
\end{tabular}




\begin{tabular}{|l|r|r|}
\hline \multicolumn{3}{|c|}{ Aufteilung nach Monat } \\
\hline 1 (Januar) & 0 & $0 \%$ \\
\hline 2 & 0 & $0 \%$ \\
\hline 3 & 0 & $0 \%$ \\
\hline 4 & 1 & $8 \%$ \\
\hline 5 & 2 & $17 \%$ \\
\hline 6 & 1 & $8 \%$ \\
\hline 7 & 0 & $0 \%$ \\
\hline 8 & 3 & $25 \%$ \\
\hline 9 & 1 & $8 \%$ \\
\hline 10 & 3 & $25 \%$ \\
\hline 11 & 0 & $0 \%$ \\
\hline 12 & 1 & $8 \%$ \\
\hline Summe & 12 & $100 \%$ \\
\hline
\end{tabular}

\begin{tabular}{|l|r|r|}
\hline \multicolumn{3}{|c|}{ Aufteilung nach Zielgruppe (bet. PTW-Fahrer) } \\
\hline Jugendliche & 0 & $0 \%$ \\
\hline Fahranfänger & 1 & $8 \%$ \\
\hline Schlechtwetterunf. & 8 & $67 \%$ \\
\hline Frauen & 1 & $8 \%$ \\
\hline Kraftroller & 1 & $8 \%$ \\
\hline Wiedereinsteiger & 1 & $0 \%$ \\
\hline Senioren & 1 & $8 \%$ \\
\hline PTW-Fahrer insg. & 12 & $100 \%$ \\
\hline
\end{tabular}

\section{Unfälle auf Streckenabschnitten mit folgenden Eigenschaften:}

mindestens $4 \mathrm{Knoten} / \mathrm{km}$

erhebliche Mängel im Straßenzustand

Straße mit Straßenbahn auf der Fahrbahn

Querschnitt mit baulich abgesetzter Mitteltrennung

mindestens 2 Fahrstreifen pro Richtung

lückenhafte oder keine angrenzende Bebauung

kreuzende Gleisanlagen

Parkflächen am Fahrbahnrand

zulässige Höchstgeschwindigkeit über $50 \mathrm{~km} / \mathrm{h}$

zulässige Höchstgeschwindigkeit $30 \mathrm{~km} / \mathrm{h}$ oder kleiner

Fahrbahnrand: Bordstein

Unfälle insgesamt

\begin{tabular}{r|r|}
\hline 6 & $50 \%$ \\
\hline 3 & $25 \%$ \\
\hline 6 & $50 \%$ \\
\hline 0 & $0 \%$ \\
\hline 1 & $8 \%$ \\
\hline 8 & $67 \%$ \\
\hline 5 & $42 \%$ \\
\hline 0 & $0 \%$ \\
\hline 2 & $17 \%$ \\
\hline 0 & $0 \%$ \\
\hline 10 & $83 \%$ \\
\hline 12 & $100 \%$ \\
\hline
\end{tabular}


Unfallkonstellation (10): Auffahrunfall innerorts ohne Charakteristik

\begin{tabular}{|l|r|r|}
\hline \multicolumn{3}{|c|}{ Aufteilung nach Unfallart } \\
\hline Art 1 & 0 & $0 \%$ \\
\hline Art 2 & 24 & $100 \%$ \\
\hline Art 3 & 0 & $0 \%$ \\
\hline Art 4 & 0 & $0 \%$ \\
\hline Art 5 & 0 & $0 \%$ \\
\hline Art 6 & 0 & $0 \%$ \\
\hline Art 7 & 0 & $0 \%$ \\
\hline Art 8 & 0 & $0 \%$ \\
\hline Art 9 & 0 & $0 \%$ \\
\hline Art 0 & 0 & $0 \%$ \\
\hline Summe & 24 & $100 \%$ \\
\hline
\end{tabular}

\begin{tabular}{|l|r|r|}
\hline \multicolumn{3}{|c|}{ Aufteilung nach Unfallschwere } \\
\hline $\mathbf{U}(\mathrm{GT})$ & 0 & $0 \%$ \\
\hline $\mathbf{U}(\mathrm{SV})$ & 1 & $4 \%$ \\
\hline $\mathbf{U}(\mathrm{LV})$ & 7 & $29 \%$ \\
\hline $\mathbf{U}$ (S) & 16 & $67 \%$ \\
\hline Summe & 24 & $100 \%$ \\
\hline
\end{tabular}

\begin{tabular}{|l|r|r|}
\hline \multicolumn{3}{|c|}{ Aufteilung nach Straßenzustand } \\
\hline nass/feucht & 4 & $17 \%$ \\
\hline winterglatt & 0 & $0 \%$ \\
\hline Schlüpfrig & 0 & $0 \%$ \\
\hline trocken & 20 & $83 \%$ \\
\hline Summe & 24 & $100 \%$ \\
\hline
\end{tabular}

\begin{tabular}{|l|r|r|}
\hline \multicolumn{3}{|c|}{ Aufteilung nach Lichtverhältnissen } \\
\hline dunkel/dämmrig & 2 & $8 \%$ \\
\hline hell & 22 & $92 \%$ \\
\hline Summe & 24 & $100 \%$ \\
\hline
\end{tabular}

\begin{tabular}{|l|r|r|}
\hline \multicolumn{3}{|c|}{ Aufteilung nach LSA-Merkmal } \\
\hline LSA an & 9 & $38 \%$ \\
\hline LSA aus & 0 & $0 \%$ \\
\hline Unfälle insgesamt & 24 & $100 \%$ \\
\hline
\end{tabular}

\begin{tabular}{|l|r|r|}
\hline \multicolumn{3}{|c|}{ Alleinunfall } \\
\hline PTW-Fahrer & 0 & $0 \%$ \\
\hline Unfälle insgesamt & 24 & $100 \%$ \\
\hline
\end{tabular}

\begin{tabular}{|l|r|r|}
\hline \multicolumn{3}{|c|}{ Hauptverursacher } \\
\hline PTW-Fahrer & 15 & $63 \%$ \\
\hline Unfälle insgesamt & 24 & $100 \%$ \\
\hline
\end{tabular}

\begin{tabular}{|l|r|r|}
\hline \multicolumn{3}{|c|}{ Aufteilung nach Wochentag } \\
\hline $\mathbf{1}$ (Sonntag) & 1 & $4 \%$ \\
\hline $\mathbf{2}$ & 4 & $17 \%$ \\
\hline $\mathbf{3}$ & 4 & $17 \%$ \\
\hline $\mathbf{4}$ & 5 & $21 \%$ \\
\hline $\mathbf{5}$ & 4 & $17 \%$ \\
\hline $\mathbf{6}$ & 3 & $13 \%$ \\
\hline $\mathbf{7}$ & 3 & $13 \%$ \\
\hline Summe & 24 & $100 \%$ \\
\hline
\end{tabular}




\begin{tabular}{|l|r|r|}
\hline \multicolumn{3}{|c|}{ Aufteilung nach Monat } \\
\hline 1 (Januar) & 0 & $0 \%$ \\
\hline 2 & 0 & $0 \%$ \\
\hline 3 & 0 & $0 \%$ \\
\hline 4 & 2 & $8 \%$ \\
\hline 5 & 1 & $4 \%$ \\
\hline 6 & 3 & $13 \%$ \\
\hline 7 & 3 & $13 \%$ \\
\hline 8 & 4 & $17 \%$ \\
\hline 9 & 3 & $13 \%$ \\
\hline 10 & 6 & $25 \%$ \\
\hline 11 & 1 & $4 \%$ \\
\hline 12 & 1 & $4 \%$ \\
\hline
\end{tabular}

\begin{tabular}{|l|r|r|}
\hline \multicolumn{2}{|c|}{ Aufteilung nach Zielgruppe (bet. PTW-Fahrer) } \\
\hline Jugendliche & 9 & $38 \%$ \\
\hline Fahranfänger & 3 & $13 \%$ \\
\hline Schlechtwetterunf. & 6 & $25 \%$ \\
\hline Frauen & 2 & $8 \%$ \\
\hline Kraftroller & 0 & $0 \%$ \\
\hline Wiedereinsteiger & & $0 \%$ \\
\hline Senioren & 1 & $4 \%$ \\
\hline PTW-Fahrer insg. & 24 & $100 \%$ \\
\hline
\end{tabular}

\begin{tabular}{|l|r|r|}
\hline \multicolumn{2}{|c|}{ Unfälle auf Streckenabschnitten mit folgenden Eigenschaften: } \\
\hline mindestens 4 Knoten/km & 14 & $58 \%$ \\
\hline erhebliche Mängel im Straßenzustand & 7 & $29 \%$ \\
\hline Straße mit Straßenbahn auf der Fahrbahn & 8 & $33 \%$ \\
\hline Querschnitt mit baulich abgesetzter Mitteltrennung & 1 & $4 \%$ \\
\hline mindestens 2 Fahrstreifen pro Richtung & 4 & $17 \%$ \\
\hline lückenhafte oder keine angrenzende Bebauung & 17 & $71 \%$ \\
\hline kreuzende Gleisanlagen & 8 & $33 \%$ \\
\hline Parkflächen am Fahrbahnrand & 4 & $17 \%$ \\
\hline Zulässige Höchstgeschwindigkeit über 50 km/h & 2 & $8 \%$ \\
\hline zulässige Höchstgeschwindigkeit 30 km/h oder kleiner & 0 & $0 \%$ \\
\hline Fahrbahnrand: Bordstein & 21 & $88 \%$ \\
\hline Unfälle insgesamt & 24 & $100 \%$ \\
\hline
\end{tabular}


Anlage 09: Fragebogen zur Befragung in der Zeitschrift „MOTORRAD“

Fragebogen zur Motorradsicherheit

1 Angaben zur eigenen Maschine und zum Motorradfahren selbst

1.1 Was macht für Sie die Faszination des Motorradfahrens aus?

\begin{tabular}{|c|c|c|c|}
\hline I ja & Heschleunigung & Tle & Getuhl der t reihert \\
\hline$[\mathrm{lb}$ & Geschwindigkent & $1 \mathrm{Ht}$ & Wortell 2-Rad gegenuber 4Rad (Parken, ..Uurchschlangeln im Stau" etc.) \\
\hline 110 & Sportichkent & 119 & Kostemvortell 2-Rad gegenuber 4 Rad \\
\hline I I0 & Kunventanit & {[]$^{\mathrm{h}}$} & Sonstiges \\
\hline
\end{tabular}

1.2 Welches Motorrad besitzen Sie? Dei wetweren Motorraderm obs, wit de on Sie am weister fahrenj Hersteller:_ Typ:___ Kaufdatum:

1.3 Entspricht das Motorrad der Serie oder haben Sie es modifiziert? [ ] a serienmähig [ ]b modifiziert

1.4 Wenn modifiziert, was haben Sie an Ihrem Motorrad verändert?

1.5 We viel Geld geben Sie ungefähr im Jahr für Teile und Modifi kationen am Motorrad aus? Euro

1.6 We viele Kilometer fahren Sie mit dem Motorrad durchschnittlich im Jahr?
[ ] u unter $3000 \mathrm{~km}$
[ ] 3000 bis $4000 \mathrm{~km}$
[ ]c 4000 bis $5000 \mathrm{lom}$ [ ]d mehr als $5000 \mathrm{lom}$

1.7 We verteilt sich etwa Ihre jährliche Fahrleistung ortsbezogen? (fr Probent, gesoliatzt) inoerorts aukerorts ohne Autobahn Autobahn

1.8 Zu welchen Jahreszeiten nutzen Sie lhr Motorrad hauptsächlich?
[ ] a Frihling
[ ]b Sommer
[ ]c Herbst
[ ]d Winter

1.9 We nutzen Sie lhr Motorrad hauptsächlich?
[ ] 3 Freizeit
[ ] B Altag
[ ]c beides

1.10 Fahren Sie mit Ihrem Motorrad auch bei sohlechtem Wetter? (hass, hall, glatt, Regen, So/mee...) [ ]a (praktisch) immer (ceine Rücksicht aư das Wietter)
[ ] ]b häutig
[ ]c gelegentlioh
I ]d selten
I le (praktisch) nie

$1.11 \ldots$ und im Winter?

I ]a (praktisch)mmer I ]b häufig [ ]o gelegentlich I ]d sehten [ ]e (praktisch) nie

[ ]f in Wirter ist es abgemeldet (Saisonkennzeichen)

1.12 Wie schätzen Sie sich als Motorradfahrer ein?

Anfänger [ ]0 [ ]1 [ ]2 [ ]3 [ ]4 erfahrener und geübter Fahrer

1.13 We schätzen Sie lhren Fahrstil mit dem Motorrad ein? sportlich [ ]0 [ ]1 [ ]2 [ ]3 [ ]4 bedacht

Abbildung 0-1: Fragebogen Seite 1 
1.14 Haben Sie schon einmal an einem Motorrad-Fahrscherheitstraining teilgenommen?
[ ]a nein
I jb ja,
mal
[ ]c jährlich

1.15 Fahren Siehäufiger allein oder in der Gruppe?
[ ] a häufiger allein
[ ]b häufiger in der Gruppe
[ ]c beides etwa gleich häutig

1.16 We häufig nehmen Sie jemanden auf dem Motorrad mit?
[ ]a (praictisoh) inmer
[ ]b häustig
[ ]c gelegentlich
[ ]d selten [ ]e (praktisch) nie

2 Verstoß gegen Verkehrsregeh

2.1 Sind Sie schon einmal wegen eines Verstoßes gegen die Ver kehrsregeln mit Ihrem Motorrad belangt worden?

[ ] a nein (weiter mt Pht. 3)

[ ] $\mathrm{b} \mathrm{ja} \rightarrow$ wie oft in den letzten 3 Jahren? mal

2.2 Wenn ja, welcher Art waren diese Verstöße? (Wh den ietzten dine Jahwen)

\begin{tabular}{|l|l|}
\hline Art des Verstokes & Bestroiung (Bu kgeld, Punkte, Führerscheinentzug etc.) \\
\hline & \\
\hline & \\
\hline & \\
\hline & \\
\hline & \\
\hline
\end{tabular}

\section{Verkehrstufall}

3.1 Hatten Sie mit threm Motorrad jemals einen Verkehrsunfall?
I ] nein (weiter mit Pld, 4)
[ ]b ja $\rightarrow$ vine riele? mal

3.2 Bitte beschreiben Sie den Unfall, der Ihnen am besten in Erinnerung ist! (in kuzen Stichmorten)

3.3 Wann war der Unfall?

3.4 Wurde der Unfall aufgenommen?
[ ] nein
[ ]b ja $\rightarrow$ Won wem?

3.5 Waren weitere Verkehrsteilnehmer an dem Unfall beteiligt?
[ ] ] Pkw
[ ] to Lkw/Lastzug
[] ]e Bus
I ]d Kaitrad
[ ]e Fahrrad
[ ]f Fukgänger
[ ]g Sonstige
[ ] th keine

3.6 We viele Ver kehrsteilnehmer waren außer Ihnen noch beteiligt?
[ ]a Ener
[ ] Z Zwei
[ ]o Drei
[ ]d mehr als drei
[ ]e keiner

Abbildung 0-2: Fragebogen Seite 2 
3.7 Was war die Haupturssohe für diesen Unfall? (Mehachnenumgen möghth)

\begin{tabular}{|c|c|c|c|}
\hline [ ] & ioh wurde ubersehen & {$[\mathrm{l}$} & Selbstubersohatzung won mir \\
\hline [ ]o & $\begin{array}{l}\text { melne bieschimindigkert munde takoh } \\
\text { eingeschätzt }\end{array}$ & [ ] & Dnaohtsamkert von mir \\
\hline [ Je & $\begin{array}{l}\text { teohinisohe wangel am r ahredug des } \\
\text { Unfallgegners }\end{array}$ & I It & technisohe wengel am Wotomad \\
\hline [ ] & sohleohiter rahitahinustand & [ ] & ubertionte lieschuluidigkent des Untallgegners \\
\hline$[$ ] & ungunstige douttenungsuehtaltnitse & III & uberhohte beschulindigket won mir \\
\hline I Jk: & unzurelchende Schtuentatnisse & {$[\mathrm{l}$} & Sonstiges. \\
\hline
\end{tabular}

3.8 Wie waren die Gegebenheiten am Unf

\begin{tabular}{|c|c|c|c|c|c|c|}
\hline Drtslage & []$]$ & innerorts & {$[\mathrm{b} b$} & aukerorts, Autobahn & [ ]0 & aukerorts, Landstrake \\
\hline Freie Strecke & {$[$ ] $]$} & Gerade & {$[\mathrm{b}$} & weite Kurve & [ ]o & enge Kunve \\
\hline oder Krotenpunkt & {$[$ ] $]$} & Enmiündung & {$[\mathrm{b} b$} & Kreuzung & {$[\mathrm{]c}$} & Keisuerkehr \\
\hline mit Werkehrseregelung & {$[$ ] $]$} & Reohts vor Links & {$[\mathrm{b}$} & mit Ampel & [ ]o & jə, ohne Ampel \\
\hline \multirow{4}{*}{$\begin{array}{l}\text { Won der rahitiahin } \\
\text { abigekommen und } \\
\text { Aufprall auf Hindemis }\end{array}$} & {$[$ ] $]$} & nein & {$[\mathrm{b}$} & ja, 'ilielohe? & [ ] & Baum \\
\hline & & & & & [ ] & Schutzplanke \\
\hline & & & & & [ ] & West \\
\hline & & & & & [ ] & Sonstiges \\
\hline Fahıыahnzustand & {$[$ ] $]$} & trocken & {$[\mathrm{b} b$} & nass & {$[$ ]o } & winterglatt \\
\hline Lichtuertiältrisse & []$]$ & Tageslicht & {$[\mathrm{b}$} & Dämıтеกıng & [ ]o & Dunkelheit \\
\hline
\end{tabular}

3.9 Sind Sie tei dem Unfall verletzt worden?

[ ] п пеiп

[ ]b ja, leicht (ambulante Behandlung oder Kankenhausaufenthat weriger als 24 h)

[ ]c ja, sohwer (staionäre Behandlung, Kankenhausaufenthalt mehr as 24 h)

3.10 Wenn ja, wro waren Ihre Verletzungen?

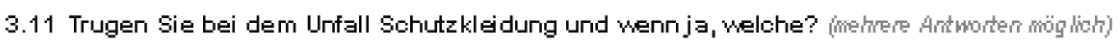
[ ]
[ ] J Jacke
[ ]0 Jacke mit Protektoren
[ ]g Nierengurt
[ ]d Hose
[ ]h Motorradhandschuhe
[ ]e Hose mit Protektoren
[ ] ] Wotoradstiefel
[ ] k sonstiges
[ li keine sohutzkleidung

[ ]f 'illihtelsäulenschustz' Riückenprotedtor

3.12 Mit welchem Motorrad ist diezer Unfall pissiert?
[ ]a wie oben beschineben
[ ] b anderes botomad $\rightarrow$ Hersteller BTyp?

Abbildung 0-3: Fragebogen Seite 3 
3.13 Hatte das Motorrad ABS?

[ ] a ja

[ ]b nein

3.14 We hoch war der Schaden am Motorrad?

Euro [ ]a Totalschaden

3.15 Ab wann sind Sie nach dem Unfall wieder Motorrad gefahren? (7age. Mocheri oder Monate!

[ ] a bin danach nioht mehr Motorrad gefahren

3.16 Haben Sie sich nach dem Unfall wieder ein neues Motorrad gekaut?
[ ]a nein
[ ] b ja $\rightarrow$ Wann (Tage, Wochen oder Monate'?
$\rightarrow$ Mit anderer Ausstattung?

[ ]c ich konnte das gleiche Motorrad weiter benutzen

3.17 Sind weitere beteiligte Personen verletzt worden? (mehvere Andwortern farb, s und d anöglioh)

[ ] a nein

[ ]b ja, leicht (ambulante Behandlung oder Krankenhausaufenthat weniger als $24 \mathrm{~h}$ )

Anzahi;

[ ]c ja، schwer (staionäre Behandlung, Kankenhausaufenthalt mehr ak $24 \mathrm{~h}$ )

Anzahil:

[ ] ja, geötet

Anzahl

4 Persönliche Angaben

Atter: Jahre

Geschlecht:

[ ]a männlich

[ ]b weiblich

Wohnort (PLZ + Qrt)

Beruf:

Motorradführerschein seit (Sahr)

infedereinsteiger (Jahr):

\section{Abschluss}

5.1 Gibt es zum Thema Motorradfahren und Motorradurfälle weitere Dinge, die im Fragebogen nicht aufgetaucht sind, die Sie uns aber gerne mitteilen möchten?

Abbildung 0-4: Fragebogen Seite 4 


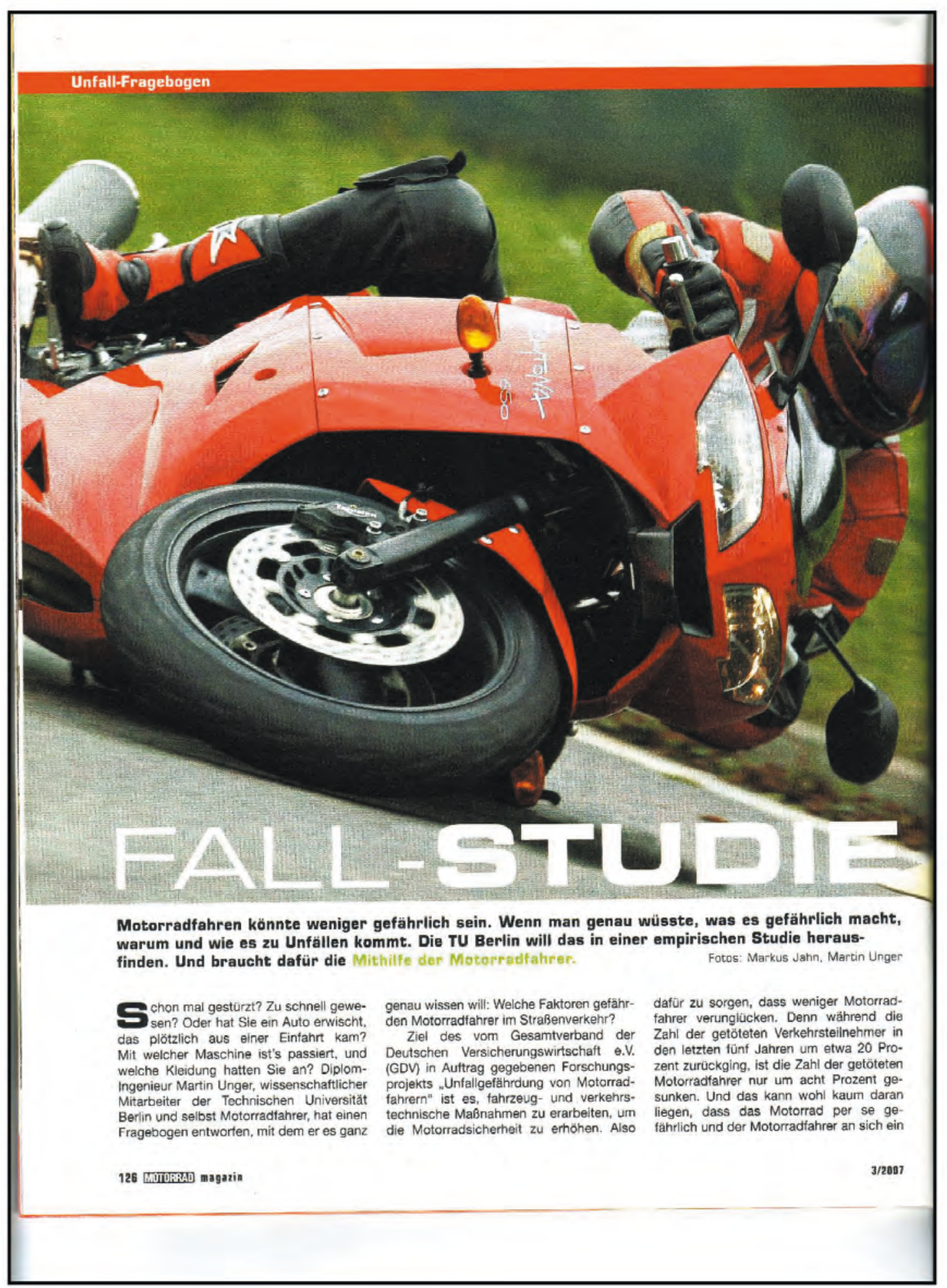

Abbildung 0-5: Artikel Motorrad 03/07 Seite 126 


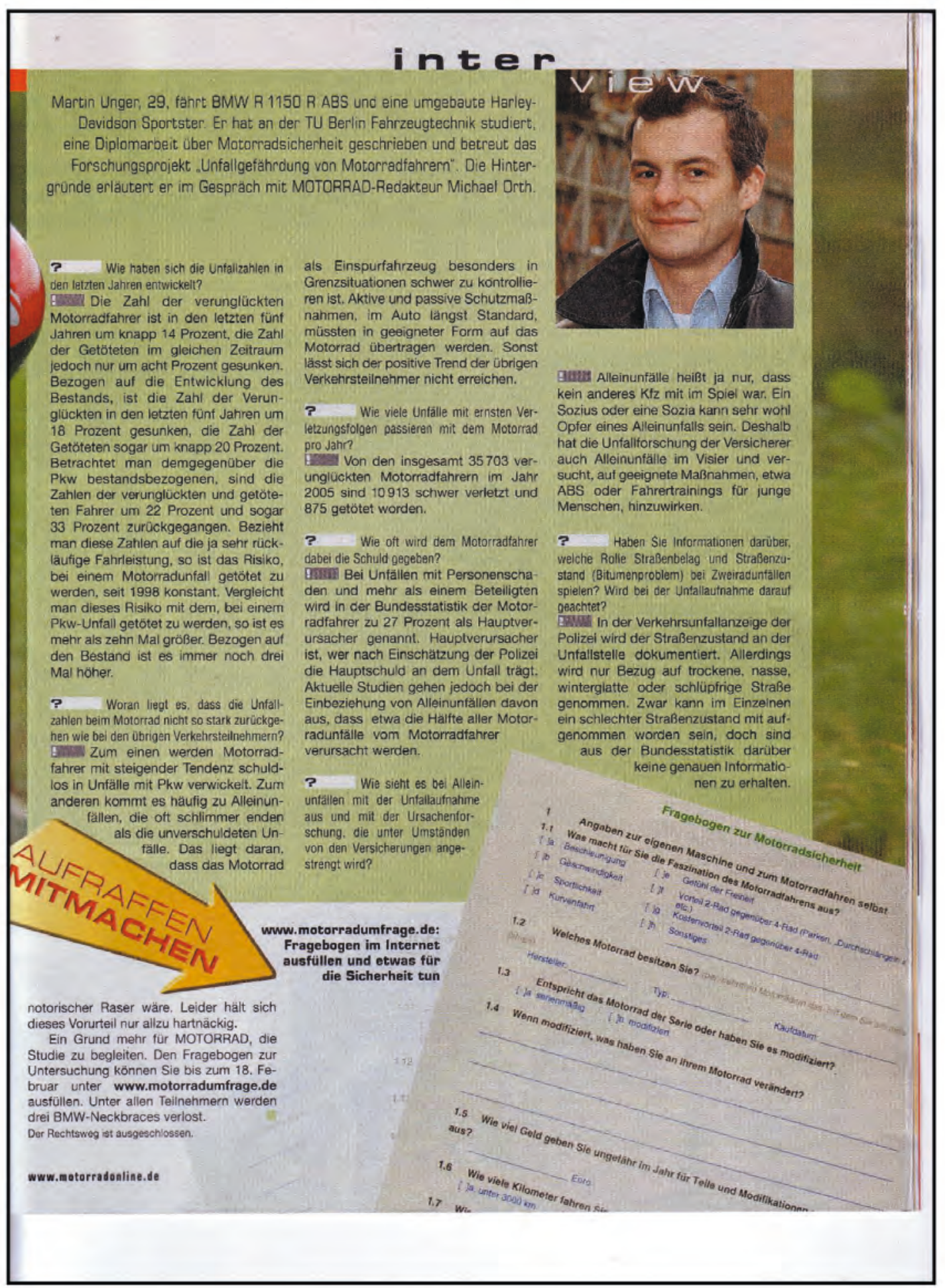

Abbildung 0-6: Artikel Motorrad 03/07 Seite 127 


\section{Anlage 10: Beschreibung der 10 Unfallarten}

Die Unfallart beschreibt vom gesamten Unfallablauf die Bewegungsrichtung der beteiligten Fahrzeuge zueinander beim ersten Zusammensto $B$ auf der Fahrbahn oder, wenn es nicht zum Zusammenstoß gekommen ist, die erste mechanische Einwirkung auf einen Verkehrsteilnehmer. Es werden folgende 10 Unfallarten unterschieden:

1. Zusammenstoß mit anderem Fahrzeug, das anfährt, anhält oder im ruhenden Verkehr steht

Anfahren oder Anhalten ist hier im Zusammenhang mit einer gewollten Fahrtunterbrechung zu sehen, die nicht durch die Verkehrslage veranlasst ist. Ruhender Verkehr im Sinne dieser Unfallart ist das Halten oder Parken am Fahrbahnrand, auf Seitenstreifen, auf den markierten Parkstellen unmittelbar am Fahrbahnrand, auf Gehwegen

oder auf Parkplätzen. Der Verkehr von und zu Parkplätzen mit eigenen Zufahrten gehört zur Unfallart 5.

2. Zusammenstoß mit anderem Fahrzeug, das vorausfährt oder wartet

Unfälle durch Auffahren auf ein Fahrzeug, das selbst noch fuhr oder verkehrsbedingt hielt. Auffahren auf anfahrende bzw. anhaltende Fahrzeuge gehören zur Unfallart 1.

3. Zusammenstoß mit anderem Fahrzeug, das seitlich in gleicher Richtung fährt

Unfälle beim Nebeneinanderfahren (Streifen) oder beim Fahrstreifenwechsel (Schneiden).

4. Zusammenstoß mit anderem Fahrzeug, das entgegenkommt

Zusammenstöße im Begegnungsverkehr, ohne dass ein Kollisionspartner die Absicht hatte, uber die Gegenspur abzubiegen.
5. Zusammenstoß mit anderem Fahrzeug, das einbiegt oder kreuzt

Zu dieser Unfallart gehören Zusammenstöße mit dem Querverkehr und Kollisionen mit Fahrzeugen die aus anderen Straßen, Wegen oder Grundstücken ein biegen oder dorthin abbiegen wollen. Das Auffahren auf wartende Abbieger gehört zur Unfallart 2.

6. Zusammenstoß zwischen Fahrzeug und Fußgänger

Personen, die sich arbeitsbedingt auf der Fahrbahn aufhalten oder noch in enger Verbindung zu einem Fahrzeug stehen, wie Straßenarbeiter, Polizeibeamte bei der Verkehrsregelung oder ausgestiegene Fahrzeuginsassen bei Pannen zählen nicht als Fußgänger. Zusammenstöße mit ihnen gehören zur Unfallart 10.

\section{Aufprall auf ein Hindernis auf der Fahrbahn}

Zu den Hindernissen zählen z.B. umgestürzte Bäume, Steine, verlorene Fracht sowie freilaufende Tiere oder Wild. Zusammenstöße mit geführten Tieren oder Reitern gehören zur Unfallart 10.

\section{8./9. Abkommen von der Fahrbahn nach rechts/links}

Bei diesen Unfallarten ist es nicht zu einem Zusammensto B mit anderen Verkehrsteilnehmern gekommen. Es kann jedoch weitere Unfallbeteiligte geben, z.B, wenn das verunglückte Fahrzeug von der Straße abgekommen ist, weil es einem anderen Verkehrstellnehmer ausgewichen ist, ohne ihn zu berühren.

\section{Unfall anderer Art}

Hier werden alle Unfälle erfasst, die sich nicht einer der Unfallarten von 1 bis 9 zuordnen lassen. 
Anlage 11: Unfallaufnahme in Abhängigkeit der Unfallschwere und der Anzahl der Beteiligten
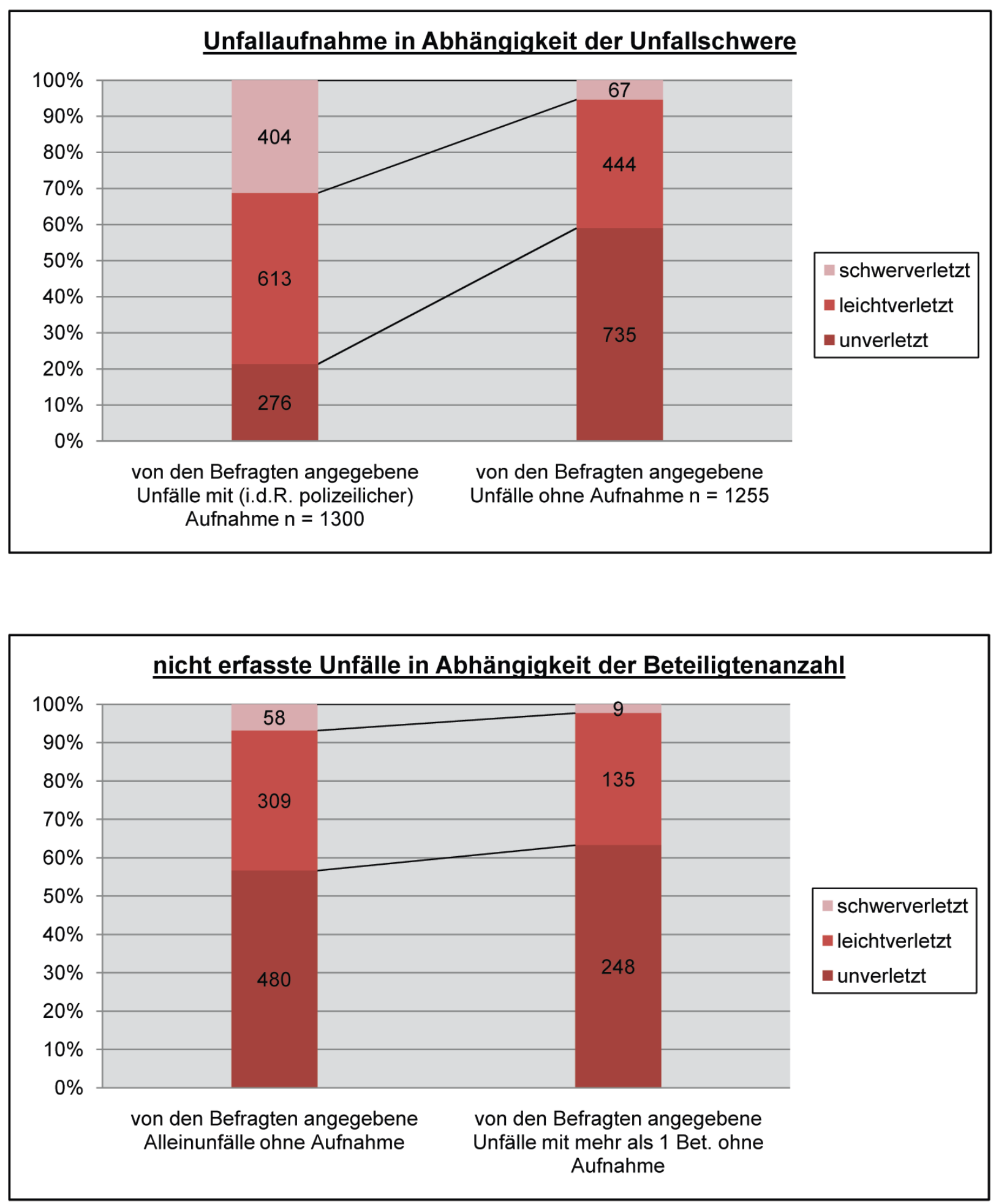


\section{GDV}

DIE DEUTSCHEN VERSICHERER

\section{Unfallforschung der Versicherer}

Wilhelmstraße 43 / 43 G, 10117 Berlin

E-Mail: unfallforschung@gdv.de

Internet: www.unfallforschung-der-versicherer.de 